\title{
WestVirginiaUniversity
}

THE RESEARCH REPOSITORY @ WVU

Graduate Theses, Dissertations, and Problem Reports

2017

\section{College Students' Sleep Quality}

Yahya Ahmed Alamir

Follow this and additional works at: https://researchrepository.wvu.edu/etd

\section{Recommended Citation}

Alamir, Yahya Ahmed, "College Students' Sleep Quality" (2017). Graduate Theses, Dissertations, and Problem Reports. 5047.

https://researchrepository.wvu.edu/etd/5047

This Dissertation is protected by copyright and/or related rights. It has been brought to you by the The Research Repository @ WVU with permission from the rights-holder(s). You are free to use this Dissertation in any way that is permitted by the copyright and related rights legislation that applies to your use. For other uses you must obtain permission from the rights-holder(s) directly, unless additional rights are indicated by a Creative Commons license in the record and/ or on the work itself. This Dissertation has been accepted for inclusion in WVU Graduate Theses, Dissertations, and Problem Reports collection by an authorized administrator of The Research Repository @ WVU.

For more information, please contact researchrepository@mail.wvu.edu. 


\title{
College Students' Sleep Quality
}

\author{
Yahya Ahmed Alamir, BSN, MAP, PhD \\ Dissertation submitted to the \\ School of Public Health \\ at West Virginia University \\ in partial fulfillment of the requirements for the degree of
}

\author{
Doctor of Philosophy \\ in \\ Public Health/ Social and Behavioral Sciences
}

Keith J. Zullig, M.S.P.H., Ph.D., Chair

Sijin Wen, PhD

Hawley Montgomery-Downs, PhD

Alfgeir L. Kristjansson, $\mathrm{PhD}$

Ranjita Misra, PhD, CHES, FASHA

Social and Behavioral Sciences Department

Morgantown, West Virginia, 2017

Keywords: Sleep quality, College students, NMUPD, BMI, HBM, TPB

Copyright 2017 Yahya Alamir 


\title{
Abstract \\ College Students' Sleep Quality
}

\author{
Yahya Ahmed Alamir, MAP
}

Poor sleep quality among college students increases the risk for lower grade point averages, compromised learning, impaired mood, and motor vehicle accidents; and associated with several unhealthy behaviors and outcomes including substances /drugs use (alcohol and medications), and weight gain. Therefore, we assessed college sleep quality in relation to the NMUPD and BMI among a large sample of college students using the fall 2010-spring 2011 national survey data (American College Health Association - National College Health Assessment (ACHA-NCHA)). In addition, we used theoretical model integrating HBM perception constructs and behavioral intention to describe and predict college students' sleep quality among a sample of WVU undergraduate students. The goal of this dissertation was to better understand students' sleep quality. Study results can help in establishing theoretically driven interventions to promote students' sleep quality.

Poor sleep was common among a large national sample of college students and among WVU college students. The NMUPD was significantly associated with poor sleep quality, and poor sleep quality significantly increases the predicted values of BMI and increases the risk of being overweight and obese. In addition, poor sleep quality was also associated with having more unhealthy days among WVU students. The HBM and the behavioral intention (TPB) have significant roles in prediction poor sleep quality among a sample of WVU college students. Our results extend the current knowledge regarding the association between NMUPD and poor sleep quality, the association between poor sleep quality and overweight and/or obesity, the association between poor sleep quality and poor HRQOL, and the significant roles of HBM and behavioral intention in explaining sleep quality among college students. Moreover, we add to the current literature the influence of the HBM on behavioral intention, which make a very significant combination in predicting sleep quality among college students. Therefore, assessment of college students' sleep quality should include screening for NMUPD, overweight and obesity, HRQOL as potential risk factors for poor sleep, and may all be interrelated. 


\section{Acknowledgement}

This project would not have been possible without the love, support, and encouragement I received from my mother, wife, kids, family, and friends. A great and special thank I must send to my mother (Mrs. Marium Alamir) who always prays for me for success. My wife (Mrs. Ebtehal Alamir) and my kids (Master. Ayham, Master. Ayman, and my little girl Maria) have great contributions to my success in producing this work. Always I realize how much they sacrificed so that I could have time away from them to do my work. I really, do not have words to adequately describe my deep gratitude for all they have provided me, though I hope to show them more achievements to come. I am not also forgetting my brothers and sister, my brothers in law, who always standing by me given all kinds of support. Finally, I would like also to thank my friend Mr. Riyadh Alhazmi, who always encourage me to keep the hard work. Thank you all for everything I have got from you that keep me on the hard work every day. 
Table of Contents

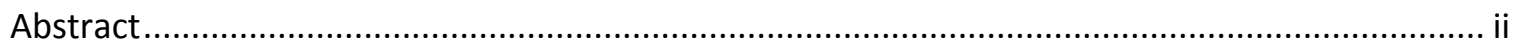

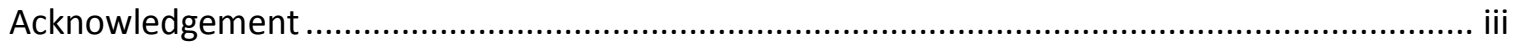

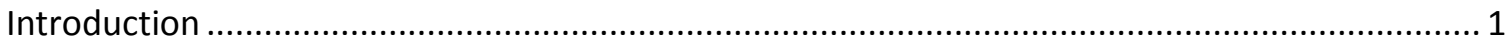

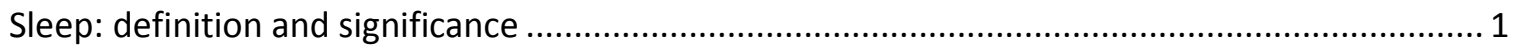

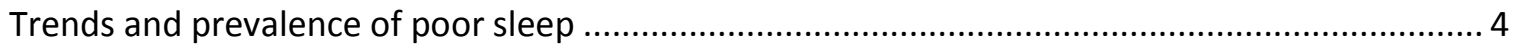

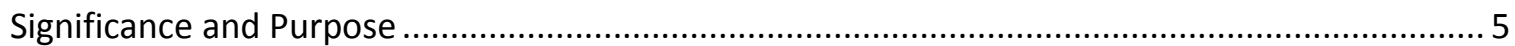

The Relationship between the Nonmedical Use of Prescription Drugs (NMUPD) and Sleep Quality

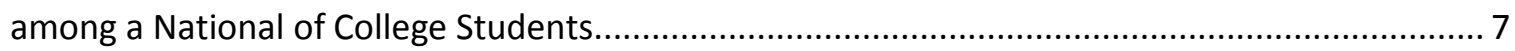

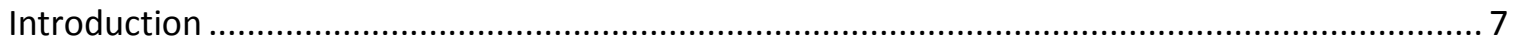

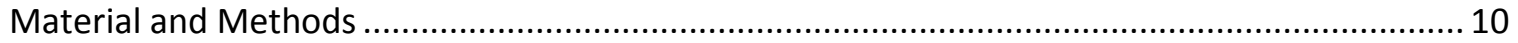

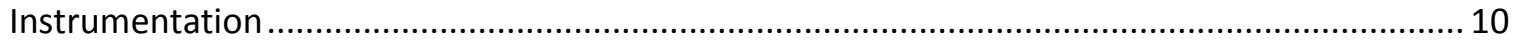

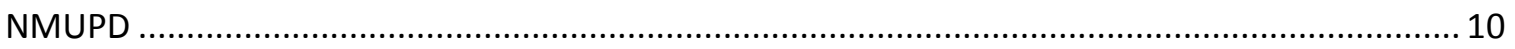

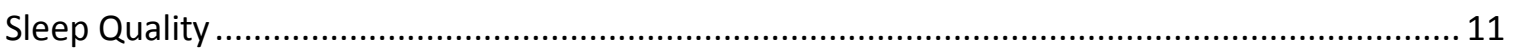

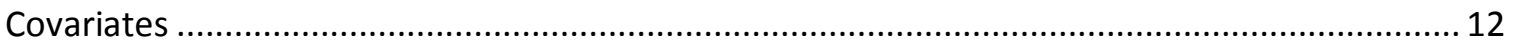

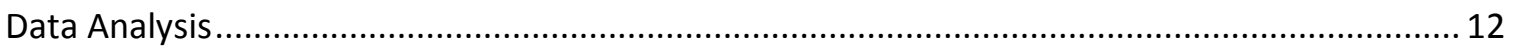

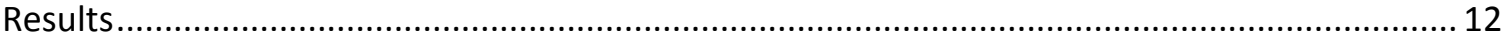

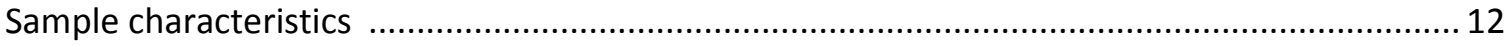

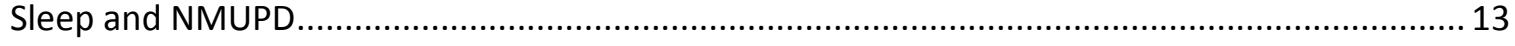

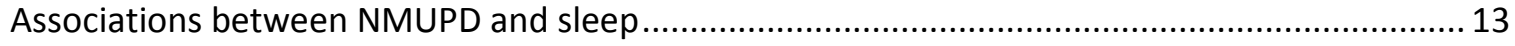

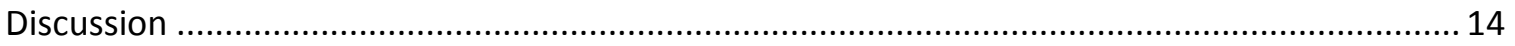

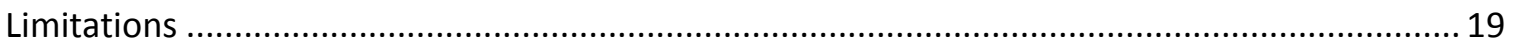

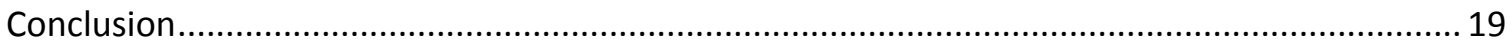

The Relationship between Sleep Quality and Body Mass Index (BMI) Measures among a National

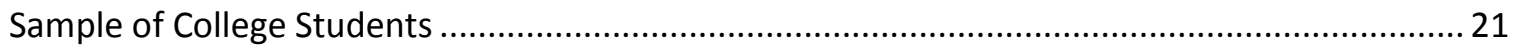

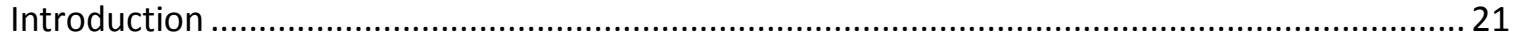

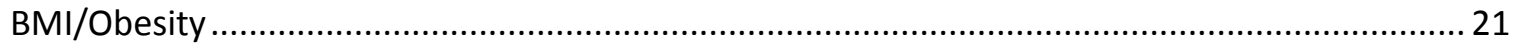

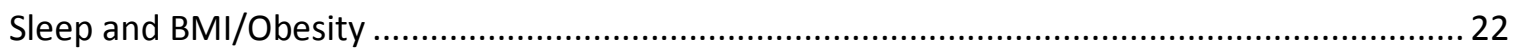

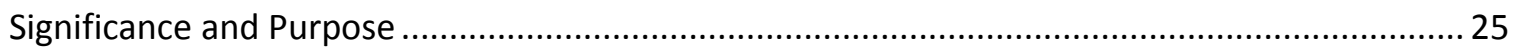

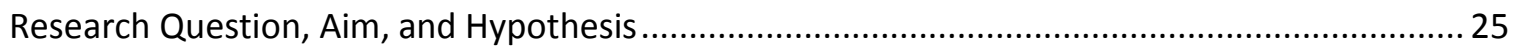

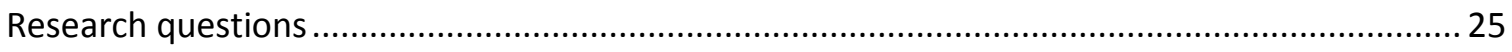

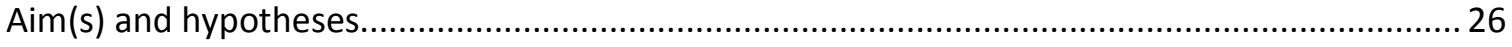




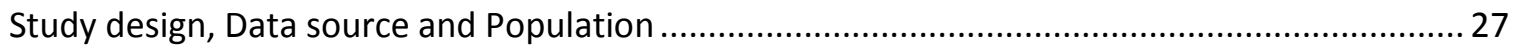

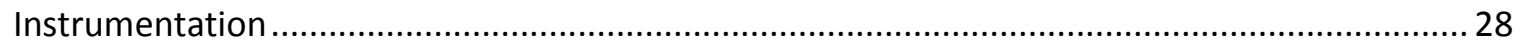

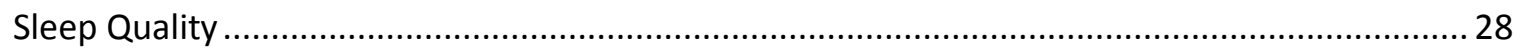

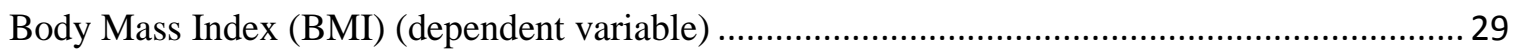

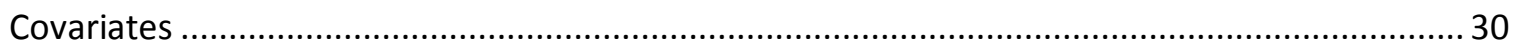

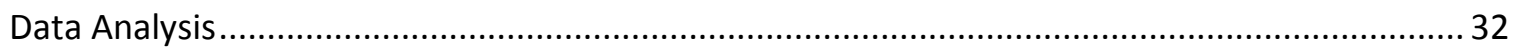

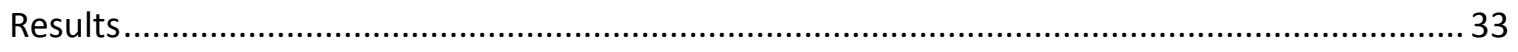

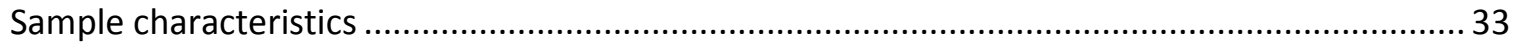

Sleep Quality and the Body Mass Index (BMI) description........................................................ 34

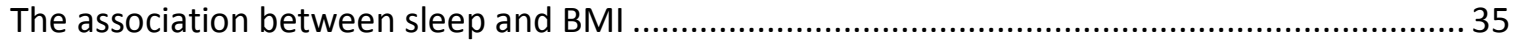

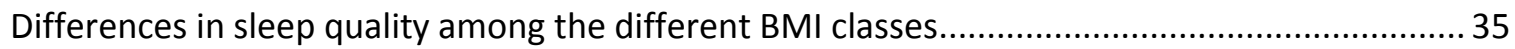

The association between the different sleep quality aspects and BMI measures (multivariate ordinal regression and Linear Regression analyses) ................................................................. 37

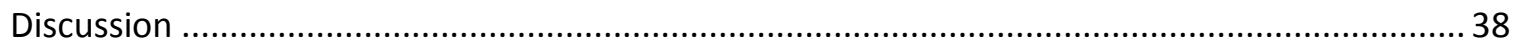

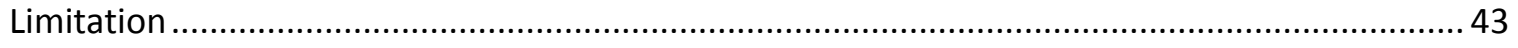

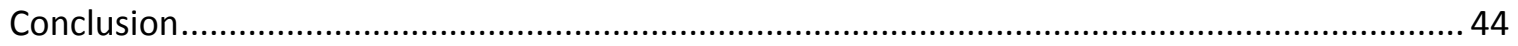

A Theory-based Description of Sleep Quality and Its Impact on Health-Related Quality Of Life Using Health Belief Model and Behavioral Intention among a Sample of WVU Undergraduates

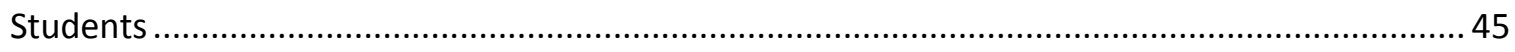

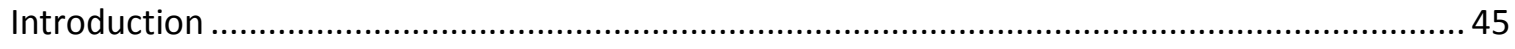

Health Belief Model and Theory of Planned Behavior ............................................................... 45

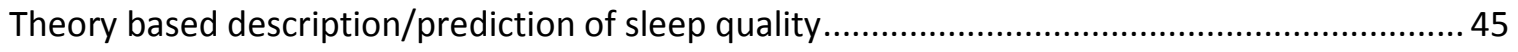

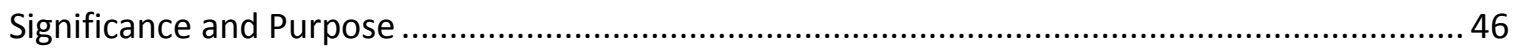

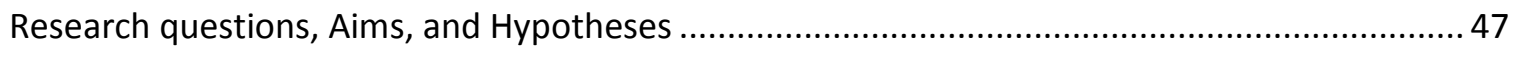

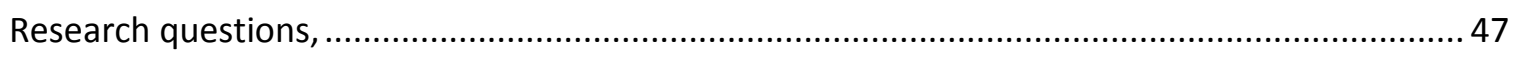

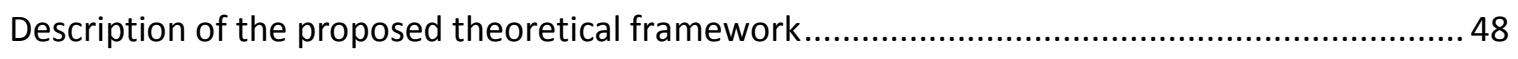

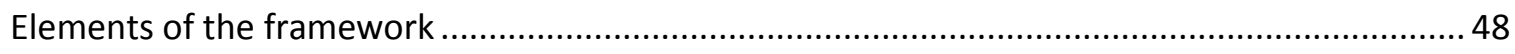

Conceptual and operational definitions of the Model's elements:............................................ 49

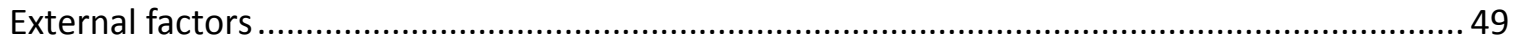

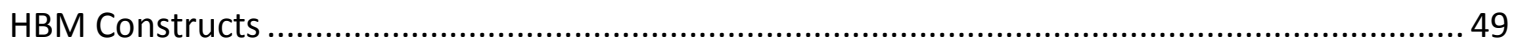

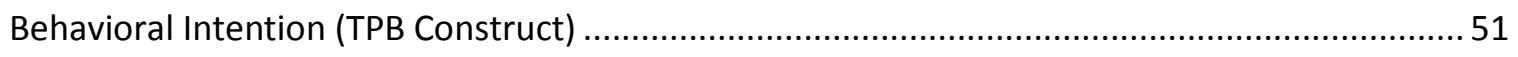

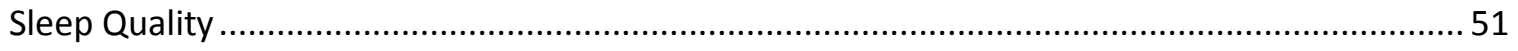

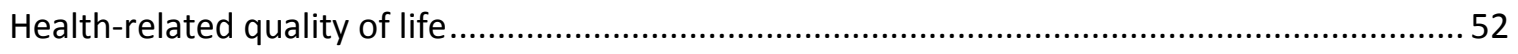


Model assumptions.

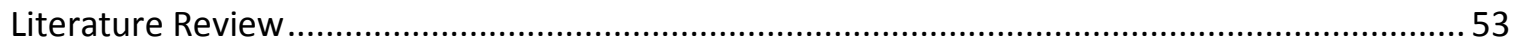

Model's external factors and sleep (first research question) ................................................... 53

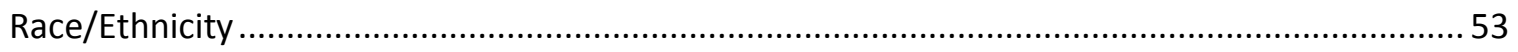

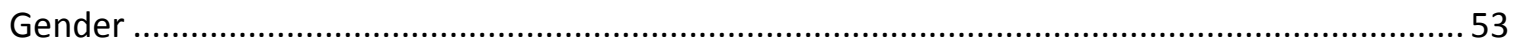

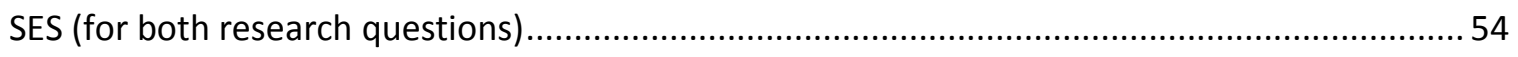

Other key important covariates (second research question) .................................................... 55

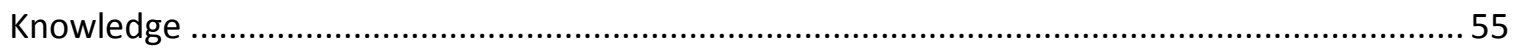

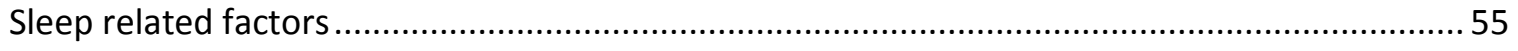

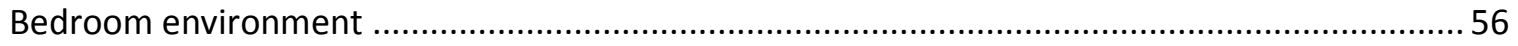

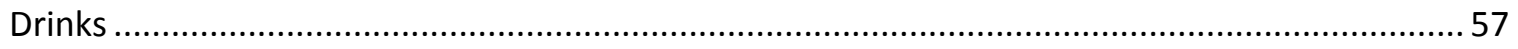

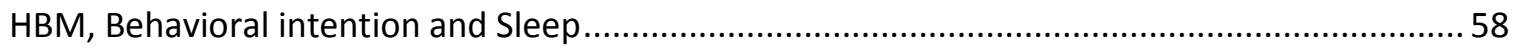

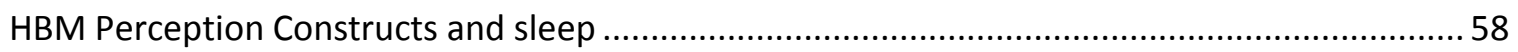

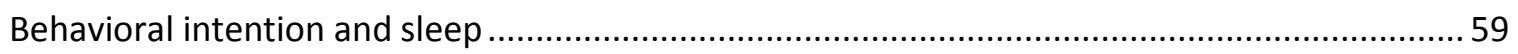

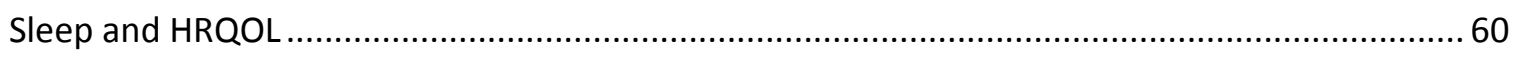

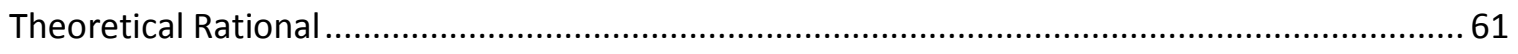

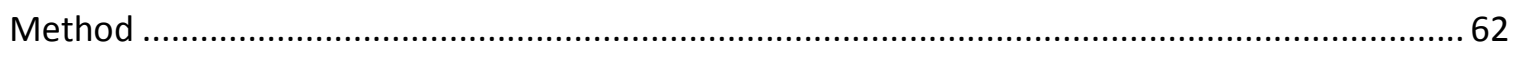

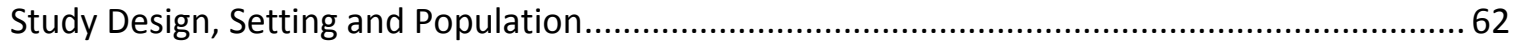

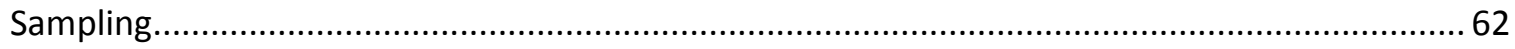

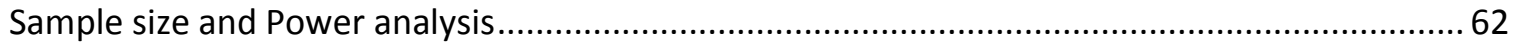

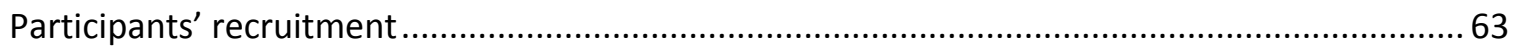

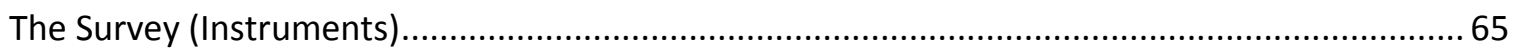

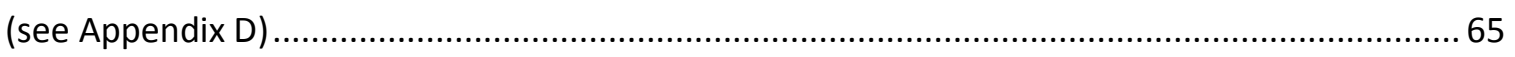

Health related quality of life: HRQOL-14 "Healthy Days Measure" ............................................. 65

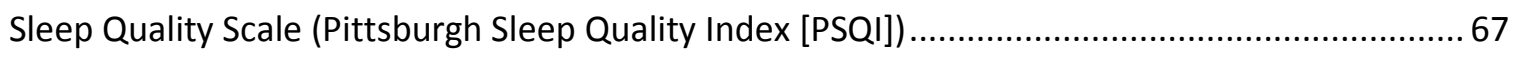

The Theory of Planned Behavior Instrument (Behavioral Intention) ...........................................68

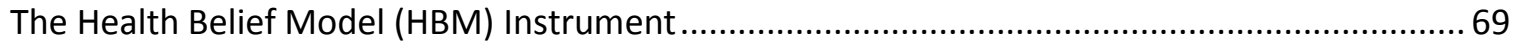

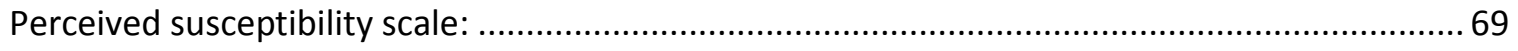

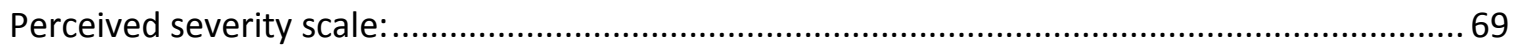

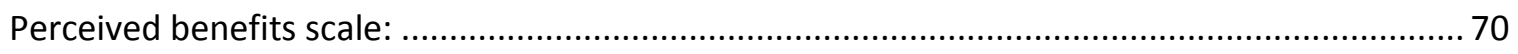

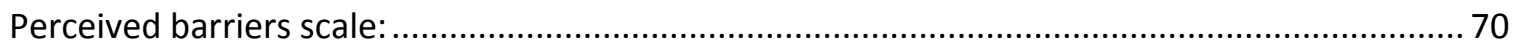

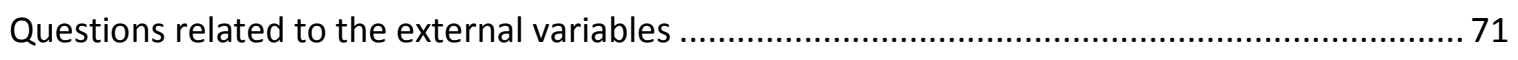

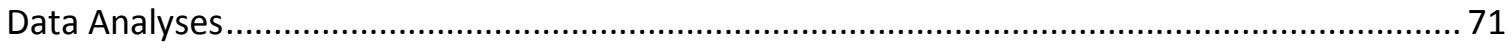




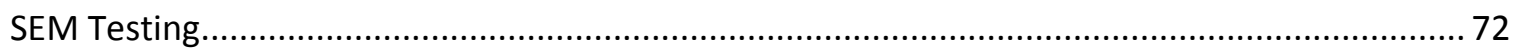

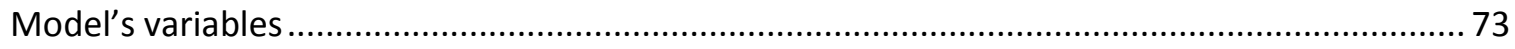

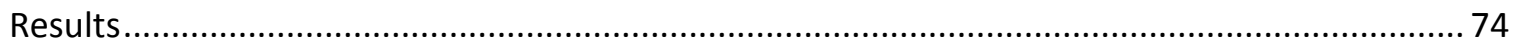

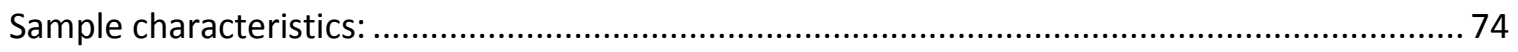

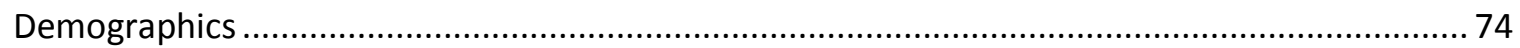

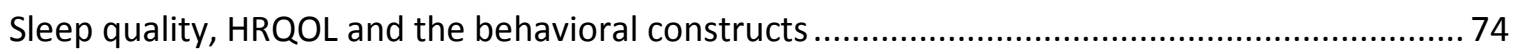

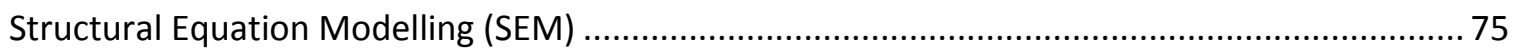

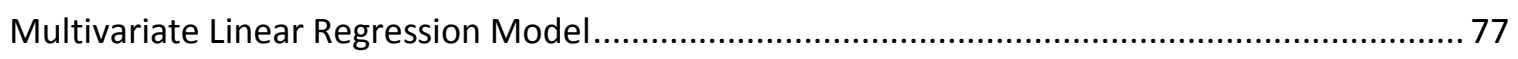

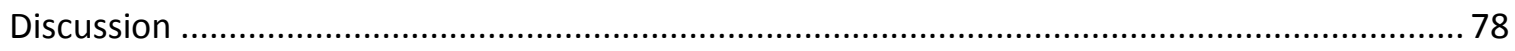

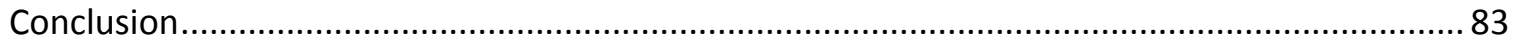

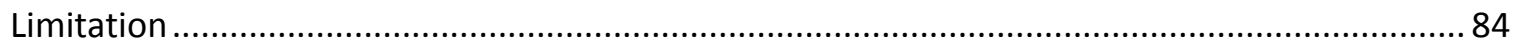

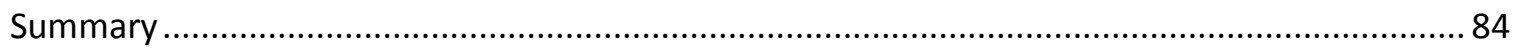

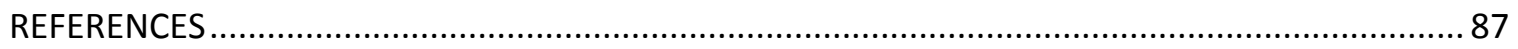

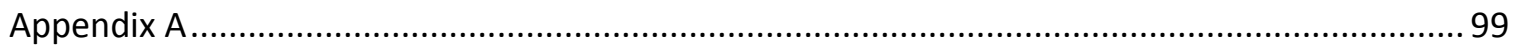

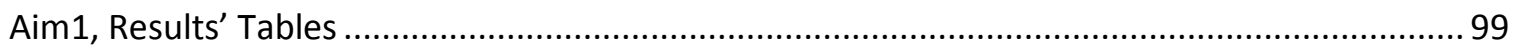

Table 1. Sample Characteristics $(\mathrm{F}=$ Frequency, $\%=$ percentage) ......................................... 100

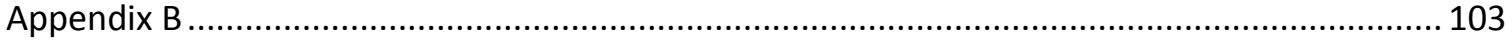

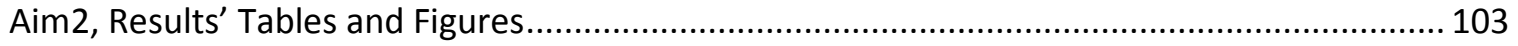

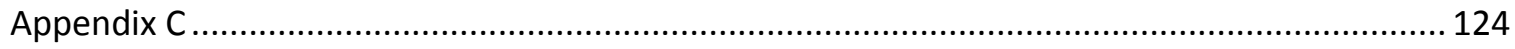

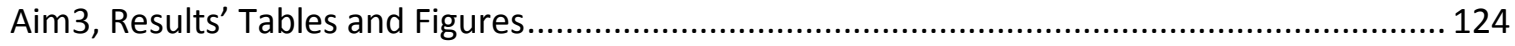

133

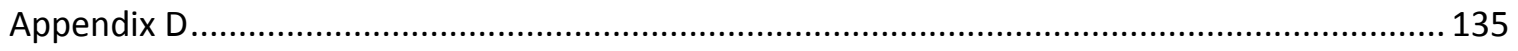

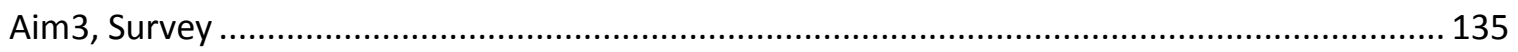

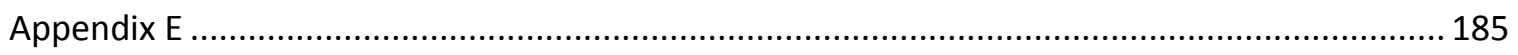

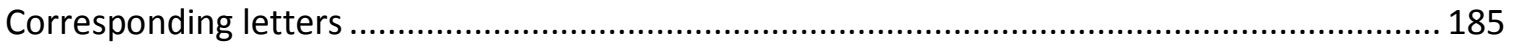

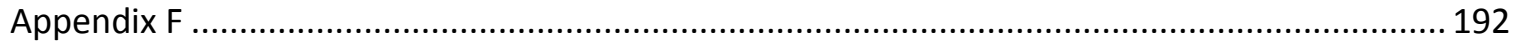

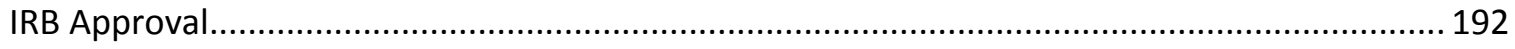


Introduction

\section{Sleep: definition and significance}

Sleep is described as a complex combination of behavioral and physiological processes (Kryger, Roth, \& Dement, 2011). Commonly, sleep is defined as a behavioral state characterized by reduced responsiveness to stimuli, decreased awareness and interaction with the environment, muscle relaxation, and reduced movement (Benca, 2012; Kryger et al., 2011). The physiological definition of sleep refers to a period of intense physiological and neurological activities including changes in the brain activities during the different stages of sleep (McLaughlin Crabtree \& Williams, 2009). A typical night's sleep (e.g. 7-8 hours of sleep duration among adults), passes through an average of four to five recurring cycles; each cycle comprises four stages of sleep. The average length of the first cycle is approximately 70 to 100 minutes, which then increases to an average of 90-110 minutes for the second and later cycles (Benca, 2012; Kryger et al., 2011). Stages of sleep are divided into non-rapid eye movement (NREM), and rapid eye movement (REM) (American Sleep Association [ASF], 2015; Benca, 2012; Kryger et al., 2011; National Institute of Neurological Disorders and Stroke [NINDS], 2014; National Sleep Foundation [NSF], 2015a; Robotham, Chakkalackal, \& Cyhlarova, 2011).

NREM sleep consists of approximately $75 \%-80 \%$ of the total sleep duration and comprises three stages: NREM 1 or stage 1, NREM 2 or stage 2 and NREM 3 or stage 3 (ASA, 2015; Benca, 2012; Kryger et al., 2011; NINDS, 2014; NSF, 2015a; Robotham et al., 2011). In NREM Stage 1, individuals progress through drowsiness and then light sleep. This stage represents an essential bridge between wakefulness and sleep; where eye movement, muscle activity, and breathing all slow down. In general, sleep in this stage can be easily disrupted; and individuals can be easily awakened from sleep (ASA, 2015; Benca, 2012; Kryger et al., 2011; 
NINDS, 2014; NSF, 2015a; Robotham et al., 2011). In NREM stage 2, eye movement stops, muscle activity decreases, breathing is regular and slow, and body temperature drops. In this stage, individuals become disengaged from the outside world; they may still hear sounds, but are unable to understand them. Then, individuals move into deep sleep during NREM stage 3 (it is also called slow-wave sleep (SWS)). In this stage, there is no eye movement or muscle activity, and breathing becomes slower. It is difficult to wake in this stage, and individuals become less responsive to, and aware of, sounds. In fact, tissue growth and repair occurs, along with energy restoration in this stage. Dreams can occur, but not as common as random eye movement (REM) stage (ASA, 2015; Benca, 2012; Kryger et al., 2011; NINDS, 2014; NSF, 2015a; Robotham et al., 2011). Finally, REM is the last stage in the sleep cycle, which describes an active stage of the brain and accounts for around $20 \%$ to $25 \%$ of total sleep duration. In this stage, individuals' muscle will be completely paralyzed and unresponsive; and more rapid and irregular breathing occurred. The majority of dreams occur in this stage (ASA, 2015; Benca, 2012; Kryger et al., 2011; NINDS, 2014; NSF, 2015a; Robotham et al., 2011). Each sleep stage provides a distinct, significant physiological and neurological function for the health of the body and mind, making it crucial to progress through all sleep stages many times during a single night sleep.

Sleep duration (i.e. the number of sleeping hours per night) is also significant for a good night sleep and important for ensuring going through sleep stages in a repeated cycle. The needed number of sleeping hours per night to complete the typical sleep cycles of sleep stages varies by age group. For example, newborns should have between 16 to 18 hours a day, preschool-aged children need between 11 to 12 hours, school-aged children should have at least 10 hours, teens need between 9 to 10 hours, and adults (including elderly) are recommended to get between 7 to 8 hours of sleep per day (Centers for Disease Control and Prevention [CDC], 
2013; National Institute of Health [NIH], 2012a). Consequently, a good night's sleep encompasses both adequate number of sleeping hours and the quality of sleep, as assessed by moving through the stages of sleep. Both sleep duration and sleep quality are significant in describing poor or good (healthy) sleep (Robotham et al., 2011). Seep quality will be used in this document as an overall outcome of sleep behavior that involves both sleep duration and aspects of sleep quality; accordingly, poor sleep quality will be used to describe the concept of short sleep duration with poor aspects of sleep quality, while good, optimal and healthy sleep will be used to describe the concept of having the recommended sleeping hours with good sleep quality aspects.

Sleep is necessary to fight off infection, support the metabolism of sugar, perform well in school, and work effectively and safely (Healthy People, 2015; Robotham et al., 2011). Getting good (healthy) sleep and treating sleep disorders improves health, productivity, wellness, quality of life, and safety on roads and in the workplace (Healthy People, 2015). Conversely, poor sleep can cause sleep disorders such as sleep apnea, insomnia, narcolepsy, and restless legs syndrome (Ram, Seirawan, Kumar, \& Clark, 2010; Wells \& Vaughn, 2012). In addition, poor sleep has been associated with a host of negative behavioral and health outcomes including substance use (e.g., tobacco and alcohol usage), physical inactivity, violent behavior, mental disturbances, suicide attempts, overweight or obesity, heart disease, drowsy driving, and risk for accidents and injuries (Benca, 2012; Fredriksen, Rhodes, Reddy, \& Way, 2004; McKnight-Eily et al., 2011; National Institute of Diabetes and Diagestive and Kidney Diseases [NIDDK], 2012). Poor sleep has also been associated with poor school performance and lower average school grades (Fredriksen et al., 2004; Ming et al., 2011). Collectively, poor sleep and sleep disorders 
reduce quality of life and productivity, increase the use of health-care services, and can cause injuries, illness, or deaths (CDC, 2008).

\section{Trends and prevalence of poor sleep}

Approximately one-quarter of the U.S. population does not get enough sleep and roughly one in three adults gets less than seven hours of sleep per night (CDC, 2015; NIH, 2013). In addition, nearly 50 to 70 million Americans chronically suffer from sleep and wakefulness disorders (long-term effect of sleep deprivation and sleep disorders) (CDC, 2015; Ram et al., 2010; Wells \& Vaughn, 2012). Among adolescents (ages 13-18 years), approximately 70\% get less than 7 hours on average school nights (Chen, Wang, \& Jeng, 2006; Eaton et al., 2010; Lauderdale et al., 2009; McKnight-Eily et al., 2011; Ming et al., 2011). Moreover, sleep disorders during adolescence may predict ongoing sleep-related problems in adulthood; such as mood disorders, heart disease, and diabetes (Dregan \& Armstrong, 2010; Fredriksen et al., 2004; Lund, Reider, Whiting, \& Prichard, 2010; Ming et al., 2011).

Indeed, poor sleep is highly prevalent health issue among college students in the United States (Tsai \& Li, 2004), with more than 60\% considered poor sleepers (Lund et al., 2010). The most common and prevalent sleep disorders among college students include: sleep apnea, insomnia, narcolepsy, and restless legs syndrome (Fredriksen et al., 2004; Ming et al., 2011; Ram et al., 2010; Wells \& Vaughn, 2012). College students are particularly susceptible to poor sleep quality due to several factors such as maturational changes. In addition, poor sleep in this vulnerable population obstructs higher cognitive functions such as learning, problem solving, and creativity, which increases their risk for lower grade point averages, compromised learning, impaired mood, and motor vehicle accidents (Hershner \& Chervin, 2014; Lund, et al., 2010). Furthermore, poor sleep among college students also appear to bring about other unhealthy behaviors such as use of alcohol and medications to induce sleep, and stimulants to compensate 
sleep deprivation. Moreover, poor sleep has been connected to weight gain, and college years appear to be an important period for increases in overweight status (Curcio, Ferrara, \& De, 2006; Lund, et al., 2010 Vela-Bueno, Fernandez-Mendoza, \& Olavarrieta- Bernardino, 2009).

\section{Significance and Purpose}

Sleep health is one of the newest objectives of Healthy People 2020. The goal for sleep health is to increase public knowledge of how adequate sleep and treatment of sleep disorders improve health, productivity, wellness, quality of life, and safety on roads and in the workplace (Healthy People, 2015). Four different objectives have been established for sleep health (Healthy People, 2015):

1. Increase the proportion of persons with symptoms of obstructive sleep apnea who seek medical evaluation.

2. Reduce the rate of vehicular crashes per 100 million miles traveled that are due to drowsy driving.

3. Increase the proportion of students in grades 9 through 12 who get sufficient sleep.

4. Increase the proportion of adults who get sufficient sleep.

This project intends to contribute in achieving the objective of increasing the proportion of adults (18+ years old) who get sufficient sleep (defined as 8 or more hours for those aged 18 to 21 years and 7 or more hours for those aged 22 years and older, on average, during a 24-hour period) (Healthy People, 2015). It is crucial to study and describe sleep quality among college students; since poor sleep among this vulnerable population increases their risk for several unhealthy behaviors (drugs/substances and medication use), and obstruct their academic achievements. Such research may help in attaining better understanding of students' sleep 
quality, in order to increase the proportion of students who get sufficient sleep, and to prevent sleep-related disorders and/or negative health-related outcomes.

This dissertation aims at examining students' sleep quality in relation to the nonmedical use of prescription drugs (NMUPD) (Aim \#1) and Body Mass Index (BMI) (Aim \#2) among a large sample of college students. Aim \# 3 attempts to describe sleep quality of West Virginia University (WVU) college students using the Health Belief Model (HBM) and behavioral intention form the Theory of Planned Behavior (TPB). The three aims utilized cross-sectional designs. Aim \#1 and Aim \#2 analyses was based on secondary data, using the fall 2010-spring 2011 American College Health Association - National College Health Assessment (ACHANCHA). Aim \#3 was based on primary data collection obtained from a sample of undergraduate students attending WVU. 
The Relationship between the Nonmedical Use of Prescription Drugs (NMUPD) and Sleep Quality among a National of College Students.

\section{Introduction}

Prescription medications designed to treat pain, anxiety, sleep disorders and other medical problems have proliferated in the U.S. However, their increased availability has also lead to increased nonmedical use (NMU). Nonmedical use of prescription drugs (NMUPD) refers to the use of medication for reasons, or in ways, or in amounts, not intended by a doctor; taken without a prescription; or taken by someone other than the person for whom they are prescribed (National Institute on Drug Abuse [NIDA], 2015; Substance Abuse and Mental Health Services Administration [SAMHSA], 2015).

There were 15 million indiviuals, aged 12 years or older, who engaged in NMUPD, and NMUPD is more common than use of any other drug, with the exception of marijuana (Hughes, Williams, Lipari, Bose, Copello, \& Kroutil, 2016; SAMHSA,2015). Lifetime prevalence of NMUPD is $20 \%$ among college students for at least one prescription medication class (i.e. sedatives, opioids, or stimulants) (McCabe, West, Teter, \& Boyd, 2014). In addition, college students also have the highest NMU of opioid painkillers (McCabe \& Teter, 2007). NMU of antidepressants is unexplored in many large-scale epidemiological surveys. However, $8 \%$ of Americans 12 years or older used antidepressants between 2005 and 2008 for reasons other than depression (Pratt, Brody, \& Gu, 2011). Such usage increased from $2.4 \%$ to $10.8 \%$ between 2007 and 2010 among adults (National Center for Health Statistics [NCHS], 2014).

The most commonly reported reasons for NMUPD among college students are to "stay awake to study" and/or to "increase concentration" (Clegg-Kraynok, McBean, \& MontgomeryDowns, 2011; McCabe \& Teter, 2007). However, the association between NMUPD and sleep 
quality among college students has not been generally explored, with the exception of stimulant use. Clegg-Kraynok (2010) found that college students not only have poorer sleep quality and shorter sleep duration than adolescents but also are more likely to use psychostimulants nonmedically (defined as stimulants used for the treatment of Attention-Deficit/Hyperactivity Disorder $[\mathrm{ADHD}])$ to compensate for shortened sleep; students with lifetime NMU of psychostimulants report poorer sleep quality and more sleep disturbance than nonusers (CleggKraynok, et al., 2011).

However, the association between sedative, painkiller, and antidepressant use and sleep quality has not been explored among college students, and only sparsely in the general population. Sedatives are often referred to as sleep aids (Chong, Fryar, \& Gu, 2013). Chong et al. (2013) reported that noninstitutionalized U.S. adults who obtain either fewer than five hours or more than nine hours of sleep, use sleep aids in higher proportions (6.0\% and $5.3 \%$ respectively). Use of sleep aids is also high among adults who report sleep-related disorders and trouble sleeping (Chong et al., 2013).

Painkillers can significantly disrupt sleep architecture in healthy adults (Dimsdale, Norman, DeJardin, \& Wallace, 2007). Dimsdale et al. (2007) found both sustained-release morphine sulfate and methadone use significantly reduced deep sleep and increased stage N2, resulting in overall sleep quality disturbance. Studies of adolescents' use of pain relievers showed they have more trouble sleeping (in the past six months) than nonusers (McCabe, West, and Boyd, 2013). Poor sleep quality was also reported by $80.6 \%$ of prescription opioid users compared to healthy controls (Hartwell, Pfeifer, McCauley, Moran-Santa, \& Back, 2014). The prescription opioid-dependent group also had shorter sleep duration, longer sleep latency, and more awakening times. 
Antidepressants, on the other hand, change and improve sleep architecture, specifically by decreasing the amount of REM sleep and increasing its onset latency in both healthy individuals and depressed patients (Lam, 2005; Wilson \& Argyropoulos, 2005). These effects vary by drugs, with some short-term sleep disturbance early in treatment (e.g., clomipramine and the selective serotonin receptor inhibitors [SSRIs]), while others are sleep-promoting (e.g., amitriptyline and the newer serotonin 5-HT2-receptor antagonists) (Wilson \& Argyropoulos, 2005). However, some antidepressants drugs may cause insomnia and daytime sleepiness (Lam, 2005; Mayers \& Baldwin, 2005). In general, sleep improved with effective antidepressant treatment within 3-4 weeks (Wilson \& Argyropoulos, 2005).

The current literature on NMUPD and sleep quality among both college students and in the general population are limited because: (1) many large-scale epidemiological surveys of prescription drug use have not included NMU of antidepressants; and (2) they have not explored important demographic and behavioral covariates, including sleep disorders and other gateway drugs (NMU of stimulants, in particular, is associated with increased use of other drugs including alcohol, cocaine, cigarettes, and marijuana) (Hershner \& Chervin, 2014; McCabe \& Teter, 2007).

Therefore, our study purpose was to use a large, national college student sample to examine the associations between four classes of NMUPD (antidepressant, painkillers, stimulants, and sedatives) in the past 12 months and five aspects of sleep quality (Enough Sleep, Early Awakening, Daytime Sleepiness, Difficulty Falling Asleep, and Problem associated with Daytime Sleepiness) in the past seven days. Specifically, we sought to take into consideration known limitations, and to include important demographic and behavioral covariates that have not been controlled in previous studies. Given the relatively exploratory nature of the study, no specific hypotheses were developed. However, in line with the extant literature, we expect a 
positive association between NMUPD and sleep quality as reflected by five aspects of sleep behavior. We examined potential associations that may exist with any NMUPD, as well as within each drug class individually.

\section{Material and Methods}

This study utilized data from the fall 2010 and spring 2011 National American College Health Association-National College Health Assessment (ACHA-NCHA). We were granted access to these data through a formal request to the American College Health Association. There were 42 and 129 institutions that participated in the fall $2010(n=30,263)$ and spring 2011 $(\mathrm{n}=116,254)$ NCHA, respectively. However, we selected only those schools (39 and 129 during fall-2010 and spring-2011, respectively) that surveyed all available students or used a random sampling technique ( $\mathrm{N}=135,874$; ACHA, 2011). The overall response rates were $30.9 \%$ and 28.5\% for fall 2010 and spring 2011, respectively. Missing values (missing at random), $n=2,663$ and 16,321 individuals, were excluded from descriptive analysis and from inferential analysis, respectively.

\section{Instrumentation}

\section{NMUPD}

The independent variables included four classes of nonmedical prescription drugs: antidepressants, painkillers, sedatives, and stimulants. The four questions used to assess NMUPD were: "Within the last 12 months, have you taken any of the following prescription drugs that were not prescribed to you: (1) [Antidepressants (e.g., Celexa, Lexapro, Prozac, Wellbutrin, Zoloft)]; (2) [Pain killers (e.g., OxyContin, Vicodin, Codeine)]; (3) [Sedatives (e.g., Xanaz, Valium)]; (4) [Stimulants (e.g., Ritalin, Adderall)]?" Response options for each question were 1) yes or 2) no. 


\section{Sleep Quality}

The dependent variables measured aspects of sleep quality. Specifically, we assessed

sleep quality in the past seven days, as reflected by participants' experiences with: Enough Sleep, Early Awakening, Daytime Sleepiness (frequency), Difficulty Falling Asleep, and Problem with Daytime Sleepiness (magnitude). Two aspects of Daytime Sleepiness have been assessed: the frequency of feeling sleepy during the day, and the magnitude of feeling sleepy (problem associated with sleepiness during the day). The following questions were chosen to match the study aim: 1) Enough Sleep = "On how many of the past 7 days did you get enough sleep so that you felt rested when you woke up in the morning?"; 2) Early Awakening = "In the past 7 days, how often have you awaken too early in the morning and couldn't get back to sleep?"; 3) Daytime Sleepiness = "In the past 7 days, how often have you felt tired, dragged out, or sleepy during the day?"; 4) Difficulty Falling Asleep = "In the past 7 days, how often have you had an extremely hard time falling asleep?"; 5) Problem with Daytime Sleepiness = asked "In the past 7 days, how much of a problem have you had with sleepiness during your daytime activities?" Response options for the first four questions ranged from zero to seven days; response options for question five was: $1=$ no problem at all, $2=$ a little problem, $3=$ more than a little problem, $4=$ a big problem, and $5=$ a very big problem.

For analysis purposes, particularly descriptive and Chi-square tests, we grouped each sleep quality measure into three groups based on the literature (Becker, Adams, Orr, \& Quilter, 2008): Enough Sleep (6-7 days = optimal, $2-5$ days $=$ middle, and 0-1= poor). Early Awakening, Daytime Sleepiness, and Difficulty Falling Asleep were coded as: (6-7 days = poor, 2-5 days =

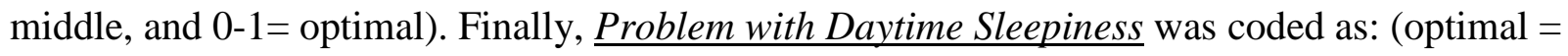
having no problem with sleepiness, poor $=$ combining having a little, more than a little, a big, and a very big problem with sleepiness during the daytime activities). 


\section{Covariates}

Study covariates (Table 1) included gender, race, age, body mass index (BMI) (obtained from the reported height and weight), other substance use (i.e. cigarette, alcohol, and marijuana use in the past 30 days), diagnosed medical disorders (depression, insomnia, ADHD, sleeprelated disorder), and overall perceived stress (participants were asked to rate their overall level of stress they have experienced in the past 12 months, on a five-point Likert scale ranges from no stress to tremendous stress). These covariates were included because of known associations with sleep quality, NMUPD, and to control for any interrelated effects between the proposed covariates, NMUPD and poor sleep (Hershner \& Chervin, 2014; McCabe \& Teter, 2007; Tsai \& Li, 2004).

\section{Data Analysis}

Descriptive statistics were followed by inferential ordinal regression models to examine the associations between any NMUPD (antidepressants, painkillers, sedatives, and stimulants) in the past 12 months, and sleep quality in the past seven days (Enough Sleep, Early Awakening, Daytime Sleepiness, Difficulty Falling Asleep, and Problem with Daytime Sleepiness) as ordinal variables; while controlling for the study covariates using SAS 9.4. We excluded gender from the covariates when modelling based on gender.

\section{Results}

\section{Sample characteristics $(N=135,874)$}

Sample characteristics are displayed in Table 1 (Appendix A). The majority of participants were female (65.3\%), and Non-Hispanic white $(68.1 \%)$. Around $66 \%$ had used alcohol in the past 30 days, roughly $15 \%$ reported smoking cigarettes during last 30 days and around $15.4 \%$ using marijuana during the same period. 


\section{Sleep and NMUPD}

Table 2 (Appendix A) presents participants' experiences with the five different aspects of sleep quality in the past seven days; $22 \%$ of students reported not getting Enough Sleep (0-1 day/week); $24.3 \%$ reported having 2-5 days/week of Early Awakening and could not get back to sleep; 62.3\% reported Daytime Sleepiness for 2-5 days/week; and 28.5\% had Difficulty Falling Asleep during 2-5 days/week. In addition, the majority of students (90\%) reported having Problems with Daytime Sleepiness in the past seven days.

Females $(69.6 \%)$ reported more frequently than males (30.4\%) did that they had 0-1 days of Enough Sleep; females also reported more days of Daytime Sleepiness (72.7\%) than males (27.3\%) for 6-7 days/week. In addition, having 6-7 days of Early Awakening and 6-7 days of having Difficulty Falling Asleep were higher among females (69.4\% and 69\%, respectively) than males (30.6\% and 31\%, respectively). Having Problem with Daytime Sleepiness was also higher among females $(66.6 \%)$ than males $(33.4 \%)$.

Fourteen percent of students reported NMUPD, in at least one class, in the past 12 months (Table 2). Among those who reported NMUPD, 7.5\% reported use of painkillers, $7.4 \%$ use of stimulants, $4 \%$ use of sedatives, and $3 \%$ use of antidepressants, with females reporting higher usage than males in all classes.

\section{Associations between NMUPD and sleep}

Results of the ordinal regression analysis (Odds Ratio, [95\% Confident Interval]) are in Table 3 (Appendix A). Significant associations were found between NMUPD and sleep behaviors after adjusting for covariates. Students who reported NMUPD in at least one class of prescription drugs in the past 12 months had significantly fewer days of getting Enough Sleep $(0.86[95 \% \mathrm{CI}=0.83-0.88])$, more days of Early Awakening $(1.28[95 \% \mathrm{CI}=1.24-1.33])$, Daytime Sleepiness (1.23 [1.19-1.26]), and more days of having Difficulty Falling Asleep (1.32 
[1.28-1.37]). Among students who reported NMUPD, males reported significantly more days of Early Awakening (1.33 [1.26-1.41]) than females (1.26 [1.21-1.31]), and more days of having Difficulty Falling Asleep (1.44 [1.36-1.52]), than females (1.26 [1.22-1.31]).

In general, analyses did not differ when analyzing each drug class separately, even after controlling for covariates. Students who reported NMU of painkillers reported significantly fewer days of getting Enough Sleep (0.84 [0.80-0.88]), significantly more days of Early Awakening (1.28 [1.22-1.34]), more days of Daytime Sleepiness (1.16 [1.11-1.22]), and having Difficulty Falling Asleep (1.27 [1.21-1.33]) ( $\mathrm{p}<.0001)$. Among painkiller users, males reported significantly more days of Early Awakening (1.34 [1.24-1.45]) than females (1.24 [1.17-1.31]), and more days of having Difficulty Falling Asleep ( $\mathrm{OR}=1.33[1.23-1.43]$ ), than females $(\mathrm{OR}=1.23[1.23-1.43])$

This pattern was consistent for the NMU of stimulant use with the exception that males had fewer days of getting Enough Sleep (0.88 [0.82-0.95]), having more days of Early Awakening (1.15 [1.06-1.25]), and having Difficulty Falling Asleep (1.21 [1.12-1.30]).

Finally, NMU of sedatives was significantly associated with Problem with Daytime Sleepiness (1.10 [1.03-1.17]), more days of Early Awakening (1.12 [1.05-1.19]), and having Difficulty Falling Asleep (1.17 [1.09-1.24]). Among sedatives users, only females reported significantly more days of Early Awakening and Difficulty Falling Asleep (1.14 [1.05-1.24], and $1.18[1.09-1.27]$ respectively).

\section{Discussion}

To our knowledge, this is the first study that has examined association between NMUPD (antidepressant, painkillers, sedatives, and stimulants), and sleep quality using a large, national sample of college students. These results have revealed that poor sleep is highly prevalent among 
college students across all five measured aspects of sleep quality. The majority of students (90\%) reported Problems with Daytime Sleepiness, and about 62.3\% reported Daytime Sleepiness for 2-5 days/week; this is consistent with prior research on poor quality sleep (Tsai \& $\mathrm{Li}, 2005$; Lund et al., 2010). Female students reported poorer sleep quality, and that they felt more tired, dragged out, or sleepy during the day (Daytime Sleepiness) than did males, suggesting females have poorer sleep quality than males (Tsai \& Li, 2005).

While $14 \%$ of students reported NMUPD in the past 12 months, this is less than a previous report of $20 \%$ lifetime use (McCabe et al., 2014). McCabe et al. (2009) reported that $45 \%$ of college students had used painkillers, $46 \%$ had used sedatives, and $61 \%$ had used stimulants in their lifetime. Among adults, NMUPD has increased from $2.4 \%$ to $10.8 \%$ between 2007 and 2010 (NCHS, 2014). Nevertheless, the lower prevalence of NMUPD in this study is likely due to our assessment of NMUPD in the past 12 months as opposed to lifetime use. In addition, the survey had included the first part of the NMUPD definition (inappropriate use of a legitimate prescription), the numbers here may have been much higher if the other ways of NMUPD (e.g. use of medication for reasons, or in ways, or in amounts, not intended by a doctor) were included in the questions.

Consistent with our expectations, findings confirm a significant association between NMUPD (during the past 12 months) and poor sleep quality, even after controlling for key covariates (Babcock \& Byrne, 2000; Chong et al., 2013; Clegg-Kraynok ,2010 \& 2011; Dimsadale et al., 2007; Hartwell et al., 2014; Hershner \& Chervin, 2014; McCabe et al., 2013). However, no significant association was found between antidepressants use and poor sleep, possibly due to the fact that most antidepressant drugs have few short-term effects on sleep and with longer-term use eventually promote sleep (Wilson \& Argyropoulos, 2005). The most 
consistent short-term effect of antidepressants is on REM sleep ( Lam, 2005; Wilson \& Argyropoulos, 2005). However, some classes of antidepressants may cause insomnia, and daytime sleepiness in the early stage of treatment (Lam, 2005; Mayers \& Baldwin, 2005). Therefore, the use of antidepressants (either medical use or NMU) during the past year may have beneficial effects on sleep among both depressed and non-depressed students (Wilson \& Argyropoulos, 2005). However, additional research on the association between antidepressants and sleep should assess potential risks and benefits of NMU.

In our study, any NMUPD was significantly associated with reduced days of Enough Sleep; increased days of Early Awakening, Daytime Sleepiness, and having Difficulty Falling Asleep. Hence, our findings suggest a pattern of poor sleep quality among students who engage in NMU of any prescription drug class.

Similar to NMUPD overall, NMU of stimulants significantly reduced days of Enough Sleep; increased days of Early Awakening, Daytime Sleepiness, and having Difficulty Falling Asleep; which concurs with prior studies of poorer sleep quality, shorter sleep duration, and sleep disturbances among users than nonusers (Clegg-Kraynok ,2010 \& 2011). Thus, our results support research demonstrating that stimulants increase sleep latency, decrease sleep duration, and lead to increase sleep disturbance (Babcock \& Byrne, 2000; Hershner \& Chervin, 2014). Since stimulants increase dopamine levels in the brain, the result may be a sense of euphoria, improved mental alertness, focus and concentration, increased physical endurance, reduced appetite, extended wakefulness, increased energy levels, enhanced self-esteem, and elevated mood (Caplan, Epstein, Quinn, Stevens, \& Stern, 2007; Khantzian, 1997; United States Drug Enforcement Administration [U.S. DEA], 2015). College students' use stimulants to stay awake and study because stimulants delay sleep and increase alertness and wakefulness (Babcock \& 
Byrne, 2000; Clegg-Kraynok, 2010 \& 2011; Hershner \& Cherwin, 2014). However, regular use of stimulants may disrupt brain activity where an individual's cycles between an improving and worsening mood thereby negatively impacting sleep patterns. Thus, our findings extend knowledge on the impact of stimulants on sleep patterns and sleep quality among college students.

NMU of painkillers showed a similar pattern of associations with sleep quality and duration as did stimulants and overall NMUPD. NMU of painkillers has been found to be associated with less sleep duration, longer sleep latency, increase trouble sleeping and more awake time among adults (McCabe et al., 2013; Hartwell et al., 2014). Although opioids (e.g. hydrocodone, oxycodone, morphine, and codeine) are prescribed to relieve pain as they inhibit the transmission of pain signals (NIH, 2016b), misuse and abuse is common because they produce euphoria in addition to pain relief (NIH, 2016; SAMHSA, 2016). Opioid painkillers also interrupt sleep by increasing wakefulness (which may lead to having difficulty falling asleep), increasing the number of shifts in sleep-waking states (increasing wake time), decreasing total sleep time (getting insufficient sleep), and affect sleep stages (reducing deep sleep and increasing light sleep) (Dimsdale, et al., 2007; Hartwell, et al., 2014).

The interrelated effects between chronic pain/severity, painkillers, and poor sleep quality in adults and among prescription opioid dependents are supported by previous studies (Dimsdale, et al. 2007; Hartwell, et al., 2014). Both pain severity and opioid use disrupt sleep (Hartwell, et al., 2014); however, opioid use disturbs sleep even in pain-free individuals (Dimsdale, et al. 2007). Although our cross-sectional research design precludes temporal conclusions regarding the role of opioid use in sleep disruption, our analyses did control for sleep-related disorders. 
Findings highlight the importance of including painkiller use and pain assessments when assessing sleep quality among college students.

NMU of sedatives was significantly associated with Problem with Daytime Sleepiness; more days of Early Awakening early; and having Difficulty Falling Asleep. Although our study controlled for depression, a large body of literature suggests that individuals who engage in NMU of sedatives also suffer from depression (Conway et al., 2006; Brunette et al., 2003). Sedatives decrease central nervous system activity (Apantaku-Olajide, 2011) to produce a drowsy or calming effect. However, risk of addiction is high, especially when used with opioids and stimulants (SAMHSA, 2009) to compliment the psychoactive (euphoric) effects (Hernandez \& Nelson, 2010).

Sedatives slow brain activity and cause a relaxing effect that is helpful for people with anxiety or sleep problems, but also cause sleepiness (NIDA, 2016). Therefore, dependence on and tolerance for NMU of sedatives for euphoric effects can lead to adverse health outcomes, including sleep disturbance (Chong et al., 2013). Interestingly, sedatives are often refereed to and used as sleep aids, and the rate of their use is high among those who suffer from short ( $<5$ hours) and long (>9hours) sleep (Chong et al., 2013). This may be done to compensate for short sleep; conversely, long sleep is non-optimal and may result from high sedatives use. Regardless, although the current study cannot be used to deduce causation, the association between NMU of sedatives and sleep supports the potential for drug use leading to poor sleep quality.

Finally, we found significant gender differences in NMUPD and sleep. Associations were generally stronger among males for NMU of stimulants and among females for NMU of sedatives. Possibly these variations can be explained by the following. Though, among the nonmedical users of stimulants there are more females $61 \%(5,881)$ than males' $(39 \%, n=3$, 
781), the physiological characteristics of females may play an important role in the effect of stimulants which may hinder the impact they may have on sleep. Among sedatives users there are more females (62\%) than males (37\%). In addition, females had poorer sleep quality as shown in our results and previous studies (Tsai \& Li, 2004), and suffer from having more days of Early Awakened and Difficulty Falling Asleep than males which has been exacerbated by sedative use.

\section{Limitations}

Results should be interpreted in the context of several study limitations. These data were cross-sectional and self-reported, and thus vulnerable to potential recall bias and socially desirability for subjective measures. The analyses were limited to questions available from this national survey, specifically the participants' sleep in the past seven days. Assessing sleep over just one week may not reflect typical sleep behavior. The ACHA did not include additional potential confounding variables such as caffeine use, which may also influence sleep patterns and behaviors (Hershner \& Chervin, 2014). Finally, since colleges self-select to participate in the ACHA survey, results cannot necessarily be considered generalizable to the larger U.S. college population.

\section{Conclusion}

Despite these limitations, our study included several strengths. Among them, the finding that poor sleep was common in this sample of college students and that these results show significantly increased odds ratios for poor sleep with NMUPD. Our study extends the current literature by revealing associations between NMUPD and sleep in terms of several specific aspects of poor sleep quality, but also among several classes of prescription drugs based on gender. We submit that assessment of both sleep quality and NMUPD behavior among college 
students should the other, potentially synergistic, risk factor in screening and treatment initiatives and research, toward better understanding and improving college students' sleep health, productivity, wellness, quality of life, and academic achievement. 


\section{The Relationship between Sleep Quality and Body Mass Index (BMI) Measures among a National Sample of College Students}

Introduction

\section{BMI/Obesity}

Body Mass Index (BMI) is a useful measure of overweight and obesity, and considered as an important and reliable indicator of obesity prevalence in large populations (Carpenter et al., 2013; NIH, 2012b). Among adults, BMI is used as a screening tool to identify possible weight problems (CDC, 2015a; NIH, 2012b). BMI is calculated from a person's weight and height. The standard weight status categories associated with BMI scores for adults are (CDC, 2015; NIDDK, 2012): underweight $(\mathrm{BMI}=<18.5)$, normal weight $(\mathrm{BMI}=18.5-24.9)$, overweight $(\mathrm{BMI}=25-29.9)$, and obese [three classes:1,2, and 3, (BMI of 30 or greater)]. Overweight and obesity refer to body weight that is greater than what is considered healthy for a certain height (NIH, 2012b). In the United States, the obesity epidemic is increasing among all age groups and remains a leading public health problem (Food Research \& Action center, 2015). Among adults, more than two-thirds are considered to be overweight or obese; and more than one-third (or 78.6 million) are obese (CDC, 2015a; NIDDK, 2012; NIH, 2012b).

Overweight and obesity increase the risk for many chronic diseases and health problems including, but not limited to, coronary heart disease, hypertension, arthritis, diabetes, stroke, certain cancers, kidney diseases, sleep apnea and respiratory problems (CDC, 2015; U.S. National Library of Medicine, 2015). Several factors that contribute to weight gain and obesity include genetics, family, environment, culture, medicine, and sleep, particularly lack of sleep. Moreover, it has been found that lack of sleep may make it harder to lose weight (CDC, 2015; NIDDK, 2012). 


\section{Sleep and BMI/Obesity}

Overweight and obesity are also related to sleep problems. Poor sleep in general is connected to overweight and obesity, as people who sleep less prefer foods that are higher in calories and carbohydrates, which can lead to overeating, weight gain, and obesity (NIDDK, 2012). In children and adolescents, poor sleep is consistently associated with higher BMI (Magee \& Hale, 2012; Marshall, Glozier, \& Grunstein, 2008; Mitchell, Rodriguez, Schmitz, \& AudrainMcGovern, 2013; Patel \& Hu, 2008; Storfer-Isser, Patel, Babineau, \& Redline, 2012). Furthermore, it has been found that each additional hour of sleep is associated with decreases in BMI at different BMI precentiles among children (from 10th to 90th) (Mitchell et al., 2013).

In the adult literature, the association between sleep quality (good or poor sleep) and overweight or obesity is still unclear. Numerous studies have shown a strong association between short sleep duration and elevated BMI and increased risk for obesity among adults (Anic, TitusErnstoff, Newcomb, Trentham-Dietz, \& Egan, 2010; Bjorvatn et al., 2007; Cappuccio et al., 2008; Ford et al., 2014; Gangwisch, Malaspina, Boden-Albala, \& Heymsfield, 2005; Lauderdale et al., 2009; Marshall et al., 2008; Moraes et al., 2013; Patel \& Hu, 2008; Wheaton et al., 2011). However, Mezick, Wing, and McCaffery (2014) reported that shorter and less efficient sleep was associated with increased BMI among women only. On the contrary, Meyer, Wall, Larson, Laska, and Neumark-Sztainer (2012) found an existing association between sleep duration and BMI in men, but not women. Other cross-sectional studies either claim a relationship between long sleep duration and obesity, or no association between either short or long sleep duration and BMI (Magee \& Hale, 2012; Marshall et al., 2008; Nagai, Tomata, Watanabe, Kakizaki, \& Tsuji, 2013; Patel \& Hu, 2008; Xiang et al., 2009).

Among college students, although with obesity and lack of sleep highly prevalent in this group, conflicting results in the association between sleep behavior and BMI, overweight, and/or 
obesity also exist. Identical to studies with adults, the majority correlate short sleep duration with increased BMI, and increased risk for obesity. For example, Quick et al. (2013) examined the association between weight-related behaviors (i.e., eating, exercise), sleep duration (short: $<7$ hours, adequate: 7-8 hours, and long $>8$ hours), and BMI of college students ( $\mathrm{N}=1252 ; 18-24$ years) from nine U.S. Universities. The results showed that as sleep duration increased to adequate number of sleep, BMI declined and physical activity increased. This result supports the link between short sleep duration and increased BMI. Likewise, Chang and Chen (2014) in their examination of the association between poor sleep quality, BMI, and health-related physical fitness among college freshmen, found that poor sleep is associated with higher BMI and low performance of physical fitness.

In addition, among a sample of 207 college students enrolled at a large southeastern university, Todigala, Holcomb, and Kanneganti (2008), found that $42.8 \%$ of morbidly obese students and $50.1 \%$ of obese students reported usual sleeping hours of five or less. Similarly, Quick et al. (2013) found that poor sleep and sleep disturbances were significantly and highly prevalent among overweight and obese students. Moreover, Chen et al. (2013) reported in a cross-sectional study that staying up at night (few hours of sleep) increases the risk of being overweight. Likewise, in a prospective study of chronotype (morning [early bed- and wake time preferences], neutral, or evening types [late bed- and wake time preferences]) as predictors of increased weight gain and body mass index (BMI) among 137 college freshmen; Culnan, Kloss, and Grandner (2013) concluded that evening types had a significantly greater BMI gain $(\mathrm{p}<0.05)$ when compared with morning/neutral types. However, Vargas, Flores, and Robles (2014), included sleep disturbances in the examination of the association between sleep duration and BMI among a convenience sample of 515 college students. They concluded that only sleep 
disturbance, and not duration, was associated with overweight status (odds ratio 1.66, 95\% confidence interval $[1.08,2.57])$.

Similar to adults, other studies have found no association between sleep behaviors and BMI, overweight and or obesity among college students (Kamath et al., 2014; Melton, Langdon, \& McDaniel, 2013; Wuertz, Chia, \& Lee, 2012). Wuertz et al. (2012) investigated the relationship between BMI and sleep quality, and between physical activity and sleep quality among a convenience sample of 68 female students. Results indicated a significant association between poor sleep and low physical activity, but no association with BMI. In a cross-sectional analyses, no association was found between sleep duration and BMI (Kamath et al., 2014). In an investigation of students' sleep disturbances and key demographics (e.g., obesity classification, gender, race, year in college), sleep disturbance was not found to be a predictor of overweight or obesity (Melton et al., 2013).

Other studies have examined the relationship between sleep and eating behavior among college students. Quick et al. (2015) sugget that competent eaters (i.e., intra-individual approach to eating and food-related attitudes and behaviors) have better overall sleep quality and fewer sleep-related issues than less competent eaters. Accordingly, it has been concluded that poor sleep quality and low eating competence were associated with overweight/obesity. Among female college students, Makino, Hashizume, Yasushi, Tsuboi, and Dennerstein (2006) found that fewer sleeping hours is one of the most important factors associated with abnormal eating attitudes. In fact, poor sleepers suffer from high uncontrolled eating behaviors (Shoff et al., 2009). Uncontrolled eating behaviors can be explained by the fact that short sleep duration affects appetite regulatory hormones by decreasing leptin and elevating ghrelin, which lead to increased appetite (Taheri, Lin, Austin, Young, \& Mignot, 2004). In addition, Spaeth, Dinges, 
and Goel (2013) claimed that the weight gain due to sleep restriction (i.e., four hours in bed for a total of five consecutive nights) resulted from the consumption of extra calories particularly during late night hours. This is a metabolic explanation of the reported changes in BMI and risk for obesity due to short sleep duration. Essentially, sleep helps maintain a healthy balance of the hormones that induce hunger (ghrelin) or feelings of being full (leptin) (CDC, 2015).

\section{Significance and Purpose}

Among college students, the association between sleep quality (poor/good) and BMI, obesity or weight gain is still not sufficiently explored. The studies which have been conducted: 1) presented inconsistent findings, 2) have not controlled for important covariates, such exercise and diet restriction to lose weight, and 3) most studies have used convenience or selective samples. Therefore, additional investigation is warranted to examine the relationship between sleep behavior and BMI among college students with paying intention to important covariates. The main purpose of this study is to assess students' sleep quality in relation to BMI using a large national sample, with a long term objective of clearly supporting the literature that affirms the association between sleep and BMI, particularly among college students. Consequently, study results may inform strategies and /or intervention programs to enhance students' sleep habits and prevent weight gain and obesity among college students.

\section{Research Question, Aim, and Hypothesis \\ Research questions}

This aim seeks to answer the following questions:

\section{Primary Questions:}

1.1 Are there differences in sleep quality among the different BMI groups in a large sample of college students in the United States?

1.2 What is the association between sleep quality and BMI amongst a large 
sample of college students in the United States?

1.2.1 Does having more days of sleep quality impact BMI amongst a large sample of college students in the United States?

\section{Secondary Questions:}

2.1. What is the prevalence of poor sleep amongst a large sample of college students in the United States?

2.2. What is the prevalence of overweight and obesity amongst a large sample of college students in the United States?

\section{Aim(s) and hypotheses}

\section{Primary aims and hypotheses:}

1.1. To assess the differences in sleep quality amongst the different BMI categories amongst a large sample of college students in the United States.

1.2. To assess the association between sleep quality and BMI amongst a large sample of college students in the United States

1.2.2 To determine whether having more days of sleep quality increase the chance of decreasing or increasing BMI amongst a large sample of college students in the United States?

\section{Hypothesis:}

1.1 We expect an existence of differences in sleep quality among the different BMI categories, as poor sleep is expected to be prevalent in the higher levels of BMI classes (overweight and obesity) than underweight and desired weight groups. The expected differences may confirm the existence of association between sleep behavior 
particularly poor sleep and BMI, especially overweight and obesity classes amongst a large sample of college students in the United States.

1.2 We expect, based on the literature that increases in sleep quality may associated with decreases in BMI.

\section{Secondary aims and hypotheses:}

2.1. To assess the prevalence of poor sleep amongst a large sample of college students in the United States.

Hypothesis: poor sleep is expected to be prevalent amongst a large sample of college students in the United States.

2.2. To assess the prevalence of overweight and obesity amongst a large sample of college students in the United States.

Hypothesis: overweight and obesity is expected to be prevalent amongst a large sample of college students in the United States.

\section{Method}

\section{Study design, Data source and Population}

This study utilized the fall 2010-spring 2011 National American College Health Association-National College Health Assessment data (ACHA-NCHA). We were granted access to these data through a formal request to the American College Health Association. ACHANCHA provides the largest, most comprehensive data on the health of US college students. The participating institutions in this study include 42 and 129 postsecondary institutions who selfselected to participate in the fall 2010 and spring 2011 ACHA National College Health Assessment, respectively, totaling 30,263 (fall, 2010) and 116,254 (spring 2011) surveys completed by students. However, only schools that surveyed all students or used a random sampling technique were included in the analysis of ACHA-NCHA. Therefore, the final data set 
consisted of 30,093 (fall, 2010) and 105,781 (spring 2011) students for a total sample of 135,874 (ACHA, 2011). Nine health topics are covered in the survey including general health; diseases and injury prevention; academic impact; violence, abusive relationships and personal safety; alcohol, tobacco, and other drug use; sexual behavior; nutrition and exercise; mental health; and sleep (ACHA, 2011). Both surveys (Fall 2010 and Spring 2011) have 65 questions with sub questions that cover a variety of concepts related to the nine health topics. The overall response proportions were 30.9\%, and $28.5 \%$ for Fall 2010 and Spring 2011 respectively. Missing values (missing at random), n=2, 663 and 20, 419 individuals, were excluded from descriptive analysis and from inferential analysis, respectively.

\section{Instrumentation}

\section{Sleep Quality}

The study's independent variables measured aspects of sleep quality in the past seven days and in the past 12 months. Specifically, (in the past seven days), we assessed sleep quality as reflected by participants' experiences with: Enough Sleep, Early Awakening, Daytime Sleepiness (frequency), Difficulty Falling Asleep, and Problem with Daytime Sleepiness (magnitude). Two aspects of Daytime Sleepiness were assessed: the frequency of feeling sleepy during the day, and the magnitude of feeling sleepy (problem associated with sleepiness during the day). In the past 12 months, we assessed sleep quality as reflected by participants' experiences with Sleep Difficulties. The following questions were chosen to match the study aim: 1) Enough Sleep = "On how many of the past 7 days did you get enough sleep so that you felt rested when you woke up in the morning?"; 2) Early Awakening = "In the past 7 days, how often have you awaken too early in the morning and couldn't get back to sleep?"; 3) Daytime Sleepiness $=$ "In the past 7 days, how often have you felt tired, dragged out, or sleepy during the day?"; 4) Difficulty Falling Asleep = "In the past 7 days, how often have you had an extremely 
hard time falling asleep?"; 5) Problem with Daytime Sleepiness = "In the past 7 days, how much of a problem have you had with sleepiness during your daytime activities?"; and 6) Sleep Difficulties $=$ "Within the last 12 months, has any of the following been traumatic or very difficult for you to handle: Sleep Difficulties?" Response options for the first four questions ranged from zero to seven days; response options for question five was: $1=$ no problem at all, $2=$ a little problem, $3=$ more than a little problem, $4=\mathrm{a}$ big problem, and $5=$ a very big problem; and question six has yes or no response options.

For analysis purposes, particularly descriptive and Chi-square tests, we grouped the first four questions of sleep quality measure into three groups based on the literature (Becker, Adams, Orr, \& Quilter, 2008): Enough Sleep (6-7 days = optimal, 2-5 days = middle, and 0-1= poor). Early Awakening, Daytime Sleepiness, and Difficulty Falling Asleep were coded as: (6-7 days = poor, $2-5$ days $=$ middle, and 0-1= optimal). Problem with Daytime Sleepiness was coded as: (optimal $=$ having no problem with sleepiness, poor $=$ combining having a little, more than a little, a big, and a very big problem with sleepiness during the daytime activities).

\section{Body Mass Index (BMI) (dependent variable)}

BMI is the dependent variable that may be influenced by the independent sleep quality variables. BMI was calculated from the reported height and weight, and is a continuous variable. The calculation for computing BMI was weight $(\mathrm{kg}) /[\text { height }(\mathrm{m})]^{2}$. The continuous BMI variable was recoded into the following categories identified by the World Health Organization: Underweight (BMI <18.5), Healthy/Desired Weight (18.5-24.9), Overweight (25-29.9), Class I

Obesity (30-34.9), Class II Obesity (35-39.9), and Class III Obesity (BMI $\geq 40$ ). Specifically, two questions were designed to obtain height and weight: 1) "Height in feet and inches?", 
participants were asked to indicate their height in feet and inches; 2) "What is your weight in pounds?", participants were asked to indicate their weight in pounds.

\section{Covariates}

Study covariates (Tables 1b \& 1c; Appendix B) include gender, race, age, exercise, diet and pills to lose weight, Nonmedical use of prescription drugs [NMUPD] in the past 12 months (Antidepressants, painkillers, sedatives and stimulants), other substance use (i.e. cigarette use, alcohol use, and marijuana use in the past 30 days), diagnosed medical disorders (depression, insomnia, Attention deficit Hyperactivity Disorder (ADHD), sleep-related disorder), and overall perceived stress. These covariates were included because of known associations with sleep quality, and to control for any interrelated effects between the proposed covariates, poor sleep and BMI (Hershner \& Chervin, 2014; McCabe \& Teter, 2007; Tsai \& Li, 2004). The specific questions considered when adjusting for covariates with BMI follow:

Demographics: 1) "What is your gender?" (Male or Female); 2) "How do you usually describe yourself?", responses included: White-not Hispanic, Black-not Hispanic, Hispanic or Latino, Asian or Pacific Islander, American Indian or Alaskan, Biracial or Multiracial, and Others; and 3) "What is your age?".

Drugs/substance use: 1) "Within the last thirty days, on how many days did you use: Cigarettes?"; 2) "Within the last thirty days, on how many days did you use: Alcohol (beer, wine, liquor)?" 3) "Within the last thirty days, on how many days did you use: marijuana?". Responses were: Never used; have used, but not in last 30; 1 - 2 days; 3 - 5 days; 6 - 9 days; 10 - 19 days; 20 - 29 days; and/or used daily.

The NMUPD: 1) "Within the last 12 months, have you taken any of the following prescription drugs that were not prescribed to you: [Antidepressants (e.g., Celexa, Lexapro, 
Prozac, Wellbutrin, Zoloft)]; 2) "Within the last 12 months, have you taken any of the following prescription drugs that were not prescribed to you [Pain killers (e.g., OxyContin, Vicodin, Codeine)]; 3) "Within the last 12 months, have you taken any of the following prescription drugs that were not prescribed to you [Sedatives (e.g., Xanaz, Valium)]; 4) "Within the last 12 months, have you taken any of the following prescription drugs that were not prescribed to you [Stimulants (e.g., Ritalin, Adderall)]?" Response options to each question were no or yes. A separate set of questions asked about the medical use of those drugs of which data was not used in the present analyses.

Diagnosed medical disorders and the overall stress level: 1) "Have you ever been diagnosed with Depression?", responses for this question were no or yes; 2) "Within the last 12 months, have you been diagnosed or treated by a professional for any of the following: Insomnia?"; 3) "Within the last 12 months, have you been diagnosed or treated by a professional for any of the following: Attention Deficit and Hyperactivity Disorder (ADHD)?"; 4) "Within the last 12 months, have you been diagnosed or treated by a professional for any of the following: Other Sleep Disorder?", responses were: no; yes, diagnosed not treated; yes, treated with medication; yes, treated with psychotherapy; yes, treated with meds+ psychotherapy; and/or yes, other treatment. And for the overall perceived stress level the question was: "Within the last 12 months, how would you rate the overall level of stress you have experienced?" the responses were: no stress, less than average stress, average stress, more than average stress, and/or tremendous stress.

Exercise: 1) "On how many of the past 7 days did you: Do moderate intensity cardio or aerobic exercises for at least 30 minutes?; 2) "On how many of the past 7 days did you: Do vigorous intensity cardio or aerobic exercises for at least 20 minutes?" 3) "On how many of the 
past 7 days did you: Do 8-10 strength training exercises for 8-12 repetitions each?”; responses for those questions range from 0-7 days.

Exercise, diet and pills to lose weight: 1) "Within the last 30 days, did you do any of the following: Exercises to lose weight?"; 2) "Within the last 30 days, did you do any of the following: Diet to lose weight?"; 3) "Within the last 30 days, did you do any of the following: Vomit or take laxatives to lose weight?"; 4)_"Within the last 30 days, did you do any of the following: Take pills to lose weight?"; no or yes were the responses options.

\section{Data Analysis}

Descriptive statistics were followed by Chi-square, ordinal regression models, and linear regression models, to examine the associations between sleep quality (in the past seven days, and past 12 months (Enough Sleep, Early Awakening, Daytime Sleepiness, Difficulty Falling Asleep, Problem with Daytime Sleepiness, and Sleep Difficulty) as ordinal and binary variables and BMI (as categorical and continuous variables). Chi-square was used to compare BMI categories in term of sleep quality particularly poor sleep, followed by multivariate ordinal regression to test the association between sleep quality and the categorical BMI variables, and multivariate linear regression to test the association between sleep quality and the continuous BMI variable while controlling for the study covariates using SAS 9.4. We excluded gender from the study covariates when modelling by gender. The effect size was measured by $\mathrm{R}$ value, which is the square root of $\mathrm{R}^{2}$; correlation was judged as $(\mathrm{R}=.1$ : small effect, $\mathrm{R}=.3$ : moderate effect, and $\mathrm{R}=.5$ : large effect). 
Results

\section{Sample characteristics}

Table 1 and Figures $1 \& 2$ (Appendix B) present sample characteristics. Participants included 86,593 females (65.3\%) and 46,062 males (34.7\%) students (Mean age=22.0, SD=6.0). Students were primarily White (68.1\%). However, the lowest presentation of ethnicity has been recorded for American Indian, Alaskan Native, or Native Hawaiian (1.6\%) (Table 1a). The majority of students $(65.6 \%)$ reported using alcohol in the past 30 days; whereas $15 \%$ and $15.4 \%$, respectively, reported cigarette smoking and marijuana use in the past 30 days (Table 1b).

Table 1b, also illustrates the frequencies and percentages of NMUPD [four classes: Antidepressants, Painkillers, Stimulants, and Sedatives (in the past 12 months)], the diagnosed medical disorders (in the past 12 months), and the overall perceived stress level. Approximately $14 \%$ of students reported the nonmedical use of at least one class of prescription drugs. The highest usage rates have been reported for painkillers $(7.5 \% ; n=9,826)$, followed by $7.4 \%$ $(n=9,661)$ for stimulants. However, only $4 \%(n=5,338)$ reported sedatives use, and 3\% $(n=4,006)$

reported antidepressants use. Gender differences reveal that females have higher usage rates than males for all the classes; almost as twice as males. For example, $68.5 \%$ of antidepressants users were females, compared to $31.5 \%$ males (Figure $2 b$ ).

Most students (98.3\%) reported stress level of average and above; and approximately $18 \%$ reported being diagnosed with depression (Table $1 \mathrm{~b}$ ). In addition, in the past 12 months, $4.4 \%$ of students reported being diagnosed and/or treated for ADHD, 3.7\% for insomnia, and only $2 \%$ of students have been diagnosed and/or treated for sleep-related disorders (Table 1b). A 
Females who have reported stress, depression and have been diagnosed and/or treated for ADHD, insomnia and sleep-related disorders were higher than males, almost double (Figure 2c).

Table 1c, shows three different levels of exercise behaviors (moderate intensity, vigorous intensity, and strength training exercises) practiced by students in the past 7 days; and four different ways used by students to lose weight in the past 30 days (exercises, diet, vomit or taking laxatives, and taking pills). Exercising for 2-5 days/week was reported by most students for the three different levels of exercises. Among students who did moderate intensity cardio or aerobic exercises for at least 30 minutes in the past 7 days, $52.8 \%$ exercised for 2-5 days, while only 10\% did for 6-7 days. Vigorous intensity cardio or aerobic exercises for at least 20 minutes in the past 7 days, have been reported by $39.6 \%$ students for $2-5$ days, and only $5.4 \%$ of students who exercised for 6-7 days. In addition, $32 \%$ of students practiced 8-10 strength training exercises for 8-12 repetitions each for 2-5 days/week, and just 3.3\% who did for 6-7 days/week. Females in general had more days of exercises of at various intensity than males, except of doing 8-10 strength training exercises for 8-12 repetitions each for 6-7 days, $54.7 \%$ were males compared to $45.3 \%$ females (Figure 2d). The most common reported ways of losing weight were exercise and diet (54\% and 38\% respectively) (Table 1c). However, using pills, vomiting or taking laxatives to lose weight were also reported by $3.4 \%$ and $2 \%$ (respectively) of students. Females were more likely than males in using these weight lose strategies (Figure 2e).

\section{Sleep Quality and the Body Mass Index (BMI) description}

Table 2 and Figure 3 present participants' experiences of six different aspects of sleep quality (in the past 7 days and past 12 months), and the overall BMI and BMI classifications distribution among students. In the past 7 days, about $22 \%$ of students reported not getting Enough Sleep (0-1 day/week); 24.3\% reported having 2-5 days/week of Early Awaken; 62.3\% 
Daytime Sleepiness for 2-5 days/week; and about 28.5\% of students had Difficulty Falling Asleep for 2-5 days/week. However, majority of students (90\%) reported having Problems with Daytime Sleepiness in the past 7 days.

Among all the six aspects of sleep quality, the proportions of females are higher than males (Figure 3a). For example, females reported less days of having Enough Sleep and more days of Daytime Sleepiness (72.7\%) than males (30.4\% and $27.3 \%$, respectively). In addition, having 6-7 days of Early Awakening and 6-7 days of Difficulty Falling Asleep were higher among females (69.4\% and 69\%, respectively) than males (30.6\% and 31\%, respectively). Problem with Daytime Sleepiness was also higher among females (66.6\%) than males (33.4\%).

The overall BMI was 24.2 ( $\mathrm{SD}=5.1)$; Table 2 and Figure 3e illustrate the distribution of six BMI classifications (underweight, desired weight, overweight, and three classes of obesity). Almost a third of students classified as overweight and obese (Figure 3e) distributed as $21.3 \%$ overweight and $11.1 \%$ obese students (collectively of the three obesity classes). Distribution of the BMI classes by gender shows that females have higher percentages than males in all classes (Table 3d). Among overweight class there were $55.4 \%$ females compared to $44.6 \%$ males. In addition, there were more obese females particularly in obesity class $3(72.2 \%)$ than males $(27.8$ $\%)$.

\section{The association between sleep and BMI}

\section{Differences in sleep quality among the different BMI classes}

The Chi-square test of association (Table 3 a \& b Appendix B) reveals significant bivariate relationships between the different sleep quality aspects and BMI: [Enough Sleep $\left(\chi^{2}\right.$ $(10)=308.3, \mathrm{p}<.0001)$, Early Awakening $\left(\chi^{2}(10)=546.0, \mathrm{p}<.0001\right)$, Daytime Sleepiness $\left(\chi^{2}\right.$ $(10)=425.7, \mathrm{p}<.0001)$, Difficulty Falling Asleep $\left(\chi^{2}(10)=678.0, \mathrm{p}<.0001\right)$, Problem with 
Daytime Sleepiness $\left(\chi^{2}(10)=11.9, \mathrm{p}<.05\right)$, and Sleep Difficulty $\left(\chi^{2}(5)=403.9, \mathrm{p}<.0001\right)$. Students who reported less days of Enough Sleep (0-1 day/week) were more often in the obesity classes and overweight categories than desired weight. Around 30\% of class 3 obesity, $27.6 \%$ of class 2 obesity, $25.5 \%$ of class 1 obesity, and $22.6 \%$ of overweight were more likely to be poor sleepers (0-1 day/week of Enough Sleep) compared to $20.1 \%$ of desired weight group. Having more days (2-5 days/week) of Early Awakening were reported by $30 \%$ of obesity class 3 students, followed by obesity class 2 (29.7\%), obesity class 1 (28.9\%), and overweight (25.3\%) students, compared to the desired weight group (23\%).

Daytime Sleepiness for 6-7 days/week, were reported by $25.7 \%$ of class 3 obesity, $22.4 \%$ of class 2 obesity, and $20 \%$ of class 1 obesity students, compared to only $15 \%$ of students in the desired weight group. However, there was no significant differences among the BMI groups in relation to having a Problem with Daytime Sleepiness. Obesity classes also have higher proportions of students reported having 2-5 days/week of Difficulty Falling Asleep (32.4\%, $31.4 \%$, and $31.8 \%$, class 3,2 and 1 respectively) than other groups particularly the desired weight group (27.8\%). Finally, around $26.0 \%, 30.0,33.0 \%$, and $38.0 \%$ of overweight, obesity (class1, 2 and 3) respectively reported having Sleep Difficulties in the past 12 months.

Analysis by gender indicated that, in general, there were more females in each BMI group who reported poor sleep quality than males; except among class obesity 3 , there were more male (33.1\%) who reported 2-5 days/week of having Difficulty Falling Asleep than females $(31.9 \%)$. 
The association between the different sleep quality aspects and BMI measures (multivariate ordinal regression and Linear Regression analyses)

Results of the ordinal regression analysis (Odds Ratio, [95\% Confident Interval]) are presented in Table 4a (Appendix B). Overall significant associations were found between sleep quality and BMI classes, even after adjusting for covariates. Students who reported having Problem with Daytime Sleepiness (1.05 [1.00-1.10]), and Sleep Difficulties (1.05 [1.01-1.08]) were significantly more likely to be in the higher BMI classes (overweight and obesity) than those who did not. In addition, students who reported having more days of Enough Sleep (0.98 [0.98-0.99]) were less likely to be overweight and/or obese. However, students who reported more days of Early Awakening, Daytime Sleepiness, and Difficulty Falling Asleep also were significantly more likely to be overweight and obese 1.01(1.00-1.02), 1.03 (1.02-1.04), and 1.04 (1.03-1.05) (respectively), compared to those who reported less days.

Gender differences were found in relation to four aspects of sleep quality. Problem with Daytime Sleepiness and Sleep Difficulties were significantly associated with the likelihood of being in the higher levels of BMI categories ( $\mathrm{OR}=1.08$, and 1.05; respectively) among females only. Among males, having more days of Enough Sleep was significantly associated with less chances of being in the higher levels of BMI categories (OR=0.98). Also, among males only, students who reported having more days of Early Awakening were significantly more likely to be in the higher levels of BMI categories $(\mathrm{OR}=1.02)$ than those who reported less days.

Results of the multivariate linear regression analysis (Table 4b: Appendix B) reveal a significant overall relationship between sleep quality and BMI $(\mathrm{F}(29,115425)=372.5, \mathrm{p}<0001)$ with an $\mathrm{R}^{2}$ of 0.086 ; even after controlling for covariates. The model explained $8.5 \%$ of the variance in BMI (effect size is $\mathrm{R}=0.3$, which is moderate). Students' BMI decreased by 0.02 units for each day increased in getting Enough Sleep. BMI would increase by 0.03 units, for each 
day increases in getting Early Awakening. When the number of days of Daytime Sleepiness increases by one day, the average BMI is predicted to increase by 0.10 units. And for each day increases of having Difficulty Falling Asleep, BMI increases by 0.11 units. The predicted BMI would be 0.11 units higher for students who had Problem with Daytime Sleepiness than those who do not. Finally, the BMI is predicted to be 0.13 units higher for students who reported having Sleep Difficulty in the past 12 months than those who do not have.

When analyzing by gender we found variations with the effects of the following sleep quality aspects. The effects of having Problem with Daytime Sleepiness, and Sleep Difficulty on BMI were significant among females only. The BMI is predicted to be 0.22 and 0.16 higher for female students who had Problem with Daytime Sleepiness and reported Sleep Difficulty (respectively) than those who do not. However, having more days of Early Awakening is significant only among males; the BMI would increase by 0.04 units for each day increases in Early Awakening.

\section{Discussion}

This is the first known study to examine the association between six aspects of sleep quality [Enough Sleep, Early Awakening, Daytime Sleepiness, Difficulty Falling Asleep, and Problem with Daytime Sleepiness (in the past seven days), and Sleep Difficulties (in the past 12 months)], and BMI using a large national sample of college students. The current known studies focus on sleep duration (number of hours/days of getting enough sleep). Results revealed that poor sleep is prevalent among college students in our sample as reflected by six aspects of sleep quality. The majority of students (90\%) reported Problems with Daytime Sleepiness, 69.4\% reported Early Awakening, $28.5 \%$ reported Difficulty Falling Asleep, $24.8 \%$ reported having Sleep Difficulty, and 22\% of students had few days of Enough Sleep/week. Correspondingly, the 
literature affirmed the prevalence of poor sleep among college students, with more than $60 \%$ classified as poor quality sleepers (Tsai \& Li, 2005; Lund et al., 2010). Female students in our sample reported poorer sleep in each aspect of sleep quality, supporting previous research suggesting females report poorer sleep quality than males (Tsai \& Li, 2005).

Overweight and obesity were also prevalent among students in our sample, almost one third of students were classified as overweight and obese. Previous studies reported that more than two-thirds of U.S. adults are considered to be overweight or obese (CDC, 2015a; NIDDK, 2012; NIH, 2012b).

Poor sleep has been consistently connected with higher BMI (overewight and obesity), in children and adolescents (Magee \& Hale, 2012; Marshall, Glozier, \& Grunstein, 2008; Mitchell, Rodriguez, Schmitz, \& Audrain-McGovern, 2013; Patel \& Hu, 2008; Storfer-Isser, Patel, Babineau, \& Redline, 2012). In adult literature, although the association is still unclear and inconsistent, several studies have shown a strong association between poor sleep quality (particularly shorter sleep duration) and elevated BMI and increased risk for obesity (Anic, TitusErnstoff, Newcomb, Trentham-Dietz, \& Egan, 2010; Bjorvatn et al., 2007; Cappuccio et al., 2008; Ford et al., 2014; Gangwisch, Malaspina, Boden-Albala, \& Heymsfield, 2005; Lauderdale et al., 2009; Marshall et al., 2008; Moraes et al., 2013; Patel \& Hu, 2008; Wheaton et al., 2011). Consistent with study expectations and current literature, our findings also confirm the existence of significant association between poor sleep quality (short and long term) and BMI, even after controlling for key covariates.

Chi-square results show differences among the different BMI categories in terms of poor sleep quality of six sleep aspects. At least a quarter of obesity class 1, class 2, class 3, and overweight students reported having Sleep Difficulties in the past 12 months, less days of 
Enough Sleep (0-1 day/week), more days (2-5 days/week) of Early Awaken, more days of Daytime Sleepiness for 6-7 days/week, and more days (2-5 days/week) of having Difficulty Falling Asleep. In line with the literature, poor sleep quality was prevalent among overweight and obese students. Todigala, et al (2008) found that students who reported usual sleeping hours of five or less per night were high among overweight and obese classes. In addition, Quick et al. (2013) found that poor sleep and sleep disturbances (i.e Early Awakening, Difficulty Falling Aleep, Daytime Sleepiness, and Sleep Difficulties) were significantly and highly prevalent among overweight and obese students.

The multivariate regression models (ordinal logesitic and linear) also confirmed the association between the six aspects of sleep quality and BMI, even after controlling for key covariates. However, the overall effect is significantly weaker once the covariates are included in the model. Overall, poor sleep qulity was significantly associated with overweight and obesity in the logistic regression analysis. Most significantly (with the highest odds), the likelihood of being overweight or obese is higher for studnets who had Problem with Daytime Sleepiness (in the past seven days) and had Sleep Difficulties (in the past 12 months) than those who did not have. In addition, students who had more days of Enough Sleep were less likely to be overweight and/or obese. Students who reported more days of Early Awakening, Daytime Sleepiness, and Difficulty Falling Asleep, although the odds were small, they still significantly have higher chances of being overweight and /or obese compared to those who reported less days. In the linear regression analysis poor sleep quality was conncetd to increases in the BMI measures. While having more days of enough sleep (good sleep quality) was associated with decreases in BMI. Increasing in the number of days of Daytime Sleepiness, Difficulty Falling Asleep, Early 
Awakening were connected with increasing in BMI. In addition, increase in BMI among students was also related to having Problem with Daytime Sleepiness and Sleep Difficulty.

Although, the current literature among college students have conflicting results in the association between sleep quality and BMI, our findings support the literature that correlates short sleep duration with increased BMI, and increases risk for overweight and obesity (Anic, et al. 2010; Bjnorvatn, et al. , 2007; Chang and Chen, 2014; Culnan, et al., 2013; Gangwisch et al, 2005; Ford, et al, 2014; Mitchell et al., 2013; Mezick, et al., 2014; Quick et al., 2013). For example, Ford, et al (2014) reported that short sleep duration was associated with increased BMI, and short sleepers were more likely to be obese. Similarly, Chen et al. (2013) reported that having few hours of sleep increases the risk of being overweight. Mitchell et al. (2013) and Quick et al. (2013) concluded that as sleep duration increased, BMI declined. In addition, the association between sleep disturbances (including Early Awakening, Daytime Sleepiness, Difficult Falling Asleep, Problem with Daytime Sleepiness, and Sleep Difficulty) and the increased BMI in our results, is in line with, Chang and Chen (2014), Mezick, et al., (2014), and Quick et al. (2013) Vargas, Flores, and Robles (2014). For example, Quick et al. (2013) reported that poor sleep quality and sleep disturbances were significantly and highly prevalent among overweight and obese students. Chen et al. (2013) concluded that few hours of sleep per night increases the risk of being overweight. And finally, Vargas, et al. (2014), pronounced that only sleep disturbances, and not duration, were associated with overweight. 
Interestingly, we have found variation in the association between sleep quality and BMI when analysing by gender consistently in both regression models. Problem with Daytime Sleepiness and Sleep Difficulties were significantly associated with the risk of being overweight and obese among females only. A possible explanation for this variation may be related to the fact that in our results, there were more females than males who reported having Problem with Daytime Sleepiness and Sleep Difficulty. In addition, more females were in the overweight and obesity classes than male. However, having more days of Enough Sleep was significantly associated with less chances of being overweight and obese for males but not females. Also, among males only, students who reported having more days of Early Awakening were significantly more likely to be overweight and obese than those who did not. Gender variation in our results concurs with previous reports. Conflicting results have been noted in the literature regarding gender differences. Mezick, et al., (2014), Anic, Titus-Ernstoff, Newcomb, TrenthamDietz, \& Egan, (2010), claimed the association between poor sleep quality and BMI, overweight and/or obesity to be among female only as opposed to Meyer, et al., (2012) \& Wuertz et al. (2012).

In fact, there is a metabolic explanation of BMI changes and risk for obesity due to short sleep duration. Essentially, good sleep quality helps maintain a healthy balance of the hormones that induce hunger (ghrelin) or feelings of being full (leptin) (CDC, 2015). Poor sleep (particularly short sleep duration) affects appetite regulatory hormones by decreasing leptin and elevating Ghrelin, which leads to increased appetite (Taheri, Lin, Austin, Young, \& Mignot, 2004). In addition, Spaeth, et al. (2013) claimed that the weight gain due to poor sleep duration is related to the consumption of extra calories, particularly during late night hours. In general, it has been asserted that poor sleepers suffer from high uncontrolled eating behaviors (Shoff et al., 
2009). This fact introduce eating behavior as an important factor that may play a mediator role in the association between sleep quality and BMI. However, data regarding eating behavior was not availabel in the secondary data we analysed, we recommended to be considered in future studies.

Finally, we were able to include weight loss behaviors (exercises, diet, vomit or take laxatives, and take pills) among students as covariates in the regression model. As a result, we found with no significant association between sleep quality and BMI except for Daytime sleepiness and Difficulty Falling Asleep. The BMI would be increased by 0.08 and 0.09 units for each day increases of having Daytime Sleepiness and Difficulty Falling Asleep (respectively). The effects of the other sleep quality aspects on BMI may be hindered by including weight loss behaviors which may explain the mederator effects of losing weight. These may have effects on sleep quality in addition to the direct relationship with BMI.

\section{Limitation}

Study limitations are noted here. First, we utilized a cross-sectional research design, thus, causal relationships between study variables cannot be determined. Second, because results are based on self-reported data, potential recall bias including participants' desire to report socially desired behaviors and reluctance to report undesirable behavior may occur. Third, this study relies upon the subjective measure of sleep behavior and not on objective measures such as polysomnography which provides a more accurate and holistic picture of sleep. Finally, since colleges self-select to participate in the ACHA survey, results cannot necessarily be considered generalizable to the US college population. Therefore, further investigation of the association between sleep quality and BMI among college students that take into consideration the identified limitation is recommended. 


\section{Conclusion}

In conclusion, poor sleep was common in this sample of college students and study results suggest that poor sleep (short and long term) quality significantly increases the predicted values of BMI and increases the risk of being overweight and obese. Our study extends the current literature that support the association between poor sleep quality (including short sleep duration) and elevated BMI. Therefore, investigations or interventions concerning college student overweight/obesity should include screening for sleep quality to promote healthy weight. Study results also highlight gender differences that should also be incorporated into efforts to better understand and to improve college students' health, sleep health, productivity, wellness, quality of life, and academic achievement. 


\section{A Theory-based Description of Sleep Quality and Its Impact on Health-Related Quality Of Life Using Health Belief Model and Behavioral Intention among a Sample of WVU Undergraduates Students}

Introduction

\section{Health Belief Model and Theory of Planned Behavior}

The Health Belief Model (HBM) and the Theory of Planned Behavior (TPB) are two of the most common social and behavioral theories used to explain and/or predict health-related behavioral determinants, and guide the development and implementation of health promotional and educational interventions (Painter, Borba, Hynes, Mays, \& Glanz, 2008). Social and behavioral theories can play a significant role in changing behaviors, and it has been demonstrated that public health interventions based on theories are more effective and powerful than those without (Glanz \& Bishop, 2010; Glanz, Rimer, \& Viswanath, 2008). Both the HBM and TPB have been used widely to understand behaviors and to inform interventions; both theories have been supported for their ability to successfully predict behaviors in a wide range of health behaviors including, for example, HIV prevention behaviors, mammography, immunization, screening, dieting, smoking, and substance use (Abood, Black, \& Feral, 2003; Bashirian, Hidarnia, Allahverdipour, \& Hajizadeh, 2012; Glanz \& Bishop, 2010; Glanz et al., 2008; Painter et al., 2008; Taylor et al., 2006).

\section{Theory based description/prediction of sleep quality}

Sleep quality (good/healthy or poor sleep) are outcomes of sleep practice (sleep

behavior). Sleep behavior (also called sleep hygiene) refers to practicing behaviors that facilitate sleep, in order to have normal, quality nighttime sleep and full daytime alertness (NSF, 2015; Riedel, 2000). Therefore, promoting good sleep quality should be based on strategies directed at 
sleep behaviors including applying social and behavioral theories to describe, predict, and or promote healthy sleep practice. However, to the best of the authors' knowledge, sleep research lacks the explanation and prediction of sleep quality, and thus potential interventions based on contemporary science. Extant studies using both theories primarily focus on sleep disorders as opposed to sleep hygiene and behaviors. A review of those studies follows in the literature section of this paper.

\section{Significance and Purpose}

The sleep literature is still indigent in terms of the application of both TPB and HBM and few studies have theoretically described sleep quality among college students using both theories (e.g., Knowlden \& Sharma, 2014). In addition, there is also no evidence of an integrated behavioral model using constructs from both HBM and TPB to describe and promote sleep quality among adults or college students. Thus, there is a dearth of health behavior theoretical research exploring the behavioral determinants of sleep among college students. It is crucial to theoretically examine and describe sleep in order to better understand sleep quality, their behavioral determinants, and predict sleep related problems among college students. Such research may lead to improved understanding and establish appropriate strategies and/or interventions to enhance students' sleep quality.

This is the first known study that will use an integrated theoretical framework to assess sleep quality among college students. The main purpose of this study is to describe college students' sleep and their behavioral determinants using behavioral constructs from both the TPB and HBM as an integrated theoretical framework. Additionally, this study will assess the association between sleep quality, particularly poor sleep and health related quality of life [HRQOL] (as physical and mental health perceptions and their correlates) among a sample of 
college students from West Virginia University (WVU).

\section{Research questions, Aims, and Hypotheses \\ Research questions,}

This research is specifically guided by the following questions:

\section{Primary Question:}

1.1. Do HBM including perception constructs, and the the behavioral intention of TPB describe and/or predict students' sleep quality, amongst a sample of WVU college students?

\section{Secondary Questions:}

2.1. What is the association between sleep quality and HRQOL amongst a sample of WVU college students?

2.2. What is the prevalence of poor sleep quality amongst a sample of WVU college students?

\section{$\operatorname{Aim}(s)$ and hypotheses:}

\section{Primary aim and hypothesis:}

1.1. To explain/predict WVU students' sleep habits using the HBM perception constructs: (perceived susceptibility, perceived severity, perceived barriers, and perceived benefits), and the behavioral intention from the TPB, as an integrated framework.

Hypothesis: Students' sleep behavior can be described and or predicted by HBM and behavioral intention constructs.

\section{Secondary aims and hypotheses:}

2.1. To examine the association between poor sleep quality and HRQOL as reflected by measuring unhealthy days amongst a sample of WVU college students.

Hypothesis: poor sleep quality is associated with having more days of unhealthy days which 
reflect poor HRQOL.

2.2. To assess the prevalence of poor sleep amongst a sample of WVU students.

Hypothesis: Poor sleep is prevalent among WVU students.

\section{Description of the proposed theoretical framework}

Elements of the framework

The framework/model consists of the folowing:

- External factors (Gender, Race, and Year of enrollment).

- HBM constructs (perceived threat, perceived benefits, and perceived barriers).

- TPB construct (behavioral intention).

- Sleep Quality (good or poor sleep).

- Unhealthy Days (HRQOL).

Sleep behavior among college students can be determined by:

- Perception, including perceived susceptibility of being poor sleepers, perceived severity of poor sleep and its associated health outcomes, perceived benefits of having a good night sleep, the barriers of getting good sleep (HBM)

- The perceived likelihood of performing a healthy sleep behavior (behavioral intention) (the TPB construct).

- In addition, the proposed framework comprises some of the important factors considered as external variables to be controlled; gender, race, and year of enrollment. These constructs have been integrated in the theoretical framework to assess their contribution to sleep quality and HRQOL among college students; in order to, explained and predicted students' sleep. The model is hypothesized to describe the behavioral determinants of

sleep quality; therefore, two theories have been integrated in the model. Figure 1 illustrates how sleep behavior can be influenced by those behavioral constructs in addition to some important 
external factors, leading to either good or poor sleep quality, and their impacts on HRQOLUnhealthy days.

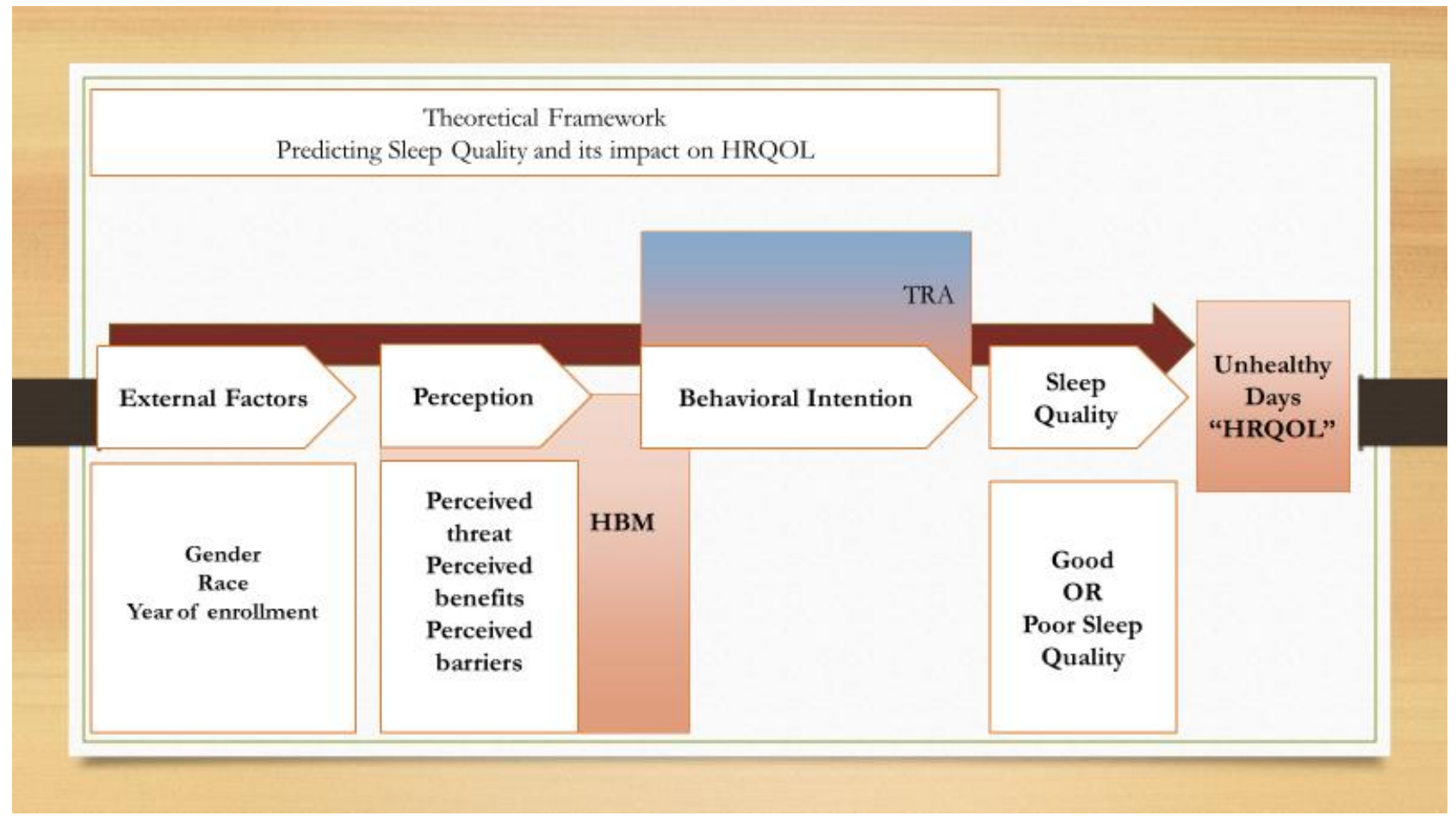

Conceptual and operational definitions of the Model's elements:

\section{External factors}

Demographic variables are considered as modifying factors that may indirectly affect the desired behavior such as sleep by influencing the constructs of perception (threat, benefits, and barrier) and behavioral intention, and then indirectly affect others in the model.

\section{HBM Constructs}

Perception includes perceived threat (susceptibility \& severity), perceived benefits, and perceived barriers. Perceived threat is a combination of both perceived susceptibility and perceived severity. Perceived susceptibility or perceived vulnerability refers to ones' own belief and opinion about the possibility of getting an illness or condition (e.g. poor sleep quality) (Glanz et al., 2008). Perceived severity is the perception of the significance, seriousness and consequences of getting an illness, or leaving it untreated (e.g. poor sleep quality), including 
evaluation of medical cost (pain, disability, or death), and social costs (effects on work, school, family and social relations) (Glanz et al., 2008). Perceived benefits refers to the belief of the potential benefits of the recommended action (e.g. healthy/good sleep) in reducing the risk or severity of potential disease (reducing threat). Benefits may involve medical, financial, and social and family lives (Glanz et al., 2008). Finally, perceived barriers refer to the perception of the potential negative aspects of a particular health behavior/action including financial and psychosocial costs (Glanz et al., 2008).

Operationally, these constructs are defined according to the definitions developed by Knowlden and Sharma (2014), with some modifications based on the purpose of this study. Originally, the HBM constructs were operationalized in relation to adequate sleep behavior. However, for this study they will be operationalized in relation to sleep quality, which refers to both adequate sleep (getting the recommended sleeping hours), and aspects of sleep quality as reflected by the Pittsburgh Sleep Quality Index [PSQI] (Buysse et al.,1989). The PSQI is considered as the "gold standard".

Perceived susceptibility is operationalized in terms of both the short- and long-term outcomes of poor sleep such as sleep disorders (e.g. sleep apnea, insomnia, narcolepsy, and restless legs syndrome); several negative behavioral and health outcomes (including substance use, physical inactivity, violence, mental disturbances, suicide attempts, overweight or obese status, chronic diseases including heart disease, drowsy driving, and risk for accidents and injuries); and poor school performance and lower average school grades. Accordingly, it can be defined as a student's own belief and opinion about the possibility of getting one or more of the associated negatives outcomes. Perceived severity is operationalized within the context of the personal vulnerability to the short- and long-term outcomes of poor sleep. Hence, it can be 
defined as student's perception of the significance, seriousness and consequences of getting one or more of the associated negative outcomes.

The construct of perceived benefits is operationalized in terms of student's viewpoints of the personal short and long-term rewards in relation to health, energy levels, and school productivity acquired from achieveing good sleep quality. It can be defined as student's belief of the potential rewards of practicing healthy sleep in avoiding or reducing the risk or severity of the potential negative outcomes. Finally, the construct of perceived barriers operationally refers to the perspective pressures that are considered as obstacles for achieving good sleep quality, such as academic and employment responsibilities.

\section{Behavioral Intention (TPB Construct)}

Behavioral intention is the perceived likelihood of carrying out the behavior (Glanz et al., 2008). Behavioral intention is operationalized according to Knowlden (2011), and willo be measured as the intention to perform healthy sleep behavior to obtain good sleep quality.

\section{Sleep Quality}

Sleep quality is an overall outcome of sleep habits/behaviors as either good or poor sleep quality. Sleep quality among adults can be defined as getting the recommended 7-8 hours of sleep (sleep duration) with good indices of sleep quality per night (CDC, 2013; NIH, 2012a). However, poor sleep quality can be explained as getting less or more than the recommended range of sleeping hours between 7 and 8, with poor indices of sleep quality per night (CDC, 2013; NIH, 2012a). Sleep quality indices, as an important criterion of the overall sleep quality, can be defined as subjective indices of sleep, and largely include the following concepts or aspects: falling asleep and sleep latency, depth of sleep, how well rested one feels upon awakening, problem with sleepiness during daytime activities (such as feeling tired), daytime 
sleepiness, awakening time (number of arousal), and the general satisfaction with sleep (Buysse et al., 1989).

\section{Health-related quality of life}

In general, quality of life is a broad concept that incorporates all aspects of life including an overall sense of well-being, aspects of happiness, and satisfaction with life as a whole (CDC, 2000, 2011a). There are several key aspects that constitute the overall quality of life, including

for instance health, jobs, housing, schools, the neighborhood, and aspects of culture, values, and spirituality (CDC, 2000). In this study, we assessed HRQOL (physical and mental health perceptions and their correlates). HRQOL was defined as an individual's or group's perceived physical and mental health over time (CDC, 2011a).

\section{Model assumptions}

The model assumes a relationship between the external factors, the constructs, sleep quality, and HRQOL, as shown in Figure 1, (p 56). The effect on the desired behavior (sleep quality) that needs to be developed, modified or changed starts with the external factors. External factors such as demographics may directly influence perceptions (threat, benefits, and barrier). In turn, perceptions may directly or indirectly (behavioral intention) impact sleep quality. Directly, student's perception of being at risk of getting sleep related disorder and the associated complications (perceive threat), can lead one to consider the benefits of taking actions including avoiding the threat (e.g., avoiding sleep disorders when developing healthy sleep behaviors), and getting other positive outcomes such as having a healthy and active lifestyle. Perceived threats and perceived benefits of taking a particular sleep behavior, may lead to outweighing the potential benefits associated with good sleep quality, with the factors that prevent from getting good sleep quality (such as sleep related factors, work/study load, time, and/or being unpleasant to go early to bed). Indirectly, the influence of perception may be mediated through behavioral 
intention. Students' intentions to perform healthy sleep behavior or not will be affected by their perceptions. Finally, good and poor sleep may impact students' HRQOL.

\section{Literature Review}

\section{Model's external factors and sleep (first research question)}

\section{Race/Ethnicity}

Different ethnic groups exhibit different sleep behaviors. For example, Ruiter, Decoster, Jacobs, and Lichstein (2011) found significant ethnic differences in relation to good sleep quality in a meta-analysis of an adult population. African-Americans have poorer sleep continuity and duration, less stage 3 sleep (NREM 3: slow wave sleep or deep sleep), and a greater proportion of stage 2 sleep (NREM 2: light sleep) than Non-Hispanic Whites (NHW). Moreover, Mezick et al. (2008) examined the influence of race and socioeconomic status on sleep among adults, and found that African-Americans had shorter sleep duration and efficiency, and may be at risk for sleep disturbances and associated health consequences. In addition, Grandner et al. (2010) reported in their assessment of the general sleep complaints (reporting trouble falling asleep, staying asleep or sleeping too much), that Asians had the least rate of complaints, while NHWs have more sleep complaints. The rate of sleep complaints of Hispanic/Latino and AfricanAmericans were more than Asians and less than NHWs. Conversly, it has been stated that African American and Asian students sleep better than White and Latino students (Gaultney, 2010). Phillips and Mannino (2005) asserted that African-Americans have reduced risk of sleep complaints. Moreover, according to the CDC (2015), short sleep duration was found to be more common among non-Hispanic Blacks compared to non-Hispanic Whites.

\section{Gender}

Studies that examine gender differences in relation to sleep report contradictory findings. The majority of literatures state that women sleep longer than men, but with lower sleep quality 
(Burgard, Ailshire, \& Hughe, 2010; Burgard \& Ailshire, 2012; Natal et al., 2009; Tsai \& Li, 2004). Among college students, female students report earlier bed time and getting up, longer sleep latency and sleep duration, more awakenings, and poorer sleep quality than males (Sweileh et al., 2012; Tsai \& Li, 2004). Furthermore, Wuertz et al. (2012) concluded that sleep problems are highly prevalent among college women. However, Ohayon (2004) found that among the elderly (between 55 and 101years old) sleep duration was longer in men than in women. In addition, Oginska and Pokorski (2006) found that females reported a greater need of sleep than males, and suffer from a higher level of daytime sleepiness; consequently, they may be more severely affected by chronic sleep loss than males.

\section{SES (for both research questions)}

Socioeconomic status has been found to be strongly associated with both subjective and objective sleep quality (Friedman et al., 2007). Higher household income and more years of education have been found to be significantly associated with reduced sleep latency (time taking to fall asleep) and increased sleep efficiency (refers to the quality of sleep) (Friedman et al., 2007). However, lower socioeconomic status has been connected to sleep complaints (as defined as trouble falling asleep and longer sleep latency, wake up repeatedly during the night, and wake up with a feeling of exhaustion and fatigue), and poorer sleep quality (Mezick et al., 2008; Phillips \& Mannino, 2005). In an examination of the general sleep complaints (reporting trouble falling asleep, staying asleep or sleeping too much), Grandner et al. (2010) found that lower income is related to more reported sleep complain. In addition, children from low-SES families have been found to have poor sleep quality due to parental depression and family conflicts (Buckhalt \& El-Sheikh, 2013). Likewise, Mezick et al. (2008) affirm that individuals in lower SES groups may be at risk for sleep disturbances. Several environmental factors (outside noise, room temperature, and health worries) and emotional factors (depression, anxiety, and hostility) 
contribute to poor sleep quality among individuals of lower SES (Mezick et al., 2008).

\section{Other key important covariates (second research question)}

\section{Knowledge}

Knowledge and awareness of healthy sleep, including significance of sleep and the required amount of sleeping hours per night, shape the perception an individual may have regarding his/her sleep behavior. Studies that examined the role of knowledge in predicting sleep quality are varied, ranging from no or weak associations to strong associations. For example, in a cross-sectional study of sleep quality among university students, Suen, Tam, and Hon (2010) stated that knowledge has a non-significant effect on sleep quality, and claimed that knowledge in regard to proper sleep habits/behavior does not necessarily impact sleep quality. However, in an examination of the association between sleep behavior awareness and sleep quality among college students, Brown, Buboltz, and Soper (2002) found a weak association between knowledge of sleep behavior and good sleep quality. On the other hand, in a community population of adults who have been identified as poor sleepers, sleep knowledge was found to be a significant predictor of sleep behavior and better sleep quality (Gallasch \& Gradisar, 2007). In a motivational school-based intervention for adolescent sleep problems, Cain, Gradisar, and Moseley (2011) stated that as a result of sleep education, students became more motivated to regulate their out-of-bed times, and increase their average total sleep time to 9 hours. In another educational program conducted by Azevedo et al. (2008) among high school and university students, some aspects of sleep knowledge such as nap frequency and bedtime schedule were promoted due to sleep educational programs. Subsequently, students' sleep behavior such as taking a nap and regulating bed time improved.

\section{Sleep related factors}

Acccording to Harvard Medical School (2007), sleep quality and quantity can be 
influenced by various factors divided into internal and external factors. Internal factors include individual variation and genetics of sleep. However, the current study includes only sleep-related external factors. Sleep-related external factors are enormous and may include a broad continuum of elements that may directly or indirectly affect sleep. Therefore, the focus of this study will be on the most significant and common factors (the bedroom environment and drink intake before sleep [caffeinated]). In general, these factors have been shown to be the most influential to poor sleep quality (Harvard Medical School, 2007).

\section{Bedroom environment}

The bedroom environment can significantly impact the quality and duration of sleep. It is recommended to sleep in a room that is clean, neat, comfortable, cool, dark, quiet, and free from disruptions (Harvard Medical School, 2007; NSF, 2015). Factors related to the bedroom environment include bed and bedding, noise, room temperature, and light. According to the NSF (2015) and Ensor, Oexman, Scott, Carrier, and Davis (2008) the bed, mattress, and pillows also contribute to a good night sleep and can improve some aspects of sleep quality. For example, Jacobson, Boolani, and Smith (2009) compared sleep quality and stress-related symptoms between older beds ( $\geq 5$ years) and new bedding systems (new, medium-firm beds) in a convenience sample of healthy working adults. Results indicated significant improvements between pre and post test mean values in sleep quality and efficiency suggesting new bedding systems increased sleep quality.

Environmental noise, including bedroom noise, is considered one of the main factors that contribute to poor sleep quality (Brown et al., 2002; Muzet, 2007). Fyhri and Aasvang (2010) reported significant relationships between noise annoyance at night and sleeping problems in their investigation of the relationships among long-term noise exposure, annoyance, sleeping problems and subjective health complaints. In addition, Basner, Müller, and Elmenhorst (2011) 
found single and combined effects of road, rail, and air traffic noise on both objective and subjective assessments of sleep quality. Night-time exposure to traffic noise or low frequency noise has been found to be associated with subjective reports of lower sleep quality (Waye, Clow, Edwards, Hucklebridge, \& Rylander, 2003). Gellis and Lichstein (2009) have found in their comparison of sleep hygiene practices (sleep behavior) between good and poor sleepers that, excessive noise and uncomfortable night-time temperature have been reported by poor sleepers.

The effect of the thermal environment is linked to the thermoregulation, which affects the sleep regulating mechanism. Heat exposure can affect sleep stages by decreasing REM and slow wave sleep and then increased wakefulness while exposure to cold does not impact sleep, and that may be related to the use of clothes, bedding and blankets (Okamoto-Mizuno \& Mizuno, 2012). In fact, body temperature rhythm has been associated with different types of insomnia symptoms (Lack, Gradisar, Van Someren, Wright, \& Lushington, 2008). Light (both natural and artificial), particularly the exposure to room light, affects sleep directly by suppressing the production of the melatonin hormone that helps to induce sleepiness and regulates the sleep/wake cycle (Gooley et al., 2011; Schlangen, 2014). It has been reported that the circadian cycle is enhanced by the daytime light, which also makes the rhythm earlier (lead to early sleep). However, evening light delay the sleep/wake rhythm (lead to late sleep) (Schlangen, 2014).

\section{Drinks}

Drinks, particularly caffeinated drinks, energy drinks, and alcohol have been linked to sleep quality and duration. Caffeine increases sleep latency and alertness, and decreases sleepiness, which in turn affect sleep duration (Hershner \& Chervin, 2014). Daytime sleepiness also associated with increased consumption of caffeinated drinks such (Tran et al., 2014). Consumption of energy drinks is also associated with poor sleep quality (Sanchez et al., 2013). Malinauskas, Aeby, Overton, Carpenter-Aeby, and Barber-Heidal (2007) reported that about 
$51 \%$ of their sample of 253 college students consume more than one energy drink per month contributing to their poor sleep. Alcohol use, specifically binge drinking (at least 4-5 drinks in a row), has been found to have a significant positive association with sleep problems in both adolescents and adults; as binge drinking increases, more sleep problems occur (Popovici \& French, 2013). Consumption of alcohol is linked to different aspects of sleep quality, sleep duration, and bedtime delay (Chaput et al., 2012; Kenney et al., 2012; Singleton \& Wolfson, 2009). Lund et al. (2010) found that alcohol consumption is high among poor sleepers to induce and regulate their sleep. However, alcohol has a shortening effect on sleep latency, but then causes disrupted sleep (fragmented) in later sleep stages, which may lead to both poor sleep quality and short sleep duration (Hershner \& Chervin, 2014). In addition, students who frequently drink alcohol have sleep problems including later bedtimes especially on the weekend, less nighttime sleep, and daytime sleepiness (Ehlers et al., 2010; Singleton \& Wolfson, 2009). Galambos et al. (2009) suggest a decrease in both sleep quality and quantity (duration) occur after alcohol usage. Conversely, Chaput et al. (2012) and Kenney et al. (2012) reported that poor sleep quality and/or sleeping less than 6 hours per day lead to greater alcohol intake.

\section{HBM, Behavioral intention and Sleep HBM Perception Constructs and sleep}

HBM constructs have a combined and single influence on the likelihood of taking a recommended health action. High perceived threat, high benefits and low barriers together can lead to a high probability of taking the action (Sutton, 2002). Studies show that perception constructs were powerful and consistent significant predictors of behavior. Perceived susceptibility was a stronger predictor in preventive health behavior while perceived severity was a less powerful but consistent predictor (Carpenter, 2010; Glanz et al., 2008; Sutton, 2002). The HBM was used by Olsen, Smith, Oei, and Douglas (2008) in 77 patients newly diagnosed with 
obstructive sleep apnea to test the contribution of the constructs in the prediction of continuous positive airway pressure (CPAP) adherence. Results indicate that high outcome expectancies (i.e. perceived benefits) with treatment clarify the greatest proportion of adherence. Olsen, et al. (2008) concluded that perception of risk and outcome expectancies with treatment are important early predictors of initiating and continued use of continuous positive airway pressure (CPAP).

Among college students, Knowlden and Sharma (2014) used the HBM to measure and predict sleep behavior (or adequate sleep behavior, defined as receiving 7-8 continuous hours of sleep in 24 hour period) of employed students $(\mathrm{N}=188)$. They reported that $34 \%$ of the variance in sleep behavior was explained by the model. The most significant predictors were perceived severity, perceived barriers, cues to action, and self-efficacy. Individually, $22 \%$ of the variance in sleep behavior was (of the total $34 \%$ explained by the model) explained by perceived severity and $15 \%$ by perceived barriers.

\section{Behavioral intention and sleep}

Attitudes about sleep behavior, subjective norms about sleep behavior, and intention of performing healthy sleep behaviors have been found to have significant effects on sleep quality in adolescents (Nursalam, Apriani, Has, and Efendi, 2014). Sleep behavior (poor or healthy sleep) in adults, and among college students in particular, have been found to be best described by measuring the TPB constructs particularly, attitude, subjective norm and behavioral intention (Knowlden, 2011; Knowlden \& Sharma, 2012; Linden, 2011; Stanko, 2013). Knowlden (2011) was interested in operationalizing the constructs of TPB to predict sleep intentions and behaviors of undergraduate college students $(\mathrm{N}=197)$. Results suggested that as a collective prediction role, TPB accounted for $36.2 \%$ of the variance in the behavioral intention to obtain adequate sleep. In particular, subjective norm $(r=0.321, \mathrm{p}<0.001)$ and attitude $(r=0.357, \mathrm{p}<0.001)$ were 
significantly (moderate positive) correlated with behavioral intention. Behavioral intention was significantly (moderate positive) correlated with sleep behavior $(r=0.434, \mathrm{p}<0.001)$.

Knowlden and Sharma (2012) assessed the prediction role of the TPB of sleep intentions and behaviors among undergraduate minority students. Their results suggested that TPB accounted for $35.8 \%$ of the variance in the behavioral intention to obtain adequate sleep and $16 \%$ of the variance in sleep behavior was explained by behavioral intention. Finally, Stanko (2013) found in an investigation of sleep behavior among college students using the TPB framework that attitude and perceived norm are significant predictors of students' intentions to engage in sleep related behavior including intention to obtain 7-8 hours of sleep each night. Thus, the TPB is a useful framework in prediction sleep intentions and behaviors among college students (Knowlden \& Sharma, 2012).

\section{Sleep and HRQOL}

Sleep-related problems may affect or involve all aspects of life, which shape our quality of life (QOL). Both sleep quality and sleep duration have been linked to different aspects of quality of life. Preišegolavičiūte, Leskauskas, and Adomaitienè (2010) reported a significant association between quality of sleep and subjective QOL. According to Lopes, Esteves, Bittencourt, Tufik, and Mello (2008), excessive sleepiness and altered circadian rhythm may negatively affect ability to learn, employment and interpersonal relations, and may directly worsen quality of life. Lopes et al. (2008) also reported that patients with sleep apnea disorders have low QOL in all aspects including general health.

Health is one of the most important aspects of QOL affected by sleep behavior; and HRQOL refers to the physical and mental health aspects of QOL. In an examination of the crosssectional and longitudinal relationship between habitual sleep duration and HRQOL in older adults, Faubel et al. (2009) found that extreme sleep duration [short and long ( $\leq 5$ or $\geq 10 \mathrm{~h})$ ] was 
associated with worse scores on the Short Form (SF)-36 physical and mental scales, and with a worse score in the role-physical scale. Accordingly, it has been concluded that extreme sleep duration is an indicator of worse HRQOL. Herrick (2010) documented significant changes in HRQOL, including poor physical or mental health, and restricted activity, from low to medium and medium to high associated with levels of sleep-loss. Thus, it has been asserted that poor quality of life and sleep-loss are highly correlated. Consistently, poor sleep and/or sleep disturbances are associated with a variety of adverse health behaviors and impairment in several HRQOL domains including poor general health, frequent physical distress, frequent mental distress, activity limitations, depressive symptoms, anxiety, and pain (Andruškienè, Varoneckas, Martinkėnas, \& Grabauskas, 2008; Lee et al., 2009; Strine \& Chapman, 2005). However, JeanLouis, Kripke, and Ancoli-Israel (2000) reported an association between the quality of wellbeing and sleep satisfaction (perceived sleep satisfaction).

\section{Theoretical Rational}

There is a lack of theory-based description of sleep behavior among college students. To the best of the author's knowledge, the only researches that developed theoretical frameworks using the HBM and the TPB to measure and predict sleep behavior among college students were by Knowlden and Sharma (2012), Knowlden and Sharma (2014), and Knowlden (2011). However, there are some limitations within these studies. First, HBM and TPB have been used independently; thus, there is no evidence of using both theories as an integrated framework (integrating constructs from both theories) to predict sleep behavior as proposed in the current study. Integrating constructs from both theories as one framework allows for a comprehensive explanation of sleep behavior as suggested by the review of literature. Second, Knowlden and Sharma (2012) and Knowlden and Sharma (2014) tested the theoretical constructs in relation to 
adequate sleep behavior as defined as obtaining 7-8 hours of sleep within the previous 24 hours. Assessing sleep in just one day is insufficient and may not reflect the actual sleep behavior. The current study proposes to extend the period of assessment to 30 days. In addition, Knowlden and Sharma only assessed sleep duration which also may give an incomplete picture of sleep quality (good or poor). Hence, the current study will assess both sleep duration and sleep quality for a more inclusive evaluation of overall sleep quality. Finally, no study has reported the inclusion of demographic variables in the analysis of the model, except for the inclusion of gender in the application of the TPB by Knowlden (2011).

\section{Method}

\section{Study Design, Setting and Population}

This study aims to describe college students' sleep quality using behavioral constructs from both the TPB and HBM as an integrated theoretical framework among a sample of undergraduate West Virginia University students. Specifically, this cross-sectional study evaluates the effectiveness of the HBM perception constructs and the TPB behavioral intention in predicting student's sleep quality. West Virginia University [WVU] is a public university located in Morgantown, WV. WVU is composed of 15 colleges offering 193 Bachelor's, Master's, Doctoral, and Professional degree programs (West Virginia University, 2015). In fall 2014, the main campus enrollment was 29,175 (22,563 undergraduate, 5,001 graduate, and 1,611 professional students). Of these, $52 \%$ were male, and $48 \%$ were female students; in addition, $51 \%$ of students were non-West Virginia residents (WVU, 2015).

\section{Sampling}

\section{Sample size and Power analysis}

The power and sample size estimations were based on both sampling precision and our primary objective using a two-sided, two-sample t-test. A stratified random sampling technique 
was conducted to secure a more potential representative sample. A sample of 363 students was determined to be the minimum number of participants recommended based on the actual number of undergraduate students enrolled in Fall 2014 (22,563 students), and the prevalence of poor sleep among college students $(60 \%)$ presented in the literature (with a margin of error of 0.05 and 95\% confidence interval) (Raosoft, a sample size calculator software at:

http://www.raosoft.com/samplesize.html, and the National Statistical Services website: http://www.nss.gov.au/nss/home.nsf/pages/Sample+size+calculator).

A two-sample t-test was used to assess the sleeping score between student's characteristics and used in the sample size and power analysis. A sample size of 363 students will have $80 \%$ power to detect the mean difference on sleeping scores by at least 0.5 standard deviations between two groups at a 0.05 significance level, assuming the proportion of a dichotomized variable is $10 \%$ and $90 \%$ (high and low) between two groups. More scenarios on proportions of a dichotomized variable are showed in the Table below.

Two-sample t-test effect size (Power: $80 \%, \alpha=0.05, N=363$ ), where SD is the standard deviation of the sleeping score.

\begin{tabular}{|l|c|c|c|c|c|}
\hline $\begin{array}{l}\text { Proportions of } \\
\text { independent } \\
\text { variables (high vs } \\
\text { low) }\end{array}$ & $\mathbf{5 0 \%}$ vs 50\% & $\mathbf{4 0 \%}$ vs 60\% & $\mathbf{3 0 \%}$ vs 70\% & $\mathbf{2 0 \%}$ vs 80\% & $\mathbf{1 0 \%}$ vs $\mathbf{9 0 \%}$ \\
\hline Detected differences & $0.30 \mathrm{SD}$ & $0.31 \mathrm{SD}$ & $0.33 \mathrm{SD}$ & $0.37 \mathrm{SD}$ & $0.5 \mathrm{SD}$ \\
\hline
\end{tabular}

Accounting for drop-off and lower response rate, our initial sampling frame included 2200 students to get the minimum 363 students.

\section{Participants' recruitment}

As inclusion criteria, the sample involved undergraduate college students between the ages of 18 and 24 currently enrolled as full time students. Students were accessed through the 
Office of the University Registrar. First, we requested student data including their e-mail addresses and student identification numbers (ID); these data are anonymous. Then, a stratified random sample was performed as follows. The body of undergraduate students $(\mathrm{N}=13,086$ students, in 2016) was divided into distinct subgroups based on their year of enrollment: freshmen $(n=4,726 ; 36.1 \%)$, sophomores $(n=3,482 ; 26.6 \%)$, juniors $(n=2,685 ; 20.5 \%)$, and seniors $(n=2,193 ; 16.8 \%)$. Next, a random sample from each stratum was taken based on the ratio of the subgroup's size to the total number of undergraduate students. Accordingly, our initial sample frame consisted of 2200 students: $36.1 \%$ freshmen ( $\mathrm{n}=795), 26.6 \%$ sophomores $(\mathrm{n}=585), 20.5 \%$ juniors $(\mathrm{n}=451)$, and $16.8 \%$ seniors $(\mathrm{n}=369)$; as potential participants.

Then, potential participants $(\mathrm{N}=2200)$ were invited electronically to participate in the study via e-mail. An email pre-notification message was sent to each selected participant (see Appendix E for all correspondence with students) about the forthcoming survey. The invitation contained information about him/her being selected into the study, the study's purpose and importance, contact information for the principal investigator, and that the information they provide will be anonymous. The pre-notification email explained that if they participated in the study, they would have a chance to win one of 50 Panera gift cards ( $\$ 10$ each). Five days after the pre-notification phase, another e mail requesting participation in the survey was sent to the selected participants. At the bottom of the email, a link to the questionnaire was provided through a pre-designed WVU-based website (for student convenience and legitimacy reasons). The link directed participants to the informed consent; by clicking "agree" on this page, students indicated their willingness to participate, and then moved to the subsequent electronic survey.

Students who refused to participate had the option to click "disagree", and were directed to a thank you message, and information on how to later participate if they changed their decision. A 
total of three follow up e-mail reminders (days 8,15 , and 21) were sent to those who had not responded or who choose not to participate asking them to reconsider their participation. The survey was developed using Qualtrics software (2016), and was pilot tested for accuracy in terms of skip patterns, etc. The survey was active for 30 days (from September 22, 2016 to October 22, 2016). At the end of October incentives were distributed among 50 winners (who were randomly selected) based on their preferences (electronically, traditional mailing or through the University Registrar Office). We received 494 completed surveys, yielding a random sample of 494 undergraduate students. Response rate was $22.5 \%$, which is greater than the reported $20 \%$ response rate of online surveys (Sax et al., 2003). The study was approved by the Institutional Review Board (IRB) at West Virginia University (WVU) (WVU IRB's Research Protocol: 1603066826) (see approval letter Appendix F).

\section{The Survey (Instruments)}

(see Appendix D)

The survey is a self-reported questionnaire that incorporated several scales to measure Health-related quality of life (HRQOL Modules), Sleep quality (PSQI), TPB constructs instrument, and the HBM constructs instrument; as well as questions related to the study covariates.

\section{Health related quality of life: $H R Q O L-14$ "Healthy Days Measure"}

HRQOL was measured using the HRQOL-14 "Healthy Days Measure" (CDC, 2011).

The HRQOL-14 "Healthy Days Measure" is an integrated set of broad questions about recent perceived health status and activity limitation during the past 30 days. The module or scale includes the core module of Healthy Days (CDC HRQOL-4), and ten additional questions about HRQOL: Activity Limitations Module and Healthy Days Symptoms Module. The expanded HRQOL-14 questions measure the burden of both short-term and persistent physical and mental 
health problems (CDC, 2000). The core module includes four questions that measure in general the health status, physical health, mental health, and the usual activities. Activity Limitations Module and the Healthy Days Symptoms Module consist of five questions each asks about recent pain, depression, anxiety, sleeplessness, vitality, and the cause, duration, and severity of a current activity limitation an individual may have in his or her life. Questions related to sleeplessness were intentionally omitted, because they were assessed by the Pittsburgh Sleep Quality Index [PSQI].

The instrument had varied response options. The core module had a 5-point Likert scale ranging from excellent to poor for question 1; and number of days' responses for the rest of the questions. The activity limitation module had yes/no responses for questions 1, 3, 4, and 5; while question 2 asked for a specific problem to be identified from a list of different health issues. The last module of healthy days symptoms asked about number of days. The HRQOL questions were scored using a summary "unhealthy days" index. Unhealthy days were an estimate of the overall number of days during the previous 30 days, when the respondent felt that either his or her physical or mental health was not good. It was computed by adding a respondent's physically and mentally unhealthy days, with a range of 0-30 days for each person. The "Healthy days" index can be used as a complementary form of the "unhealthy days"; it is calculated by subtracting a respondent's unhealthy days from 30 days. This index estimates the minimum number of recent days when a respondent had good or better health and provided a positive measure of HRQOL (CDC, 2011; Moriarty, Zack, \& Kobau, 2003).

Validity and reliability

Several studies have indicated that the CDC HRQOL- 14 has adequate construct validity (CDC, 2000; Moriarty et al., 2003). In addition, in a general statewide population, the single 
global measure in the CDC HRQOL-14 had acceptable criterion validity with multiple-item SF36 scales (CDC, 2000) in a manner consistent with expectations. The SF-36 is generally considered the "gold standard" in HRQOL measurement. Moreover, the HRQOL-14 has displayed estimates of acceptable test-retest reliability and construct validity in a representative telephone sample of Missouri adults (CDC, 2000; Moriarty et al., 2003). In addition, Andresen, Catlin, Wyrwich, and Jackson-Thompson (2003) reported an excellent retest reliability (0.75 or higher) for Self-Reported Health and Healthy Days measures (the core module).

\section{Sleep Quality Scale (Pittsburgh Sleep Quality Index [PSQI])}

Sleep can be assessed or measured subjectively or objectively using different instruments and scales. Subjectively, sleep can be measured by using self-report methods including: sleep diary, sleep log, and sleep questionnaire (sleep scales). These methods assess both sleep quantity and quality as experienced by the individual. Subjective measures are easily administered, inexpensive, and applicable to large population (Yi, Shin, \& Shin, 2006). This study subjectively assessed sleep quality using the PSQI. The PSQI is a self-rated questionnaire that assesses sleep and disturbances over a 30 day time interval (Buysse et al., 1989). The scale consists of 19 individual items generating seven components: subjective sleep quality, sleep latency, sleep duration, habitual sleep efficiency, sleep disturbances, use of sleeping medication, and daytime dysfunction (Buysse et al., 1989). There are five additional questions rated by the partner or roommate. These questions are used for clinical information only, and are not tabulated in the scoring of the PSQI (Buysse et al., 1989).

The scale contains different response options. Questions 1-4 ask for a specific number, such as hours of sleep per night, bed time, and getting up time. Questions 5, 7, and 8 use a frequency scale (not during the past month, less than ones a week, once or twice a week, or three or more times a week). Questions 6 and 9 have two different four- point Likert-scales. The Likert 
scale for question 6 has the following options: very good, fairly good, fairly bad, and very bad. Question 9 Likert scale has the following options: no problem at all, only a very slight problem, somewhat of a problem, and a very big problem. The 19 items of the scale are grouped into 7 components scores. Each component weighted equally on a 0-3 scale. Then, the 7 components scores are summed to produce a global PSQI score ranging from 0-21, with higher scores indicate worse sleep quality. Finally, the entire scale requires 5-10 minutes for participants to complete, and 5 minutes for scoring (Buysse et al., 1989).

\section{Reliability and validity}

Internal consistency and test-retest were used to test the reliability of the PSQI. The scale has demonstrated high internal consistency estimates with Cronbach's alpha coefficients of 0.83 . In addition, it has been documented to be stable over time (Buysse et al., 1989; Yi et al., 2006). T-test and Pearson product-moment correlations of the scale showed no significant differences between time 1 and time 2 (an average of 28.2 days between time 1 and time 2) (Buysse et al., 1989). In addition, the instrument has also displayed an adequate validity. Concurrent validity has been tested to assess the degree to which the index detects differences between groups with and without sleep problems. A global PSQI score $>5$ give in a diagnostic sensitivity of $89 \%$ and sensitivity of $85 \%$ (Kappa $=0.75, \mathrm{p}<0.001)$ in distinguishing good and poor sleepers (Buysse et al., 1989).

The Theory of Planned Behavior Instrument (Behavioral Intention)

This study utilized a psychometrically robust TPB based instrument developed by

Knowlden (2011). The instrument measured the proximal TPB constructs. Behavioral intention is measured with 3 items using 7- point semantic differential scales; the responses included, "I intend", "I will try", and "I plan", to measure the intention of the participants to engage in good 
sleep quality behavior. Scores for behavioral intention range from 3 to 21; higher scores reflected a greater intention to perform the target behavior (Knowlden, 2011; Knowlden \& Sharma, 2012).

\section{Validity and reliability}

The instrument has been found to display satisfactory estimates of construct validity, test retest reliability and internal consistency. In addition, face and content validity was validated by a panel of six experts (Knowlden, 2011).

\section{The Health Belief Model (HBM) Instrument}

This study utilized the HBM scales (a 52-item instrument) developed by Knowlden and Sharma (2014) to measure and predict the sleep behavior of employed college students who operated motor vehicles and were not diagnosed with a sleep disorder; with some modifications to fit the purpose of this study. The following are the scales:

\section{Perceived susceptibility scale:}

The perceived susceptibility construct stem statement, "if you were unable to sleep 7 to 8 continuous hours each day, how likely would you be to. . " is preceded by six 5-point semantic differential scales. Sample scale items included, "eventually develop a sleep related disorder (e.g. insomnia)," "engage in one or more negative health behaviors (e.g. substance use, physical inactivity" and "eventually develop a negative health outcome (e.g. overweight or obese, chronic diseases)," and "unintentionally fall asleep while driving a motor vehicle." Scale endpoints included, "not at all likely_extremely likely." Perceived susceptibility construct has an overall score ranges from 6 to 30, with higher score representing greater perceived susceptibility to the consequences of poor sleep.

\section{Perceived severity scale:}

The stem statement for the perceived severity construct is: "if you were unable to sleep 7 to 8 continuous hours each day, how likely would you be to ..." preceded by five 5-point 
semantic differential scales. The scale items included: "maintain good physical health," "remain alert while driving," and "control your appetite." Scale endpoints included, "no effect—major effect." This construct has a score range of 5 to 25 , with higher scores representing greater perceived severity to the consequences of poor sleep.

\section{Perceived benefits scale:}

The stem statement of the perceived benefits construct is: "how beneficial to you is sleeping 7 to 8 continuous hours each day for ..." it is followed by four 5-point semantic differential scales. Items included: "your energy levels," "your school productivity,” and "your general health and quality of life." Scale endpoints included, "not at all beneficial—extremely beneficial." The construct has a score range of 4 to 20 , with higher scores representing greater perceived benefits of achieving good sleep quality.

\section{Perceived barriers scale:}

The perceived barriers construct stem statement is: "how likely are you to not get 7 to 8 continuous hours of sleep each day because ..." Followed by four 5-point semantic differential scales. Sample items included: "you feel pressured for time," "you feel behind on your school work," and "it is easier to sacrifice sleep during schooldays and make up for lost sleep during the

weekend." Scale endpoints included, "not at all beneficial—extremely beneficial." The construct has a score range of 4 to 20 , with higher scores representing greater perceived barriers for achieving good sleep quality.

\section{Reliability and validity:}

The instrument items were refined over two rounds by a panel of six experts. The review process reported unanimous face and content validity. Readability of the instrument was tested with the Flesh Reading Ease Test and Flesh-Kincaid Grade Level Test. Respective readability 
test scores were 64.7- and 6.7-grade levels and considered adequate for college populations (Knowlden \& Sharma, 2014).

\section{Questions related to the external variables}

Questions on the external variables including demographic; sleep-related environment are included in the PSQI and theoretical instruments. In addition, questions to assess sleep knowledge were selected from the Views On Sleep Scale (VOSS) (Dolan, 2013). The scale contains 25 items that measure various beliefs, attitudes, expectations, and attributions about sleep and insomnia. The overall scale has good internal reliability (Cronbach's $\alpha=0.792$ ) (Dolan, 2013). Higher scores indicate high knowledge regarding sleep and its significance.

\section{Data Analyses}

Descriptive statistics and exploratory data analysis including frequency, mean, standard deviation, and percentage were performed to summarize students' demographic characteristics. Categorical data were described using contingency tables. Continuously scaled measures were summarized also with descriptive statistical measures (i.e., mean \pm s.d.). This study has two outcomes: primary (sleep quality) and secondary HRQOL (Unhealthy days). Prediction and correlation analysis are the main statistical interests of this study, based on the primary and secondary research questions. In the primary research questions, we are specifically interested in assessing whether the HBM perception constructs and behavioral intention have roles in describing and prediction sleep quality among WVU college students. Therefore, we conducted a structural equation modeling (SEM) using AMOS (controlling for gender, race and year of enrollment as their potential influences on the outcomes). SEM allowed us to explicitly model both direct and indirect effects using both measured and latent variables. Control variables for this model were coded as: gender is $0 / 1$ with girls $=1$, enrollment is $1-4$ with seniors $=4$, and 
race is white $=1$, other $=0$. Additionally, we assessed the relationship between all the variables in the hypothesized model including HRQOL.

To answer the secondary research question, we conducted a multivariate linear regression (using SAS 9.4 for Windows), to assess the link between sleep quality, particularly poor sleep and unhealthy days (HRQOL), controlling for a list of important covariates for their potential influences on both outcomes particularly sleep quality including: Demographics (race, gender, age, Job, Marital Status, and Year of Enrollment); Substance use (cigarette, alcohol and marijuana use); NMUPD (Antidepressant painkillers Sedatives Stimulants); being diagnosed for [ADHD (Attention Deficit Hyperactivity Disorder), insomnia, sleep disorders, and depression]; the overall perceived stress level; weight and Exercises (BMI, intensity exercise, Vigorous exercise, strength Exercises, exercise to lose weight, diet to lose weight, and pills to lose weight), Sleep-related (use of sleep medication, sleeping on an uncomfortable bed, sleeping in an uncomfortable bedroom); and the everyday use of caffeinated drinks (coffee, tea, soda and energy drink); and excluding gender when modeling by gender. All statistical tests where twosided and $\mathrm{P}<0.05$ was considered statistically significant.

\section{SEM Testing}

A series of six measurement models were evaluated to get the best fit model using confirmatory factor analysis. Confirmatory factor analysis was used to test the fit of the hypothesized factor structure to the covariance matrix of the observed variables. In the construction of all latent variables, we used confirmatory factor analysis from the beginning, as the latent variables already made clear what indicators we should be seeking. During the model building, perceived barriers was dropped from the model because of poorer model fit. This is not uncommon in HBM research where barriers have often been found to be the least important of 
the HBM indicators (Glanz et al., 2008). The final model included three indicator variables for the latent variable Health Belief Model "HBM", three predicted variables [Behavioral Intention (Theory of Planned Behavior construct), Global Sleep Quality Score, and Unhealthy days] (observed), and the control variables (including gender, race and year of enrollment at the University). The fit of the final structural model was acceptable $(\chi 2=28.543, d f=13, P<0.05$; CFI $[$ comparative fit index $]=0.97$, and RMSEA [root mean square error of approximation $]=$ 0.065). Models are considered a good fit if the CFI is .90 or above, and very good if it reaches .95 , so this model fits very well to the data by CFI standards. Furthermore, RMSEA is a badness of fit measure and is considered acceptable if it is .08 or below and very good if it is .05 or below. Our model is in the "good" rank with RMSEA (Hu \& Bentler,1999).

\section{Model's variables}

The final developed model includes: Unhealthy days (measure of HRQOL) the secondary outcome as a predicted variable, Sleep Quality (GSQS), the primary outcome as a predicted variable, the HBM (predictor) and has three indicators: [Susceptibility (perceived susceptibility), Severity (perceived severity), and Benefits (perceived benefits)]. Intention (measure of TPB's behavioral intention) as both predictor and predicted/ variable. In addition, three demographic variables were included in the model as control variables (gender, race and year of enrollment). 
Results

\section{Sample characteristics:}

\section{Demographics}

Table 1 and Figures $1 \& 2$ (Appendix C) present sample characteristics $(\mathrm{N}=494)$. Participants included 211 females (61.3\%) and 133 males $(38.7 \%)$ students (Mean age=20, $\mathrm{SD}=5.7)$. Students were primarily White $(87 \%)$. However, the lowest presentation of ethnicity has been recorded for American Indian, Alaskan Native, or Native Hawaiian (0.6\%). Around $42 \%$ of students were freshman, and almost $60 \%$ were not employed (Table 1a). The majority of students (58\%) reported using alcohol in the past 30 days, and approximately $8.2 \%$ of students reported the nonmedical use of stimulants. Gender differences reveal that females have higher usage rates than males for all the classes of the NMUPD. The highest difference was among painkillers users, $80 \%$ of them were females compared to $20 \%$ males (Table $2 \mathrm{c}$ ). In addition, most students (97.4\%) reported stress level of average and above; and approximately 14\% reported being diagnosed with depression (Table 2c) in the past 12 months. Females who have reported stress, depression and have been diagnosed and/or treated for ADHD, insomnia and sleep-related disorders were higher than males, almost double. Moreover, around $23 \%$ of students were classified as overweight. Exercise was the most common way of losing weight reported by student $(44 \%)$ in the past 30 days (Table $2 \mathrm{c})$.

\section{Sleep quality, HRQOL and the behavioral constructs}

Table 1b, shows the distribution of sleep-related factors including sleep knowledge, Bedroom environment, sleep medication and the everyday use of caffeinated drinks. The average sleep knowledge score was 20.5 (range 5-40 with higher score indicating higher sleep knowledge). During the past 30 days, around $22 \%$ of students reported sleeping in an uncomfortable bedroom (hot/cold, noise, light), while only $4.7 \%$ reported sleeping in an 
uncomfortable bed. Among the caffeinated drinks, soda was the most common drinks reported by about $54 \%$ of students. Coffee was the popular caffeinated drinks among females (67.6\%), while, soda was common among males (50.3\%) (Table 1b).

Table $2 \mathrm{~b}$, presents sleep quality and HRQOL characteristics among students. The average sleeping hours per night during past month was 6.7 hours. The average sleep quality scores recorded based on the PSQI was $6.2(\mathrm{SD}=4.2)$, higher score indicates worse sleep quality. Based on this measure of sleep, poor sleep quality was found to be prevalent among students, as $69 \%$ has been classified as poor sleeper with $67 \%$ being female. The average number of unhealthy days (in the past 30 days) was $13(\mathrm{SD}=10)$ days. Finally, most students (57.3\%) rated their overall health as very good, and around 17\% reported fair overall health. Among students who reported fair health, almost $68 \%$ were females.

The HBM perception constructs and the behavioral intention have different scores ranges (susceptibility:4-28, severity:5-25, benefits: 4-20, and behavioral intention:3-21), higher score indicates high perception, and high behavioral intention (which is positive). The average scores have been reported as (Table 2a):18.8 ( $\mathrm{SD}=5.50)$ for perceived susceptibility; $17.8(\mathrm{SD}=4.9)$ for perceived severity; $17.2(\mathrm{SD}=3.8)$ for perceived benefits; and $16.0(\mathrm{SD}=5.3)$ for behavioral intention.

\section{Structural Equation Modelling (SEM)}

The results of the SEM presented in Figure (1) and Table (3a). Figure (1), illustrates the hypothesized relationships between variables in this study. Table (3a1-2) represents the context of the hypothesized relationships of our model and the standardized $(\beta)$ and unstandardized regression weights from the structural equation model, along with standard errors and critical ratios for statistical significance. As shown in the figure, the HBM is related to the TPB intention 
scores (Behavioral Intention), GSQS (sleep quality), and to unhealthy days. Intention score was related to sleep quality. And finally, GSQS (sleep quality) was related to unhealthy days (HRQOL). In other words, sleep quality was predicted by behavioral intention (TPB) and the HBM. Unhealthy days was predicted by sleep quality, and the HBM. Behavioral intention was predicted by the HBM. Among the control variables, only gender was related to HBM and sleep quality. Around $20 \%$ of the variance in sleep quality was explained by the HBM, behavioral intention and the control variables (Gender). In addition, roughly, $31 \%$ of the variance in unhealthy days was explained by sleep quality, behavioral intention, the HBM, and the control variables.

The HBM was created with latent loadings from perceived severity $(\beta=.91)$, perceived susceptibility $(\beta=.74)$, and perceived benefits $(\beta=.62),(\mathrm{P}<.05)]$. HBM had a negative indirect relationship (mediated through behavioral intention) with sleep quality (standardized $\beta=-.12$, $\mathrm{p}<.01$ ), which suggests that if HBM increased by one standard deviation unit, sleep quality would be expected to decline by .12 standard deviation units. HBM also had a significant positive and direct association with behavioral intention (standardized $\beta=.29, \mathrm{p}<.01$ ), for each one standard deviation unit increases in HBM, behavioral intention increased by .29 standard deviation units. The intention score which reflects the TPB behavioral intention, has a strong significant direct negative association with sleep quality (standardized $\beta=-.40, \mathrm{p}<.01$ ), indicating that when the behavioral intention score increases by one standard deviation unit, the sleep quality scores decreases by .40 standard deviation units. Based on these relationships between HBM, behavioral intention and sleep quality, HBM contributes strongly to behavioral intention (.29), and behavioral intention is very strongly related to sleep quality (-.40); therefore, the impact of HBM on sleep quality is likely to be related to its impact on behavioral intention. 
The direct relationship between HBM and sleep quality is positive (standardized $\beta=.152$, $\mathrm{p}<.05$ ), and it is only significant in the absence of the negative mediated effects reported earlier (standardized $\beta=-.12$ ).

Unhealthy days was predicted by the HBM and sleep quality. The HBM has a direct positive relationship with unhealthy days (standardized $\beta=.19, \mathrm{p}<.01$ ), suggesting that for each standard deviation unit increase in the HBM scores, unhealthy days increased by a standard deviation of .19 days. Sleep quality was a very strong predictor of unhealthy days with a standardized $\beta=.46(\mathrm{p}<.01)$, as for each standard deviation unit increases in sleep quality, unhealthy days increase by .46 standard deviation units. Interestingly, we found that behavioral intention is indirectly related to unhealthy days through sleep quality. When the behavioral intention scores increase by one standard deviation unit, unhealthy days decrease by .19 standard deviation units. This holds despite a non-significant total effect between intention and unhealthy days. Finally, among the control variables only gender was a significant factor in the hypothesized relationship, and has a direct association with both $\operatorname{HBM}(\beta=.35, \mathrm{p}<.01)$, and with sleep quality $(\beta=.28, \mathrm{p}<.01)$.

\section{Multivariate Linear Regression Model}

Additionally, a multivariate regression model was conducted to assess the relationship between sleep quality and unhealthy days, with the purpose of including a list of important demographic and behavioral covariates that we were unable to include in the SEM due to model fit issue. However, consistent with the SEM the regression results (Table 3b: Appendix C), revealed a significant overall effect of sleep quality on unhealthy days $(F(51,238)=4.70, p<$ 0001) with an $\mathrm{R}^{2}$ of 0.50 ; even after controlling for list of covariates. The model explained $50 \%$ of the variance in unhealthy days (with a large effect size $\mathrm{R}=0.71$ ). Students' unhealthy days 
increased by 0.82 day for each unit increase in sleep quality scores. When analyzing by gender we found the association is only significant among females; their unhealthy days is predicted to increase by 0.66 days for each unit increased in sleep quality scores.

\section{Discussion}

The primary purpose of this study was to use a theoretical framework to explain and or predict sleep quality and HRQOL of a sample of WVU undergraduate college students ( $N=494)$. Additionally, we were also interested in exploring the relationship between sleep quality and HRQOL as reflected by unhealthy days. This is the first known study to apply a theoretical model using the HBM (with three constructs: perceived susceptibility, perceived severity, and perceived benefits) along with behavioral intention from the TPB. We sought to extend previous work by estimating the prediction roles of the identified theoretical constructs using SEM; and by assessing the relationship between sleep quality and HRQOL in a multivariate regression model considering key demographics and behavioral covariates in both models.

Results revealed that poor sleep is prevalent among our sample of WVU undergraduate college students. First, the reported average sleeping hours per night during past month (6.7 hours), is below the recommended 7-8 hours of sleep per night (CDC, 2013; NIH, 2012a). The average sleep quality scores recorded based on the PSQI was $6.2(\mathrm{SD}=4.2)$, which indicates poor sleep quality, based on the cut off ( $\geq 5$ ) considered to be worse sleep quality (Buysse et al., 1989). Finally, based on this cut off measures, $69 \%$ of students were classified as poor quality sleepers. Correspondingly, the literature affirmed the prevalence of poor sleep among college students, with more than 60\% classified as poor quality sleepers (Tsai \& Li, 2005; Lund et al., 2010). Female students in our sample reported poorer sleep quality, supporting previous research suggesting females report poorer sleep quality than males (Tsai \& Li, 2005). 
Poor sleep among our sample may be explained by several sleep-related factors, including as an example; sleeping in an uncomfortable bedroom (during the past 30 days) which was reported by around $22 \%$ of students, and the prevalent of caffeinated drinks: soda (reported by $54 \%$ of student) and Coffee (reported by 53. \%). Bedroom environment (including noise, light, and temperature) and drink intake before sleep have been found to be the most influential to poor sleep (Brown et al., 2002; Harvard Medical School, 2007; Muzet, 2007). Moreover, caffeinated drinks, have been linked to poor sleep quality and duration, as caffeine increases sleep latency and alertness, and decreases sleepiness, which in turn affects sleep duration (Hershner \& Chervin, 2014). In relation to the prevalence of unhealthy days among students, the average of 13 days, which almost half of the month of having unhealthy days, may reflect poor HRQOL among students. The reported averages of HBM perception constructs and the TPB behavioral intention in general are high which shows that students have high perceived susceptibility and severity toward the consequences of poor sleep, high perceived benefits toward performing healthy sleep behavior, and have high intention to perform healthy sleep behavior.

Consistent with study expectations, the structural equation model (controlling for gender, race and year of enrollment), showed significant associations between the perception constructs of the HBM, behavioral intention, sleep quality, and unhealthy days. The HBM was strongly associated with behavioral intention, sleep quality, and unhealthy days. Behavioral intention was directly associated with sleep quality and indirectly (through sleep quality) connected to unhealthy days. And finally, there was a positive significant relationship between sleep quality and unhealthy days. Thus, sleep quality was predicted by behavioral intention (TPB) and the HBM; and unhealthy days was directly predicted by sleep quality and the HBM, and indirectly 
by behavioral intention. The HBM was created with latent loadings from perceived severity, perceived susceptibility, and perceived benefits. Perceived susceptibility refers to the possibility of getting one or more of the negatives outcomes associated with poor sleep quality; perceived severity is the perception of the significance, seriousness and consequences of getting one or more of the associated negative outcomes; and perceived benefits is the belief of the potential rewards of practicing healthy sleep. The behavioral intention refers to the intention to perform healthy sleep behavior (Knowlden, 2011).

The ability of the HBM in prediction sleep has been reported by Knowlden and Sharma (2014). They have used the HBM to measure and predict sleep behavior (refers to sleep duration: defined as receiving 7-8 continuous hours of sleep in 24 hour period) of employed college students. They reported that $34 \%$ of the variance in sleep behavior was explained by the model (that included all the constructs of the HBM). Perceived severity has been found consistently to be the most significant predictor of HBM (Carpenter, 2010; Glanz et al., 2008; Sutton, 2002; Knowlden and Sharma, 2014). In our study, the HBM (with only three constructs) shared with behavioral intention and gender, explained $20 \%$ of the variance in sleep quality (which is more inclusive than sleep duration, that included both sleep duration and subjective indices of sleep quality).

HBM constructs have a combined and single influence on the likelihood of taking a recommended health action (Sutton, 2002). High perceived threat (combination of perceived susceptibility and perceived severity), high benefits and low barriers together can lead to a high probability of taking the action (Sutton, 2002). Similarly, we found that HBM (perceived susceptibility, perceived severity, and perceived benefits) was strongly associated with behavioral intention (TPB). Behavioral intention is defined as the perceived likelihood of 
carrying out the behavior (Glanz et al., 2008). This association suggests that as HBM increased (high perceived susceptibility, high perceived severity, and high benefits), the likelihood and intention to perform healthy sleep behavior increased in the sample. However, perceived barriers construct was not included here because of poorer model fit. Commonly, perceived barriers known as the least important of the HBM indicators (Glanz et al., 2008).

In line with current studies, our results identified a negative association between behavioral intention and sleep quality. This result, signifying that getting good sleep quality is greatly associated with having high intention to perform healthy sleep behavior. Behavioral intention is considered as the most significant indicator of the TPB (Knowlden and Sharma, 2012). Knowlden and Sharma (2012) suggested a significant prediction role of the behavioral intention, as $16 \%$ of the variance in sleep behavior (adequate sleep duration) among undergraduate minority students was explained by behavioral intention. Knowlden (2011) also found a significant correlation between behavioral intention and sleep behavior (adequate sleep duration) among undergraduate college students. In addition, Nursalam, et al. (2014) reported a significant effect of the intention of sleep behavior on sleep quality in adolescent.

In our study, the association between the HBM perception and sleep quality was mediated by behavioral intention. The results reveal that; sleep quality scores are expected to decrease when HBM increased. This relationship in the present of a mediation effect, can be explained as: high HBM perception (susceptibility, severity and benefits) toward sleep behavior increases the intention to perform healthy sleep behavior, and then leads toward achieving good sleep quality. Hence, good sleep quality is the outcome of having high behavioral intention, which is significantly achieved by having high HBM perceptions toward sleep behavior. This finding is 
unique, to the best of author knowledge, there is no evidence of existing studies that have examined the association between HBM, behavioral intention and Sleep Quality.

Additionally, we have added unhealthy days (HRQOL) in our SEM as a secondary outcome, with the intention to assess the association between our variables in the model particularly sleep quality with unhealthy days. The results suggest that around $31 \%$ of the variance in unhealthy days was explained by sleep quality, behavioral intention, the HBM and the control variables. The HBM had a direct significant positive association with unhealthy days, suggesting that when HBM increases, unhealthy days increase. Which means having high perception toward sleep behavior increases unhealthy days. Although, this results is statistically significant, practically does not make sense and considered as nonsignificant. A possible explanation for this is that the HBM constructs were operationalized in terms of sleep quality aspects as. On the other hand, behavioral intention had a negative indirect association with unhealthy days. As having high intention to perform healthy sleep behavior leads to decreases in unhealthy days. This impact is mediated by sleep quality, as high behavioral intention associated with getting good sleep quality. Sleep quality, in turn, was positively associated with unhealthy days, as increases in sleep quality scores (worse sleep quality) associated with increasing in the number of unhealthy days. Finally, among the control variables, only gender was found to be significantly related to both HBM and sleep quality.

The multivariate linear regression results confirm the association between poor sleep quality and unhealthy days found in the SEM, even after controlling for several demographic and behavioral covariates. The results indicated that students' unhealthy days increased by 0.82 days for each unit increase in sleep quality scores, suggesting that poor sleep quality (reflected by increasing in sleep quality scores) is associated with having more days of unhealthy days (which 
also reflect poor physical and mental health). Among gender, the association was significant among female only. Females' unhealthy days would be increased because of having poor sleep quality. The base line distribution of poor sleep quality and unhealthy days in our results may have role in this relationship as females have poorer and more unhealthy days than males.

Results of both the SEM and the regression models concurs with the current literature asserting a significant association between sleep quality and subjective HRQOL (Andruškienė, et al, 2008; Faubel et al., 2009; Herrick, 2010; Lee et al., 2009; Lopes, et al., 2008; Martinkėnas, \& Grabauskas, 2008; Preišegolavičiūtè, et al., 2010; Strine \& Chapman, 2005; Varoneckas, et al., 2008). For example, Faubel et al. (2009) reported that extreme sleep duration: short and long ( $\leq$ 5 or $\geq 10 \mathrm{~h}$ ) is an indicator of worse HRQOL. Herrick (2010) asserted that poor quality of life and sleep-loss are highly correlated. On the other hand, good sleep quality has been linked with the quality of well-being (Jean-Louis, 2000).

\section{Conclusion}

In conclusion, poor sleep quality and fair overall health was common among WVU undergraduate college students. Study results were consistent with our hypothesized model and in line with the existent literature. The HBM and the behavioral intention (TPB) have significant roles in prediction poor sleep quality among our sample of college students. In addition, poor sleep quality was associated with increased number of unhealthy days among students. Our results extend the current knowledge regarding the prediction role of HBM and behavioral intention separately. However, uniquely we add to the current literature the influence the HBM on behavioral intention, which make a very significant combination in predicting sleep quality among college students. We have also confirmed the association between poor sleep quality and HRQOL as measured by unhealthy days during 30 days. Hence, the results of this study suggest 
that factors, such as those that make up the HBM, and behavioral intention may play an important role in achieving good sleep quality among college students in our sample and in general. Therefore, investigations or interventions concerning the health of college student should include screening for sleep quality and HRQOL. Significantly, assessment of sleep quality and/ or HRQOL can be better achieved by involving theoretical description using the HBM and behavioral intention. In order to, better understand and to improve college students' health, sleep health, productivity, wellness, quality of life, and academic achievement.

\section{Limitation}

The results of this study should be considered in light of several limitations. First, we utilized a cross-sectional research design, thus, causal relationships between study variables cannot be determined. Second, results are based on participants' self-report; therefore, subject to self-reporting accuracy, integrity and honesty. Third, we did not assess the full availability of both the HBM and TPB constructs. Finally, this study relies upon the subjective measure of sleep quality and not on objective measures such as polysomnography which provides a more accurate picture of sleep.

\section{Summary}

Poor sleep quality among college students increases the risk for lower grade point averages, compromised learning, impaired mood, and motor vehicle accidents; and associated with several unhealthy behaviors and outcomes including substances /drugs use (alcohol and medications), and weight gain. Therefore, we assessed college sleep quality in relation to the NMUPD and BMI among a large sample of college students using the fall 2010-spring 2011 national survey data (American College Health Association - National College Health Assessment (ACHA-NCHA)) (Aims1 \& 2). In aim \# 3, we used theoretical model integrating 
HBM perception constructs and behavioral intention to describe and predict college students' sleep quality among a sample of WVU undergraduate students. The goal of this dissertation was to better understand students' sleep quality. Study results can help in establishing theoretically driven interventions to promote students' sleep quality.

In summary, poor sleep was common among a large national sample of college students and among WVU college students. The NMUPD was significantly associated with poor sleep quality, and poor sleep quality significantly increases the predicted values of BMI and increases the risk of being overweight and obese. In addition, poor sleep quality was also associated with having more unhealthy days among WVU students. The HBM and the behavioral intention (TPB) have significant roles in prediction poor sleep quality among a sample of WVU college students. Our results extend the current knowledge regarding the association between NMUPD and poor sleep quality, the association between poor sleep quality and overweight and/or obesity, the association between poor sleep quality and poor HRQOL, and the significant roles of HBM and behavioral intention in explaining sleep quality among college students. Moreover, we add to the current literature the influence of the HBM on behavioral intention, which make a very significant combination in predicting sleep quality among college students. Therefore, assessment of college students' sleep quality should include screening for NMUPD, overweight and obesity, HRQOL as potential risk factors for poor sleep, and may all be interrelated. Such assessment can be better achieved by involving theoretical description using the HBM and behavioral intention, in order to, better understand and to improve college students' health, sleep quality, productivity, wellness, quality of life, and academic achievement.

In addition, dissertation findings could serve as base for further research including, applying experimental studies to objectively describe sleep behavior, in order to get more 
accurate measures of sleep quality, and to establish causal relationships between sleep and the proposed variables. In addition, this is the first known study that integrates the HBM and the behavioral intention constructs; therefore, this study will serve as a ground for additional theoretical testing using both subjective and objective measures of sleep. Finally, because the field of sleep behavior among college students particularly applying social and behavioral theories still in its infancy phase, further studies are needed to boost understanding of students' sleep and prevent the associated health issues. 


\section{REFERENCES}

Abood, D. A., Black, D. R., \& Feral, D. (2003). Nutrition Education Worksite Intervention for University Staff: Application of the Health Belief Model. Journal of Nutrition Education and Behavior, 35(5), 260-267. doi: 10.1016/s1499-4046(06)60057-2

Alexander, L., LaRosa, G., Bader, H., Garfield, S., \& Alexander, w. (2010). New Dimensions in Women's Health ( $5^{\text {th }}$ ed). Jones \& Bartlett Learning, LLC.

American College Health Association. (2011). Spring 2011Reference Group Executive Summery. In A. C. H. A. N. C. H. Assessment (Ed.).

American Sleep Association. (2015). What is Sleep? ,2015, from https://www.sleepassociation.org/patients-general-public/what-is-sleep/

Andresen, E., Catlin, T., Wyrwich, K., \& Jackson-Thompson, J. (2003). Retest reliability of surveillance questions on health related quality of life. J Epidemiol Community Health, 57, 339-343.

Andruškienė, J., Varoneckas, G., Martinkėnas, A., \& Grabauskas, V. (2008). Factors associated with poor sleep and health-related quality of life. Medicina (Kaunas), 44(3).

Anic, G. M., Titus-Ernstoff, L., Newcomb, P. A., Trentham-Dietz, A., \& Egan, K. M. (2010). Sleep duration and obesity in a population-based study. Sleep Med, 11(5), 447-451. doi: 10.1016/j.sleep.2009.11.013

Apantaku-Olajide, T.D. (2011). Abuse of prescription and over-the-counter drugs. Retrieved June 7, 2011, from www.irishpsychiatry.ie/.../forensic_essy_prize_winner_2011_web.sflb.ashx

Azevedo, C., Sousa, I., Paul, K., MacLeish, M., Mondéjar, T., Sarabia, J., . . Madrid, J. (2008). Teaching Chronobiology and Sleep Habits in School and University. Journal Compilation, 2(1).

Babcock, Q., \& Byrne, T. (2000). Student perceptions of methylphenidate abuse at a public liberal arts college. J Am Coll Health, 49(3), 143-145. doi: 10.1080/07448480009596296

Bashirian, S., Hidarnia, A., Allahverdipour, H., \& Hajizadeh, E. (2012). Application of the Theory of Planned Behavior to Predict Drug

Abuse Related Behaviors among Adolescents. Journal of Research in Health Sciences, 12(1), 54-60.

Basner, M., Müller, U., \& Elmenhorst, E.-M. (2011). Single and Combined Effects of Air, Road, and Rail Traffic Noise on Sleep and Recuperation. Sleep, 34(1), 11-23.

Benca, R. M. (2012). Sleep Disorders:The Clinician's Guide to Diagnosis and Management. New York: Oxford.

Bjorvatn, B., Sagen, I. M., Oyane, N., Waage, S., Fetveit, A., Pallesen, S., \& Ursin, R. (2007). The association between sleep duration, body mass index and metabolic measures in the Hordaland Health Study. European Sleep Research Society, 16(66-76).

Brown, F., Buboltz, W., C.,, \& Soper, B. (2002). Relationship of Sleep Hygiene Awareness, Sleep Hygiene Practices, and Sleep Quality in University Students. Behavioral Medicine, 28.

Buboltz, W., C.,, Loveland, J., Jenkins, S., M, Brown, F., Soper, B., \& Hodges, J. (2006). College student sleep: Relationship to health and academic performance. In M. Landaw, V., (Ed.), College Students: Mental Health and Coping strategies (pp. 1-39). Hauppauge, NY: Nova Science. 
Buckhalt, J., \& El-Sheikh, M. (2013). The SES Indicator. Sleep and Poverty. 2015, from http://www.apa.org/pi/ses/resources/indicator/2013/11/sleep-poverty.aspx

Burgard, S., Ailshire, J. A., \& Hughe, M. (2010). Gender and Sleep Duration among American Adults Population Studies Center: University of Michiga.

Burgard, S. A., \& Ailshire, J. A. (2012). Gender and Time for Sleep among U.S. Adults. Am Sociol Rev, 78(1), 51-69. doi: 10.1177/0003122412472048

Buysse, D. J., Reynolds, C. F., 3rd, Monk, T. H., Berman, S. R., \& Kupfer, D. J. (1989). The Pittsburgh Sleep Quality Index: a new instrument for psychiatric practice and research. Psychiatry Res, 28(2), 193-213.

Cain, N., Gradisar, M., \& Moseley, L. (2011). A motivational school-based intervention for adolescent sleep problems. Sleep Med, 12(3), 246-251. doi: 10.1016/j.sleep.2010.06.008

Cappuccio, F. P., Taggart, F. M., Kandala, N.-B., Currie, A., Peile, E., Stranges, S., \& Miller, M. A. (2008). Meta-Analysis of Short Sleep Duration and Obesity in Children and Adults. Sleep, 31(5), 619-626.

Carpenter, C. J. (2010). A meta-analysis of the effectiveness of health belief model variables in predicting behavior. Health Commun, 25(8), 661-669. doi:

10.1080/10410236.2010.521906

Carpenter, C. L., Yan, E., Chen, S., Hong, K., Arechiga, A., Kim, W. S., . . Heber, D. (2013). Body fat and body-mass index among a multiethnic sample of college-age men and women. J Obes, 2013, 790654. doi: 10.1155/2013/790654

Centers for Disease Control and Prevention. (2000). Measuring Healthy Days: Population Assessment of Health-Related Quality of Life. Atlanta, Georgia: CDC.

Centers for Disease Control and Prevention. (2008). Perceived Insufficient Rest or Sleep --- Four States, 2006 Mortality and Morbidiy Weekly Report [MMWR] (Vol. 2015).

Centers for Disease Control and Prevention. (2011a). Health-Related Quality of Life (HRQOL). HRQOL Concepts. from http://www.cdc.gov/hrqol/concept.htm

Centers for Disease Control and Prevention. (2011). Health-Related Quality of Life (HRQOL). CDC HRQOL-14 "Healthy Days Measure". from http://www.cdc.gov/hrqol/hrqol14_measure.htm

Centers for Disease Control and Prevention. (2011). Methods and Measures. Health-Related Quality of Life (HRQOL). 2015, from http://www.cdc.gov/hrqol/methods.htm

Centers for Disease Control and Prevention. (2013). How Much Sleep Do I Need? Sleep and Sleep Disorders. 2015, from http://www.cdc.gov/sleep/about_sleep/how_much_sleep.htm

Centers for Disease Control and Prevention. (2015). About adult BMI. Healthy Weight: Assessing Your Weight. 2015, from http://www.cdc.gov/healthyweight/assessing/bmi/adult_bmi/index.html

Centers for Disease Control and Prevention. (2015a). Adult Obesity Facts. Overweight and Obesity. 2015, from http://www.cdc.gov/obesity/data/adult.html

Centers for Disease Control and Prevention. (2015). Insufficient Sleep Is a Public Health Problem. Data \& Statistics. 2015, from http://www.cdc.gov/features/dssleep/

Centers for Disease Control and Prevention. (2015). Understanding the Epidemic. Prescription Drug Overdose. Retrieved January 2, 2015, 2015, from http://www.cdc.gov/drugoverdose/epidemic/index.html 
Chang, S.-P., \& Chen, Y.-H. (2014). Relationships between Sleep Quality, Physical Fitness, and Body Mass Index in College Freshmen. The Journal of Sports medicine and Physical Fitness.

Chaput, J. P. (2014). Sleep patterns, diet quality and energy balance. Physiol Behav, 134, 86-91. doi: 10.1016/j.physbeh.2013.09.006

Chaput, J. P., McNeil, J., Despres, J. P., Bouchard, C., \& Tremblay, A. (2012). Short sleep duration is associated with greater alcohol consumption in adults. Appetite, 59(3), 650655. doi: 10.1016/j.appet.2012.07.012

Chen, J., Yi, H., Liu, Z., Fan, Y., Bian, J., Guo, W., . . Sun, J. (2013). Factors associated with being overweight among Inner Mongolia medical students in China. BMJ Open, 3(12), e003900. doi: 10.1136/bmjopen-2013-003900

Chen, M. Y., Wang, E. K., \& Jeng, Y. J. (2006). Adequate sleep among adolescents is positively associated with health status and health-related behaviors. BMC Public Health, 6, 59. doi: 10.1186/1471-2458-6-59

Chong, Y., Fryar, C. D., \& Gu, Q. (2013). Prescription Sleep Aid Use Among Adults: United States, 2005-2010. In N. C. f. H. Statistics (Ed.), NCHS data brief, no 127. Hyattsville, MD.

Clegg-Kraynok, M. (2010). Nonmedical Psychostimulant Use and Sleep among Adolescents and Emerging Adults. Dessertation. (3448180). Ann Arbor, MI.

Clegg-Kraynok, M., McBean, A. L., \& Montgomery-Downs, H. E. (2011). Sleep quality and characteristics of college students who use prescription psychostimulants nonmedically. Sleep Med, 12(6), 598-602. doi: 10.1016/j.sleep.2011.01.012

Crispim, C. A., Zimberg, I. Z., dos Reis, B. G., Diniz, R. M., Tufik, S., \& de Mello, M. T. (2011). Relationship between food intake and sleep pattern in healthy individuals. J Clin Sleep Med, 7(6), 659-664. doi: 10.5664/jcsm.1476

Culnan, E., Kloss, J. D., \& Grandner, M. (2013). A prospective study of weight gain associated with chronotype among college freshmen. Chronobiol Int, 30(5), 682-690. doi: 10.3109/07420528.2013.782311

Curcio G, Ferrara M, De Gennaro L. Sleep loss, learning capacity and academic performance. Sleep Med Rev. 2006;10(5):323-337.

Dennis-Antwi, J., Adjei, S., Asare, J. B., \& Twene, R. (2003). A National Survey on Prevalenc and Social Consequencies of Substance (Drugs) use among Second Cycle and Out of School Youth in Ghana. Ghana: Ministry of Health/Ghana: World Health Organization.

Dimsdale, J. E., Norman, D., DeJardin, D., \& Wallace, M. S. (2007). The Effect of Opioids on Sleep Architecture. Journal of Clinical Sleep Medicine, 3(1), 33-36.

Dolan, C. (2013). Developmental Perspectives on Community Beliefs and Attitudes about Sleep. (Doctor of Psychology), Victoria University.

Dregan, A., \& Armstrong, D. (2010). Adolescence sleep disturbances as predictors of adulthood sleep disturbances--a cohort study. J Adolesc Health, 46(5), 482-487. doi:

10.1016/j.jadohealth.2009.11.197

Eaton, D. K., McKnight-Eily, L. R., Lowry, R., Perry, G. S., Presley-Cantrell, L., \& Croft, J. B. (2010). Prevalence of insufficient, borderline, and optimal hours of sleep among high school students - United States, 2007. J Adolesc Health, 46(4), 399-401. doi:

10.1016/j.jadohealth.2009.10.011 
Edens, K. M. (2006). The Relationship of University Students' Sleep Habits and Academic Motivation. Journal of Student Affairs Research and Practice, 43(3). doi: 10.2202/19496605.1677

Ehlers, C., Gilder, D., Criado, J., \& Caetan, R. (2010). Sleep Quality and Alcohol-Use Disorders in a Select

Population of Young-Adult Mexican Americans. Journal of Studies on Alcohol and Drugs, 71, 879-884.

Ensor, J., Oexman, R., Scott, D., Carrier, J., \& Davis, J. (2008). Evaluation of a Custom Fit Solo Construction Sleep Surface using Objective and Subjective Measures of Sleep Quality.

Evans, E. A., \& Sullivan, M. A. (2014). Abuse and misuse of antidepressants. Subst Abuse Rehabil, 5, 107-120. doi: 10.2147/SAR.S37917

Fakier, N., \& Wild, L. G. (2011). Associations among sleep problems, learning difficulties and substance use in adolescence. J Adolesc, 34(4), 717-726. doi: 10.1016/j.adolescence.2010.09.010

Faubel, R., Lopez-Garcia, E., Guallar-Castillón, P., Balboa-Castillo, T., Gutiérrez-Fisac, J., Banegas, J., \& Rodríguez-Artalejo, F. (2009). Sleep Duration and Health-Related Quality of Life among Older Adults: A Population-Based Cohort in Spain. Sleep, 32(8), 10591068.

Food Research \& Action center. (2015). Initiatives. Obesity in the U.S., 2015, from http://frac.org/initiatives/hunger-and-obesity/obesity-in-the-us/

Ford, E. S., Li, C., Wheaton, A. G., Chapman, D. P., Perry, G. S., \& Croft, J. B. (2014). Sleep duration and body mass index and waist circumference among U.S. adults. Obesity (Silver Spring), 22(2), 598-607. doi: 10.1002/oby.20558

Fredriksen, K., Rhodes, J., Reddy, R., \& Way, N. (2004). Sleepless in Chicago: Tracking the Effects of Adolescent Sleep Loss During the Middle School Years. Child Development, $75(1), 84-95$.

Friedman, E. M., Love, G. D., Rosenkranz, M. A., Urry, H. L., Davidson, R. J., Singer, B. H., \& Ryff, C. D. (2007). Socioeconomic status predicts objective and subjective sleep quality in aging women. Psychosom Med, 69(7), 682-691. doi: 10.1097/PSY.0b013e31814ceada

Fyhri, A., \& Aasvang, G. M. (2010). Noise, sleep and poor health: Modeling the relationship between road traffic noise and cardiovascular problems. Sci Total Environ, 408(21), 4935-4942. doi: 10.1016/j.scitotenv.2010.06.057

Galambos, N., Dalton, A., \& Maggs, J. (2009). Losing Sleep Over It: Daily Variation in Sleep Quantity and Quality in Canadian Students' First Semester of University. Journal of Research on Adolescence, 19(4), 741-761.

Gallasch, J., \& Gradisar, M. (2007). Relationships between sleep knowledge, sleep practice and sleep quality. Sleep and Biological Rhythms, 5(1), 63-73. doi: 10.1111/j.14798425.2006.00248.x

Gangwisch, J. E., Malaspina, D., Boden-Albala, B., \& Heymsfield, S. B. (2005). Inadequate Sleep as a Risk Factor for Obesity: Analyses of the NHANES I. Sleep, 28(10), 12891296.

Gaultney, J. F. (2010). The prevalence of sleep disorders in college students: impact on academic performance. J Am Coll Health, 59(2), 91-97. doi: 10.1080/07448481.2010.483708

Gellis, L., \& Lichstein, K. (2009). Sleep Hygiene Practices of Good and Poor Sleepers in the United States: An Internet-Based Study. Behavior Therapy, 40, 1-9. 
Glanz, K., \& Bishop, D. B. (2010). The role of behavioral science theory in development and implementation of public health interventions. Annu Rev Public Health, 31, 399-418. doi: 10.1146/annurev.publhealth.012809.103604

Glanz, K., Rimer, B., \& Viswanath, K. (2008). Health Behavior and Health Education: Theory, Research, and Practic. San Francisco, CA: Jossey-Bass: A Wiley Imprint.

Gooley, J. J., Chamberlain, K., Smith, K. A., Khalsa, S. B., Rajaratnam, S. M., Van Reen, E., . . Lockley, S. W. (2011). Exposure to room light before bedtime suppresses melatonin onset and shortens melatonin duration in humans. J Clin Endocrinol Metab, 96(3), E463472. doi: 10.1210/jc.2010-2098

Grandner, M. A., Patel, N. P., Gehrman, P. R., Xie, D., Sha, D., Weaver, T., \& Gooneratne, N. (2010). Who gets the best sleep? Ethnic and socioeconomic factors related to sleep complaints. Sleep Med, 11(5), 470-478. doi: 10.1016/j.sleep.2009.10.006

Hall, K. M., Irwin, M. M., Bowman, K. A., Frankenberger, W., \& Jewett, D. C. (2010). Illicit use of prescribed stimulant medication among college students. J Am Coll Health, 53(4), 167-174. doi: 10.3200/JACH.53.4.167-174

Halson, S. L. (2013). Nutritional Interventions to Enhance Sleep. Sports Science Exchange, 26(116), 1-5.

Hartwell, E. E., Pfeifer, J. G., McCauley, J. L., Moran-Santa Maria, M., \& Back, S. E. (2014). Sleep disturbances and pain among individuals with prescription opioid dependence. Addict Behav, 39(10), 1537-1542. doi: 10.1016/j.addbeh.2014.05.025

Harvard Medical School. (2007). Healthy Sleep. External factors that Influence Sleep. 2015

Healthy People. (2015). 2020 Topics \& Objectives. Sleep Health. Retrieved January, 2015, 2015, from http://www.healthypeople.gov/2020/topics-objectives/topic/sleep-health

Herrick, H. (2010). The Association of Insufficient Sleep with Smoking, Obesity, Physical Inactivity, and Poor Quality of Life: Results from the 2008 North Carolina Behavorial Risk Factor Surveillance System (BRFSS) Survey. Raleigh, NC: State Center for Health Statistics.

Hershner, S. D., \& Chervin, R. D. (2014). Causes and consequences of sleepiness among college students. Nat Sci Sleep, 6, 73-84. doi: 10.2147/NSS.S62907

Ho, S. C., \& Chung, J. W. (2013). The effects of caffeine abstinence on sleep: a pilot study. Appl Nurs Res, 26(2), 80-84. doi: 10.1016/j.apnr.2012.08.004

$\mathrm{Hu}, \mathrm{L} \&$ Bentler, B. (1999). Cutoff criteria for fit indexes in covariance structure analysis: Conventional criteria versus new alternatives.Structural Equation Modeling: A Multidisciplinary Journal, 6(1), 1-55, DOI: 10.1080/10705519909540118

Institute of Diabetes and Diagestive and Kidney Diseases. (2012). Overweight and Obesity Statistics. Health Statistics 2015, from http://www.niddk.nih.gov/healthinformation/health-statistics/Pages/overweight-obesity-statistics.aspx

Jacobson, B. H., Boolani, A., \& Smith, D. B. (2009). Changes in back pain, sleep quality, and perceived stress after introduction of new bedding systems. J Chiropr Med, 8(1), 1-8. doi: 10.1016/j.jcm.2008.09.002

Jean-Louis, G., Kripke, D. F., \& Ancoli-Israel, S. (2000). Sleep and Quality of Well-Being. Sleep, 23(8).

Kaloyanides, K., McCabe, S., Cranford, J., \& Teter, C. (2007). Prevalence of Illicit Use and Abuse of Prescription Stimulants, Alcohol, and Other Drugs Among College Students: Relationship with Age at Initiation of Prescription Stimulants. Pharmacotherapy, 27(5), 666-674. 
Kamath, M. G., Prakash, J., Dash, S., Chowdhury, S., Ahmed, Z. B., \& Yusof, M. Z. (2014). "Is there an Association Between Self-Reported Sleep Duration, Body Mass Index and Waist-Hip Ratio in Young Adults? A Cross-Sectional Pilot Study". J Clin Diagn Res, 8(9), BC05-07. doi: 10.7860/JCDR/2014/8918.4808

Kenney, S., Lac, A., Labrie, J., Hummer, J., \& Phan, A. (2013). Mental Health, Sleep Quality, Drinking Motives, and Alcohol-Related Consequences: A Path-Analytic Model. Journal of Studies on Alcohol and Drugs, 74, 841-851.

Kenney, S. R., LaBrie, J. W., Hummer, J. F., \& Pham, A. T. (2012). Global sleep quality as a moderator of alcohol consumption and consequences in college students. Addict Behav, 37(4), 507-512. doi: 10.1016/j.addbeh.2012.01.006

Knowlden, A. (2011). Theory of Planned Behavior Based Predictors of Sleep Intentions and Behaviors in Undergraduate College Students at a Midwestern University. University of Cincinnati.

Knowlden, A., \& Sharma, M. (2012). The Theory of Planned Behavior as a Model for Predicting the Sleep Intentions and Behaviors of Undergraduate Minority Students. International Journal of Medicine and Molecular Medicine.

Knowlden, A., \& Sharma, M. (2014). Health belief structural equation model predicting sleep behavior of employed college students. Fam Community Health, 37(4), 271-278. doi: 10.1097/FCH.0000000000000043

Kryger, M. H., Roth, T., \& Dement, W. C. (2011). Principles and Practice of Sleep Medicine. St. Louis, Missouri: Elsevier.

Lack, L. C., Gradisar, M., Van Someren, E. J., Wright, H. R., \& Lushington, K. (2008). The relationship between insomnia and body temperatures. Sleep Med Rev, 12(4), 307-317. doi: 10.1016/j.smrv.2008.02.003

Lam, r. (2005). Sleep disturbances abd depression: a challenge for antidepressants. International Clinical Psychopharmacology, 21(Suppl. 1), S25-S29.

Lauderdale, D. S., Knutson, K. L., Rathouz, P. J., Yan, L. L., Hulley, S. B., \& Liu, K. (2009). Cross-sectional and longitudinal associations between objectively measured sleep duration and body mass index: the CARDIA Sleep Study. Am J Epidemiol, 170(7), 805813. doi: 10.1093/aje/kwp230

Lee, M., Choh, A., Demerath, E., Knuston, K., Duren, D., Sherwood, R., . . Czerwinski, S. (2009). Sleep Disturbance in Relation to Health-Related Quality of Life in Adults: The Fels Longitudinal Study. The Journal of Nutrition, Health and Aging, 13(6).

Linden, M. (2011). The Theory of Planned Behavior as a Predictor of Sleep Hygiene and Sleep Quality. (3465528). Ann Arbor, MI.

Looby, A., Kassman, K. T., \& Earleywine, M. (2014). Do negative stimulant-related attitudes vary for prescription stimulants and cocaine among college students? Addict Behav, 39(6), 1100-1105. doi: 10.1016/j.addbeh.2014.03.012

Lopes, C., Esteves, A., Bittencourt, L., Tufik, S., \& Mello, M. (2008). Relationship between the quality of life and the severity of obstructive sleep apnea syndrome. Brazilian Journal of Medical and Biological Research, 41, 908-913.

Lund, H. G., Reider, B. D., Whiting, A. B., \& Prichard, J. R. (2010). Sleep patterns and predictors of disturbed sleep in a large population of college students. J Adolesc Health, 46(2), 124-132. doi: 10.1016/j.jadohealth.2009.06.016 
Magee, L., \& Hale, L. (2012). Longitudinal associations between sleep duration and subsequent weight gain: a systematic review. Sleep Med Rev, 16(3), 231-241. doi:

10.1016/j.smrv.2011.05.005

Makino, M., Hashizume, M., Yasushi, M., Tsuboi, K., \& Dennerstein, L. (2006). Factors associated with abnormal eating attitudes among female college students in Japan. Arch Womens Ment Health, 9(4), 203-208. doi: 10.1007/s00737-006-0128-X

Malinauskas, B. M., Aeby, V. G., Overton, R. F., Carpenter-Aeby, T., \& Barber-Heidal, K. (2007). A survey of energy drink consumption patterns among college students. Nutr J, 6 , 35. doi: 10.1186/1475-2891-6-35

Marshall, N. S., Glozier, N., \& Grunstein, R. R. (2008). Is sleep duration related to obesity? A critical review of the epidemiological evidence. Sleep Med Rev, 12(4), 289-298. doi: 10.1016/j.smrv.2008.03.001

Mayers, A. G., \& Baldwin, D. S. (2005). Antidepressants and their effect on sleep. Hum Psychopharmacol, 20(8), 533-559. doi: 10.1002/hup.726

McCabe, S. E., Boyd, C. J., \& Teter, C. J. (2009). Subtypes of nonmedical prescription drug misuse. Drug Alcohol Depend, 102(1-3), 63-70. doi: 10.1016/j.drugalcdep.2009.01.007

McCabe, S. E., \& Teter, C. J. (2007). Drug use related problems among nonmedical users of prescription stimulants: a web-based survey of college students from a Midwestern university. Drug Alcohol Depend, 91(1), 69-76. doi: 10.1016/j.drugalcdep.2007.05.010

McCabe, S. E., West, B. T., \& Boyd, C. J. (2013). Medical use, medical misuse, and nonmedical use of prescription opioids: results from a longitudinal study. Pain, 154(5), 708-713. doi: 10.1016/j.pain.2013.01.011

McCabe, S. E., West, B. T., Teter, C. J., \& Boyd, C. J. (2014). Trends in medical use, diversion, and nonmedical use of prescription medications among college students from 2003 to 2013: Connecting the dots. Addict Behav, 39(7), 1176-1182. doi:

10.1016/j.addbeh.2014.03.008

McKnight-Eily, L. R., Eaton, D. K., Lowry, R., Croft, J. B., Presley-Cantrell, L., \& Perry, G. S. (2011). Relationships between hours of sleep and health-risk behaviors in US adolescent students. Prev Med, 53(4-5), 271-273. doi: 10.1016/j.ypmed.2011.06.020

McLaughlin Crabtree, V., \& Williams, N. A. (2009). Normal sleep in children and adolescents. Child Adolesc Psychiatr Clin N Am, 18(4), 799-811. doi: 10.1016/j.chc.2009.04.013

Melton, B., Langdon, J., \& McDaniel, T. (2013). Sleep Trends And College Students: Does It Connect To Obesity? College Student Journal, 47(3).

Meyer, K. A., Wall, M. M., Larson, N. I., Laska, M. N., \& Neumark-Sztainer, D. (2012). Sleep duration and BMI in a sample of young adults. Obesity (Silver Spring), 20(6), 1279-1287. doi: 10.1038/oby.2011.381

Mezick, E. J., Matthews, K. A., Hall, M., Strollo, P. J., Jr., Buysse, D. J., Kamarck, T. W., . . Reis, S. E. (2008). Influence of race and socioeconomic status on sleep: Pittsburgh SleepSCORE project. Psychosom Med, 70(4), 410-416. doi: 10.1097/PSY.0b013e31816fdf21

Mezick, E. J., Wing, R. R., \& McCaffery, J. M. (2014). Associations of self-reported and actigraphy-assessed sleep characteristics with body mass index and waist circumference in adults: moderation by gender. Sleep Med, 15(1), 64-70. doi:

10.1016/j.sleep.2013.08.784 
Ming, X., Koransky, R., Kang, V., Buchman, S., Sarris, C. E., \& Wagner, G. C. (2011). Sleep insufficiency, sleep health problems and performance in high school students. Clin Med Insights Circ Respir Pulm Med, 5, 71-79. doi: 10.4137/CCRPM.S7955

Mitchell, J. A., Rodriguez, D., Schmitz, K. H., \& Audrain-McGovern, J. (2013). Sleep duration and adolescent obesity. Pediatrics, 131(5), e 1428-1434. doi: 10.1542/peds.2012-2368

Moore, D. R., Burgard, D. A., Larson, R. G., \& Ferm, M. (2014). Psychostimulant use among college students during periods of high and low stress: an interdisciplinary approach utilizing both self-report and unobtrusive chemical sample data. Addict Behav, 39(5), 987-993. doi: 10.1016/j.addbeh.2014.01.021

Moraes, W., Poyares, D., Zalcman, I., de Mello, M. T., Bittencourt, L. R., Santos-Silva, R., \& Tufik, S. (2013). Association between body mass index and sleep duration assessed by objective methods in a representative sample of the adult population. Sleep Med, 14(4), 312-318. doi: 10.1016/j.sleep.2012.11.010

Moriarty, D., Zack, M., \& Kobau, R. (2003). The Centers for Disease Control and Prevention's Healthy Days Measures - Population tracking of perceived physical and mental

health over time. Health and Quality of Life Outcomes, 1(37).

Muzet, A. (2007). Environmental noise, sleep and health. Sleep Med Rev, 11(2), 135-142. doi: 10.1016/j.smrv.2006.09.001

Nagai, M., Tomata, Y., Watanabe, T., Kakizaki, M., \& Tsuji, I. (2013). Association between sleep duration, weight gain, and obesity for long period. Sleep Med, 14(2), 206-210. doi: 10.1016/j.sleep.2012.09.024

Natal, C., Lourenco, T., Silva, L., Boscolo, R., Silva, A., Tufik, S., \& Mello, M. (2009). Gender differences in the sleep habits of 11-13 year olds. Rev Bras Psiquiatr, 31(4).

National Center for Health Statistics. (2014). Health, United States, 2013: With Special Featureon Prescription Drugs. Hyattsville, MD.

National Institute of Diabetes and Diagestive and Kidney Diseases. (2012). Understanding adult Overweight and Obesity. Weight Control \& Healthy Living. 2015, from http://www.niddk.nih.gov/health-information/health-topics/weightcontrol/understanding/Pages/understanding-adult-overweight-and-obesity.aspx

National Institute of Health. (2012a). How Much Sleep Is Enough? Sleep Deprivation and Deficiency. from https://www.nhlbi.nih.gov/health/health-topics/topics/sdd/howmuch

National Institute of Health. (2012b). What are Overweight and Obesity? Overweight and Obesity. 2015, from http://www.nhlbi.nih.gov/health/health-topics/topics/obe/

National Institute of Health. (2013). Sleep Disorders \& Insufficient Sleep: Improving Health through Research. Fact Sheet. 2015, from http://www.nhlbi.nih.gov/news/spotlight/factsheet/sleep-disorders-insufficient-sleep-improving-health-through-research

National Institute of Neurological Disorders and Stroke. (2014). Disorders A-Z. Brain Basics: Understanding Sleep. 2015, from http://www.ninds.nih.gov/disorders/brain_basics/understanding_sleep.htm

National Institute on Drug Abuse. (2014a). Waht are CNS depressants? Prescription Drug Abuse. Retrieved January 4, 2015, 2015, from http://www.drugabuse.gov/publications/research-reports/prescription-drugs/cnsdepressants/what-are-cns-depressants

National Institute on Drug Abuse. (2014b). What are Stimulants? Prescription Drug Abuse. Retrieved 01/02/2015, 2015, from http://www.drugabuse.gov/publications/researchreports/prescription-drugs/stimulants/what-are-stimulants 
National Institute on Drug Abuse. (2015). Drugs of abuse. Commonly Abused Drugs Charts. 2015, from http://www.drugabuse.gov/drugs-abuse/commonly-abused-drugs-charts

National Sleep Foundation. (2015). Bedroom. Touch. 2015, from https://sleepfoundation.org/bedroom/touch.php

National Sleep Foundation. (2015a). How Sleep Works. What Happens When You Sleep? , 2015, from http://sleepfoundation.org/how-sleep-works/what-happens-when-you-sleep

National Sleep Foundation. (2015). Sleep Hygiene. from https://sleepfoundation.org/ask-theexpert/sleep-hygiene

Newbury-Birch, D., White, M., \& Kamali, F. (2000). Factors influencing alcohol and illicit drug use amongst medical students. Drug and Alcohol Dependence, 59, 125-130.

Nursalam, M., Apriani, N. K., Has, E. M. M. a., \& Efendi, F. (2014). Sleep hygiene behavior among Balinese adolescent. Journal of Nursing Education and Practice, 4(3). doi: 10.5430/jnep.v4n3p155

Oginska, H., \& Pokorski, J. (2006). Fatigue and mood correlates of sleep length in three agesocial groups: School children, students, and employees. Chronobiol Int, 23(6), $1317-$ 1328. doi: 10.1080/07420520601089349

Ohayon, M. M. (2004). Interactions between sleep normative data and sociocultural characteristics in the elderly. J Psychosom Res, 56(5), 479-486. doi: 10.1016/j.psychores.2004.04.365

Okamoto-Mizuno, K., \& Mizuno, K. (2012). Effects of thermal environment on sleep and circadian rhythm. Journal of Physiological Anthropolo, 31(14).

Olsen, S., Smith, S., Oei, T., \& Douglas, J. (2008). Health belief model predicts adherence to CPAP before experience with CPAP. Eur Respir J, 32(3), 710-717. doi: 10.1183/09031936.00127507

Olsen, S., Smith, S., \& Oei, T. P. (2008). Adherence to continuous positive airway pressure therapy in obstructive sleep apnoea sufferers: a theoretical approach to treatment adherence and intervention. Clin Psychol Rev, 28(8), 1355-1371. doi: 10.1016/j.cpr.2008.07.004

Painter, J. E., Borba, C. P., Hynes, M., Mays, D., \& Glanz, K. (2008). The use of theory in health behavior research from 2000 to 2005: a systematic review. Ann Behav Med, 35(3), 358362. doi: 10.1007/s12160-008-9042-y

Pasch, K., Laska, M., Lytle, L., \& Moe, S. (2010). Adolescent Sleep, Risk Behaviors, and Depressive Symptoms:

Are They Linked? Am J Health Behav., 34(2), 237-248.

Patel, S. R., \& Hu, F. B. (2008). Short sleep duration and weight gain: a systematic review. Obesity (Silver Spring), 16(3), 643-653. doi: 10.1038/oby.2007.118

Peuhkuri, K., Sihvola, N., \& Korpela, R. (2012). Diet promotes sleep duration and quality. Nutr Res, 32(5), 309-319. doi: 10.1016/j.nutres.2012.03.009

Phillips, B., \& Mannino, D. (2005). Correlates of Sleep Complaints in Adults: The ARIC Study. Journal of Clinical Sleep Medicine, 1(3), 277-283.

Popovici, I., \& French, M. T. (2013). Binge drinking and sleep problems among young adults. Drug \& Alcohol Dependence, 132(1), 207-215. doi: 10.1016/j.drugalcdep.2013.02.001

Pratt, L. A., Brody, D. J., \& Gu, Q. (2011). Antidepressant Use in Persons Aged 12 and Over: United States, 2005-2008 NCHS Data Brief, No.76. Hyattsville, MD: National Center for Health Statistics. 
Preišegolavičiūtè, E., Leskauskas, D., \& Adomaitienè, V. (2010). Associations of quality of sleep with lifestyle factors and profile of studies among Lithuanian students. Medicina (Kaunas), 46(7), 482-489.

Quick, V., Byrd-Bredbenner, C., Brown, O., Colby, S., Horacek, T., Shoff, S., . . Kidd, T. (2013). Relationships among Sleep Duration, Weight-Related Behaviors, and BMI in College Students. Journal of the Academy of Nutrition and Dietetics, 113(9), A78. doi: 10.1016/j.jand.2013.06.270

Quick, V., Shoff, S., Lohse, B., White, A., Horacek, T., \& Greene, G. (2015). Relationships of eating competence, sleep behaviors and quality, and overweight status among college students. Eat Behav, 19, 15-19. doi: 10.1016/j.eatbeh.2015.06.012

Ram, S., Seirawan, H., Kumar, S. K., \& Clark, G. T. (2010). Prevalence and impact of sleep disorders and sleep habits in the United States. Sleep Breath, 14(1), 63-70. doi: 10.1007/s11325-009-0281-3

Riedel, B. W. (2000). Sleep hygiene. In K. L. L. C. M. Morin (Ed.), Treatment of late-life insomnia (pp. 125-146). Thousand Oaks, CA, US: Sage Publications, Inc.

Robotham, D., Chakkalackal, L., \& Cyhlarova, E. (2011). Sleep Matters: The impact of sleep on health and wellbeing.

Ruiter, M. E., Decoster, J., Jacobs, L., \& Lichstein, K. L. (2011). Normal sleep in AfricanAmericans and Caucasian-Americans: A meta-analysis. Sleep Med, 12(3), 209-214. doi: 10.1016/j.sleep.2010.12.010

Sanchez, S. E., Martinez, C., Oriol, R. A., Yanez, D., Castaneda, B., Sanchez, E., Williams, M. A. (2013). Sleep Quality, Sleep Patterns and Consumption of Energy Drinks and Other Caffeinated Beverages among Peruvian College Students. Health (Irvine Calif), 5(8B), 26-35. doi: 10.4236/health.2013.58A2005

Sax, L., Gilmartin, S., \& Bryant, A. (2003). Asswssing Response Rates and Nonresponse Bias in Web and paper Surveys. in Higher Education, 44(4), 209-214. doi: 10.1016/j.sleep.2010.12.010

Schlangen, L. (2014). The effect of light on our sleep/wake cycle: Philips.

Schlarb, A. A., Kulessa, D., \& Gulewitsch, M. D. (2012). Sleep characteristics, sleep problems, and associations of self-efficacy among German university students. Nat Sci Sleep, 4, 1-7. doi: 10.2147/NSS.S27971

Shoff, S., Nuss, E., Horacek, T., Boyle, C., Lohse, B., Patterson, J., . . Greene, G. (2009). Sleep Quality is Associated with Eating Behavior in 18-24 year old College Students. Journal of Nutrition Education and Behavior, 41(4), S8-S9. doi: 10.1016/j.jneb.2009.03.117

Singleton, R. A., \& Wolfson, A. (2009). Alcohol Consumption, Sleep, and Academic PerformanceAmong College Students. Journal of Studies on Alcohol and Drugs, 70, 355363.

Spaeth, A. M., Dinges, D. F., \& Goel, N. (2013). Effects of Experimental Sleep Restriction on Weight Gain, Caloric Intake, and Meal Timing in Healthy Adults. Sleep, 36(7), 981-990. doi: $10.5665 /$ sleep. 2792

Stanko, K. (2013). Predicting Sleep Duration in College Students: A Reasoned Action approach. (Master), Ball State University MUNCIE, INDIANA.

Storfer-Isser, A., Patel, S. R., Babineau, D. C., \& Redline, S. (2012). Relation between sleep duration and BMI varies by age and sex in youth age 8-19. Pediatr Obes, 7(1), 53-64. doi: 10.1111/j.2047-6310.2011.00008.x 
Strine, T. W., \& Chapman, D. P. (2005). Associations of frequent sleep insufficiency with health-related quality of life and health behaviors. Sleep Med, 6(1), 23-27. doi: 10.1016/j.sleep.2004.06.003

Substanc Abuse and Mental Health Services Administration. (2015). Prescription Drug Misuse and abuse. Topics. Retrieved January, 2015, 2015, from http://www.samhsa.gov/prescription-drug-misuse-abuse

Suen, L., Tam, W., \& Hon, K. (2010). Association of sleep hygiene-related factors and sleep quality among university students in Hong Kong. Hong Kong Med J, 16(3).

Sutton, S. (2002). Health Behavior: Psychosocial Theories.

Sweileh, W. M., Ali, I., Sawalha, A. F., Abu-Tah, A. S., Zyoud, S. e. H., \& Al-Jabi, S. W. (2012). Gender differences in sleep habits and sleep-related problems in Arab Palestinian university students. International Journal on Disability and Human Development, 11(3). doi: 10.1515/ijdhd-2012-0041

Taheri, S., Lin, L., Austin, D., Young, T., \& Mignot, E. (2004). Short sleep duration is associated with reduced leptin, elevated ghrelin, and increased body mass index. PLoS Med, 1(3), e62. doi: 10.1371/journal.pmed.0010062

Taylor, D., Bury, M., Campling, N., Carter, S., Garfied, S., Newbould, J., \& Rennie, T. (2006). A Review of the use of the Health Belief Model (HBM), the Theory of Reasoned Action (TRA), the Theory of Planned Behavior (TPB) and the Trans-Theoretical Model (TTM) to study and predict health related behavior change.

Taylor, D. J., \& Bramoweth, A. D. (2010). Patterns and consequences of inadequate sleep in college students: substance use and motor vehicle accidents. J Adolesc Health, 46(6), 610-612. doi: 10.1016/j.jadohealth.2009.12.010

Teter, C., McCabe, S., LaGrange, K., Cranford, J., \& Boyd, C. (2006). Illicit Use of Specific Prescription Stimulants Among College Students: Prevalence, Motives, and Routes of Administration. Pharmacotherapy, 26(10).

Teter, C. J., McCabe, S. E., Cranford, J. A., Boyd, C. J., \& Guthrie, S. K. (2010). Prevalence and motives for illicit use of prescription stimulants in an undergraduate student sample. $J \mathrm{Am}$ Coll Health, 53(6), 253-262. doi: 10.3200/JACH.53.6.253-262

Todigala, K., Holcomb, D., \& Kanneganti, M. (2008). Relationship between sleep and body mass index (BMI) among college students. Paper presented at the 136 th ${ }_{A}$ PHA Annual Meeting and Exposition, San Diego.

Tran, J., Lertmaharit, S., Lohsoonthorn, V., Pensuksan, W. C., Rattananupong, T., Tadesse, M. G., .. . Williams, M. A. (2014). Daytime Sleepiness, Circadian Preference, Caffeine Consumption and Use of Other Stimulants among Thai College Students. J Public Health Epidemiol, 8(6), 202-210. doi: 10.5897/JPHE2014.0620

Tsai, L.-L., \& Li, S.-P. (2004). Sleep patterns in college students. Journal of Psychosomatic Research, 56(2), 231-237. doi: 10.1016/s0022-3999(03)00507-5

United State National Library of Medicine. (2015). ObesityHealth Topics. 2015, from https://www.nlm.nih.gov/medlineplus/obesity.html\#

United States Drug Enforcement Administration. (2015). Drugs of Abuse-A DEA Resource Guide. Retrieved December 8, 2016, from https://www.dea.gov/pr/multimedialibrary/publications/drug_of_abuse.pdf\#page $=46$

Vargas, P. A., Flores, M., \& Robles, E. (2014). Sleep quality and body mass index in college students: the role of sleep disturbances. J Am Coll Health, 62(8), 534-541. doi: $10.1080 / 07448481.2014 .933344$ 
Vela-Bueno A, Fernandez-Mendoza J, Olavarrieta-Bernardino S. Sleep patterns in the transition from adolescence to young adulthood. Sleep Med Clin. 2009;4(1):77-85.

Waye, K., Clow, A., Edwards, S., Hucklebridge, F., \& Rylander, R. (2003). Effects of nighttime low frequency noise on the cortisol response to awakening and subjective sleep quality. Life Science, 72, 863-875.

Wechsler, H., \& Wuethrich, B. (2002). Dying to Drink: Confronting Binge Drinking on College Campuses. Emmaus, PA: Rodale.

Wells, M., \& Vaughn, B. (2012). Poor Sleep Challenging the Health of a Nation. Neurodiagn J, 52, 233-249.

West Virginia University. (2015). About WVU. WVU Facts. 2015, from http://about.wvu.edu/wvu-facts

Wheaton, A. G., Perry, G. S., Chapman, D. P., McKnight-Eily, L. R., Presley-Cantrell, L. R., \& Croft, J. B. (2011). Relationship between body mass index and perceived insufficient sleep among U.S. adults: an analysis of 2008 BRFSS data. BMC Public Health, 11, 295. doi: 10.1186/1471-2458-11-295

Wilens, T. E., Adler, L. A., Adams, J., Sgambati, S., Rotrosen, J., Sawtelle, R., . . Fusillo, S. (2008). Misuse and diversion of stimulants prescribed for ADHD: a systematic review of the literature. J Am Acad Child Adolesc Psychiatry, 47(1), 21-31. doi: 10.1097/chi.0b013e31815a56f1

Wilson, S., \& Argyropoulos, S. (2005). Antidepressants and Sleep: A Qualitative Review of the Literature. Drugs, 65(7).

Wong, M. M., Brower, K. J., Nigg, J. T., \& Zucker, R. A. (2010). Childhood sleep problems, response inhibition, and alcohol and drug outcomes in adolescence and young adulthood. Alcohol Clin Exp Res, 34(6), 1033-1044. doi: 10.1111/j.1530-0277.2010.01178.x

World Health Organization. (2015). Substance abuse. Health Topics. 2015, from http://www.who.int/topics/substance_abuse/en/

Wuertz, C., Chia, Y., \& Lee, J. (2012). Relationship of Body Mass Index and Activity Level with Sleep Quality Among College Women. DISCOVERY: Georgia State Honors College UndergraduateResearch Journal, 1(1).

Xiang, Y. T., Ma, X., Lu, J. Y., Cai, Z. J., Li, S. R., Xiang, Y. Q., . . Ungvari, G. S. (2009). Relationships of sleep duration with sleep disturbances, basic socio-demographic factors, and BMI in Chinese people. Sleep Med, 10(10), 1085-1089. doi: 10.1016/j.sleep.2009.03.002

Yamaguchi, M., Uemura, H., Katsuura-Kamano, S., Nakamoto, M., Hiyoshi, M., Takami, H., .. . Arisawa, K. (2013). Relationship of dietary factors and habits with sleep-wake regularity. Asia Pac J Clin Nutr, 22(3), 457-465. doi: 10.6133/apjen.2013.22.3.01

Yi, H., Shin, K., \& Shin, C. (2006). Development of the Sleep Quality Scale. J. Sleep Res, 15, 309-316. 
Appendix A

Aim 1, Results' Tables 
Table 1. Sample Characteristics ( $F=$ Frequency, $\%=$ percentage)

\begin{tabular}{|c|c|c|c|c|}
\hline \multicolumn{2}{|c|}{ Characteristic } & $\begin{array}{c}\text { Male } \\
{[n=46,062(34.7 \%)]}\end{array}$ & $\begin{array}{c}\text { Female } \\
{[n=86,593(65.3 \%)]}\end{array}$ & $\begin{array}{l}\text { Overall } \\
{[N=133,211(100 \%)]} \\
\text { Frequency Missing = 2, } 663\end{array}$ \\
\hline \multicolumn{5}{|c|}{ Demographic } \\
\hline \multicolumn{5}{|c|}{$\mathrm{N}=135,874 ;$ Age $\mathrm{M}(\mathrm{SD})=22(6)$} \\
\hline \multirow[t]{7}{*}{ Race/Ethnicity F (\%) } & White & $31037(34.6)$ & $58566(65.4)$ & $89,603(68.1)$ \\
\hline & Black & $2125(29.6)$ & $5045(70.4)$ & $7,170(5.5)$ \\
\hline & Hispanic or Latino & $3208(33.7)$ & $6324(66.3)$ & $9,532(7.2)$ \\
\hline & $\begin{array}{l}\text { Asian or Pacific } \\
\text { Islander }\end{array}$ & $5794(38.2)$ & $9374(61.8)$ & $15,168(11.5)$ \\
\hline & $\begin{array}{l}\text { American Indian, } \\
\text { Alaskan Native, or } \\
\text { Native Hawaiian }\end{array}$ & $727(34.8)$ & $1361(65.2)$ & $2,088(1.6)$ \\
\hline & $\begin{array}{l}\text { Biracial or } \\
\text { Multiracial }\end{array}$ & $1370(29.4)$ & $3285(70.6)$ & $4,655(3.6)$ \\
\hline & Other & $1337(40.1)$ & $2001(59.9)$ & $3,338(2.5)$ \\
\hline \multicolumn{2}{|c|}{ BMI $M(\mathrm{SD})$} & $24.8(4.8)$ & $23.9(5.2)$ & $24.2(5.1)$ \\
\hline \multicolumn{5}{|c|}{ Substance use (past 30 days) F (\%) } \\
\hline \multicolumn{2}{|l|}{ Cigarette smoking } & $8,543(43.0)$ & $11,344(57.0)$ & $19,887(15.0)$ \\
\hline \multicolumn{2}{|l|}{ Alcohol use } & $29,542(34.8)$ & $55,353(65.2)$ & $84,895(65.6)$ \\
\hline \multicolumn{2}{|l|}{ Marijuana use } & $8,599(42.4)$ & $11,698(57.6)$ & $20,297(15.4)$ \\
\hline \multicolumn{5}{|c|}{ Diagnosed Medical Disorders in the Past 12 Months and the perceived overall stress level $F(\%)$} \\
\hline \multicolumn{2}{|c|}{ Attention Deficit Hyperactivity Disorder (ADHD) } & $2,258(38.7)$ & $3,579(61.3)$ & $5,837(4.4)$ \\
\hline \multicolumn{2}{|l|}{ Insomnia } & $1,358(27.9)$ & $3,511(72.1)$ & $4,869(3.7)$ \\
\hline \multicolumn{2}{|l|}{ Sleep-related Disorders } & $921(35.0)$ & $1,709(65.0)$ & $2,630(2.0)$ \\
\hline \multicolumn{2}{|l|}{ Depression } & $6,014(26.0)$ & $17,115(74.0)$ & $23,129(18.0)$ \\
\hline \multicolumn{2}{|l|}{ Stress } & $44,162(34.0)$ & $85,487(66.0)$ & $129,649(98.3)$ \\
\hline
\end{tabular}


Table.2 Overall and By Gender Distribution of Sample Sleep Behaviors (Dependent variables) and Non-Medical Use of Prescription Drugs [NMUPD] (Independent Variables),

$(F=$ Frequency, $\%=$ percentage $)$

\begin{tabular}{|c|c|c|c|c|}
\hline \multicolumn{2}{|r|}{ Variable } & Male (\%) & Female (\%) & Overall (\%) \\
\hline \multicolumn{5}{|c|}{ Sleep Variables (in the past 7 days), Number of Days } \\
\hline \multirow{3}{*}{ Enough Sleep } & 0-1 Day "Poor" & $8865(30.4)$ & $20280(69.6)$ & $29145(22.1)$ \\
\hline & 2-5 Days "Middle" & $30633(34.8)$ & $57254(65.2)$ & $87887(66.6)$ \\
\hline & 6-7 Days "Optimal" & $6239(42.0)$ & $8626(58.0)$ & $14865(11.3)$ \\
\hline \multirow[t]{3}{*}{ Early Awakening } & 0-1 Day “Optimal” & $34842(36.3)$ & $61197(63.7)$ & $96039(72.9)$ \\
\hline & 2-5 Days "Middle" & $9698(30.3)$ & $22304(69.7)$ & $32002(24.3)$ \\
\hline & 6-7 Days "Poor" & $1113(30.6)$ & $2523(69.4)$ & $3636(2.8)$ \\
\hline \multirow[t]{3}{*}{ Daytime Sleepiness } & 0-1 Day “Optimal” & $12398(43.8)$ & $15926(56.2)$ & $28324(21.5)$ \\
\hline & 2-5 Days "Middle" & $27496(33.5)$ & $54691(66.5)$ & $82187(62.3)$ \\
\hline & 6-7 Days "Poor" & $5834(27.3)$ & $15527(72.7)$ & $21361(16.2)$ \\
\hline \multirow{3}{*}{$\begin{array}{l}\text { Difficulty Falling } \\
\text { Asleep }\end{array}$} & 0-1 Day "Optimal" & $30547(36.1)$ & $53981(63.9)$ & $84528(64.1)$ \\
\hline & 2-5 Days "Middle" & $12140(32.3)$ & $25447(67.7)$ & $37587(28.5)$ \\
\hline & 6-7 Days "Poor" & $3021(31.0)$ & $6697(69.0)$ & $9718(7.4)$ \\
\hline \multirow[t]{2}{*}{$\begin{array}{l}\text { Problem with } \\
\text { Daytime sleepiness }\end{array}$} & $\begin{array}{l}\text { No Problem } \\
\text { "Optimal" }\end{array}$ & $6198(46.4)$ & $7168(53.6)$ & $13366(10.1)$ \\
\hline & $\begin{array}{l}\text { Little to Very big } \\
\text { problem "Poor" }\end{array}$ & $39573(33.4)$ & $78990(66.6)$ & $118563(89.9)$ \\
\hline \multicolumn{5}{|c|}{ Prescription drugs (past 12 months) } \\
\hline \multicolumn{2}{|c|}{ Antidepressant use } & $1,264(31.5)$ & $2,742(68.5)$ & $4,006(3.0)$ \\
\hline \multicolumn{2}{|l|}{ Painkiller use } & $3,915(39.8)$ & $5,911(60.2)$ & $9,826(7.5)$ \\
\hline \multicolumn{2}{|l|}{ Sedative use } & $3,781(39.1)$ & $5,881(60.9)$ & $9,661(7.4)$ \\
\hline \multicolumn{2}{|l|}{ Stimulant use } & $1,998(37.4)$ & $3,340(62.6)$ & $5,338(4.1)$ \\
\hline \multicolumn{2}{|l|}{ Any NMUPD } & $6,786(36.8)$ & $11,651(63.2)$ & $18,437(14.1)$ \\
\hline
\end{tabular}


Table 3. Overall and by gender Ordinal Regression Analysis (OR $(95 \%$ CI)) for the non-medical use of prescription drugs (NMUPD) as variables predicting sleep behaviors among college students $(N=135,874$; adjusting for covariates ${ }^{\Delta}$ )

\begin{tabular}{|c|c|c|c|c|c|}
\hline \multirow{2}{*}{$\begin{array}{l}\text { NMUPD } \\
\text { Variables } \\
\text { Yes vs. No }\end{array}$} & \multicolumn{5}{|c|}{ Responses: Sleep Variables } \\
\hline & $\begin{array}{l}\text { Enough Sleep } \\
\left(\mathrm{OR}^{\wedge}\right)\end{array}$ & $\begin{array}{l}\text { Problem with } \\
\text { Daytime Sleepiness } \\
\left(\mathrm{OR}^{\wedge \wedge}\right)\end{array}$ & $\begin{array}{l}\text { Early Awakening } \\
\left(\mathrm{OR}^{\wedge \wedge \wedge}\right)\end{array}$ & $\begin{array}{l}\text { Daytime Sleepiness } \\
\left(\mathrm{OR}^{\wedge \wedge \wedge}\right)\end{array}$ & $\begin{array}{l}\text { Difficulty Falling } \\
\text { Asleep } \underline{\left(\mathrm{OR}^{\wedge \wedge \wedge}\right)}\end{array}$ \\
\hline \multicolumn{6}{|c|}{ Antidepressants } \\
\hline Overall & $0.98(0.92-1.05)$ & $0.99(0.93-1.07)$ & $1.02(0.95-1.09)$ & $1.07(0.99-1.14)$ & $1.04(0.97-1.11)$ \\
\hline Male & $0.96(0.85-1.08)$ & $1.04(0.92-1.18)$ & $1.07(0.95-1.22)$ & $1.01(0.89-1.13)$ & $1.01(0.89-1.14)$ \\
\hline Female & $0.99(0.92-1.08)$ & $0.97(0.89-1.05)$ & $0.99(0.91-1.07)$ & $1.08(0.99-1.17)$ & $1.05(0.97-1.14)$ \\
\hline \multicolumn{6}{|c|}{ Painkillers } \\
\hline Overall & $0.84 * * *(0.80-0.88)$ & $0.98(0.93-1.02)$ & $1.28 * * *(1.22-1.34)$ & $1.16 * * *(1.11-1.22)$ & $1.27 * * *(1.21-1.33)$ \\
\hline Male & $0.87 * *(0.80-0.93)$ & $0.98(0.91-1.06)$ & $1.34 * * *(1.24-1.45)$ & $1.16 * * *(1.08-1.25)$ & $1.33 * * *(1.23-1.43)$ \\
\hline Female & $0.83 * * *(0.78-0.87)$ & $0.98(0.92-1.04)$ & $1.24 * * *(1.17-1.31)$ & $1.17 * * *(1.11-1.24)$ & $1.23 * * *(1.16-1.30)$ \\
\hline \multicolumn{6}{|c|}{ Sedatives } \\
\hline Overall & $1.07(1.01-1.14)$ & $1.10 *(1.03-1.17)$ & $1.12 * *(1.05-1.19)$ & $0.98(0.92-1.04)$ & $1.17 * * *(1.09-1.24)$ \\
\hline Male & $1.08(0.97-1.19)$ & $1.09(0.98-1.22)$ & $1.07(0.96-1.19)$ & $0.96(0.86-1.06)$ & $1.13(1.02-1.26)$ \\
\hline Female & $1.07(0.99-1.16)$ & $1.09(1.00-1.18)$ & $1.14 *(1.05-1.24)$ & $0.99(0.92-1.07)$ & $1.18 * * *(1.09-1.27)$ \\
\hline \multicolumn{6}{|c|}{ Stimulants } \\
\hline Overall & $0.93 *(0.89-0.97)$ & $0.97(0.93-1.02)$ & $1.10 * * *(1.05-1.16)$ & $1.13 * * *(1.08-1.18)$ & $1.10 * * *(1.05-1.15)$ \\
\hline Male & $0.88 * *(0.82-0.95)$ & $0.93(0.86-1.01)$ & $1.15 * *(1.06-1.25)$ & $1.20 * * *(1.11-1.29)$ & $1.21 * * *(1.12-1.30)$ \\
\hline Female & $0.96(0.91-1.01)$ & $0.99(0.94-1.06)$ & $1.07(1.01-1.14)$ & $1.09 * *(1.04-1.16)$ & $1.04(0.99-1.11)$ \\
\hline \multicolumn{6}{|c|}{ Use at least one } \\
\hline Overall & $0.86 * * *(0.83-0.88)$ & $0.98(0.95-1.02)$ & $1.28 * * *(1.24-1.33)$ & $1.23 * * *(1.19-1.26)$ & $1.32 * * *(1.28-1.37)$ \\
\hline Male & $0.84 * * *(0.80-0.89)$ & $0.97(0.92-1.03)$ & $1.33 * * *(1.26-1.41)$ & $1.22 * * *(1.16-1.29)$ & $1.44 * * *(1.36-1.52)$ \\
\hline Female & $0.87 * * *(0.83-0.90)$ & $0.99(0.95-1.03)$ & $1.26 * * *(1.21-1.31)$ & $1.23 * * *(1.18-1.28)$ & $1.26 * * *(1.22-1.31)$ \\
\hline
\end{tabular}

$* p<.01 ; * * p<.001 ; * * * p<.0001$

OR interpretation:

$\wedge$ Less days of having Enough Sleep "Poor" $<1>$ More days of having Enough Sleep "Optimal".

$\wedge \wedge$ No problem with Daytime Sleepiness "Optimal" $<\mathbf{1}>$ little to a very big problem with Daytime

Sleepiness "Poor".

$\wedge \wedge \wedge$ Less days of Early Awakening, Daytime Sleepiness, and Difficulty Falling Asleep "Optimal" $<1>$ more days of Early Awakening, Daytime Sleepiness, and Difficulty Falling Asleep "Poor”.

${ }^{\Delta}$ Controlling for race; gender; age; BMI; cigarette, alcohol and marijuana use; being diagnosed for ADHD (Attention Deficit Hyperactivity Disorder), insomnia, sleep disorders, and depression; and the overall stress level. We excluded gender when modelling by gender. And, when we modelled one of the NMUPD as a predictor we controlled for the other NMUPD. 
Appendix B

Aim2, Results' Tables and Figures 


\section{Descriptive results: sample characteristics}

A- Demographic and Covariates Variables [ $\mathrm{N}=135,874$; Age $\mathrm{M}(\mathrm{SD})$ 22(6)]:

Table 2a: Demographic: Gender, Race, and Age.

$(\mathrm{F}=$ Frequency, $\%=$ percentage $)$

\begin{tabular}{|c|c|c|c|c|}
\hline \multicolumn{2}{|c|}{ Characteristics } & $\begin{array}{l}\text { Male } \\
{[n=46,062(34.7 \%)]}\end{array}$ & $\begin{array}{l}\text { Female } \\
{[n=86,593(65.3 \%)]}\end{array}$ & $\begin{array}{l}\text { Overall } \\
{[N=133,211(100 \%)]} \\
\text { Frequency Missing = } \\
2,663\end{array}$ \\
\hline \multirow{2}{*}{\multicolumn{5}{|c|}{$N=135,874 ; \operatorname{Age} M(S D)=22(6)$}} \\
\hline & & & & I.Demographic \\
\hline \multirow{7}{*}{$\begin{array}{l}\text { Race/Ethnicity } F \\
(\%)\end{array}$} & White & $31037(34.6)$ & $58566(65.4)$ & $89,603(68.1)$ \\
\hline & Black & $2125(29.6)$ & $5045(70.4)$ & $7,170(5.5)$ \\
\hline & Hispanic or Latino & $3208(33.7)$ & $6324(66.3)$ & $9,532(7.2)$ \\
\hline & Asian or Pacific Islander & $5794(38.2)$ & $9374(61.8)$ & $15,168(11.5)$ \\
\hline & $\begin{array}{l}\text { American Indian, Alaskan } \\
\text { Native, or Native Hawaiian }\end{array}$ & $727(34.8)$ & $1361(65.2)$ & 2, $088(1.6)$ \\
\hline & Biracial or Multiracial & $1370(29.4)$ & $3285(70.6)$ & $4,655(3.6)$ \\
\hline & Other & $1337(40.1)$ & $2001(59.9)$ & $3,338(2.5)$ \\
\hline
\end{tabular}


Table 1b: Covariates: Substance use (Cigarette, Alcohol, and Marijuana) the Nonmedical use of prescription drugs (Antidepressants, Painkiller, Stimulants, and Sedatives), Diagnosed Medical Disorders, and the Overall Perceived Stress Level.

\begin{tabular}{|c|c|c|c|c|}
\hline \multicolumn{2}{|l|}{ Characteristics } & Male & Female & Overall \\
\hline \multicolumn{5}{|l|}{ Substance use (past 30 days) $F(\%)$} \\
\hline \multirow[t]{2}{*}{ Cigarette smoking } & Use & $8,543(43.0)$ & $11,344(57.0)$ & $19,887(15.0)$ \\
\hline & No & $37,254(33.2)$ & $74,915(66.8)$ & $112,169(85.0)$ \\
\hline \multirow[t]{2}{*}{ Alcohol use } & Use & $29,542(34.8)$ & $55,353(65.2)$ & $84,895(65.6)$ \\
\hline & No & $16,101(34.6)$ & $30,504(65.4)$ & $46,605(35.4)$ \\
\hline \multirow[t]{2}{*}{ Marijuana use } & Use & $8,599(42.4)$ & $11,698(57.6)$ & $20,297(15.4)$ \\
\hline & No & $37,117(33.3)$ & $74,367(66.7)$ & $111,484(84.6)$ \\
\hline \multicolumn{5}{|c|}{ The Nonmedical Use of Prescription Drugs (past 12 months) $F(\%)$} \\
\hline \multirow[t]{2}{*}{ Antidepressant use } & Use & $1,264(31.5)$ & $2,742(68.5)$ & $4,006(3.0)$ \\
\hline & No & $44,492(34.8)$ & $83,397(65.2)$ & $127,889(97.0)$ \\
\hline \multirow[t]{2}{*}{ Painkillers use } & Use & $3,915(39.8)$ & $5,911(60.2)$ & $9,826(7.5)$ \\
\hline & No & $41,763(34.3)$ & $80,162(65.7)$ & $121,925(92.5)$ \\
\hline \multirow[t]{2}{*}{ Stimulants use } & Use & $3,781(39.1)$ & $5,881(60.9)$ & $9,661(7.4)$ \\
\hline & No & $41,810(34.3)$ & $80,020(65.7)$ & $121,830(92.6)$ \\
\hline \multirow[t]{2}{*}{ Sedatives use } & Use & $1,998(37.4)$ & $3,340(62.6)$ & $5,338(4.1)$ \\
\hline & No & $43,620(34.6)$ & $82,634(65.4)$ & $126,254(95.9)$ \\
\hline \multirow[t]{2}{*}{ Use at least one } & Use & $6,786(36.8)$ & $11,651(63.2)$ & $18,437(14.1)$ \\
\hline & No & $38,609(34.3)$ & $73,848(65.7)$ & $112,457(85.9)$ \\
\hline \multicolumn{5}{|c|}{ Diagnosed Medical Disorders in the Past 12 Months and the perceived overall stress level F (\%) } \\
\hline \multirow[t]{2}{*}{ Attention Deficit Hyperactivity Disordr (ADHD) } & Yes & $2,258(38.7)$ & $3,579(61.3)$ & $5,837(4.4)$ \\
\hline & No & $43,366(34.5)$ & $82,396(65.5)$ & $125,762(95.6)$ \\
\hline \multirow[t]{2}{*}{ Insomnia } & Yes & $1,358(27.9)$ & $3,511(72.1)$ & $4,869(3.7)$ \\
\hline & No & $44,234(34.9)$ & $82,404(65.1)$ & $126,638(96.3)$ \\
\hline \multirow[t]{2}{*}{ Sleep-related Disorders } & Yes & $921(35.0)$ & $1,709(65.0)$ & $2,630(2.0)$ \\
\hline & No & $44,525(34.6)$ & $84,001(65.4)$ & $128,536(98.0)$ \\
\hline \multirow[t]{2}{*}{ Depression } & Yes & $6,014(26.0)$ & $17,115(74.0)$ & $23,129(18.0)$ \\
\hline & No & $37,947(36.0)$ & $67,211(64.0)$ & $105,158(82.0)$ \\
\hline \multirow[t]{2}{*}{ Stress } & Yes & $44,162(34.0)$ & $85,487(66.0)$ & $129,649(98.3)$ \\
\hline & No & $1,562(70.1)$ & $666(29.9)$ & $2,228(1.7)$ \\
\hline
\end{tabular}


Table 2c: Covariates: Exercise (in the past 7 days); and exercise, diet and pills used to lose weight (within the last 30 days).

\begin{tabular}{|c|c|c|c|c|c|}
\hline \multicolumn{3}{|c|}{ Characteristics } & Male & Female & Overall \\
\hline \multicolumn{6}{|c|}{ Exercise (in the past 7 days), Number of Days } \\
\hline \multirow{4}{*}{$\begin{array}{l}\text { Moderate intensity cardio or } \\
\text { aerobic exercises for at least } 30 \\
\text { minutes }\end{array}$} & \multicolumn{2}{|c|}{ M(SD) } & \multicolumn{3}{|c|}{$2.5(2.1)$} \\
\hline & \multirow{3}{*}{$\mathrm{F}(\%)$} & 0-1 Day & $16,566(33.9)$ & $32,362(66.1)$ & $48,928(37.2)$ \\
\hline & & 2-5 Days & $23,788(34.3)$ & $45,614(65.7)$ & $69,402(52.8)$ \\
\hline & & 6-7 Days & $5,100(39.4)$ & $7,851(60.6)$ & $12,951(10.0)$ \\
\hline \multirow{4}{*}{$\begin{array}{l}\text { Vigorous intensity cardio or } \\
\text { aerobic exercises for at least } 20 \\
\text { minutes }\end{array}$} & \multicolumn{2}{|c|}{ M(SD) } & \multicolumn{3}{|c|}{$1.7(1.9)$} \\
\hline & \multirow{3}{*}{$\mathrm{F}(\%)$} & 0-1 Day & $22,643(31.4)$ & $49,487(68.6)$ & $72,130(55.0)$ \\
\hline & & 2-5 Days & $19,940(38.5)$ & $31,911(61.5)$ & $51,851(39.6)$ \\
\hline & & 6-7 Days & $2,889(40.8)$ & $4,200(59.2)$ & $7,089(5.4)$ \\
\hline \multirow{4}{*}{$\begin{array}{l}\text { 8-10 strength training exercises for } \\
\text { 8-12 repetitions each }\end{array}$} & \multicolumn{2}{|c|}{ M(SD) } & \multicolumn{3}{|c|}{$1.3(1.8)$} \\
\hline & \multirow{3}{*}{$\mathrm{F}(\%)$} & 0-1 Day & $25,318(29.9)$ & $59,414(70.1)$ & $84,732(64.7)$ \\
\hline & & 2-5 Days & $17,867(42.5)$ & $24,120(57.5)$ & $41,987(32.0)$ \\
\hline & & 6-7 Days & $2,330(54.7)$ & $1,930(45.3)$ & $4,260(3.3)$ \\
\hline \multicolumn{6}{|c|}{ Exercise, diet and pills used to lose weight (within the last 30 days) } \\
\hline \multirow{2}{*}{\multicolumn{2}{|c|}{ Exercises to lose weight }} & Yes & $19,856(27.8)$ & $51,444(72.2)$ & $71,300(54.0)$ \\
\hline & & No & $25879(42.7)$ & $34,734(57.3)$ & $60,613(46.0)$ \\
\hline \multirow{2}{*}{\multicolumn{2}{|c|}{ Diet to lose weight }} & Yes & $12,497(24.8)$ & $37,872(75.2)$ & $50,369(38.3)$ \\
\hline & & No & $33,061(40.7)$ & $48,068(59.3)$ & $81,129(61.7)$ \\
\hline \multirow{2}{*}{\multicolumn{2}{|c|}{ Vomit or take laxatives to lose weight }} & Yes & $404(11.5)$ & $3,114(88.5)$ & $3,518(2.7)$ \\
\hline & & No & $45,102(35.3)$ & $82,757(64.7)$ & $127,859(97.3)$ \\
\hline \multirow{2}{*}{\multicolumn{2}{|c|}{ Take pills to lose weight }} & Yes & $942(21.5)$ & $3,450(78.5)$ & $4,392(3.4)$ \\
\hline & & No & $44,421(35.0)$ & $82,176(65.0)$ & $126,597(96.6)$ \\
\hline
\end{tabular}




\section{B- Sleep Behaviors and The Body Mass Index [BMI]:}

Table 2: Overall and by Gender Distribution of Sample Sleep Behaviors (IV) and the BMI (DV).

$(\mathrm{M}=$ Mean, $\mathrm{SD}=$ Standard Deviation, $\mathrm{F}=$ Frequency, $\%=$ percentage $)$

\begin{tabular}{|c|c|c|c|c|c|}
\hline & & Characteristics & Male & Female & Overall \\
\hline Sleep Variable & in the $p$ & 7 days), Number of Days & & & \\
\hline & & M(SD) & $3.3(1.9)$ & $3.0(1.9)$ & $3.1(1.9)$ \\
\hline Enough Sleep & $\Gamma_{0}(0)$ & 0-1 Day "Poor" & $8865(30.4)$ & $20280(69.6)$ & $29145(22.1)$ \\
\hline $\begin{array}{l}\text { of having enough } \\
\text { of }\end{array}$ & $F(\%)$ & 2-5 Days "Middle" & $30633(34.8)$ & $57254(65.2)$ & $87887(66.6)$ \\
\hline sleep) & & 6-7 Days "Optimal" & $6239(42.0)$ & $8626(58.0)$ & $14865(11.3)$ \\
\hline Early Awakening & & $\mathbf{M}(\mathbf{S D})$ & $0.9(1.5)$ & $1.1(1.6)$ & $1.1(1.6)$ \\
\hline & $\mathrm{F}(\%)$ & 0-1 Day "Optimal" & $34842(36.3)$ & $61197(63.7)$ & $96039(72.9)$ \\
\hline & $F(\%)$ & 2-5 Days "Middle" & $9698(30.3)$ & $22304(69.7)$ & $32002(24.3)$ \\
\hline & & 6-7 Days "Poor" & $1113(30.6)$ & $2523(69.4)$ & $3636(2.8)$ \\
\hline Daytime & & M(SD) & $3.0(2.0)$ & $3.5(2.0)$ & $3.3(2.0)$ \\
\hline Sleepiness & $\mathrm{F}(0 / 0)$ & 0-1 Day "Optimal" & $12398(43.8)$ & $15926(56.2)$ & $28324(21.5)$ \\
\hline & $F(\%)$ & 2-5 Days "Middle" & $27496(33.5)$ & $54691(66.5)$ & $82187(62.3)$ \\
\hline & & 6-7 Days "Poor" & $5834(27.3)$ & $15527(72.7)$ & $21361(16.2)$ \\
\hline Difficulty Falling & & $\mathbf{M}(\mathbf{S D})$ & $1.4(1.9)$ & $1.6(2.0)$ & $1.6(2.0)$ \\
\hline Asleep & $E(0)$ & 0-1 Day "Optimal" & $30547(36.1)$ & $53981(63.9)$ & $84528(64.1)$ \\
\hline & $F(\%)$ & 2-5 Days "Middle" & $12140(32.3)$ & $25447(67.7)$ & $37587(28.5)$ \\
\hline & & 6-7 Days "Poor" & $3021(31.0)$ & $6697(69.0)$ & $9718(7.4)$ \\
\hline Problem with Day & & No Problem "Optimal" & $6198(46.4)$ & $7168(53.6)$ & $13366(10.1)$ \\
\hline STетіеss & & $\begin{array}{l}\text { Little to Very big problem } \\
\text { "Poor" }\end{array}$ & $39573(33.4)$ & $78990(66.6)$ & $118563(89.9)$ \\
\hline Sleep Difficulty (n & hin the & t 12 months) $\quad$ F (\%) & $10190(31.2)$ & $22517(68.8)$ & $32707(24.8)$ \\
\hline Body Mass Index & $\mathrm{MI}]), \mathrm{O}$ & rall BMI and BMI Classificat & & & \\
\hline Overal & & $\mathbf{M}(\mathbf{S D})$ & $24.8(4.8)$ & $23.9(5.2)$ & $24.2(5.1)$ \\
\hline Underweight & & $\mathrm{F}(\%)$ & $1490(22.8)$ & $5035(77.2)$ & $6525(5.0)$ \\
\hline Desired weight & & $\mathrm{F}(\%)$ & $25893(31.8)$ & $55561(68.2)$ & $81454(62.6)$ \\
\hline Overweight & & $\mathrm{F}(\%)$ & $12368(44.6)$ & $15357(55.4)$ & $27725(21.3)$ \\
\hline Overall obesity & & $15,859(11.1)$ & & & \\
\hline Class & obesity & $\mathrm{F}(\%)$ & $3659(40.5)$ & $5372(59.5)$ & $9031(7.0)$ \\
\hline Class 1 & obesity & $\mathrm{F}(\%)$ & $1241(36.4)$ & $2173(63.6)$ & $3414(2.6)$ \\
\hline Class I & obesity & $\mathrm{F}(\%)$ & $550(27.8)$ & $1431(72.2)$ & $1981(1.5)$ \\
\hline
\end{tabular}




\section{A. Differences in sleep behaviors among the different BMI classes:}

\section{Inference Analysis}

\section{Chi-square test:}

Table 3a: Frequencies and percentages [F (\%)] of different sleep behaviors among the different classes of BMI (Chi-square $<.0001)$.

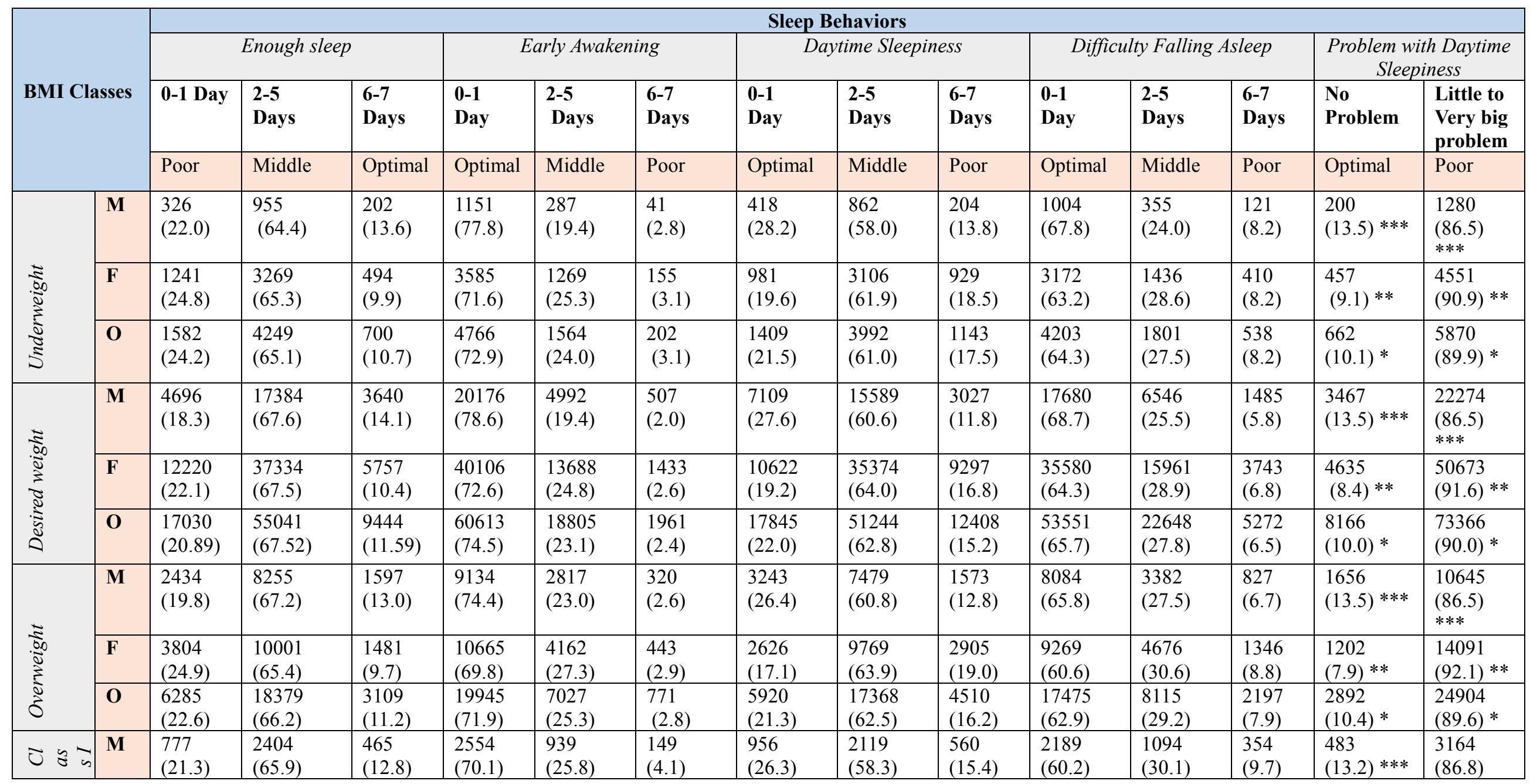




\begin{tabular}{|c|c|c|c|c|c|c|c|c|c|c|c|c|c|c|c|}
\hline & & & & & & & & & & & & & & & $* * *$ \\
\hline & $\mathbf{F}$ & $\begin{array}{l}1512 \\
(28.2)\end{array}$ & $\begin{array}{l}3404 \\
(63.6)\end{array}$ & $\begin{array}{l}437 \\
(8.2)\end{array}$ & $\begin{array}{l}3429 \\
(64.3)\end{array}$ & $\begin{array}{l}1659 \\
(31.0)\end{array}$ & $\begin{array}{l}248 \\
(4.7)\end{array}$ & $\begin{array}{l}836 \\
(15.6)\end{array}$ & $\begin{array}{l}3281 \\
(61.4)\end{array}$ & $\begin{array}{l}1229 \\
(23.0)\end{array}$ & $\begin{array}{l}2983 \\
(55.8)\end{array}$ & $\begin{array}{l}1759 \\
(32.9)\end{array}$ & $\begin{array}{l}602 \\
(11.3)\end{array}$ & $\begin{array}{l}416 \\
(7.8) * *\end{array}$ & $\begin{array}{l}4929 \\
(92.2) * *\end{array}$ \\
\hline & $\mathbf{O}$ & $\begin{array}{l}2316 \\
(25.5)\end{array}$ & $\begin{array}{l}5846 \\
(64.5)\end{array}$ & $\begin{array}{l}905 \\
(10.0)\end{array}$ & $\begin{array}{l}6026 \\
(66.6)\end{array}$ & $\begin{array}{l}2615 \\
(28.9)\end{array}$ & $\begin{array}{l}405 \\
(4.5)\end{array}$ & $\begin{array}{l}1809 \\
(20.0)\end{array}$ & $\begin{array}{l}5429 \\
(60.0)\end{array}$ & $\begin{array}{l}1811 \\
(20.0)\end{array}$ & $\begin{array}{l}5207 \\
(57.5)\end{array}$ & $\begin{array}{l}2873 \\
(31.8)\end{array}$ & $\begin{array}{l}968 \\
(10.7)\end{array}$ & $\begin{array}{l}910 \\
(10.0) *\end{array}$ & $\begin{array}{l}8151 \\
(90.0) *\end{array}$ \\
\hline \multirow{3}{*}{ 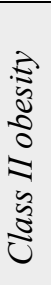 } & $\mathbf{M}$ & $\begin{array}{l}291 \\
(23.7)\end{array}$ & $\begin{array}{l}785 \\
(63.8)\end{array}$ & $\begin{array}{l}154 \\
(12.5)\end{array}$ & $\begin{array}{l}849 \\
(69 .)\end{array}$ & $\begin{array}{l}334 \\
(27.1)\end{array}$ & $\begin{array}{l}48 \\
(3.9)\end{array}$ & $\begin{array}{l}281 \\
(22.7)\end{array}$ & $\begin{array}{l}713 \\
(57.8)\end{array}$ & $\begin{array}{l}240 \\
(19.5)\end{array}$ & $\begin{array}{l}732 \\
(59.3)\end{array}$ & $\begin{array}{l}376 \\
(30.5)\end{array}$ & $\begin{array}{l}126 \\
(10.2)\end{array}$ & $\begin{array}{l}150 \\
(12.2) * * *\end{array}$ & $\begin{array}{l}1083 \\
(87.8) \\
* * *\end{array}$ \\
\hline & $\mathbf{F}$ & $\begin{array}{l}642 \\
(29.7) \\
\end{array}$ & $\begin{array}{l}1345 \\
(62.2) \\
\end{array}$ & $\begin{array}{l}175 \\
(8.1) \\
\end{array}$ & $\begin{array}{l}1387 \\
(64.2) \\
\end{array}$ & $\begin{array}{l}668 \\
(30.9) \\
\end{array}$ & $\begin{array}{l}106 \\
(4.9) \\
\end{array}$ & $\begin{array}{l}311 \\
(14.4) \\
\end{array}$ & $\begin{array}{l}1332 \\
(61.6) \\
\end{array}$ & $\begin{array}{l}519 \\
(24.0) \\
\end{array}$ & $\begin{array}{l}1207 \\
(55.8) \\
\end{array}$ & $\begin{array}{l}692 \\
(31.9) \\
\end{array}$ & $\begin{array}{l}265 \\
(12.3) \\
\end{array}$ & $\begin{array}{l}173 \\
(8.0) * *\end{array}$ & $\begin{array}{l}1990 \\
(92.0) * *\end{array}$ \\
\hline & $\mathbf{O}$ & $\begin{array}{l}947 \\
(27.6)\end{array}$ & $\begin{array}{l}2147 \\
(62.7)\end{array}$ & $\begin{array}{l}333 \\
(9.7)\end{array}$ & $\begin{array}{l}2253 \\
(65.7)\end{array}$ & $\begin{array}{l}1016 \\
(29.7)\end{array}$ & $\begin{array}{l}158 \\
(4.6)\end{array}$ & $\begin{array}{l}599 \\
(17.5)\end{array}$ & $\begin{array}{l}2063 \\
(60.1)\end{array}$ & $\begin{array}{l}769 \\
(22.4)\end{array}$ & $\begin{array}{l}1956 \\
(56.9)\end{array}$ & $\begin{array}{l}1077 \\
(31.4)\end{array}$ & $\begin{array}{l}400 \\
(11.7)\end{array}$ & $\begin{array}{l}329 \\
(9.6) *\end{array}$ & $\begin{array}{l}3101 \\
(90.4) *\end{array}$ \\
\hline \multirow{3}{*}{ 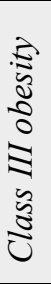 } & $\mathbf{M}$ & $\begin{array}{l}152 \\
(27.9)\end{array}$ & $\begin{array}{l}342 \\
(62.6)\end{array}$ & $\begin{array}{l}52 \\
(9.5)\end{array}$ & $\begin{array}{l}385 \\
(70.9)\end{array}$ & $\begin{array}{l}141 \\
(26.0)\end{array}$ & $\begin{array}{l}17 \\
(3.1)\end{array}$ & $\begin{array}{l}121 \\
(22.2)\end{array}$ & $\begin{array}{l}300 \\
(55.2)\end{array}$ & $\begin{array}{l}123 \\
(22.6)\end{array}$ & $\begin{array}{l}304 \\
(55.9)\end{array}$ & $\begin{array}{l}180 \\
(33.1)\end{array}$ & $\begin{array}{l}60 \\
(11.0)\end{array}$ & $\begin{array}{l}68 \\
(12.5) * * *\end{array}$ & $\begin{array}{l}477 \\
(87.5) \\
* * *\end{array}$ \\
\hline & $\mathbf{F}$ & $\begin{array}{l}434 \\
(30.4) \\
\end{array}$ & $887(62.3)$ & $\begin{array}{l}104 \\
(7.3)\end{array}$ & $\begin{array}{l}894 \\
(62.8)\end{array}$ & $\begin{array}{l}450 \\
(31.7)\end{array}$ & $\begin{array}{l}78 \\
(5.5) \\
\end{array}$ & $\begin{array}{l}179 \\
(12.5) \\
\end{array}$ & $\begin{array}{l}867 \\
(60.8)\end{array}$ & $\begin{array}{l}380 \\
(26.7) \\
\end{array}$ & $\begin{array}{l}759 \\
(53.3) \\
\end{array}$ & $\begin{array}{l}454 \\
(31.9) \\
\end{array}$ & $\begin{array}{l}211 \\
(14.8) \\
\end{array}$ & $\begin{array}{l}93 \\
(6.5) * *\end{array}$ & $\begin{array}{l}1329 \\
(93.5) * *\end{array}$ \\
\hline & $\mathbf{O}$ & $\begin{array}{l}602 \\
(30.1)\end{array}$ & $\begin{array}{l}1243 \\
(62.1)\end{array}$ & $\begin{array}{l}62.06 \\
(7.8)\end{array}$ & $\begin{array}{l}1294 \\
(64.8)\end{array}$ & $\begin{array}{l}602 \\
(30.1)\end{array}$ & $\begin{array}{l}101 \\
(5.1)\end{array}$ & $\begin{array}{l}305 \\
(15.2)\end{array}$ & $\begin{array}{l}1183 \\
(59.1)\end{array}$ & $\begin{array}{l}514 \\
(25.7)\end{array}$ & $\begin{array}{l}1074 \\
(53.7)\end{array}$ & $\begin{array}{l}648 \\
(32.4)\end{array}$ & $\begin{array}{l}278 \\
(13.9)\end{array}$ & $\begin{array}{l}165 \\
(8.3) *\end{array}$ & $\begin{array}{l}1834 \\
(91.7) *\end{array}$ \\
\hline
\end{tabular}

$(\mathrm{M}=$ Male, $\mathrm{F}=$ Female, $\mathrm{O}=$ Overall)

$*$ Chi-square $<.0 .05(=0.03) \quad * *$ Chi-square $<.0 .01 * * *$ Chi-square $=0.81$

Table 3b: Frequencies and percentages [F (\%)] of sleep difficulties (in the last 12 months) among the different classes of BMI (Chi-square $<.0001)$.

\begin{tabular}{|c|c|c|c|c|c|c|c|}
\hline \multirow[b]{2}{*}{ Sleep } & & \multicolumn{6}{|c|}{ BMI Classes } \\
\hline & & Underweight & Desired weight & Overweight & Class I obesity & Class II obesity & Class III obesity \\
\hline \multirow{3}{*}{$\begin{array}{c}\text { Sleep Difficulty } \\
\text { (in the past } 12 \text { months) }\end{array}$} & Male & $\begin{array}{c}338 \\
(23.0) \\
\end{array}$ & $\begin{array}{c}5341 \\
(20.8) \\
\end{array}$ & $\begin{array}{l}2856 \\
(23.3) \\
\end{array}$ & $\begin{array}{c}974 \\
(26.8) \\
\end{array}$ & $\begin{array}{c}351 \\
(28.5) \\
\end{array}$ & $\begin{array}{c}184 \\
(33.8) \\
\end{array}$ \\
\hline & Female & $\begin{array}{l}1298 \\
(25.9) \\
\end{array}$ & $\begin{array}{l}13531 \\
(24.5) \\
\end{array}$ & $\begin{array}{c}4248 \\
(27.8) \\
\end{array}$ & $\begin{array}{c}1714 \\
(32.1) \\
\end{array}$ & $\begin{array}{c}760 \\
(35.2) \\
\end{array}$ & $\begin{array}{c}549 \\
(38.6) \\
\end{array}$ \\
\hline & Overall & $\begin{array}{c}1652 \\
(25.3)\end{array}$ & $\begin{array}{l}19007 \\
(23.3)\end{array}$ & $\begin{array}{c}7154 \\
(25.8)\end{array}$ & $\begin{array}{l}2715 \\
(30.0)\end{array}$ & $\begin{array}{l}1126 \\
(32.9)\end{array}$ & $\begin{array}{c}748 \\
(37.5)\end{array}$ \\
\hline
\end{tabular}




\section{B. The association between the different sleep behaviors and BM I [Categories (categorical variable) and Measures (continuous variable)] (Ordinal Logistic Regression and Multivariate Linear Regression):}

Table 4a: Overall and by gender Ordinal Regression Analysis (OR (95\% CI)) for Sleep Behaviors as variables predicting Body Mass Index (BMI) Categories among college students (N=135, 874; adjusting for covariates ${ }^{\Delta}$ ).

\begin{tabular}{|c|c|}
\hline Sleep Variables & Responses: BMI Categories: OR (CI) \\
\hline \multicolumn{2}{|c|}{ Enough Sleep (in the past 7 Days) } \\
\hline Overall & $0.98(0.98-0.99) * *$ \\
\hline Male & $0.98(0.97-0.99) *$ \\
\hline Female & $0.99(0.98-1.00) \mathrm{p}=0.06$ \\
\hline \multicolumn{2}{|c|}{ Problem with Daytime Sleepiness (in the past 7 Days) Yes vs. No } \\
\hline Overall & $1.05(1.00-1.10) *$ \\
\hline Male & $1.02(0.95-1.08) \mathrm{p}=0.5$ \\
\hline Female & $1.08(1.02-1.15) *$ \\
\hline \multicolumn{2}{|c|}{ Early Awakening (in the past 7 Days) } \\
\hline Overall & $1.01(1.00-1.02) *$ \\
\hline Male & $1.02(1.00-1.03) * *$ \\
\hline Female & $1.00(1.00-1.01) \mathrm{p}=0.42$ \\
\hline \multicolumn{2}{|c|}{ Daytime Sleepiness (in the past 7 Days) } \\
\hline Overall & $1.03(1.02-1.04) * * * *$ \\
\hline Male & $1.02(1.00-1.03) * *$ \\
\hline Female & $1.04(1.03-1.05) * * * *$ \\
\hline \multicolumn{2}{|c|}{ Difficulty Falling Asleep (in the past 7 Days) } \\
\hline Overall & $1.04(1.03-1.05) * * * *$ \\
\hline Male & $1.04(1.03-1.06) * * * *$ \\
\hline Female & $1.04(1.03-1.05) * * * *$ \\
\hline \multicolumn{2}{|c|}{ Sleep Difficulties (in the past 12 Months) Yes vs. No } \\
\hline Overall & $1.05(1.01-1.08) * *$ \\
\hline Male & $1.04(0.98-1.09) \mathrm{p}=0.15$ \\
\hline Female & $1.05(1.01-1.09) *$ \\
\hline
\end{tabular}

${ }^{*} \mathrm{p}<.05 ; * * p<.01 ; * * * \mathrm{p}<.001 ; * * * * \mathrm{p}<.0001$

OR interpretation:

Less likely of being in the higher levels of BMI classes (Overweight and Obesity) $<\mathbf{1}>$ More likely of being in the higher levels of BMI classes (Overweight and Obesity)

${ }^{\Delta}$ Controlling for race, gender, age; cigarette, alcohol and marijuana use; NMUPD (Antidepressant painkillers Sedatives Stimulants); being diagnosed for ADHD (Attention Deficit Hyperactivity Disorder), insomnia, sleep 
disorders, and depression; the overall perceived stress level; and three different exercises behaviors, in the overall analysis model, and excluding gender when modelling by gender. And, when we modelled one of the sleep behavior as a predictor we controlled for the other sleep behaviors.

Table 4b: An overall and by gender Multivariate Linear Regression Analysis for the different sleep behaviors as variables predicting BMI among college students $(N=135,874)^{\Delta}$

\begin{tabular}{|l|l|l|l|}
\hline \multirow{2}{*}{$\begin{array}{l}\text { Predictors } \\
\text { Sleep Variables }\end{array}$} & \multicolumn{3}{|c|}{ Estimate (Standard Error): $B(\mathrm{SD})$} \\
\cline { 2 - 4 } & \multicolumn{1}{|c|}{ Male } & \multicolumn{1}{|c|}{ Female } & Overall \\
\hline Enough Sleep & $-0.02(0.02) \mathrm{p}=0.13$ & $-0.02(0.01) \mathrm{p}=0.09$ & $-0.02(0.01)^{*}$ \\
\hline Early Awakening & $0.04(0.02) * *$ & $0.02(0.01) \mathrm{p}=0.13$ & $0.03(0.10)^{* *}$ \\
\hline Daytime Sleepiness & $0.06(0.02) * * * *$ & $0.12(0.01) * * * *$ & $0.10(0.01)^{* * * *}$ \\
\hline Difficulty Falling Asleep & $0.11(0.01) * * * *$ & $0.11(0.01) * * * *$ & $0.11(0.01)^{* * * *}$ \\
\hline Problem with Daytime Sleepiness & $-0.01(0.08) \mathrm{p}=0.9$ & $0.22(0.07)^{* *}$ & $0.11(0.05)^{*}$ \\
\hline Sleep Difficulty & $0.07(0.07) \mathrm{p}=0.3$ & $0.16(0.05) * * *$ & $0.13(0.04) * * *$ \\
\hline
\end{tabular}

${ }^{*} \mathrm{p}<.05 ;{ }^{* *} \mathrm{p}<.01 ; * * * \mathrm{p}<.001 ; * * * * \mathrm{p}<.0001$

${ }^{\Delta}$ Controlling for race, gender, age; cigarette, alcohol and marijuana use; NMUPD (Antidepressant painkillers Sedatives Stimulants); being diagnosed for ADHD (Attention Deficit Hyperactivity Disorder), insomnia, sleep disorders, and depression; the overall perceived stress level; and three different exercises behaviors, in the overall analysis model, and excluding gender when modelling by gender. And, when we modelled one of the sleep behavior as a predictor we controlled for the other sleep behaviors. 


\section{Figures:}

I. Sample characteristics [N=135,874; Age M(SD) 22(6)]

\section{Figure 1: Demographic}

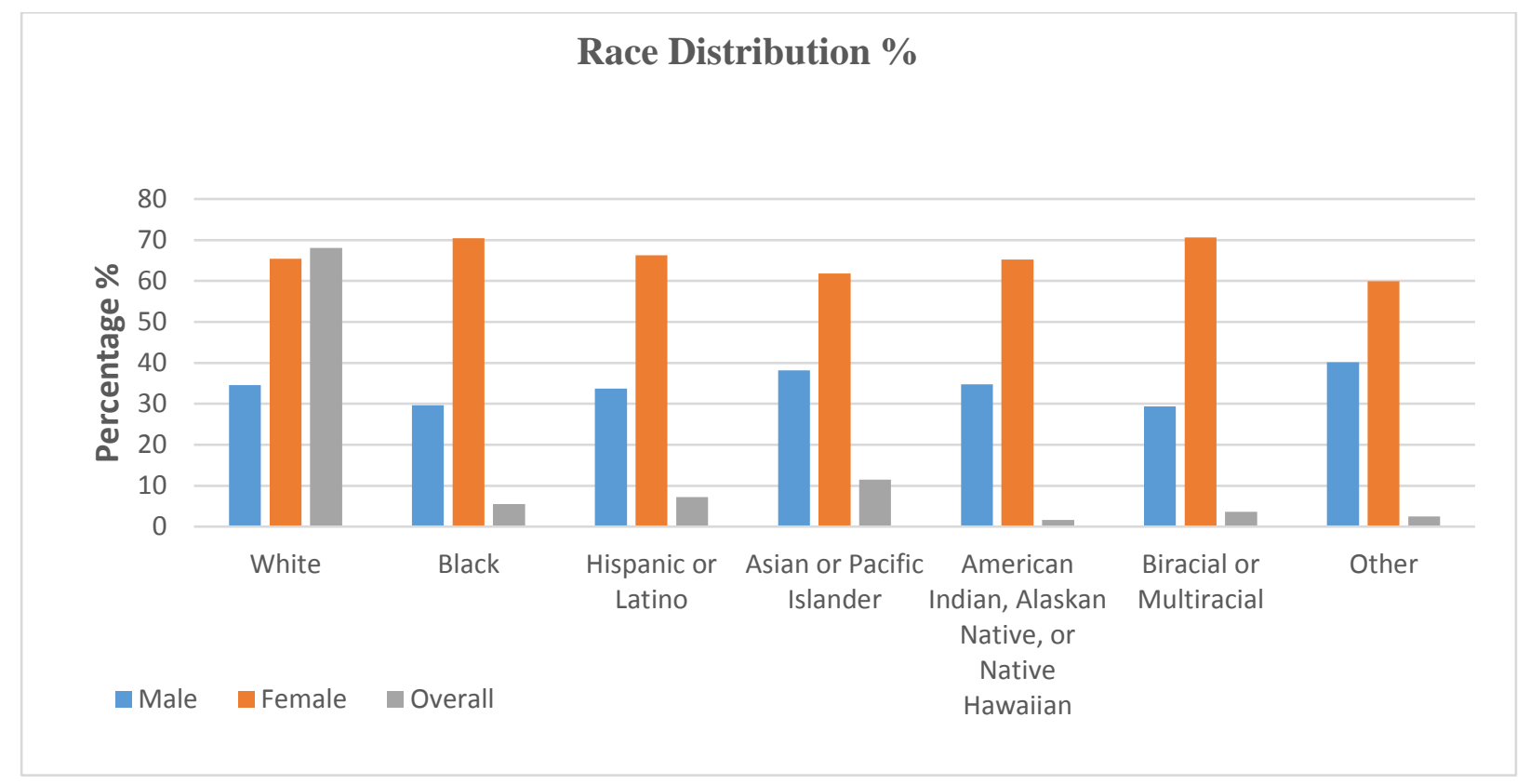

Figure ia: Race distribution by gender

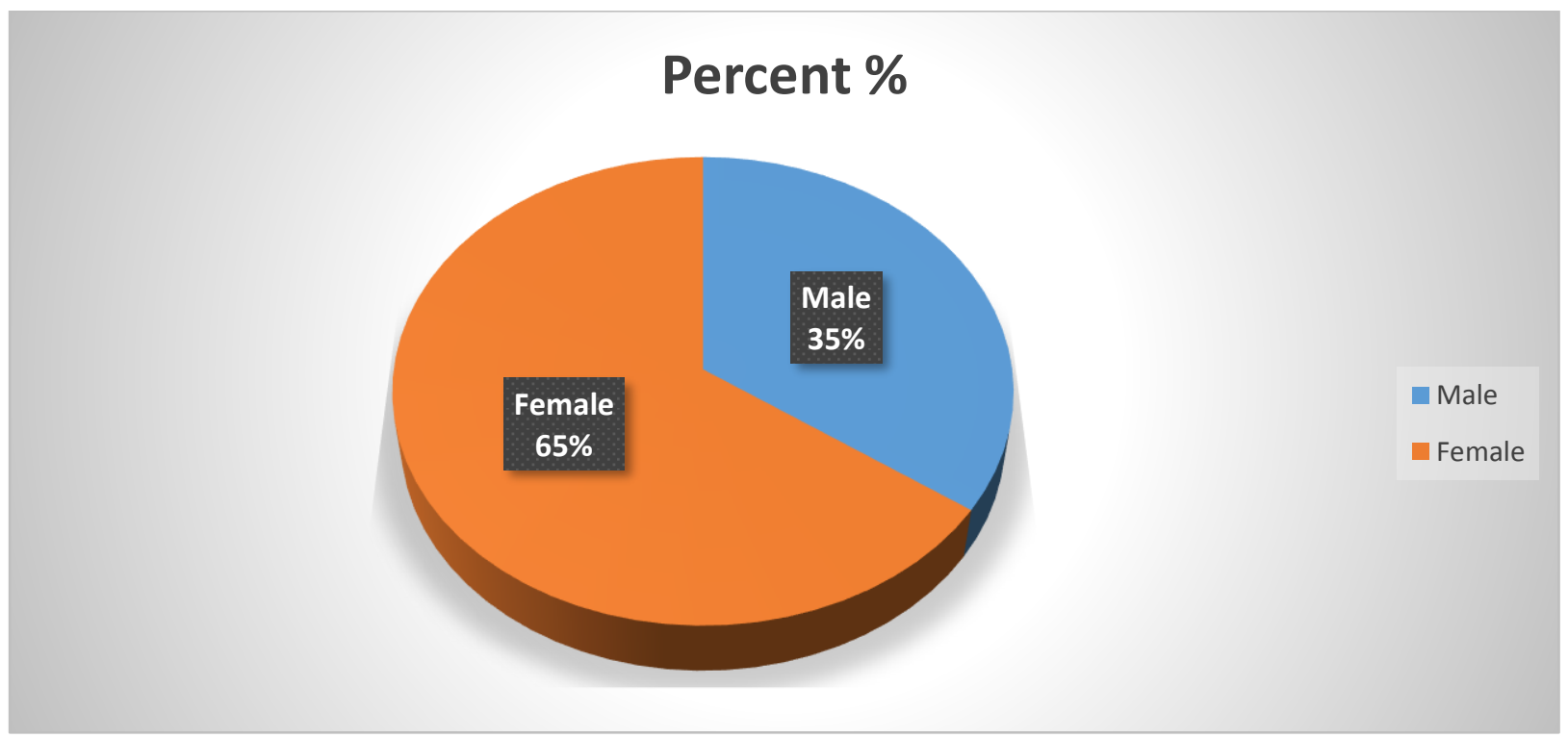

Figure iib: Gender distribution 
Figure 2: Covariates

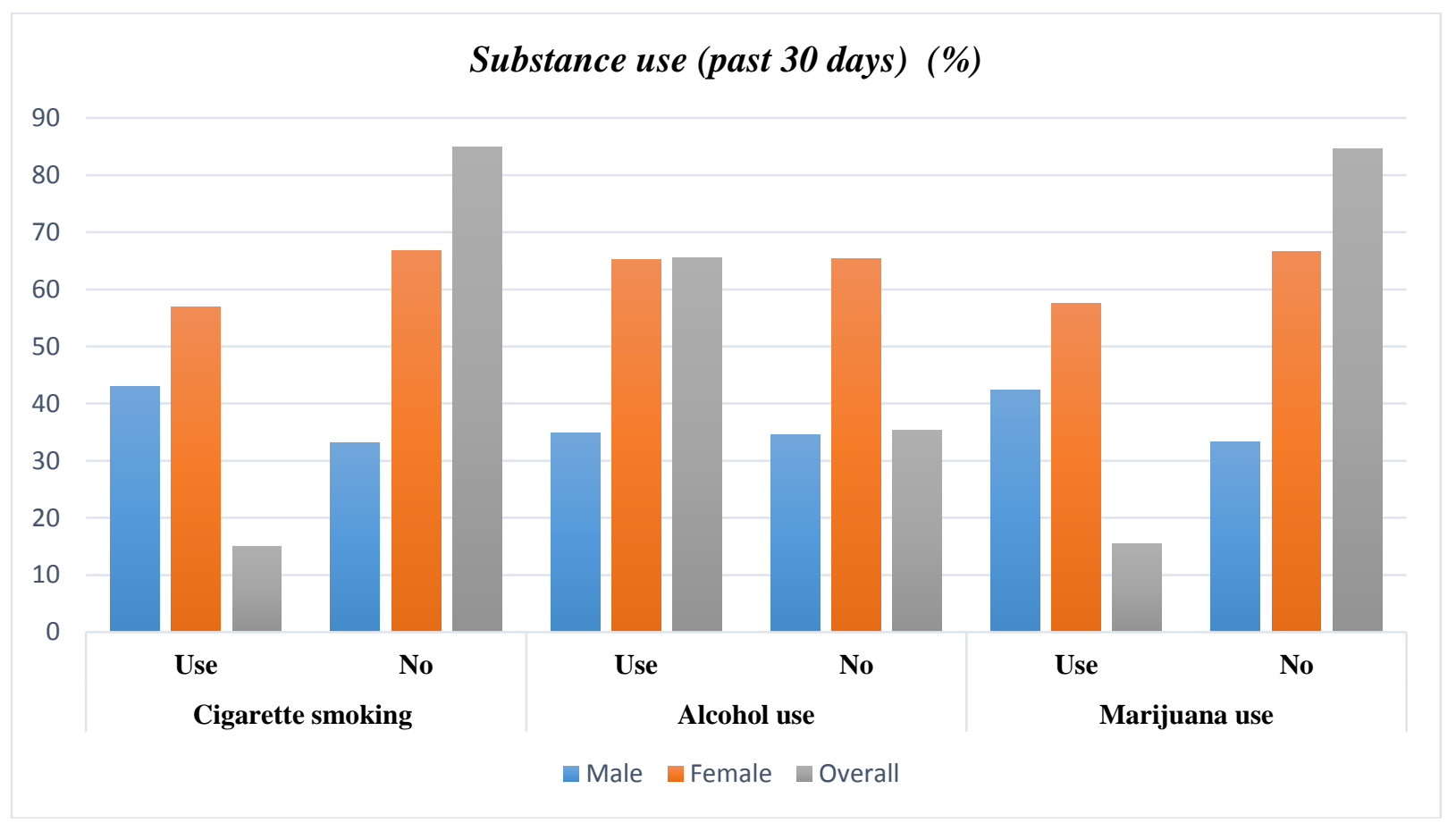

Figure 2a: Percentages of Users and Non-users of Marijuana, Alcohol, and Cigarettes among students, by gender and overall. 


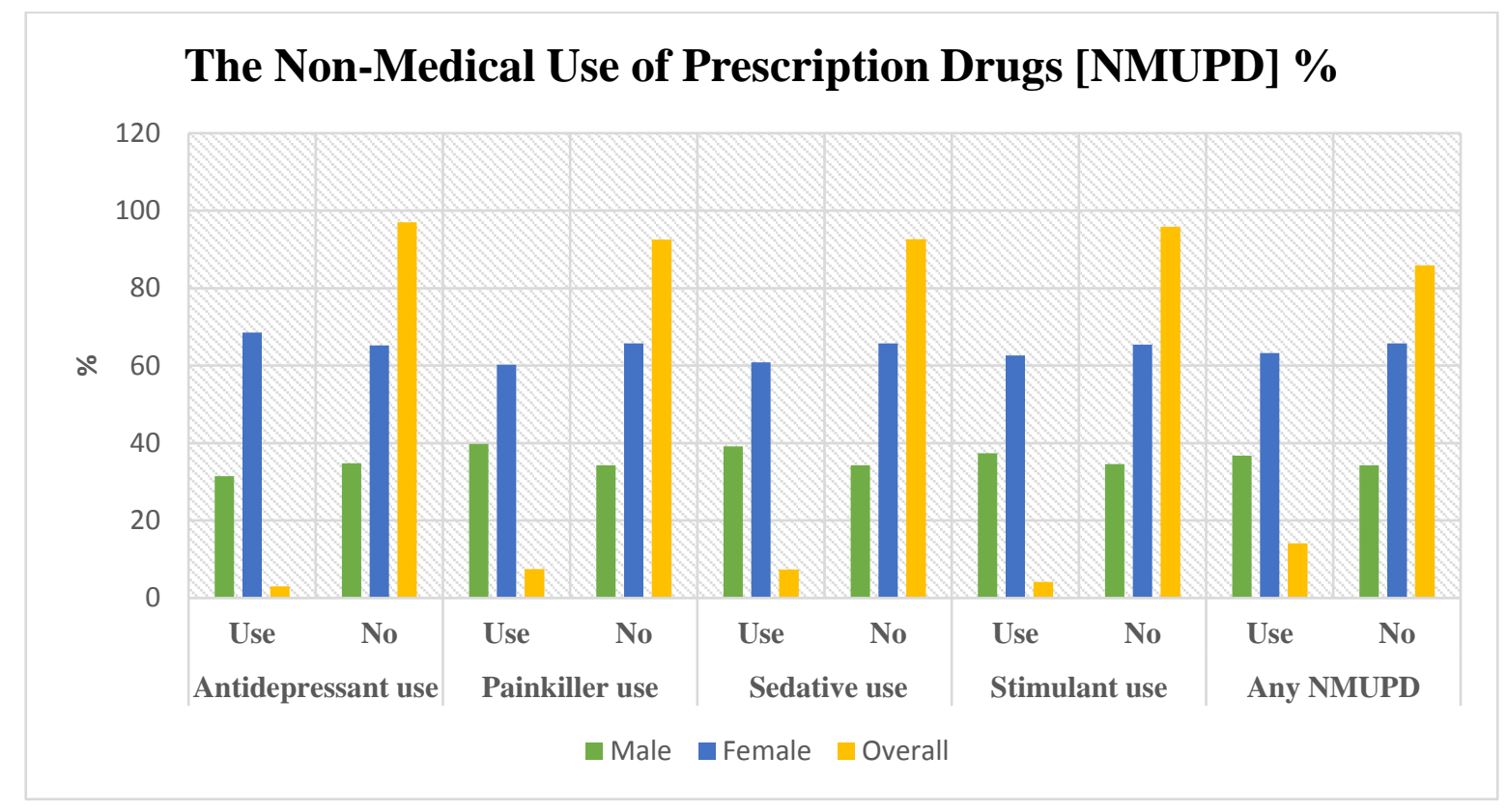

Figure 2b: Overall and by gender distribution of the non-medical use of prescription drugs [NMUPD] in the past 12 months. 


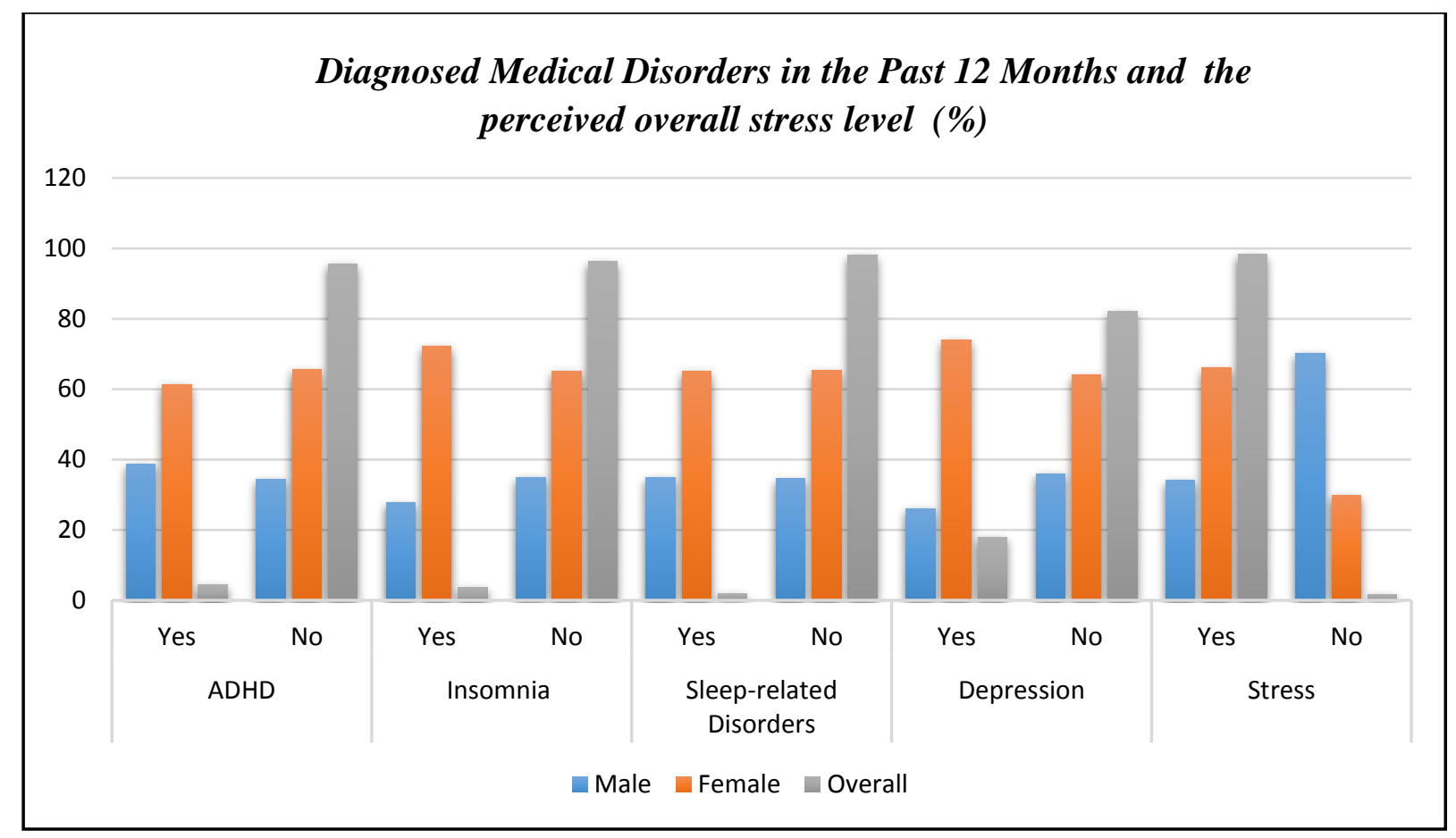

Figure 2c: The overall and by gender percentages of diagnosed medical disorders (ADHD: Attention Deficit Hyperactivity Disorder, Insomnia, Sleep-related disorders, Depresion) and the overall perceived stress level.

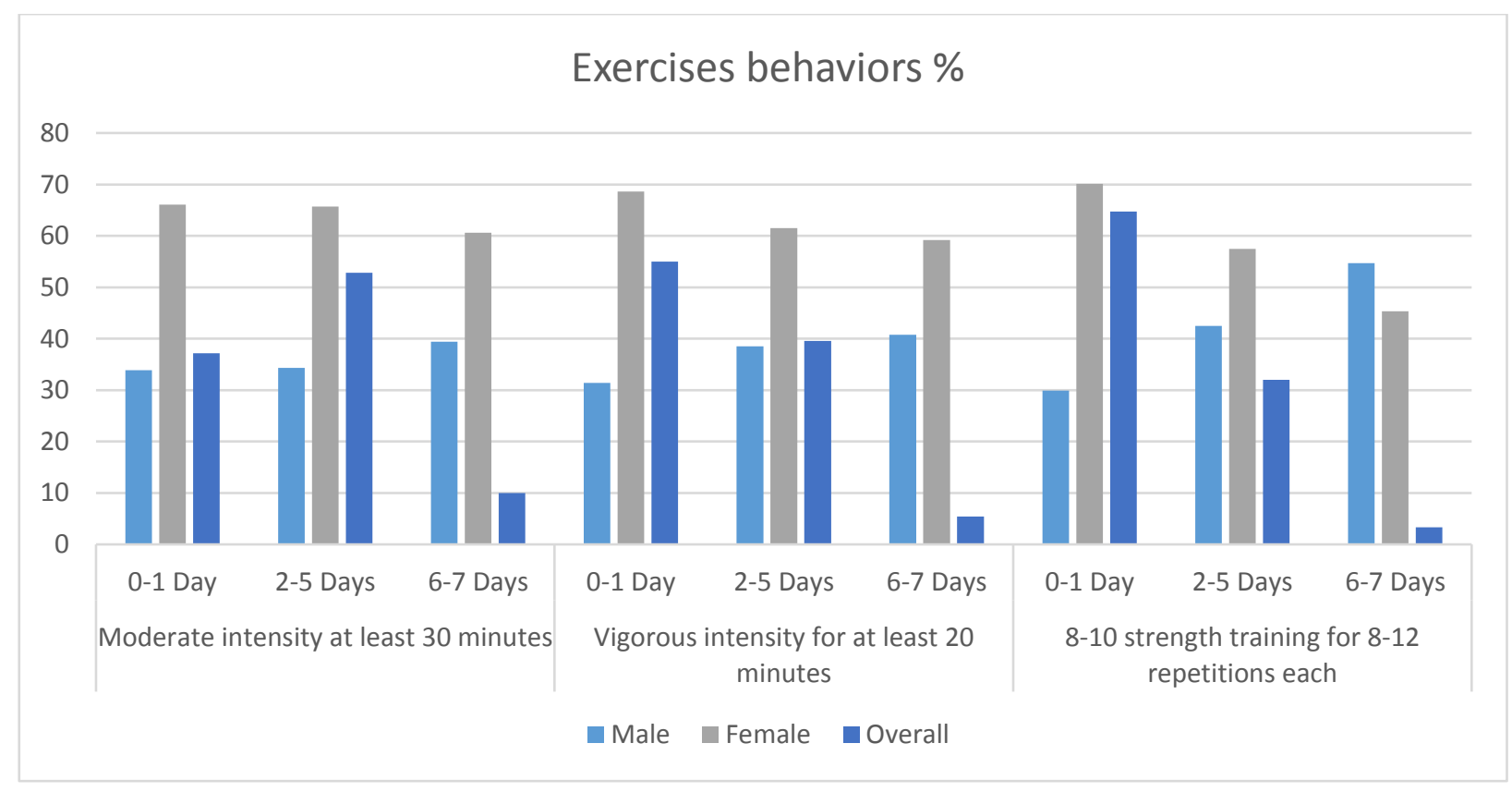

Figure 2d: The overall and by gender percentages of three different exercises behaviors in the past 7 days. 


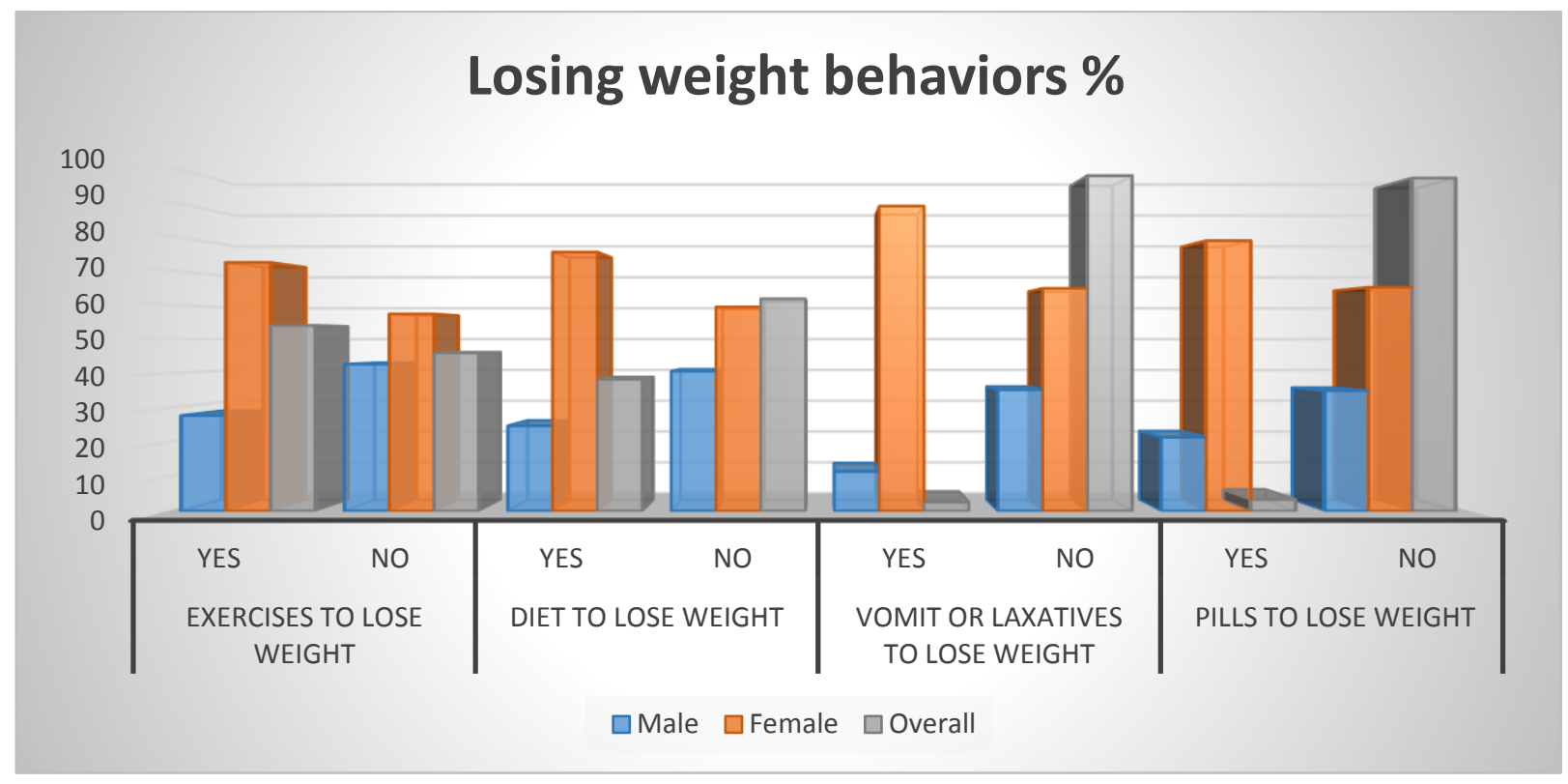

Figure 2e: The overall and by gender percentages of four different behaviors used by students to lose weight in the past 30 days. 
Figure 3: Sleep behavior and BMI distribution:

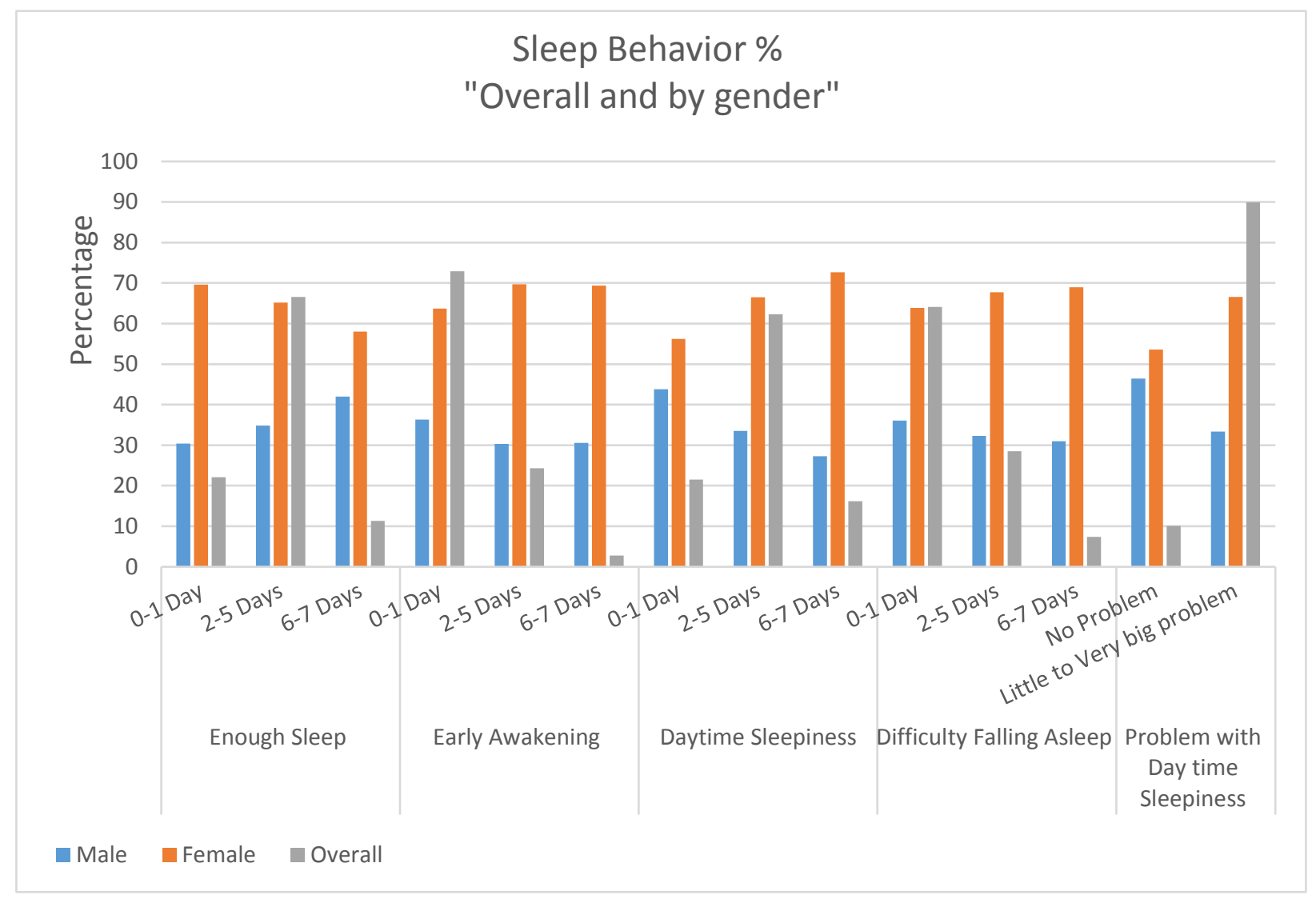

Figure 3a: Overall and by gender distribution of sample sleep behaviors (Independent variables) in the past 7 days. 


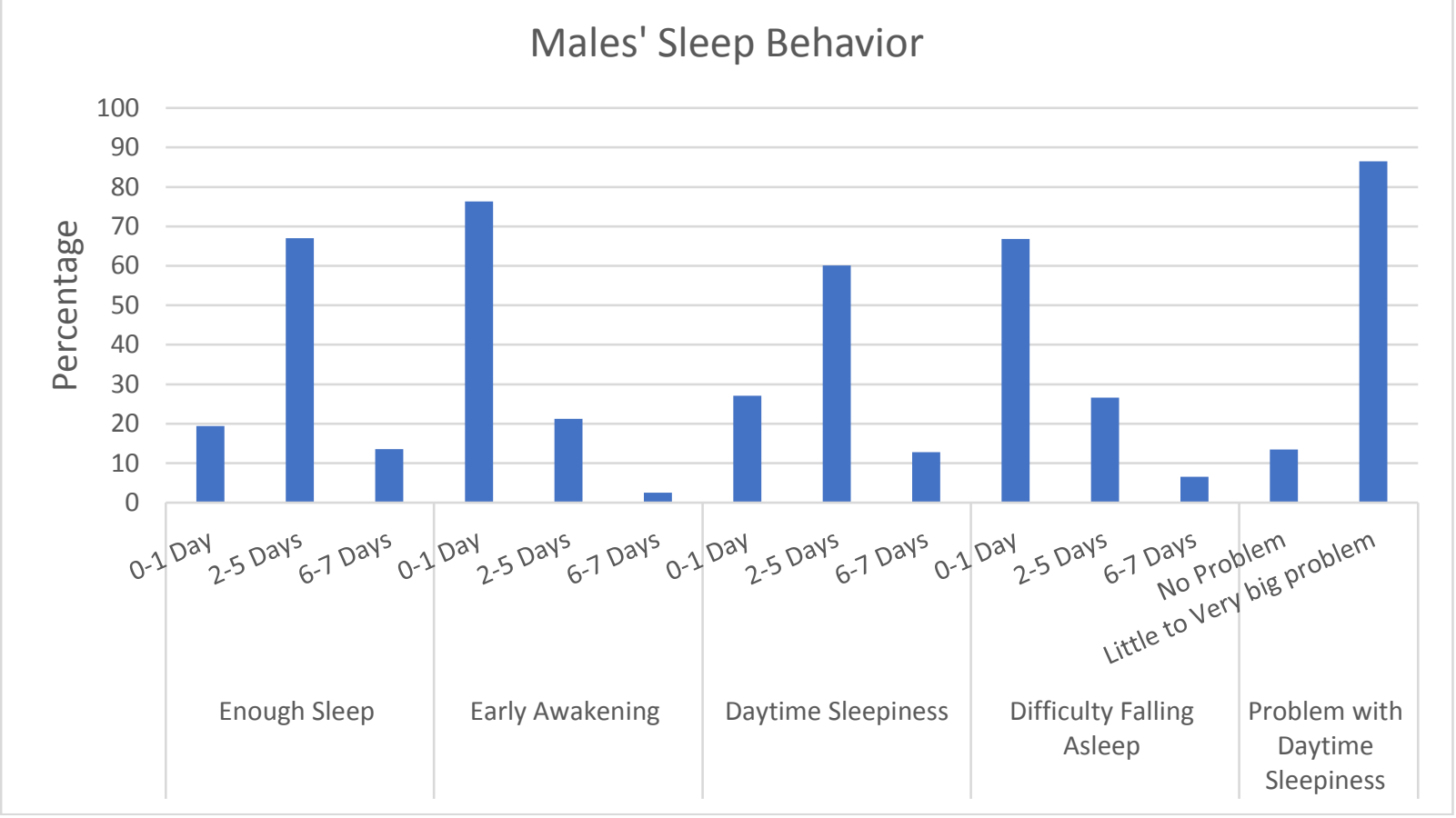

Figure 3b: Distribution of sleep behaviors (Independent variables) among male in the past 7 days.

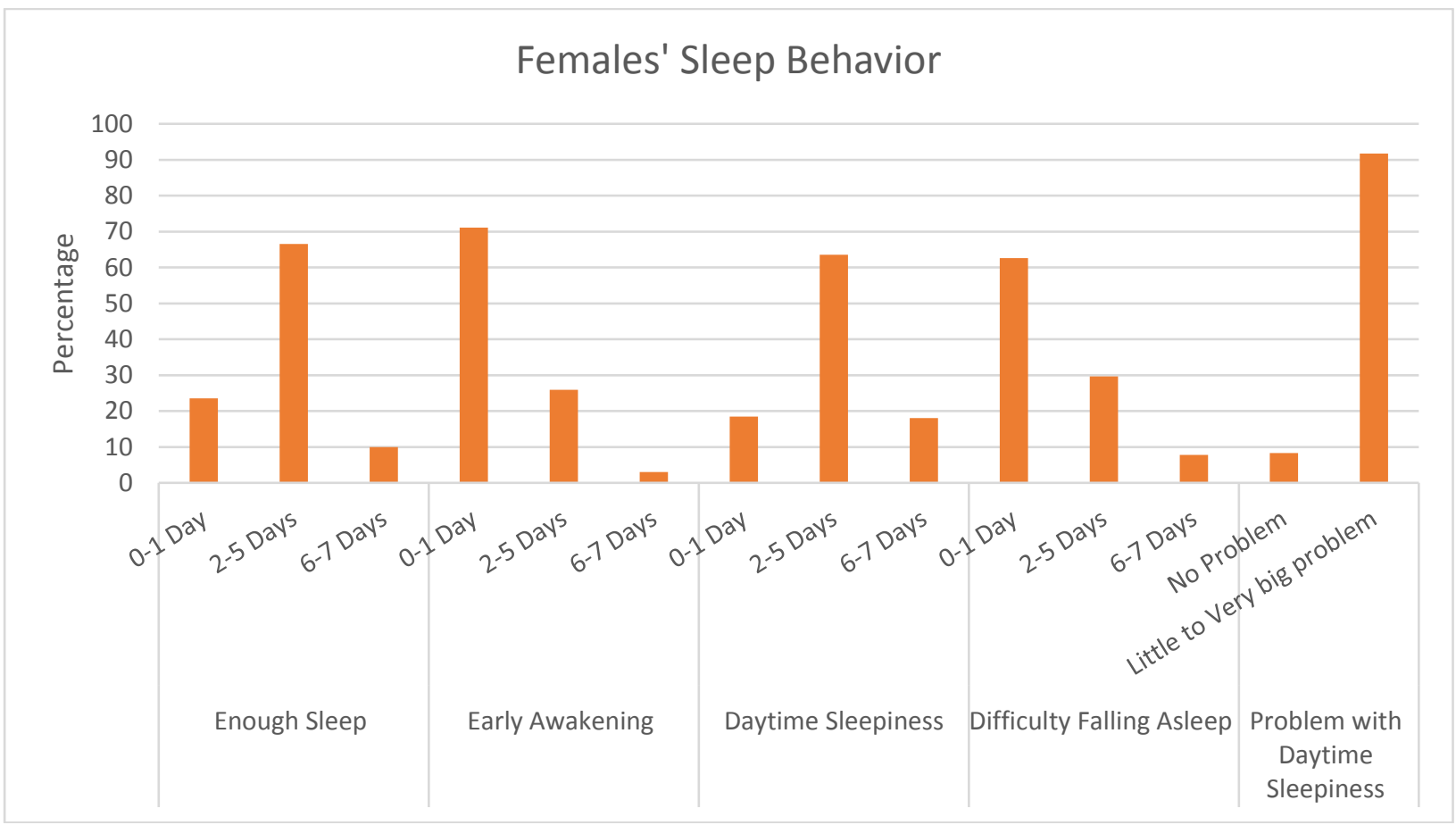

Figure 3c: Distribution of sleep behaviors (Independent variables) among female in the past 7 days. 


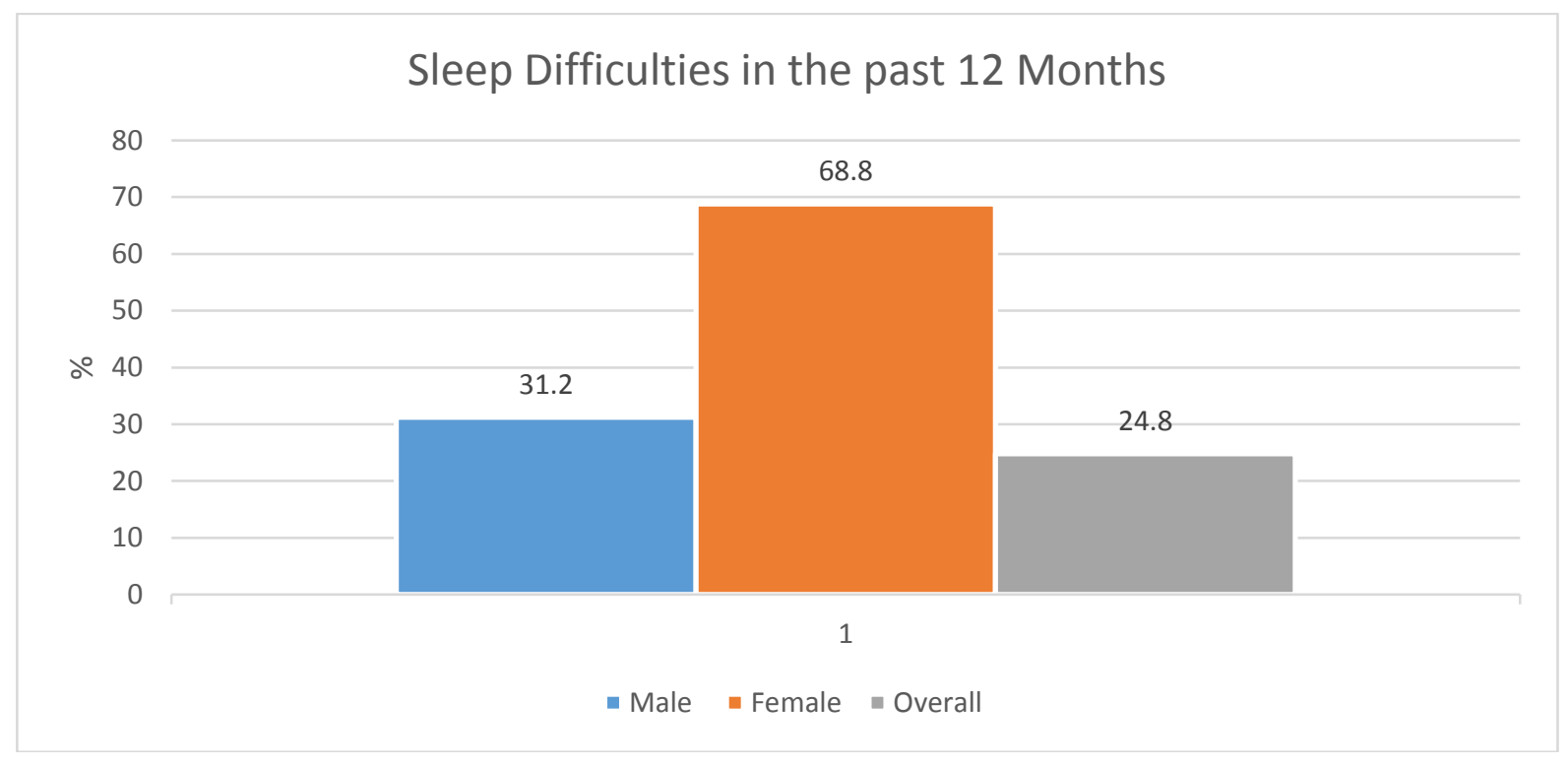

Figure 3d: An overall and by gender distribution (\%) of having sleep difficulties in the past 12 months.

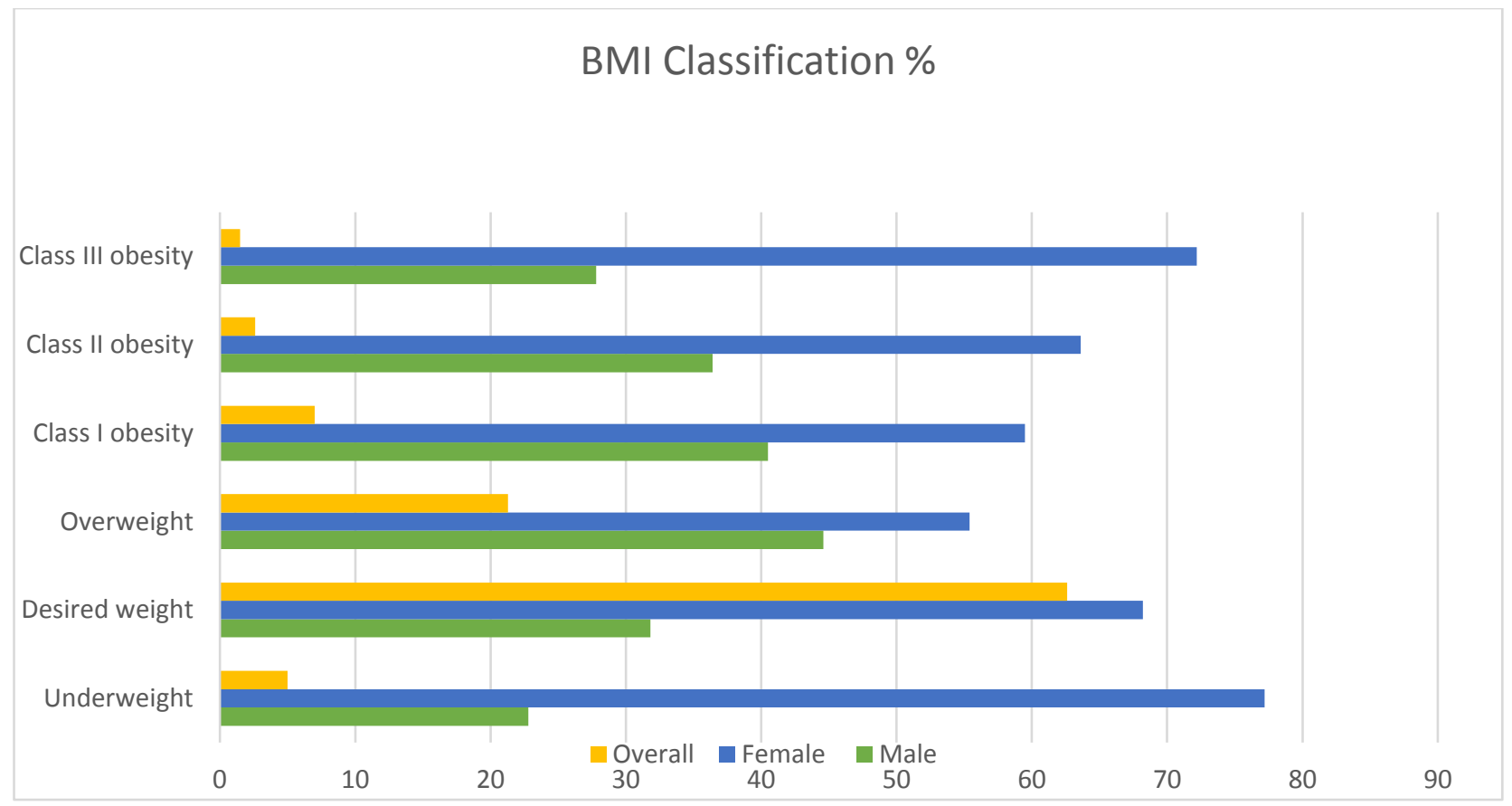

Figure 3e: Overall and by gender distribution of BMI Classifications 


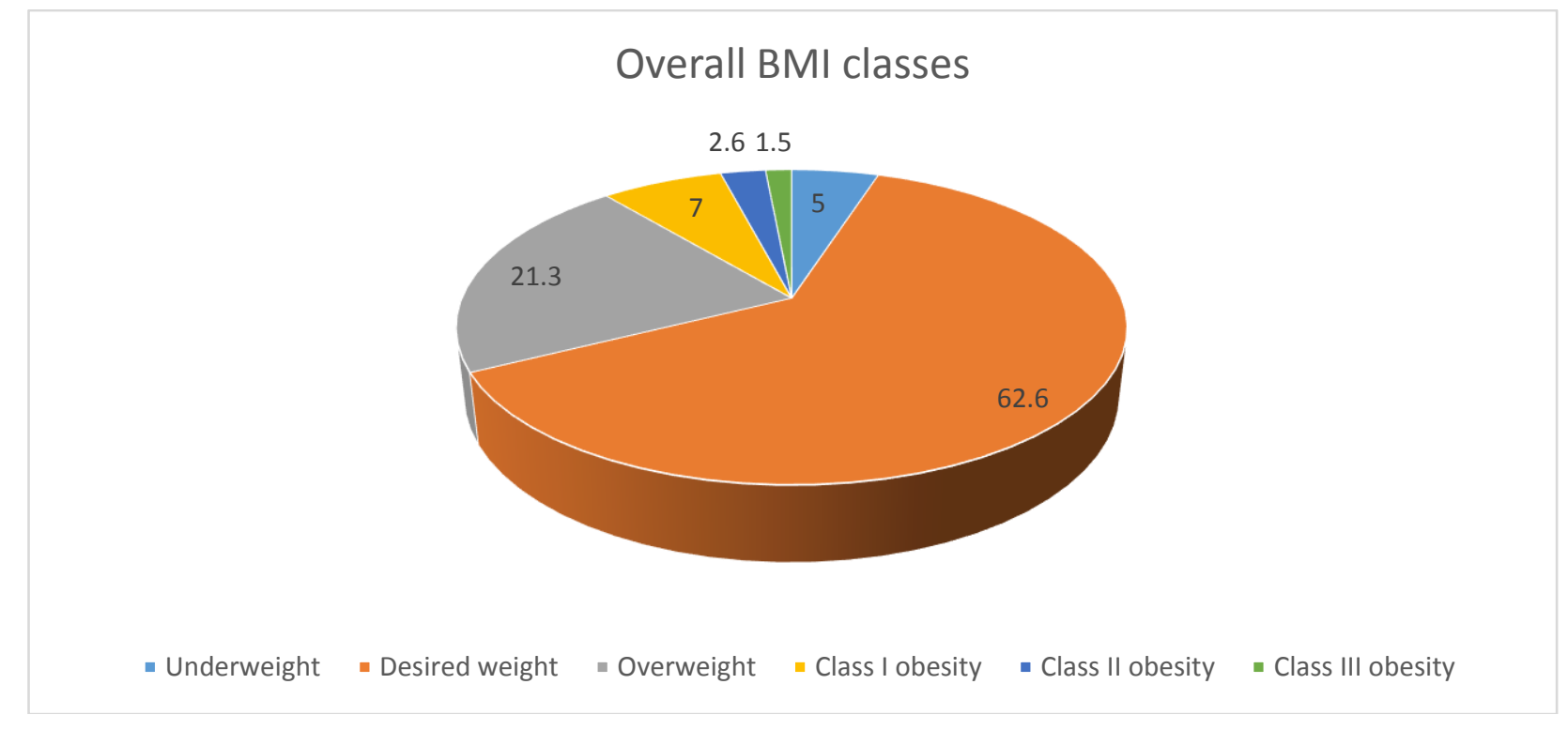

Figure 3f: Overall distribution of BMI Classifications

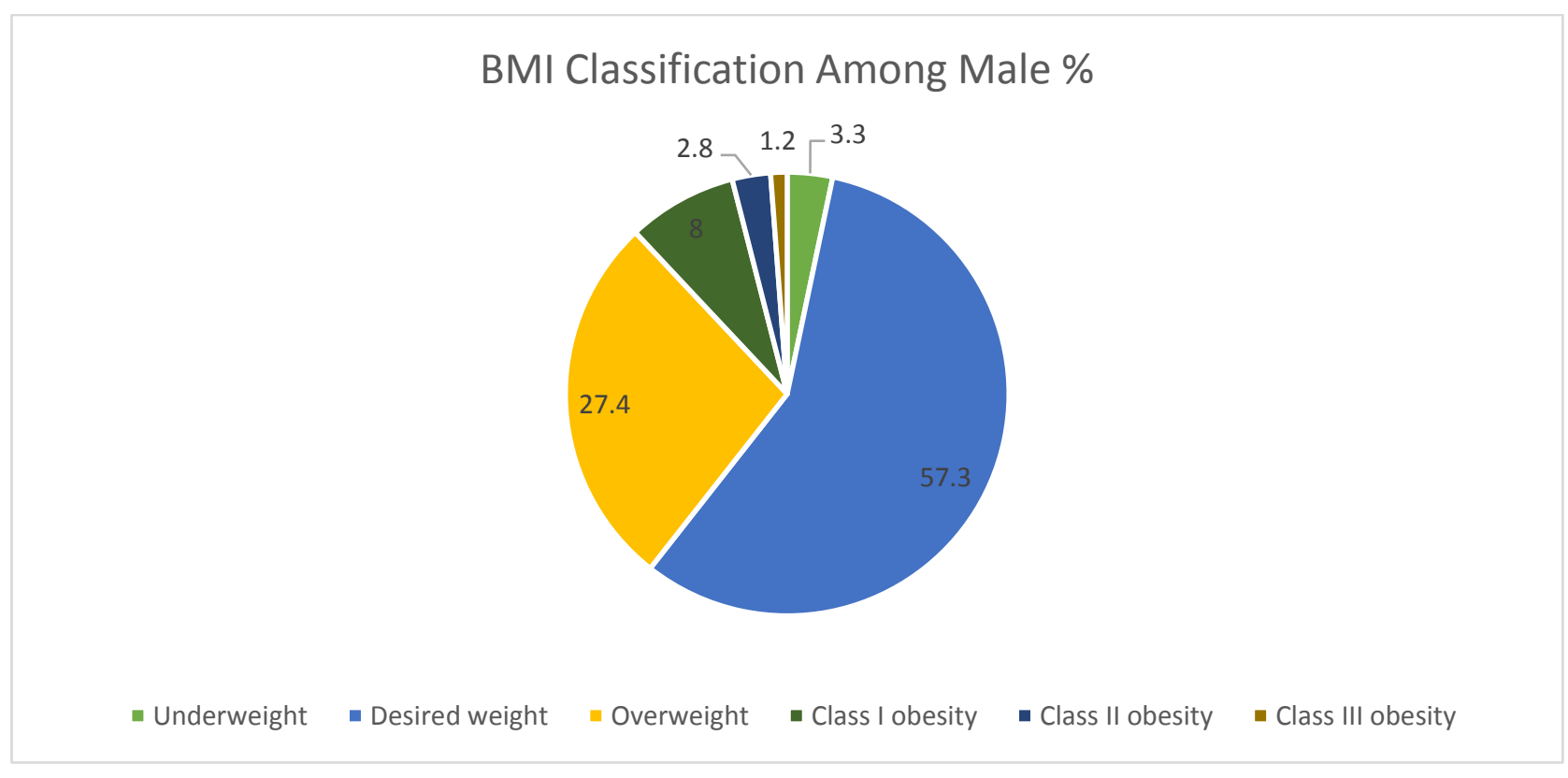

Figure 3g: Distribution of the different BMI Classifications among male 


\section{BMI Classification Among Female \%}

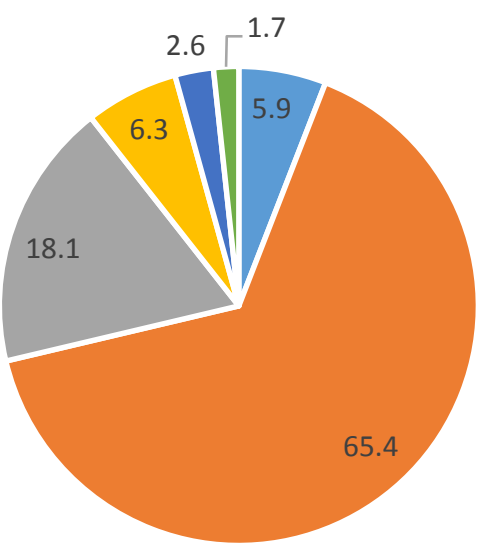

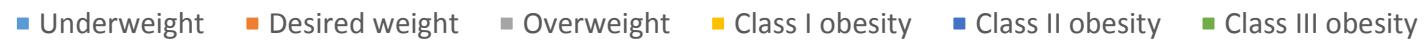

Figure 3h: Distribution of the different BMI Classifications among female 
II. Association between sleep behaviors and BMI:

Figure 4: The relationship between sleep behavior and BMI (Multivariate Ordinal Regression and Multivariate Linear Regression analysis)

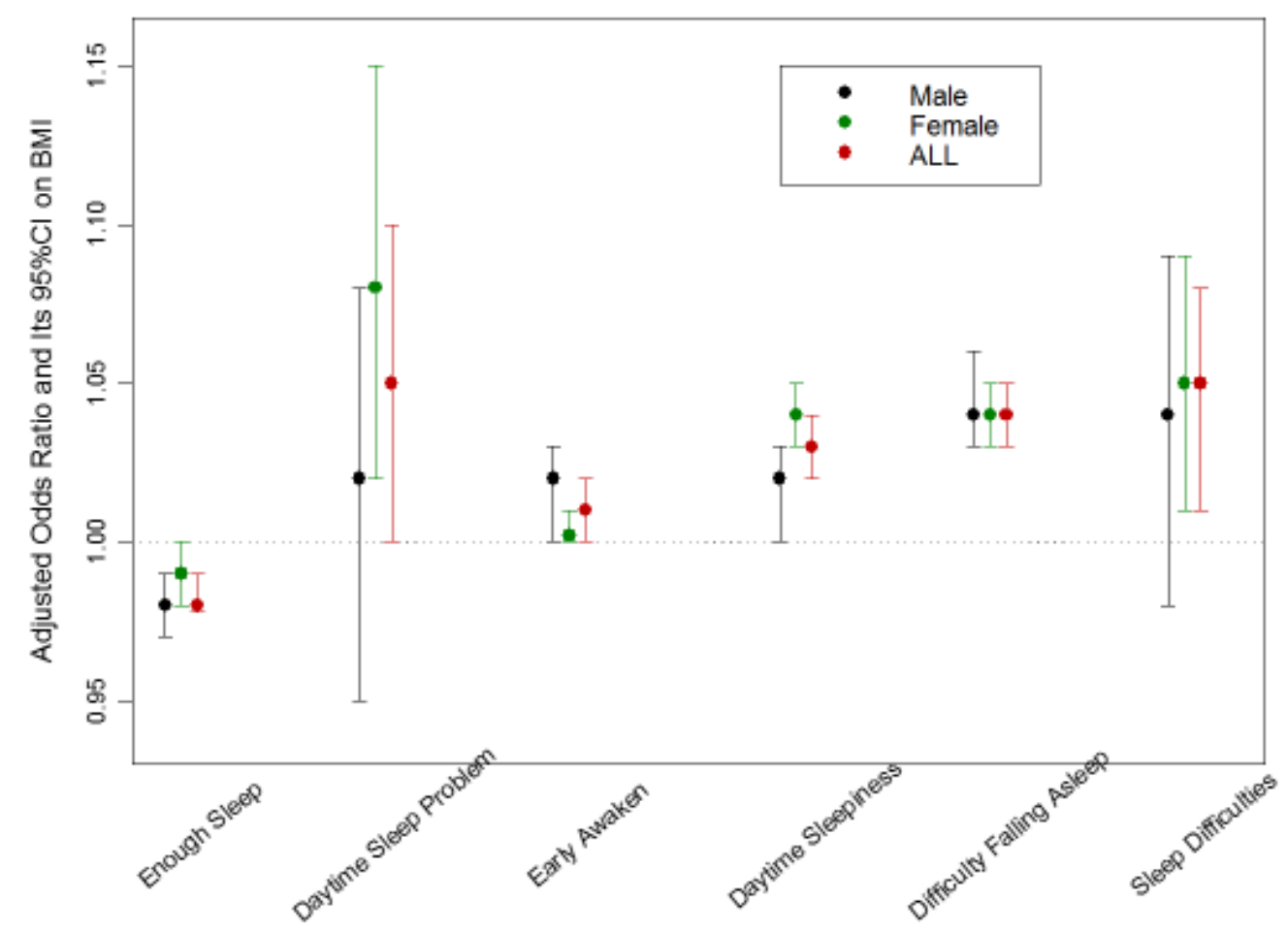

Figure 4a: Ordinal Regression Analysis (OR (95\% CI)) for the non-medical use of prescription drugs (NMUPD) as variables predicting sleep behaviors among college students 


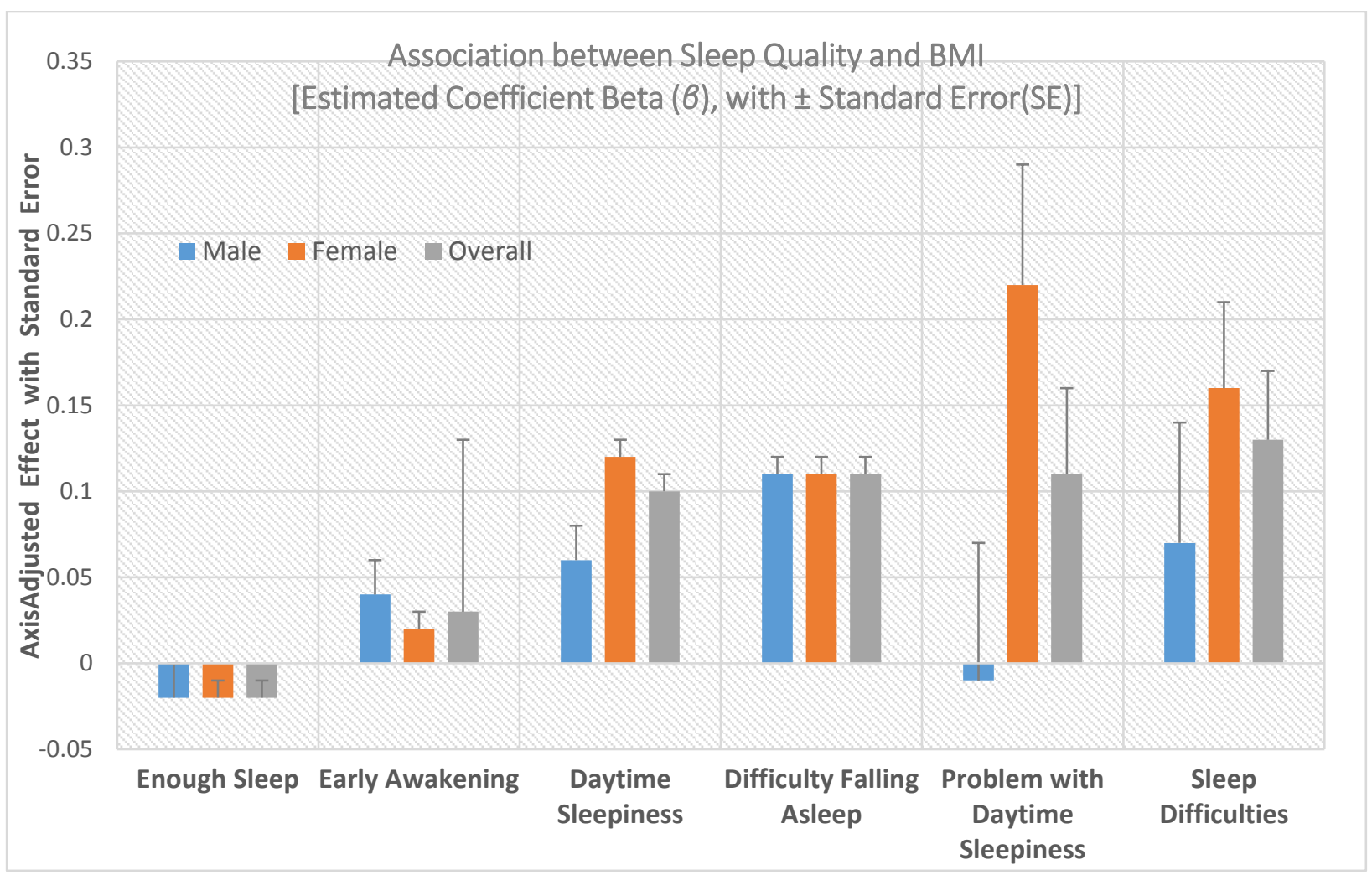

Figure 4b: Linear Regression Analysis ( $\beta(S D))$ for six sleep quality aspects as variables Predicting BMI Measures among college students (any gender). 
Appendix C

Aim3, Results' Tables and Figures 


\section{Descriptive results: sample characteristics}

Table 3: External/Covariates Variables

Table 1a: Demographic (F (\%): F=Frequency, \%= percentage)

\begin{tabular}{|c|c|c|c|c|}
\hline \multicolumn{2}{|c|}{ Characteristics } & $\begin{array}{l}\text { Male } F(\%) \\
{[n=133(38.7)]}\end{array}$ & $\begin{array}{l}\text { Female } F(\%) \\
{[n=211(61.3)]}\end{array}$ & $\begin{array}{l}\text { Overall } F(\%) \\
{[N=494(100)]}\end{array}$ \\
\hline \multicolumn{5}{|c|}{ Age $M(S D)=20(5.7)$} \\
\hline \multirow[t]{5}{*}{ Race/Ethnicity } & White & $106(35.8)$ & $190(64.2)$ & $296(87.0)$ \\
\hline & Black or African American & $10(71.4)$ & $4(28.6)$ & $14(4.0)$ \\
\hline & Asian & $6(60.0)$ & $4(40.0)$ & $10(2.9)$ \\
\hline & $\begin{array}{c}\text { American Indian, Alaskan } \\
\text { Native }\end{array}$ & $0(0.0)$ & $2(100)$ & $2(0.6)$ \\
\hline & Other & $11(57.9)$ & $8(42.1)$ & $19(5.5)$ \\
\hline \multirow[t]{4}{*}{ Year of Enrollment } & Freshman & $64(44.1)$ & $81(55.9)$ & $145(42.4)$ \\
\hline & Sophomore & $32(41.0)$ & $46(59.0)$ & $78(22.8)$ \\
\hline & Junior & $20(29)$ & $49(71.0)$ & $69(20.2)$ \\
\hline & Senior & $16(32.0)$ & $34(68.0)$ & $50(14.6)$ \\
\hline \multirow[t]{3}{*}{ Job } & Fulltime & $6(31.6)$ & $13(68.4)$ & $19(5.5)$ \\
\hline & Part-time & $38(31.7)$ & $82(68.3)$ & $120(35.0)$ \\
\hline & Unemployed & $89(43.4)$ & $116(56.6)$ & $205(59.5)$ \\
\hline \multirow[t]{5}{*}{ Annual Income } & $<\$ 10.000$ & $107(37.0)$ & $182(63.0)$ & $289(85.8)$ \\
\hline & $\$ 10.000-\$ 19.999$ & $12(57.1)$ & $9(42.9)$ & $21(6.2)$ \\
\hline & $\$ 20.0000-\$ 29.999$ & $8(50.0)$ & $8(50.0)$ & $16(4.7)$ \\
\hline & $\$ 30.000-\$ 39.999$ & $2(50.0)$ & $2(50.0)$ & $4(1.2)$ \\
\hline & $>\$ 40.000$ & $3(42.9)$ & $4(57.1)$ & $7(2.1)$ \\
\hline \multirow[t]{4}{*}{ Marital Status } & Married & $2(33.3)$ & $4(66.7)$ & $6(1.7)$ \\
\hline & separated & $1(100)$ & $0(0.0)$ & $1(0.3)$ \\
\hline & Divorced & $1(33.3)$ & $2(66.7)$ & $3(0.9)$ \\
\hline & Never married & $128(38.4)$ & $205(61.6)$ & $333(97.1)$ \\
\hline
\end{tabular}


Table 3b: Sample Distribution of Sleep-related factors (Sleep Knowledge, Bedroom Environment, \& Caffeinated Drinks) (Frequencies \& Percentages [F (\%): F= Frequency, \%= percentage; \& $\mathrm{M}(\mathrm{SD}): \mathrm{M}=$ Mean, $\mathrm{SD}=\mathrm{Standard}$ Deviation]

\begin{tabular}{|c|c|c|c|}
\hline Characteristics & Male & Female & Overall \\
\hline Sleep Knowledge* & $28.6(4.6)$ & $29.6(4.4)$ & $20.5(14.0)$ \\
\hline \multicolumn{4}{|c|}{ Bedroom Environment and sleep medication (During the past 30 days) $F(\%)$} \\
\hline $\begin{array}{l}\text { Sleepin in an uncomfortable Bedroon (e.g. Temperature, } \\
\text { light, \& noise) }\end{array}$ & $6(27.3)$ & $16(72.7)$ & $22(22.0)$ \\
\hline Sleepin in an uncomfortable Bed** & $8(50.0)$ & $8(50.0)$ & $16(4.7)$ \\
\hline $\begin{array}{l}\text { Using Sleep medication (prescribed or over the counter) } \\
\text { to help sleep. }\end{array}$ & $18(21.7)$ & $65(78.3)$ & $83(24.2)$ \\
\hline \multicolumn{4}{|l|}{ Everyday use of caffienated drinks (in the past 7 days) $F(\%)$} \\
\hline Coffee & $59(32.4)$ & $123(67.6)$ & $182(53.4)$ \\
\hline Tea & $57(36.8)$ & $98(63.2)$ & $155(45.7)$ \\
\hline Soda & $93(50.3)$ & $92(49.7)$ & $185(54.4)$ \\
\hline Energy Drinks & $33(47.8)$ & $36(52.2)$ & $69(20.2)$ \\
\hline
\end{tabular}

*Score ranges from 5-40, Higher=High/good knowledge regarding sleep and its significance. 
Table 1c: Sample Distribution of Substance use (Cigarette, Alcohol, and Marijuana) The

Nonmedical use of prescription drugs (Antidepressants, Painkiller, Stimulants, and Sedatives),

Diagnosed Medical Disorders, and the Overall Perceived Stress Level (F (\%): F=Frequency, \%= percentage)

\begin{tabular}{|c|c|c|c|}
\hline Characteristics & Male & Female & Overall \\
\hline \multicolumn{4}{|l|}{ Substance use (past 30 days) } \\
\hline Cigarette smoking & $38(41.3)$ & $54(58.7)$ & $92(27.5)$ \\
\hline Marijuana use & $51(33.6)$ & $101(66.4)$ & $152(44.4)$ \\
\hline Alcohol use (on average reported use of 1-7 days/week) & $64(32.3)$ & $134(67.7)$ & $198(58.0)$ \\
\hline \multicolumn{4}{|l|}{ The Nonmedical Use of Prescription Drugs (past 12 months) } \\
\hline Antidepressants use & $4(25.0)$ & $12(75.0)$ & $16(4.7)$ \\
\hline Painkillers use & $3(20.0)$ & $12(80.0)$ & $15(4.4)$ \\
\hline Stimulants use & $13(46.4)$ & $15(53.6)$ & $28(8.2)$ \\
\hline Sedatives use & $5(38.5)$ & $8(61.5)$ & $13(3.8)$ \\
\hline \multicolumn{4}{|c|}{ Diagnosed Medical Disorders in the Past 12 Months and the perceived overall stress level } \\
\hline Attention Deficit Hyperactivity Disordr (ADHD) & $7(31.8)$ & $15(68.2)$ & $22(6.4)$ \\
\hline Insomnia & $4(26.7)$ & $11(73.3)$ & $14(4.5)$ \\
\hline Sleep-related Disorders & $4(33.3)$ & $8(66.7)$ & $12(3.6)$ \\
\hline Depression & $10(20.0)$ & $40(80.0)$ & $50(14.6)$ \\
\hline Stress & $125(37.4)$ & $210(62.6)$ & $335(97.4)$ \\
\hline
\end{tabular}

Table 1d: Sample Distribution of Other Covariates (BMI \& BMI Classifications, Exercises, and Ways to lose weight $[\mathrm{F}(\%): \mathrm{F}=$ Frequency, $\%=$ percentage; \& $\mathrm{M}(\mathrm{SD}): \mathrm{M}=\mathrm{Mean}, \mathrm{SD}=\mathrm{Standard}$ Deviation]

\begin{tabular}{|c|c|c|c|}
\hline Characteristics & Male & Female & Overall \\
\hline \multicolumn{4}{|l|}{ Body Mass Index [BMI]), Overall BMI and BMI Classifications } \\
\hline Overall & $24.7(5.2)$ & $24.3(4.7)$ & $24.4(4.9)$ \\
\hline BMI Classifications & & $\mathbf{F}(\%)$ & \\
\hline Underweight & $13(65.0)$ & $7(35.0)$ & $20(6.0)$ \\
\hline Normal weight & $59(30.7)$ & $133(69.3)$ & $192(57.3)$ \\
\hline Overweight & $40(51.3)$ & $38(48.7)$ & $78(23.3)$ \\
\hline Obesity & $17(37.8)$ & $28(62.2)$ & $45(13.4)$ \\
\hline
\end{tabular}




\begin{tabular}{|l|c|c|c|}
\hline \multicolumn{1}{|c|}{ Exercise (in the past 7 days): Average Days [M (SD)] } & $2.1(2.1)$ & $2.1(2.1)$ \\
\hline Moderate intensity cardio or aerobic exercises for at least & $2.0(2.2)$ & $1.8(2.1)$ & $1.7(2.1)$ \\
\hline $\mathbf{3 0}$ minutes & $1.6(2.1)$ & $1.2(1.8)$ & $1.4(2.0)$ \\
\hline Vigorous intensity cardio or aerobic exercises for at least & $1.7(2.3)$ & $99(66.0)$ & $15(44.0)$ \\
\hline $\mathbf{2 0}$ minutes & & $67(71.3)$ & $94(27.5)$ \\
\hline Exercise, diet and pills used to lose weight (within the last 30 days) F (\%) & $51(34.0)$ & $3(75.0)$ & $4(1.9)$ \\
\hline Exercises to lose weight & $27(28.7)$ & $1(25.0)$ & \\
\hline Diet to lose weight &
\end{tabular}

\section{Table 2: Predictors \& Outcome Variables (Independent and Dependent Variables):}

Table 2a: The average scores of the Health Belief Model (HBM) and the Theory of Planned Behavior (TPB) Constructs: (Independent Variables) [M (SD), M= Mean, $\mathrm{SD}=$ Standard Deviation].

\begin{tabular}{|l|c|c|c|}
\hline \multicolumn{1}{|c|}{ Characteristics } & Male & Female & Overall \\
\hline HBM Constructs & $16.7(5.7)$ & $20.2(5.1)$ & $18.8(5.5)$ \\
\hline Perceived Susceptibility & $16.2(5.0)$ & $18.9(4.6)$ & $17.8(4.9)$ \\
\hline Perceived Severity & $16.1(4.3)$ & $17.8(3.2)$ & $17.2(3.8)$ \\
\hline Perceived Benefits & $12.4(4.1)$ & $13.6(3.9)$ & $13.2(4.0)$ \\
\hline Perceived Barriers & $20.8(5.0)$ & $20.6(5.2)$ & $20.6(5.1)$ \\
\hline Self-efficacy & & \\
\hline TPB Constructs & $33.4(10.3)$ & $35.0(10.3)$ & $34.4(10.4)$ \\
\hline Attitude & $15.5(5.9)$ & $16.7(5.0)$ & $16.0(5.3)$ \\
\hline Behavioral Intention & $19.9(5.9)$ & $21.9(5.3)$ & $21.1(5.5)$ \\
\hline Subjective Norms & &
\end{tabular}

Table 2b: Frequencies and the average scores of Sleep Behavior [Pittsburgh Sleep 
Quality Index (PSQI]) (Main outcome) and Health Related Quality of Life (HRQOL) (Secondary outcome) $[\mathrm{F}(\%): \mathrm{F}=$ Frequency, \%= percentage; \& M (SD): M= Mean, SD= Standard Deviation]

*Higher Score $=$ worse sleep quality: $>5$ (based on the cutoff point of the PSQI) = Poor sleep Quality

\begin{tabular}{|c|c|c|c|c|}
\hline \multicolumn{2}{|c|}{ Characteristics (during the past 30 days) } & Male & Female & Overall \\
\hline \multicolumn{5}{|l|}{ Sleep Behavior } \\
\hline Actual Sleep Hours/night & M (SD) & $6.7(1.2)$ & $6.7(1.3)$ & $6.7(1.3)$ \\
\hline \multicolumn{2}{|c|}{$\begin{array}{c}\text { Global Sleep Quality Score* } \\
\text { M(SD) }\end{array}$} & $6.5(3.1)$ & $8.0(3.4)$ & $6.2(4.2)$ \\
\hline \multirow[t]{2}{*}{ Sleep Quality } & \begin{tabular}{l|l} 
) & Good Sleep Quality \\
\end{tabular} & $55(51.4)$ & $52(48.6)$ & $107(31.0)$ \\
\hline & Poor Sleep Quality & $78(33.0)$ & $159(67.0)$ & $237(69.0)$ \\
\hline \multicolumn{5}{|l|}{ HRQOL } \\
\hline \multicolumn{2}{|c|}{$\begin{array}{l}\text { Unhealthy Days** } \\
\text { M(SD) }\end{array}$} & $8.2(8.8)$ & $13.0(10.0)$ & $10.7(9.7)$ \\
\hline \multirow[t]{5}{*}{ Self-rated Health } & Poor & $0(0.0)$ & $1(100)$ & $1(0.3)$ \\
\hline & Fair & $19(32.2)$ & $40(67.8)$ & $59(17.2)$ \\
\hline & Good & $19(45.2)$ & $23(54.8)$ & $42(12.2)$ \\
\hline & Very Good & $68(34.5)$ & $129(65.5)$ & $197(57.3)$ \\
\hline & Excellent & $27(60.0)$ & $18(40.0)$ & $45(13.0)$ \\
\hline
\end{tabular}

** Higher Score= More Unhealthy days

\section{Inferential Analysis}


Table 3: SEM and Logistic Regression

The prediction role of the HBM and the TPB of Sleep quality (SEM):

Table 3a1: Regression Weights: (all - Default model) - UNSTANDARDIZED REGRESSION

PARAMETERS Estimate $=$ regression coefficient, $S E=$ Standard Error, $C R=$ Critical Ratio, $P=$ p-value.

\begin{tabular}{|c|c|c|c|c|c|c|c|}
\hline & $\begin{array}{l}\text { Model } \\
\text { variables }\end{array}$ & & Estimate & S.E. & C.R. & $\mathrm{P}$ & Label \\
\hline HBM & $<---$ & enrollment 2 & -.201 & .130 & -1.549 & .121 & par_6 \\
\hline HBM & $<---$ & race2 & -.187 & .442 & -.422 & .673 & par_7 \\
\hline HBM & $<---$ & gender2 & 1.696 & .333 & 5.096 & $* * *$ & par_17 \\
\hline IntentionScore & $<---$ & HBM & .657 & .158 & 4.157 & $* * *$ & par_3 \\
\hline IntentionScore & $<---$ & enrollment 2 & -.335 & .278 & -1.204 & .228 & par_8 \\
\hline IntentionScore & $<---$ & race2 & -.202 & .948 & -.213 & .831 & par_10 \\
\hline IntentionScore & $<---$ & gender2 & .558 & .693 & .806 & .420 & par_18 \\
\hline GSQS & $<---$ & IntentionScore & -.253 & .036 & -7.101 & $* * *$ & par_4 \\
\hline GSQS & $<---$ & HBM & .218 & .094 & 2.314 & .021 & par_5 \\
\hline GSQS & $<---$ & enrollment 2 & -.011 & .165 & -.069 & .945 & par_9 \\
\hline GSQS & $<---$ & race2 & .345 & .561 & .615 & .539 & par_16 \\
\hline GSQS & $<---$ & gender2 & 1.509 & .409 & 3.687 & $* * *$ & par_19 \\
\hline BenefitsScore & $<---$ & HBM & 1.000 & & & & \\
\hline SeverityScore & $<---$ & HBM & 1.915 & .191 & 10.020 & $* * *$ & par_1 \\
\hline SusceptScore & $<---$ & HBM & 1.763 & .178 & 9.880 & $* * *$ & par_2 \\
\hline Unhealthydays & $<---$ & GSQS & 1.368 & .164 & 8.325 & $* * *$ & par_11 \\
\hline Unhealthydays & $<---$ & $\mathrm{HBM}$ & .808 & .267 & 3.031 & .002 & par_12 \\
\hline Unhealthydays & $<---$ & IntentionScore & -.017 & .107 & -.158 & .874 & par_13 \\
\hline Unhealthydays & $<---$ & enrollment2 & .314 & .455 & .689 & .491 & par_14 \\
\hline Unhealthydays & $<---$ & race2 & .217 & 1.549 & .140 & .889 & par_15 \\
\hline Unhealthydays & $<---$ & gender2 & 1.734 & 1.155 & 1.501 & .133 & par_20 \\
\hline
\end{tabular}


Table 3a2: Standardized Regression Weights: (all - Default model) - STANDARDIZED REGRESSON PARAMETERS

\begin{tabular}{|lll|r|}
\hline Model Variables & & & Estimate \\
\hline HBM & $<---$ & enrollment2 & -.095 \\
HBM & $<---$ & race2 & -.026 \\
HBM & $<---$ & gender2 & .350 \\
IntentionScore & $<---$ & HBM & .288 \\
IntentionScore & $<---$ & enrollment2 & -.069 \\
IntentionScore & $<---$ & race2 & -.012 \\
IntentionScore & $<---$ & gender2 & .051 \\
GSQS & $<---$ & IntentionScore & -.402 \\
GSQS & $<---$ & HBM & .152 \\
GSQS & $<---$ & enrollment2 & -.004 \\
GSQS & $<---$ & race2 & .033 \\
GSQS & $<---$ & gender2 & .217 \\
BenefitsScore & $<---$ & HBM & .619 \\
SeverityScore & $<---$ & HBM & .906 \\
SusceptScore & $<---$ & HBM & .742 \\
Unhealthydays & $<---$ & GSQS & .461 \\
Unhealthydays & $<---$ & HBM & .190 \\
Unhealthydays & $<---$ & IntentionScore & -.009 \\
Unhealthydays & $<---$ & enrollment2 & .035 \\
Unhealthydays & $<---$ & race2 & .007 \\
Unhealthydays & $<---$ & gender2 & .084 \\
\hline
\end{tabular}

Table 3a3: Squared Multiple Correlations: (all - Default model) R2 FOR PREDICTED VARIABLES AND LATENT INDICATORS

\begin{tabular}{|l|r|}
\hline & Estimate \\
\hline HBM & .121 \\
IntentionScore & .101 \\
GSQS & .196 \\
Unhealthydays & .306 \\
SusceptScore & .550 \\
SeverityScore & .821 \\
BenefitsScore & .383 \\
\hline
\end{tabular}


The association between Sleep Quality and HRQOL (Multivariate linear Regression):

Table 3b: An overall and by gender Multivariate Linear Regression Analysis of sleep Quality as variable predicting Unhealthy Days (HRQOL) $(N=494)^{\Delta}$

\begin{tabular}{|l|c|c|l|}
\hline \multirow{2}{*}{$\begin{array}{l}\text { Predictors } \\
\text { Sleep Quality }\end{array}$} & \multicolumn{3}{|c|}{$\begin{array}{c}\text { Responses: Unhealthy Days } \\
\text { Estimate (Standard Error): } B(\mathrm{SD})\end{array}$} \\
\cline { 2 - 4 } & Male $R^{2}=0.64$ & Female $R^{2}=0.49$ & Overall $R^{2}=0.50$ \\
\hline Global Sleep Quality & $0.81(0.42) \quad \mathrm{p}=0.06$ & $0.66(0.27) *$ & $0.82(0.19) * * *$ \\
\hline
\end{tabular}

${ }^{*} p, .05, * * p<.01 ; * * * p<.001 ; * * * * p<.0001$

${ }^{\Delta}$ Controlling for Demographics (race, gender, age, Job, Marital Status, and Year of Enrollment);

Substance use (cigarette, alcohol and marijuana use); NMUPD (Antidepressant painkillers Sedatives Stimulants); being diagnosed for [ADHD (Attention Deficit Hyperactivity Disorder), insomnia, sleep disorders, and depression]; the overall perceived stress level; weight and Exercises (BMI, intensity exercise, Vigorous exercise, strength Exercises, exercise to lose weight, diet to lose weight, and pills to lose weight), Sleep-related (use of sleep medication, sleeping on an uncomfortable bed, sleeping in an uncomfortable bedroom); and the everyday use of caffienated drinks (coffee, tea, soda and energy drink, in the overall analysis model, and excluding gender when modelling by gender. 
Figures:

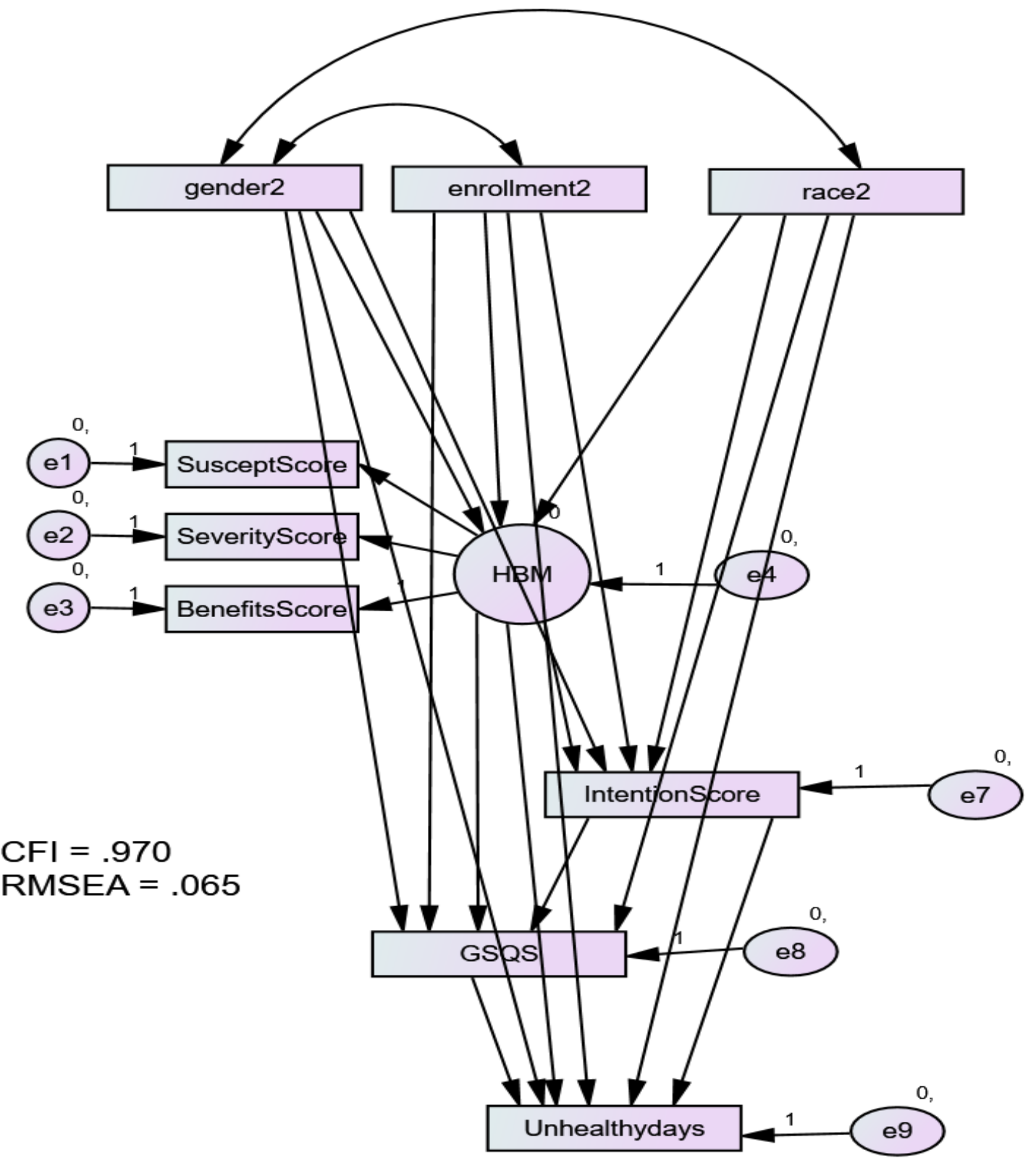

Figure 1: SEM showing thje relationships bwteen the HBM, Sleep Quality (GSQS), Behavioral Intention (InentionScore), and Unhealthydays. 


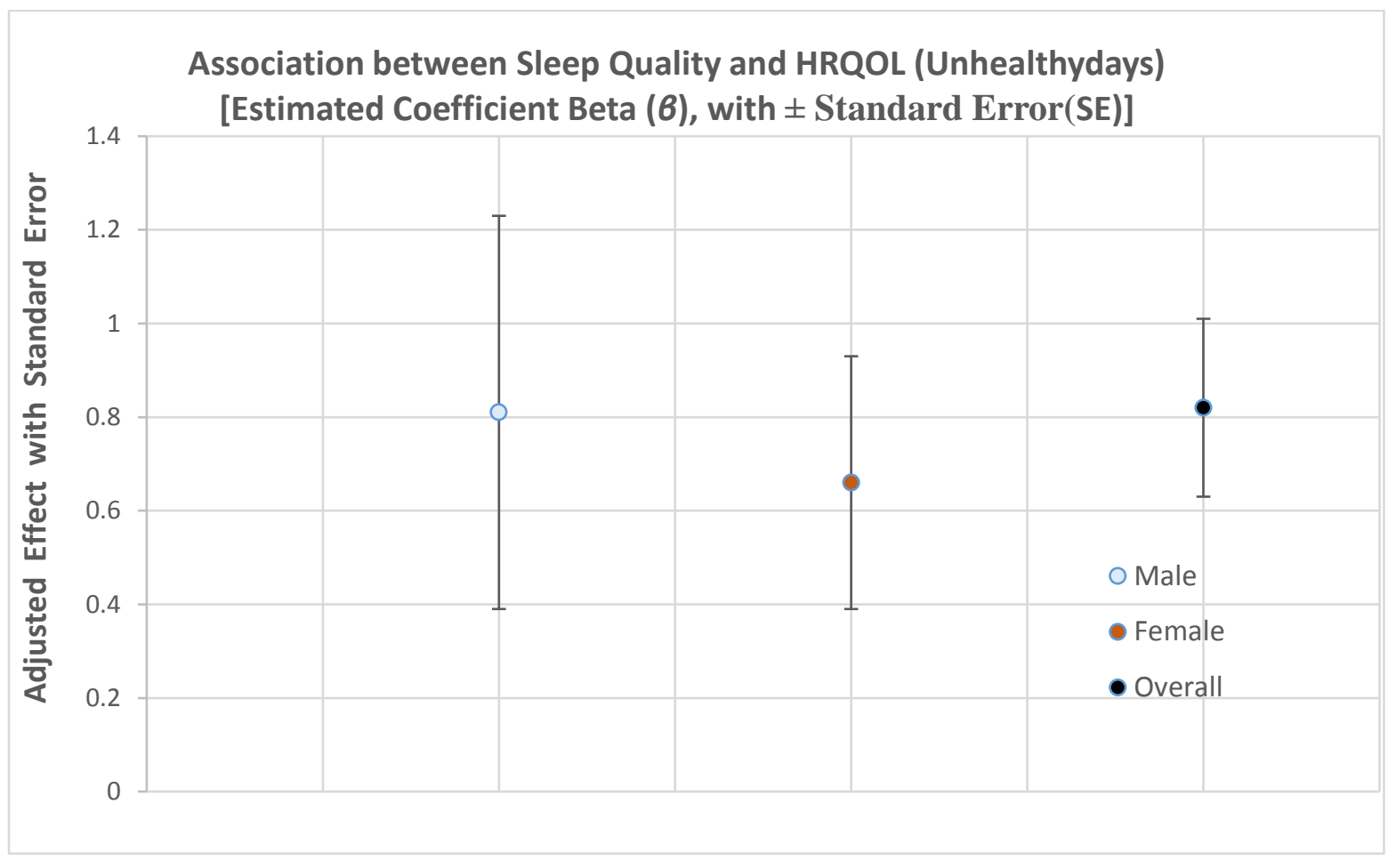

Figure 3b: By gender and overall Multivariate Linear Regression Analysis (Point estimate (SE)) for sleep quality (poor) as variables predicting unhealthy days (HRQOL). 
Appendix D

Aim3, Survey 


\section{Undergraduate Students' Sleep Behavior Survey}

1 IC Welcome..and thank you for your interest in taking the survey. The following informed consent explain what this survey is, how it works, and how your privacy will be protected. We want you to understand what are you being asked to do and what risks and benefits-if any- are associated with this survey. This will help you decide whether or not to participate. Please read the following document carefully.

\section{$1.2 \mathrm{IC}$}

\section{WestVirginiaUniversity.}

\subsection{IC Human Research Protocol Only Minimal Risk Consent Form (With HIPAA)}

\subsection{IC Principal Investigator: Keith Zullig}

Department: Social and Behavioral Sciences/ School of Public Health

Protocol Number: 1603066826

Study Title: A Theory-Based Description of Sleep Behavior and its Impact on Health-Related Quality Of Life Using the Health Belief Model and the Theory of Planned Behavior

Co-Investigator(s): Yahya Alamir

Contact Persons: If you have any questions, concerns, or complaints about this research, you can contact Dr. Keith Zullig at (304) 293- 1091 or Yahya Alamir at (681) 753-8223. For information regarding your rights as a research subject, to discuss problems, concerns, or suggestions related to the research, or would like to offer input about the research, contact the Office of Research Integrity and Compliance at (304) 293-7073. Introduction: You, have been asked to participate in this research study, which has been explained to you by Yahya Alamir. This study is being conducted by Dr. Keith Zullig and a PhD student Yahya Alamir in the Department of Social and Behavioral Sciences/ School of Public Health at West Virginia University. Purpose(s) of the Study: The project is intended to describe college students' sleep and their behavioral determinants in order to better understand sleep behavior of undergraduate college students. Additionally, we are interested in assessing the association between sleep behavior, particularly poor sleep and health related quality of life. Description of Procedures: This study involves a self-reported questionnaire that incorporates several scales measuring health-related quality of life, sleep behavior, and constructs from the Theory of Planned Behavior and Health Belief Model. You will be asked to answer questions regarding your sleep behavior, quality of life, drug and alcohol use, some medical disorders, your life at West Virginia University, and demographic information. The questionnaire will take approximately $30-$ 40 minutes for you to complete. Although it would be helpful, you do not have to answer all the 
questions. You will have the opportunity to see the questionnaire before providing your consent for this research. Discomforts: There are no known or expected risks from participating in this study, except for the mild frustration associated with answering some of the questions. Alternatives: You do not have to participate in this study. You have the right to refuse to participate and /or withdraw at any time.

1.5 IC Benefits: Information about the significance of sleep in addition guidelines to achieve healthy sleep behavior will be learned from this research, which in turn may improve general health and academic performance. Financial Considerations: You will have a chance to win one of one of 50 Panera gift cards ( $\$ 10$ value each) for your participation in this project. Confidentiality: Any information about you that is obtained as a result of your participation in this research will be kept as confidential as legally possible. Once all incentives have been dispersed, data collected through Qualtrics will be exported to Excel and then formatted for analysis in SAS. Privacy and anonymity will be maintained by replacing students' e mail addresses and other information with encoded identifiers. Then the data in Qualtrics will be destroyed, rendering all collected data anonymous. All data will be kept on a designed WVU computer located in PhD students' office, and assigned to be used only by Yahya Alamir. These files will be encrypted or password protected, and only accessed Dr. Keith Zullig, and the co-investigators, Yahya Alamir and Dr. Sijin Wen. WVU IT, keeps updated anti-virus protection on every computer, maintains upto-date versions of all software and media storage devices, and updates firewall. Computer files will be backed up and the backup data stored in a secure place physically removed from the original data. Data backups are secured and disaster recovery is routinely tested. In addition, any paper copies of research material will be kept in a locked cabinet. In any publications that result from this research, neither your name nor any information from which you might be identified will be published without your consent. 
1.6 IC HIPAA: We know that information about you and your health is private. We are dedicated to protecting the privacy of that information. Because of this promise, we must get your written authorization (permission) before we may use or disclose your protected health information or share it with others for research purposes. You can decide to sign or not to sign this authorization section. However, if you choose not to sign this authorization, you will not be able to take part in the research study. Whatever choice you make about this research study will not have an effect on your access to medical care.

Persons/Organizations Providing the Information: You as Student

Persons/Organizations Receiving the Information:

- The research team carrying out this study. This includes Dr. Keith Zullig, Yahya Alamir, and Dr. Sijin Wen.

- The members and staff of any Institutional Review Board (IRB) that oversees this research study.

- West Virginia University Office of Research Integrity and Compliance and Office of Sponsored Programs. The Following Information Will Be Used: Information from you about your sleep health, quality of life, diagnosed medical disorders, alcohol and drugs use, your life at West Virginia, knowledge regarding sleep, sleep related factors existing medical records and demographic data. The Information is Being Disclosed for the Following Reasons: Publication of study results (without identifying you). You May Cancel this Authorization at Any Time by Writing to the Principal Investigator: Keith J. Zullig, MSPH, PhD Tel: (304) 293-1091; E-mail: kzullig@hsc.wvu.edu

If you cancel this authorization, any information that was collected already for this study cannot be withdrawn. Once information is disclosed, according to this authorization, the recipient may redisclose it and then the information may no longer be protected by federal regulations. This authorization will expire at the end of the study unless you cancel it before that time (or has a specific expiration date). 
1.7 IC Voluntary Participation: Participation in this study is voluntary. You are free to withdraw your consent to participate in this study at any time. Refusal to participate or withdrawal will not affect your class standing or grades, and will involve no penalty to you. Refusal to participate or withdrawal will not affect your future care, or your employee status at West Virginia University. In the event new information becomes available that may affect your willingness to participate in this study, this information will be given to you so that you can make an informed decision about whether or not to continue your participation. You have been given the opportunity to ask questions about the research, and you have received answers concerning areas you did not understand. By clicking Agree on the next page (Electronic Consent) you are willingly consent to participate in this research. The participant has had the opportunity to have questions addressed. The participant willingly agrees to be in the study.

\section{EC Electronic Consent "Your decision":}

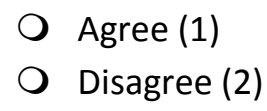

Instructions: Thank you for agreeing to take part in our study on sleep behavior! Your valuable responses to our questionnaire will help us better understand factors that influence your sleep so we learn how to promote students' sleep health. The results of this survey will help in proposing and developing health promotion programs to improve the quality of college students' sleep. The questionnaire will take you about 35 minutes to complete. Please follow the instructions for each question. If you are not comfortable answering a particular question, you may leave it blank. You may choose to stop this questionnaire at any time.

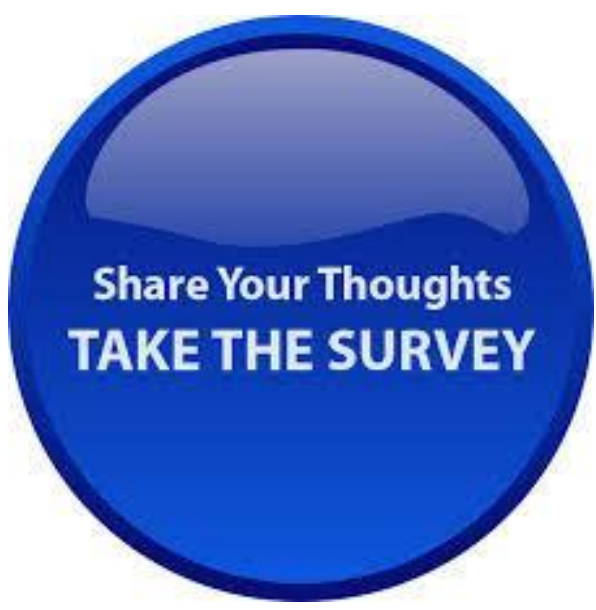


AQ1 Would you say that in general your health is:

Excellent (5)

Good (4)

Very good (3)

Fair (2)

Poor (1)

AQ2 Now thinking about your physical health, which includes physical illness and injury, for how many days during the past 30 days was your physical health not good?

Number of days (1)

None (2)

AQ3 Now thinking about your mental health, which includes stress, depression, and problem with emotion, for how many days during the past 30 days was your mental health not good?

Number of days (1)

O None (2)

AQ4 During the past 30 days, for about how many days did poor physical or mental health keep you from doing your usual activities, such as self-care, work, or recreation?

Number of days (1)

None (2)

These next questions are about physical, mental, or emotional problems or limitations you may have in your daily life

AQ5 Are you LIMITED in any way in any activities because of any impairment or health problem?

Yes (1)

O No (2) 
AQ6 What is the MAJOR impairment or health problem that limits your activities?

Arthritis/rheumatism (1)

Back or neck problem (2)

Fractures, bone/joint injury (3)

Walking problem (4)

- Lung/breathing problem (5)

- Hearing problem (6)

Eye/vision problem (7)

Heart problem (8)

Stroke problem (9)

- Hypertension/high blood pressure (10)

Diabetes (11)

- Cancer (12)

D Depression/anxiety/emotional problem (13)

口 Other impairment/problem (14)

Good Start..Keep going 
AQ7 For HOW LONG have your activities been limited because of your major impairment or health problem?

Days (4)

Weeks (3)

Months (2)

Years (1)

AQ8 Because of any impairment or health problem, do you need the help of other persons with your PERSONAL CARE needs, such as eating, bathing, dressing, or getting around the house?

Yes (1)

No (2)

AQ9 Because of any impairment or health problem, do you need the help of other persons in handling your ROUTINE needs, such as everyday household chores, doing necessary business, shopping, or getting around for other purposes?

Yes (1)

No (2)

AQ10 During the past 30 days, for about how many days did PAIN make it hard for you to do your usual activities, such as self-care, work, or recreation?

Number of days (1)

O None (2)

AQ11 During the past 30 days, for about how many days have you felt SAD, BLUE, or DEPRESSED?

Number of days (1)

None (2)

AQ12 During the past 30 days, for about how many days have you felt WORRIED, TENSE, or ANXIOUS?

Number of days (1)

O None (2) 
AQ13 During the past 30 days, for about how many days have you felt VERY HEALTHY AND FULL OF ENERGY?

Number of days (2)

O None (1)

Great....Now you will move to Sleep Health section that assesses your sleep

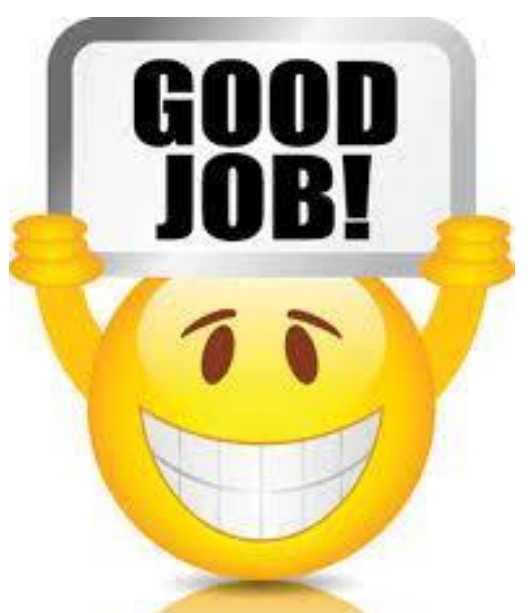


The following questions relate to your usual sleep habits during the past month only. Your answers should indicate the most accurate reply for the majority of days and nights in the past month. Please answer all questions.

BQ1 During the past month, what time have you usually gone to bed at night? BED TIME:

BQ2 During the past month, how long (in minutes) has it usually taken you to fall asleep each night? NUMBER OF MINUTES:

BQ3 During the past month, what time have you usually gotten up in the morning? GETTING UP TIME :

BQ4 During the past month, how many hours of actual sleep did you get at night? (This may be different than the number of hours you spent in bed.) HOURS OF SLEEP PER NIGHT:

For each of the remaining questions, check the one best response. Please answer all questions.

BQ5 During the past month, how often have you had trouble sleeping because you:

BQ5a Cannot get to sleep within 30 minutes

Not during the past month (4)

Less than once a week (3)

Once or twice a week (2)

O Three or more times a week (1)

BQ5b Wake up in the middle of the night or early morning

Not during the past month (4)

Less than once a week (3)

Once or twice a week (2)

O Three or more times a week (1) 
$\mathrm{BQ} 5 \mathrm{c}$ Have to get up to use the bathroom

Not during the past month (4)

Less than once a week (3)

Once or twice a week (2)

O Three or more times a week (1)

BQ5d Cannot breathe comfortably

Not during the past month (4)

Less than once a week (3)

Once or twice a week (2)

O Three or more times a week (1)

BQ5e Cough or snore loudly

Not during the past month (4)

Less than once a week (3)

Once or twice a week (2)

O Three or more times a week (1)

BQ5f Feel too cold

Not during the past month (4)

Less than once a week (3)

Once or twice a week (2)

O Three or more times a week (1)

BQ5g Feel too hot

Not during the past month (4)

Less than once a week (3)

Once or twice a week (2)

O Three or more times a week (1)

\section{BQ5h Had bad dreams}

Not during the past month (4)

Less than once a week (3)

Once or twice a week (2)

O Three or more times a week (1) 
BQ5i Have pain

Not during the past month (4)

O Less than once a week (3)

Once or twice a week (2)

O Three or more times a week (1)

BQ5j Other reason(s), please describe

BQ5j How often during the past month have you had trouble sleeping because of this?

Not during the past month (4)

Less than once a week (3)

Once or twice a week (2)

O Three or more times a week (1)

BQ6 During the past month, how would you rate your sleep quality overall?

Very good (4)

Fairly good (3)

Fairly bad (2)

Very bad (1)

BQ7 During the past month, how often have you taken medicine to help you sleep (prescribed or "over the counter")?

Not during the past month (4)

O Less than once a week (3)

Once or twice a week (2)

O Three or more times a week (1)

BQ8 During the past month, how often have you had trouble staying awake while driving, eating meals, or engaging in social activity?

Not during the past month (4)

Less than once a week (3)

Once or twice a week (2)

O Three or more times a week (1) 
BQ9 During the past month, how much of a problem has it been for you to keep up enough enthusiasm to get things done?

No problem at all (4)

O Only a very slight problem (3)

O Somewhat of a problem (2)

O A very big problem (1)

BQ10 Do you have a bed partner or roommate?

No bed partner or roommate (4)

O Partner/roommate in other room (3)

Partner in same room, but not same bed (2)

Partner in same bed (1)

You are doing Great! Keep going.....In the next two sections we are interesting in understanding your sleep behavior

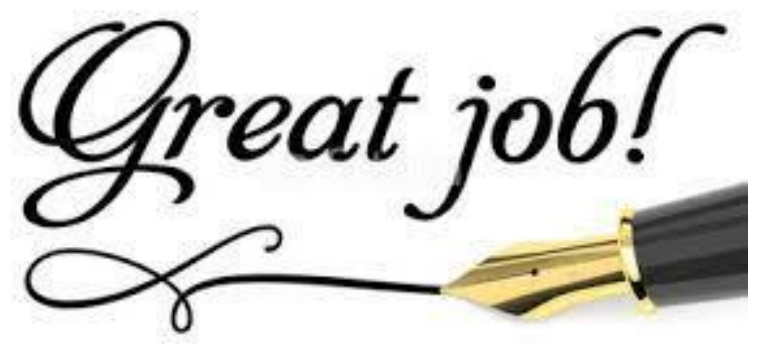

The questions in this section make use of rating scales with seven (7) answers. Please indicate where you fall on the scale. In this first set of questions, please consider how much control you feel you have over your sleeping habits

CQ1 For me, to sleep 7 to 8 hours every night is: (one being extremely not practical and 7 being extremely practical)

\begin{tabular}{|c|c|c|c|c|c|c|c|}
\hline & (1) & (2) & (3) 3 & (4) 4 & (5) & (6) 6 & (7) 8 \\
\hline $\begin{array}{c}\text { Not } \\
\text { Practical: } \\
\text { Practical } \\
\text { (CQ1_1) }\end{array}$ & 0 & 0 & 0 & 0 & 0 & 0 & 0 \\
\hline
\end{tabular}


CQ2 If I wanted to, I am sure I could sleep for 7 to 8 hours every night... (one being completely disagree and 7 being completely agree)

\begin{tabular}{|c|c|c|c|c|c|c|c|}
\hline & (1) 1 & (2) & (3) 3 & (4) 4 & $(5)^{5}$ & (6) 6 & (7) \\
\hline $\begin{array}{l}\text { Completely } \\
\text { Disagree: } \\
\text { Completely } \\
\text { Agree } \\
\text { (CQ2_1) }\end{array}$ & 0 & 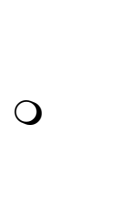 & $O$ & 0 & 0 & $O$ & 0 \\
\hline
\end{tabular}

CQ3 It is up to me whether or not I sleep 7 to 8 hours every night... (one being completely disagree and 7 being completely agree)

\begin{tabular}{|c|c|c|c|c|c|c|c|}
\hline & (1) 1 & (2) & (3) 3 & (4) 4 & (5) & (6) 6 & (7) ${ }^{7}$ \\
\hline $\begin{array}{c}\text { Completely } \\
\text { Disagree: } \\
\text { Completely } \\
\text { Agree } \\
\text { (CQ3_1) }\end{array}$ & 0 & $O$ & 0 & 0 & 0 & $\mathrm{O}$ & 0 \\
\hline
\end{tabular}

CQ4 I have complete control over my ability to sleep 7 to 8 hours every night... (one being having no control and 7 being having complete control)

\begin{tabular}{|c|c|c|c|c|c|c|c|}
\hline & (1) 1 & (2) & (3) 3 & (4) 4 & $(5)^{5}$ & (6) 6 & (7) \\
\hline $\begin{array}{c}\text { No } \\
\text { Control: } \\
\text { Complete } \\
\text { Control } \\
\text { (CQ4_1) }\end{array}$ & 0 & 0 & 0 & 0 & 0 & 0 & 0 \\
\hline
\end{tabular}


CQ5 In this question, please consider how you feel about sleeping for 7 to 8 hours every night. (one being an extremely negative option and 7 being an extremely positive option) For me to sleep 7 to 8 hours every night would be...

\begin{tabular}{|c|c|c|c|c|c|c|c|}
\hline & (1) 1 & (2) & (3) 3 & (4) 4 & (5) & (6) 6 & (7) \\
\hline $\begin{array}{c}\text { Bad: Good } \\
\text { (CQ5_1) }\end{array}$ & 0 & 0 & 0 & 0 & 0 & $\mathrm{O}$ & $\mathrm{O}$ \\
\hline $\begin{array}{l}\text { Unhealthy: } \\
\text { Healthy } \\
\text { (CQ5_2) }\end{array}$ & 0 & 0 & $\mathrm{O}$ & 0 & 0 & $O$ & 0 \\
\hline $\begin{array}{l}\text { Not } \\
\text { Refreshing: } \\
\text { Refreshing } \\
\text { (CQ5_3) }\end{array}$ & O & 0 & 0 & 0 & 0 & 0 & 0 \\
\hline $\begin{array}{l}\text { Unnecessary: } \\
\text { Necessary } \\
\text { (CQ5_4) }\end{array}$ & 0 & $\mathrm{O}$ & 0 & $\mathrm{O}$ & 0 & 0 & $\mathrm{O}$ \\
\hline $\begin{array}{c}\text { Not } \\
\text { enjoyable } \\
\text { :Enjoyable } \\
\text { (CQ5_5) }\end{array}$ & 0 & 0 & 0 & 0 & 0 & 0 & 0 \\
\hline $\begin{array}{l}\text { Detrimental } \\
\text { to my social } \\
\text { life: } \\
\text { Beneficial to } \\
\text { my social life } \\
\text { (CQ5_6) }\end{array}$ & O & 0 & 0 & 0 & 0 & 0 & 0 \\
\hline
\end{tabular}

In this next set of questions, please consider those individuals whose opinions you value most. On a scale of 1-7, with one being completely disagree and 7 being completely agree, please indicate to what extent you agree or disagree with each statement:

CQ6 My friend(s) want me to sleep 7 to 8 hours every night

\begin{tabular}{|c|c|c|c|c|c|c|c|}
\hline & (1) 1 & (2) & (3) 3 & (4) 4 & (5) & (6) & (7) \\
\hline $\begin{array}{c}\text { Completely } \\
\text { Disagree: } \\
\text { Completely } \\
\text { Agree } \\
\text { (CQ6_1) }\end{array}$ & 0 & $O$ & 0 & 0 & 0 & 0 & $\mathrm{O}$ \\
\hline
\end{tabular}


CQ7 My parent(s)/guardian(s) want me to sleep 7 to 8 hours every night

\begin{tabular}{|c|c|c|c|c|c|c|c|}
\hline & (1) & (2) & (3) & (4) & (5) & (6) & \\
\hline $\begin{array}{c}\text { Completely } \\
\text { Disagree: } \\
\text { Completely } \\
\text { Agree } \\
\text { (CQ7_1) }\end{array}$ & 0 & 0 & 0 & 0 & 0 & 0 \\
\hline
\end{tabular}

CQ8 My supervisors (e.g. professors/employers) want me to sleep 7 to 8 hours every night

\begin{tabular}{|c|c|c|c|c|c|c|c|}
\hline & (1) & (2) & (3) & (4) & (5) & (6) & (7) \\
\hline $\begin{array}{c}\text { Completely } \\
\text { Disagree: } \\
\text { Completely } \\
\text { Agree } \\
\text { (CQ8_1) }\end{array}$ & 0 & 0 & 0 & 0 & 0 & 0 & 0 \\
\hline
\end{tabular}

CQ9 Most people who are important to me want me to sleep 7 to 8 hours every night

\begin{tabular}{|c|c|c|c|c|c|c|c|}
\hline & (1) 1 & (2) ${ }^{2}$ & (3) 3 & (4) 4 & (5) & (6) ${ }^{6}$ & (7) \\
\hline $\begin{array}{c}\text { Completely } \\
\text { Disagree: } \\
\text { Completely } \\
\text { Agree } \\
\text { (CQ9_1) }\end{array}$ & 0 & 0 & 0 & 0 & 0 & 0 & 0 \\
\hline
\end{tabular}

You are about half way done 
In this set of questions, please consider your personal sleeping habits. As the same scale of 17 , one being completely disagree and 7 being completely agree, please indicate to what extent you agree or disagree with each statement:

CQ10 In the next 24 hours, I intend to sleep 7 to 8 hours at night time

\begin{tabular}{|c|c|c|c|c|c|c|c|}
\hline & (1) 1 & (2) & (3) 3 & (4) 4 & (5) & (6) & (7) \\
\hline $\begin{array}{c}\text { Completely } \\
\text { Disagree: } \\
\text { Completely } \\
\text { Agree } \\
\text { (CQ10_1) }\end{array}$ & 0 & 0 & 0 & 0 & 0 & 0 & O \\
\hline
\end{tabular}

CQ11 In the next 24 hours, I plan to sleep 7 to 8 hours at night time

\begin{tabular}{|c|c|c|c|c|c|c|c|}
\hline & (1) 1 & (2) & (3) 3 & (4) 4 & (5) & (6) 6 & (7) \\
\hline $\begin{array}{l}\text { Completely } \\
\text { Disagree: } \\
\text { Completely } \\
\text { Agree } \\
\text { (CQ11_1) }\end{array}$ & O & 0 & 0 & 0 & 0 & 0 & 0 \\
\hline
\end{tabular}

CQ12 In the next 24 hours, I will try to sleep 7 to 8 hours at night time

\begin{tabular}{|c|c|c|c|c|c|c|c|}
\hline & (1) & (2) & (3) 3 & (4) 4 & (5) & (6) & (7) \\
\hline $\begin{array}{c}\text { Completely } \\
\text { Disagree: } \\
\text { Completely } \\
\text { Agree } \\
\text { (CQ12_1) }\end{array}$ & 0 & 0 & $\mathrm{O}$ & $\mathrm{O}$ & $\mathrm{O}$ & 0 & $\mathrm{O}$ \\
\hline
\end{tabular}

In this set of questions, please consider your daily sleeping habits On a scale of 1-7, with one being always and 7 being never, please indicate how often you perform the following habits: 
CQ13 I take daytime naps lasting two or more hours

\begin{tabular}{|c|c|c|c|c|c|c|c|c|}
\hline & (1) $^{1}$ & $(2)^{2}$ & $(3)^{3}$ & $(4)^{4}$ & (5) $^{5}$ & (6) & (7) $^{7}$ \\
\hline $\begin{array}{c}\text { Always: } \\
\text { Never } \\
\text { (CQ13_1) }\end{array}$ & 0 & 0 & 0 & 0 & 0 & 0 & 0 \\
\hline
\end{tabular}

CQ14 I go to bed at different times from day to day

\begin{tabular}{|c|c|c|c|c|c|c|c|c|}
\hline & (1) $^{1}$ & $(2)$ & (3) $^{2}$ & $(4)^{4}$ & (5) $^{5}$ & (6) & (7) $^{7}$ \\
\hline $\begin{array}{c}\text { Always } \\
\text { Never } \\
\text { (CQ14_1) }\end{array}$ & 0 & 0 & 0 & 0 & 0 & 0 & 0 \\
\hline
\end{tabular}

CQ15 I get out of bed at different times from day to day

\begin{tabular}{|c|c|c|c|c|c|c|c|}
\hline & (1) $^{2}$ & $(2)^{2}$ & (3) $^{3}$ & $(4)^{4}$ & (5) $^{5}$ & (6) & (7) $^{7}$ \\
\hline $\begin{array}{c}\text { Always: } \\
\text { Never } \\
\text { (CQ15_1) }\end{array}$ & 0 & 0 & 0 & 0 & 0 & 0 & 0 \\
\hline
\end{tabular}

CQ16 I exercise to the point of sweating within one hour of going to bed

\begin{tabular}{|c|c|c|c|c|c|c|c|}
\hline & (1) 1 & (2) ${ }^{2}$ & (3) 3 & (4) 4 & (5) & (6) & (7) \\
\hline $\begin{array}{l}\text { Always: } \\
\text { Never } \\
\text { (CQ16 } 1 \text { ) }\end{array}$ & 0 & 0 & 0 & 0 & 0 & 0 & 0 \\
\hline
\end{tabular}


CQ17 I stay in bed longer than I should two or three times a week

\begin{tabular}{|c|c|c|c|c|c|c|c|}
\hline & (1) 1 & (2) & (3) 3 & (4) 4 & $(5)^{5}$ & (6) 6 & (7) \\
\hline $\begin{array}{l}\text { Always: } \\
\text { Never } \\
\text { (CQ17_1) }\end{array}$ & 0 & $O$ & 0 & 0 & $O$ & 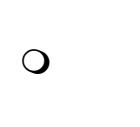 & 0 \\
\hline
\end{tabular}

CQ18 I use alcohol, tobacco, or caffeine within four hours of going to bed or after going to bed.

\begin{tabular}{|c|c|c|c|c|c|c|c|c|}
\hline & (1) $^{1}$ & $(2)$ & (3) $^{2}$ & $(4)^{4}$ & (5) $^{5}$ & (6) & (7) $^{7}$ \\
\hline $\begin{array}{c}\text { Always: } \\
\text { Never } \\
\text { (CQ18_1) }\end{array}$ & 0 & 0 & 0 & 0 & 0 & 0 & 0 \\
\hline
\end{tabular}

CQ19 I do something that may wake me up before bedtime (e.g., play video games, use the Internet, or clean)

\begin{tabular}{|c|c|c|c|c|c|c|c|}
\hline & (1) $^{2}$ & (2) $^{2}$ & (3) $^{3}$ & $(4)^{4}$ & (5) $^{5}$ & (6) & (7) $^{7}$ \\
\hline $\begin{array}{c}\text { Always: } \\
\text { Never } \\
\text { (CQ19_1) }\end{array}$ & 0 & 0 & 0 & 0 & 0 & 0 & 0 \\
\hline
\end{tabular}

CQ20 I go to bed feeling stressed, angry, upset, or nervous

\begin{tabular}{|c|c|c|c|c|c|c|c|}
\hline & (1) 1 & (2) & (3) & (4) 4 & $(5)^{5}$ & (6) & (7) \\
\hline $\begin{array}{c}\text { Always } \\
\text { :Never } \\
\text { (CQ20_1) }\end{array}$ & 0 & 0 & 0 & 0 & 0 & 0 & 0 \\
\hline
\end{tabular}


CQ21 I use my bed for things other than sleeping or sex (e.g., watch television, read, eat, or study)

\begin{tabular}{|c|c|c|c|c|c|c|c|}
\hline & (1) 1 & (2) & (3) 3 & (4) 4 & (5) & (6) 6 & (7) \\
\hline $\begin{array}{l}\text { Always: } \\
\text { Never } \\
\left(\begin{array}{ll}\text { CQ21 } & 1\end{array}\right)\end{array}$ & 0 & 0 & 0 & 0 & 0 & 0 & 0 \\
\hline
\end{tabular}

CQ22 I sleep on an uncomfortable bed (e.g., poor mattress or pillow, too much or not enough blankets)

\begin{tabular}{|c|c|c|c|c|c|c|c|c|}
\hline & (1) $^{2}$ & $(2)^{2}$ & $(3)^{3}$ & $(4)^{4}$ & (5) $^{5}$ & (6) & (7) \\
\hline $\begin{array}{c}\text { Always: } \\
\text { Never } \\
\text { (CQ22_1) }\end{array}$ & 0 & 0 & 0 & 0 & 0 & 0 & 0 \\
\hline
\end{tabular}

CQ23 I sleep in an uncomfortable bedroom (e.g., too bright, too stuffy, too hot, too cold, or too noisy)

\begin{tabular}{|c|c|c|c|c|c|c|c|}
\hline & (1) $^{1}$ & $(2)^{2}$ & (3) $^{3}$ & $(4)^{4}$ & (5) $^{5}$ & (6) & (7) $^{7}$ \\
\hline $\begin{array}{c}\text { Always: } \\
\text { Never } \\
\text { (CQ23_1) }\end{array}$ & 0 & 0 & 0 & 0 & 0 & 0 & 0 \\
\hline
\end{tabular}

CQ24 I do important work before bedtime (e.g., pay bills, schedule, or study)

\begin{tabular}{|c|c|c|c|c|c|c|c|}
\hline & (1) $^{2}$ & $(2)^{2}$ & (3) $^{3}$ & $(4)^{4}$ & (5) $^{5}$ & (6) & (7) $^{7}$ \\
\hline $\begin{array}{c}\text { Always: } \\
\text { Never } \\
\text { (CQ24_1) }\end{array}$ & 0 & 0 & 0 & 0 & 0 & 0 & 0 \\
\hline
\end{tabular}


CQ25 I think, plan, or worry when I am in bed

\begin{tabular}{|c|c|c|c|c|c|c|c|c|}
\hline & (1) $^{1}$ & $(2)^{2}$ & $(3)^{3}$ & $(4)^{4}$ & (5) $^{5}$ & (6) & (7) $^{7}$ \\
\hline $\begin{array}{c}\text { Always: } \\
\text { Never } \\
\text { (CQ25_1) }\end{array}$ & 0 & 0 & 0 & 0 & 0 & 0 & 0 \\
\hline
\end{tabular}

Keep Going....

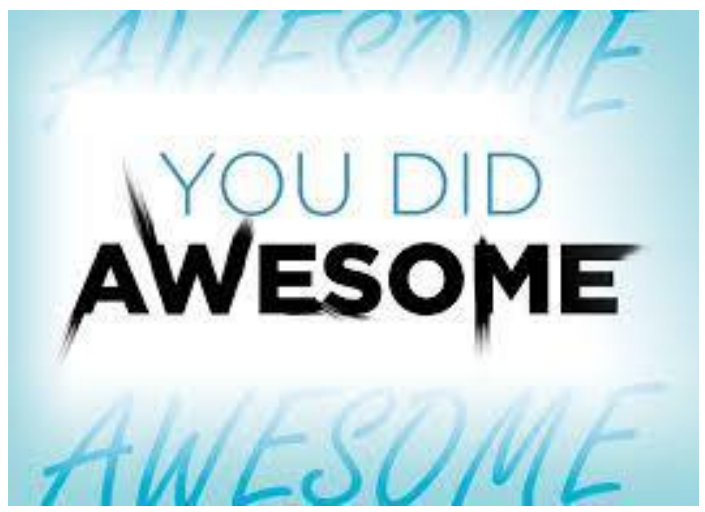


The following questions make use of rating scales with five (5) response options. Please indicate where you fall on the scale. Many different factors, such as exercise and diet, impact our overall health. As you read each question please consider how sleep alone affects the given area of health.

DQ1 If you were unable to sleep 7 to 8 continuous hours each day, how likely would you be to... (with one being not at all likely and 5 being extremely likely)

\begin{tabular}{|c|c|c|c|c|c|}
\hline & $\begin{array}{l}\text { Not } \\
\text { at all likely } \\
\text { (1) }\end{array}$ & (2) & (3) & (4) & $\begin{array}{l}\text { Extremely } \\
\text { likely (5) }\end{array}$ \\
\hline $\begin{array}{c}\text { Eventually } \\
\text { develop an illness (for } \\
\text { example, the common } \\
\text { cold). (DQ1_1) }\end{array}$ & 0 & 0 & 0 & 0 & 0 \\
\hline $\begin{array}{l}\text { Eventually } \\
\text { develop a disease (for } \\
\text { example, heart } \\
\text { disease). (DQ1_2) }\end{array}$ & 0 & 0 & O & 0 & 0 \\
\hline $\begin{array}{l}\text { Gain weight } \\
\text { over time. (DQ1_3) }\end{array}$ & 0 & 0 & 0 & 0 & 0 \\
\hline $\begin{array}{l}\text { Unintentionally } \\
\text { fall asleep while driving } \\
\text { a motor vehicle. } \\
\text { (DQ1_4) }\end{array}$ & 0 & 0 & O & 0 & 0 \\
\hline $\begin{array}{l}\text { Have a difficult } \\
\text { time focusing at work. } \\
\text { (DQ1_5) }\end{array}$ & 0 & 0 & 0 & 0 & 0 \\
\hline $\begin{array}{r}\text { Feel more } \\
\text { stressed. (DQ1_6) }\end{array}$ & 0 & 0 & 0 & 0 & 0 \\
\hline
\end{tabular}


DQ2 If you were unable to sleep 7 to 8 continuous hours each day, how much would it affect your ability to... (with one being having no affect and 5 being having major affect)

\begin{tabular}{|c|c|c|c|c|c|}
\hline & $\begin{array}{l}\text { No } \\
\text { affect (1) }\end{array}$ & (2) & (3) & (4) & $\begin{array}{l}\text { Major } \\
\text { affect (5) }\end{array}$ \\
\hline $\begin{array}{l}\text { Manage } \\
\text { stress (DQ2_1) }\end{array}$ & 0 & 0 & 0 & 0 & 0 \\
\hline $\begin{array}{l}\text { Maintain } \\
\text { good physical } \\
\text { health.. (DQ2_2) }\end{array}$ & 0 & 0 & 0 & 0 & 0 \\
\hline $\begin{array}{l}\text { Remain } \\
\text { alert while driving. } \\
\text { (DQ2_3) }\end{array}$ & 0 & 0 & 0 & 0 & 0 \\
\hline $\begin{array}{l}\text { Concentrate } \\
\text { at work. (DQ2_4) }\end{array}$ & 0 & 0 & 0 & 0 & 0 \\
\hline $\begin{array}{l}\text { Control } \\
\text { your appetite. } \\
\text { (DQ2_5) }\end{array}$ & 0 & 0 & 0 & 0 & 0 \\
\hline
\end{tabular}

DQ3 How beneficial to you is sleeping 7 to 8 continuous hours each day for...(with one being not at all beneficial and 5 being extremely beneficial)

\begin{tabular}{|c|c|c|c|c|c|}
\hline & $\begin{array}{l}\text { Not at } \\
\text { all beneficial } \\
\text { (1) }\end{array}$ & (2) & (3) & (4) & $\begin{array}{l}\text { Extremely } \\
\text { beneficial (5) }\end{array}$ \\
\hline $\begin{array}{r}\text { Your } \\
\text { energy levels. } \\
\text { (DQ3_1) }\end{array}$ & O & 0 & 0 & 0 & $\mathrm{O}$ \\
\hline $\begin{array}{l}\text { Your } \\
\text { quality of life. } \\
\text { (DQ3_2) }\end{array}$ & O & 0 & 0 & O & O \\
\hline $\begin{array}{l}\text { Your } \\
\text { work } \\
\text { productivity. } \\
\text { (DQ3_3) }\end{array}$ & 0 & 0 & 0 & 0 & 0 \\
\hline $\begin{array}{r}\text { Your } \\
\text { emotional well- } \\
\text { being (DQ3_4) }\end{array}$ & 0 & 0 & O & 0 & 0 \\
\hline
\end{tabular}


DQ4 How likely are you to not to get 7 to 8 continuous hours of sleep each day because...(with one being not at all likely and 5 being extremely likely)

\begin{tabular}{|c|c|c|c|c|c|}
\hline & $\begin{array}{l}\text { Not at } \\
\text { all likely (1) }\end{array}$ & (2) & (3) & (4) & $\begin{array}{l}\text { Extremely } \\
\text { likely (5) }\end{array}$ \\
\hline $\begin{array}{l}\text { You } \\
\text { feel pressured } \\
\text { for time. } \\
\text { (DQ4_1) }\end{array}$ & O & 0 & 0 & $\mathrm{O}$ & $\bigcirc$ \\
\hline $\begin{array}{l}\text { You } \\
\text { feel behind on } \\
\text { your work. } \\
\text { (DQ4_2) }\end{array}$ & O & 0 & 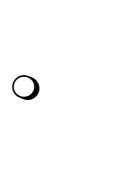 & 0 & 0 \\
\hline $\begin{array}{l}\quad \text { Getting } \\
7 \text { to } 8 \text { hours of } \\
\text { continuous } \\
\text { sleep each day } \\
\text { would require } \\
\text { starting a new } \\
\text { habit, which is } \\
\text { difficult. } \\
\text { (DQ4_3) }\end{array}$ & 0 & 0 & 0 & 0 & 0 \\
\hline $\begin{array}{l}\text { It is } \\
\text { easier to } \\
\text { sacrifice sleep } \\
\text { during work } \\
\text { days and make } \\
\text { up for lost sleep } \\
\text { during days off } \\
\text { work (for } \\
\text { example, the } \\
\text { weekend).. } \\
\text { (DQ4_4) }\end{array}$ & 0 & 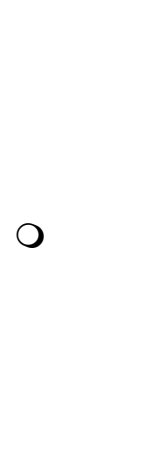 & 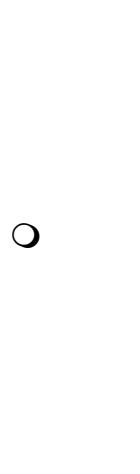 & 0 & 0 \\
\hline
\end{tabular}


DQ5 How likely are you to get 7 to 8 continuous hours of sleep each day because you feel...(with one being not at all likely and 5 being extremely likely)

\begin{tabular}{|c|c|c|c|c|c|}
\hline & $\begin{array}{r}\text { Not } \\
\text { at all likely (1) }\end{array}$ & (2) & (3) & (4) & $\begin{array}{l}\text { Extremely } \\
\text { likely (5) }\end{array}$ \\
\hline $\begin{array}{c}\text { Up-to- } \\
\text { date with your } \\
\text { work } \\
\text { responsibilities. } \\
\text { (DQ5_1) }\end{array}$ & 0 & $\mathrm{O}$ & 0 & 0 & 0 \\
\hline $\begin{array}{l}\text { Up-to- } \\
\text { date with your } \\
\text { personal } \\
\text { responsibilities } \\
\text { (for example, } \\
\text { chores). (DQ5_2) }\end{array}$ & 0 & 0 & 0 & 0 & 0 \\
\hline $\begin{array}{l}\text { Confident } \\
\text { in your finances. } \\
\text { (DQ5_3) }\end{array}$ & 0 & 0 & 0 & 0 & 0 \\
\hline $\begin{array}{l}\text { Confident } \\
\text { in the success of } \\
\text { your future job- } \\
\text { related goals (for } \\
\text { example, receiving } \\
\text { a raise or } \\
\text { promotion). } \\
\text { (DQ5_4) }\end{array}$ & 0 & 0 & 0 & 0 & 0 \\
\hline $\begin{array}{l}\text { Physically } \\
\text { tired (DQ5_5) }\end{array}$ & 0 & O & 0 & 0 & 0 \\
\hline $\begin{array}{c}\text { Mentally } \\
\text { tired (DQ5_6) }\end{array}$ & 0 & 0 & 0 & 0 & 0 \\
\hline
\end{tabular}


DQ6 In order to help you sleep 7 to 8 continuous hours each day, how confident are you that you can... (with one being not at all confident and 5 being extremely confident)

\begin{tabular}{|c|c|c|c|c|c|}
\hline & $\begin{array}{l}\text { Not at } \\
\text { all confident } \\
\text { (1) }\end{array}$ & (2) & (3) & (4) & $\begin{array}{l}\text { Extremely } \\
\text { confident (5) }\end{array}$ \\
\hline $\begin{array}{l}\text { Create a } \\
\text { bedroom } \\
\text { environment } \\
\text { suitable for } \\
\text { receiving good } \\
\text { sleep. (DQ6_1) }\end{array}$ & 0 & 0 & O & O & 0 \\
\hline $\begin{array}{l}\text { Relax } \\
\text { your mind before } \\
\text { bedtime. } \\
\text { (DQ6_2) }\end{array}$ & O & 0 & 0 & 0 & 0 \\
\hline $\begin{array}{l}\text { Relax } \\
\text { your body before } \\
\text { bedtime. } \\
\text { (DQ6_3) }\end{array}$ & 0 & 0 & O & O & 0 \\
\hline $\begin{array}{l}\text { Maintain } \\
\text { a consistent sleep } \\
\text { schedule. (for } \\
\text { example, going to } \\
\text { bed and waking } \\
\text { up at } \\
\text { approximately } \\
\text { the same time } \\
\text { each day). } \\
\text { (DQ6_4) }\end{array}$ & O & O & O & 0 & 0 \\
\hline $\begin{array}{c}\text { Not } \\
\text { consume } \\
\text { caffeine, alcohol, } \\
\text { or tobacco within } \\
4 \text { hours of } \\
\text { bedtime. } \\
\text { (DQ6_5) }\end{array}$ & 0 & O & 0 & O & $\mathrm{O}$ \\
\hline $\begin{array}{l}\text { Develop } \\
\text { goals to improve } \\
\text { your sleep habits. } \\
\text { (DQ6_6) }\end{array}$ & 0 & 0 & 0 & O & 0 \\
\hline
\end{tabular}


DQ7 How often do you... (with one being never and 5 being always)

\begin{tabular}{|c|c|c|c|c|c|}
\hline & (1) & (2) & (3) & (4) & $\begin{array}{l}\text { Always } \\
\text { (5) }\end{array}$ \\
\hline $\begin{array}{r}\text { Sleep } 7 \\
\text { to } 8 \text { continuous } \\
\text { hours each day. } \\
\text { (DQ7_1) }\end{array}$ & 0 & 0 & 0 & 0 & 0 \\
\hline $\begin{array}{l}\text { Sleep } \\
\text { less than } 7 \\
\text { continuous } \\
\text { hours each day. } \\
\text { (DQ7_2) }\end{array}$ & 0 & 0 & O & $\mathrm{O}$ & 0 \\
\hline $\begin{array}{l}\text { Sleep } \\
\text { more than } 8 \\
\text { continuous } \\
\text { hours each day. } \\
\text { (DQ7_3) }\end{array}$ & 0 & 0 & 0 & $O$ & 0 \\
\hline
\end{tabular}

DQ8 The following questions make use of "yes" and "no" responses.

\begin{tabular}{|c|c|c|}
\hline $\begin{array}{c}\text { Is getting enough sleep as } \\
\text { important to your overall health as } \\
\text { eating right and exercising } \\
\text { regularly? (DQ8_1) }\end{array}$ & No (2) \\
$\begin{array}{c}\text { Do you consume at least } 5 \\
\text { servings of fruits and vegetables } \\
\text { per day? (DQ8_2) } \\
\text { Have you ever }\end{array}$ & 0 & 0 \\
$\begin{array}{c}\text { unintentionally fallen asleep while } \\
\text { operating a motor vehicle? } \\
\text { (DQ8_3) }\end{array}$ & 0 & \\
\hline
\end{tabular}


Next.. we have few questions about your knowledge regarding sleep, drug use, medical disorders, and your weight and exercise habits.

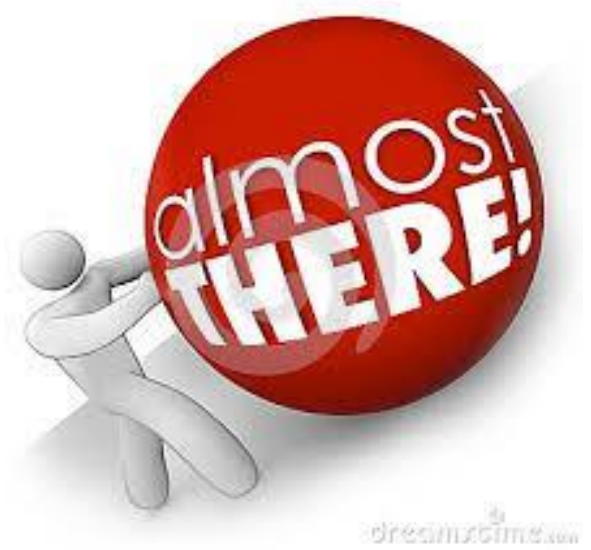


EQ1 The following questions assess your sleep health knowledge (The questions in this section make use of rating scale with five (5) answers, ranging from strongly agree to strongly disagree) Please indicate to what extent you agree or disagree with each statement 


\begin{tabular}{|c|c|c|c|c|c|}
\hline & $\begin{array}{l}\text { Strongly } \\
\text { agree (5) }\end{array}$ & (4) & $\begin{array}{l}\text { Neither } \\
\text { agree nor } \\
\text { disagree (3) }\end{array}$ & $\begin{array}{l}\text { Disagree } \\
\text { (2) }\end{array}$ & $\begin{array}{l}\text { Strongly } \\
\text { disagree (1) }\end{array}$ \\
\hline $\begin{array}{l}\text { People } \\
\text { need } 8 \text { hours of } \\
\text { sleep to feel } \\
\text { refreshed and } \\
\text { function well } \\
\text { during the day. } \\
\text { (EQ1_1) }\end{array}$ & 0 & 0 & 0 & 0 & 0 \\
\hline $\begin{array}{l}\text { When } \\
\text { people feel } \\
\text { tired, have no } \\
\text { energy, or just } \\
\text { seem not to } \\
\text { function well } \\
\text { during the day, it } \\
\text { is generally } \\
\text { because they did } \\
\text { not sleep well } \\
\text { the night before. } \\
\text { (EQ1_2) }\end{array}$ & 0 & 0 & 0 & 0 & 0 \\
\hline $\begin{array}{l}\text { A poor } \\
\text { night's sleep will } \\
\text { interfere with } \\
\text { activities the } \\
\text { next day. } \\
\text { (EQ1_3) }\end{array}$ & 0 & 0 & 0 & 0 & 0 \\
\hline $\begin{array}{l}\text { When } \\
\text { people feel } \\
\text { irritable, } \\
\text { depressed, or } \\
\text { anxious during } \\
\text { the day, it is } \\
\text { mostly because } \\
\text { they did not } \\
\text { sleep well the } \\
\text { night before. } \\
\text { (EQ1_4) }\end{array}$ & O & 0 & 0 & 0 & O \\
\hline $\begin{array}{l}\text { A good } \\
\text { night's sleep } \\
\text { means sleeping } \\
\text { like a log all } \\
\text { night. (EQ1_5) }\end{array}$ & 0 & 0 & 0 & 0 & O \\
\hline $\begin{array}{l}\text { Healthy } \\
\text { sleep means } \\
\text { falling asleep } \\
\text { quickly. (EQ1_6) }\end{array}$ & 0 & 0 & 0 & O & O \\
\hline
\end{tabular}




\begin{tabular}{|c|c|c|c|c|}
\hline $\begin{array}{c}\text { Faulty } \\
\text { practices around } \\
\text { sleep-wake } \\
\text { behaviors can } \\
\text { cause ongoing } \\
\text { sleep problems. } \\
\text { (EQ1_7) } \\
\text { Without }\end{array}$ & 0 & 0 & 0 & 0 \\
$\begin{array}{c}\text { an adequate } \\
\text { night's sleep it is } \\
\text { hard to function } \\
\text { the next day. } \\
\text { (EQ1_8) }\end{array}$ & 0 & 0 & 0 & 0 \\
\hline
\end{tabular}

The following questions assess the use of different drugs within the last 30 days

FQ1 This question ask about the Non-Medical use of Antidepressants, Painkillers, Sedatives, and Stimulants in the last 30 days: Within the last thirty days, have you taken any of the following prescription drugs that were not prescribed to you?

\begin{tabular}{|c|c|c|}
\hline & Yes (1) & No (2) \\
\hline $\begin{array}{l}\text { Antidepressants ( e.g., } \\
\text { Celexa, Lexapro, , Prozac, } \\
\text { Wellbutrin, Zoloft) (FQ1_1) }\end{array}$ & 0 & 0 \\
\hline $\begin{array}{l}\text { Pain killers ( e.g., } \\
\text { OxyContin, Vicodin, Codeine) } \\
\text { (FQ1_2) }\end{array}$ & 0 & 0 \\
\hline $\begin{array}{l}\text { Sedatives ( e.g., Xanaz, } \\
\text { Valium) (FQ1_3) }\end{array}$ & 0 & 0 \\
\hline $\begin{array}{l}\text { Stimulants ( e.g., Ritalin, } \\
\text { Adderall) (FQ1_4) }\end{array}$ & 0 & 0 \\
\hline
\end{tabular}


FQ2 The following questions ask about Other drugs/substances use including Marijuana and Cigarette. The question make use of a scales with eight (8) response options range from Never used to use Daily. Please indicate where you fall on the scale

Within the last thirty days, on how many days did you use:

\begin{tabular}{|c|c|c|c|c|c|c|c|c|}
\hline & $\begin{array}{l}\mathrm{N} \\
\text { ever used } \\
(8)\end{array}$ & $\begin{array}{l}H \\
\text { ave used, } \\
\text { but not in } \\
\text { last } 30 \text { (7) }\end{array}$ & $\begin{array}{c}-2 \text { days } \\
(6)\end{array}$ & $\begin{array}{c}-5 \text { days } \\
\text { (5) }\end{array}$ & $\begin{array}{c}-9 \text { days } \\
\text { (4) }\end{array}$ & $\begin{array}{c}0-19 \\
\text { days (3) }\end{array}$ & $\begin{array}{c}0-29 \\
\text { days (2) }\end{array}$ & $\begin{array}{l}\text { Ued daily } \\
\text { (1) }\end{array}$ \\
\hline $\begin{array}{r}\text { Marij } \\
\text { uana (FQ2__) }\end{array}$ & 0 & 0 & 0 & 0 & 0 & 0 & 0 & 0 \\
\hline $\begin{array}{r}\text { Cigar } \\
\text { ettes (FQ2_2) }\end{array}$ & 0 & 0 & 0 & 0 & 0 & 0 & 0 & 0 \\
\hline
\end{tabular}

The following questions are related to alcohol drinking habits, and Caffeinated and decaffeinate drinking habits. "A standard drink is defined as a 12 ounce bottle of beer, a 5 ounce glass of wine, or a 1.5 ounce shot of 80 proof liquor."

FQ3-1 Alcohol: In a TYPICAL WEEK, on how many days did you have a least one drink containing alcohol? Answers can range from zero (0) (you don't drink) to 7 days a week

FQ3-2 How many drinks do you have on a TYPICAL DAY when you are drinking? Put zero if you do not drink.

FQ3-3 During the last 30 days, what is the HIGHEST number of drinks that you consumed on any one occasion? Put zero if you do not drink.

FQ3-4 Please indicate how many drinks you have on average for each day of the week? Put zero if you do not drink.

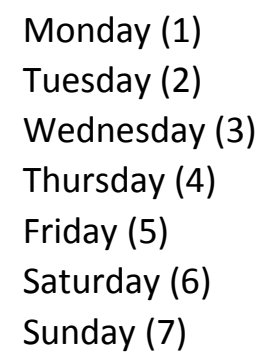


FQ4 During the last 7 days, how much in the equivalent of $12 \mathrm{oz}$ cans/glasses, did you drink of the following drinks every day?

FQ4-1 Coffee (not de-caffeinated)

O I did not (7)

One can/glass (6)

O Two cans/glasses (5)

O Three cans/glasses (4)

Four cans/glasses (3)

O Five cans/glasses (2)

Six cans/glasses or more (1)

FQ4-2 De-caffeinated coffee

O I did not (7)

One can/glass (6)

O Two cans/glasses (5)

O Three cans/glasses (4)

Four cans/glasses (3)

Five cans/glasses (2)

Six cans/glasses or more (1)

FQ4-3 Tea

O I did not (7)

One can/glass (6)

Two cans/glasses (5)

Three cans/glasses (4)

Four cans/glasses (3)

O Five cans/glasses (2)

O Six cans/glasses or more (1) 
FQ4-4 Soda/Pop that contains caffeine (Coca Cola, Pepsi Cola, Dr. Pepper, Mountain Dew, etc).

I did not (7)

One can/glass (6)

Two cans/glasses (5)

O Three cans/glasses (4)

Four cans/glasses (3)

Five cans/glasses (2)

Six cans/glasses or more (1)

FQ4-5 Energy drinks that contain caffeine (Red Bull, Monster, Rockstar, etc.).

O I did not (7)

One can/glass (6)

Two cans/glasses (5)

O Three cans/glasses (4)

Four cans/glasses (3)

Five cans/glasses (2)

Six cans/glasses or more (1)

You are almost done...Few more questions 
$G$ This section is interested in knowing whether you have one or more of the following disorders: Depression, Attention Deficit and Hyperactivity Disorder (ADHD), Insomnia, Sleep Disorders, and Stress.

GQ1 Have you ever been diagnosed with Depression?

$\begin{array}{ll}O & \text { Yes (1) } \\ \text { O } & \text { No (2) }\end{array}$

GQ2 The following questions make use of a scale with 6 response options, please indicate where you fall on the scale Within the last 12 months, have you been diagnosed or treated by a professional for any of the following:

\begin{tabular}{|c|c|c|c|c|c|c|}
\hline & $O(6)^{N}$ & $\begin{array}{r}\text { Yes } \\
\text {, diagnosed } \\
\text { not treated } \\
\text { (5) }\end{array}$ & $\begin{array}{l}\text { Yes } \\
\text {, treated } \\
\text { with } \\
\text { medication } \\
\text { (4) }\end{array}$ & $\begin{array}{c}\text { Yes, } \\
\text { treated with } \\
\text { psychotherap } \\
\text { y (3) }\end{array}$ & $\begin{array}{l}\text { Yes, } \\
\text { treated with } \\
\text { meds+ } \\
\text { psychotherap } \\
\text { y (2) }\end{array}$ & $\begin{array}{l}\text { Yes } \\
\text {, other } \\
\text { treatment } \\
\text { (1) }\end{array}$ \\
\hline $\begin{array}{l}\text { Attentio } \\
\text { n Deficit and } \\
\text { Hyperactivity } \\
\text { Disorder (ADHD) } \\
\text { (GQ2_1) }\end{array}$ & 0 & 0 & 0 & 0 & 0 & 0 \\
\hline $\begin{array}{l}\text { Insomni } \\
\text { a (GQ2_2) }\end{array}$ & 0 & 0 & 0 & 0 & 0 & 0 \\
\hline $\begin{array}{c}\text { Sleep } \\
\text { Disorder (GQ2_3) }\end{array}$ & 0 & 0 & 0 & 0 & 0 & 0 \\
\hline
\end{tabular}

GQ3 Within the last 12 months, how would you rate the overall level of stress you have experienced?

No stress (5)

Less than average stress (4)

Average stress (3)

O More than average stress (2)

O Tremendous stress (1)

Here we would like to know your height, weight, and exercises; and doing exercises, diet or using pills to lose weight. 
HQ1 Please indicate your height in feet and inches. What is your height in feet and inches?

Feet (1)

Inches (2)

HQ2 Please indicate your weight in pounds. What is your weight in pounds?

Pounds (1)

HQ3 On how many of the past 7 days did you

\begin{tabular}{|c|c|c|c|c|c|c|c|c|}
\hline & days (1) & day (2) & days (3) ${ }^{2}$ & days (4) & days (5) & days (6) & days (7) & days (8) \\
\hline $\begin{array}{c}\quad D \\
\text { o } \\
\text { moderate } \\
\text { intensity } \\
\text { cardio or } \\
\text { aerobic } \\
\text { exercises } \\
\text { for at least } \\
30 \\
\text { minutes? } \\
\text { (HQ3_1) }\end{array}$ & 0 & 0 & 0 & 0 & 0 & 0 & 0 & 0 \\
\hline $\begin{array}{c}\quad D \\
\text { o } \\
\text { moderate } \\
\text { vigorous } \\
\text { cardio or } \\
\text { aerobic } \\
\text { exercises } \\
\text { for at least } \\
20 \\
\text { minutes? } \\
\text { (HQ3_2) }\end{array}$ & 0 & 0 & 0 & 0 & 0 & 0 & 0 & 0 \\
\hline $\begin{array}{l}\text { D } \\
\text { o 8-12 } \\
\text { strength } \\
\text { training } \\
\text { exercises } \\
\text { for 8-12 } \\
\text { minutes } \\
\text { repetitions } \\
\text { each? } \\
\text { (HQ3_3) }\end{array}$ & 0 & 0 & 0 & 0 & 0 & 0 & 0 & 0 \\
\hline
\end{tabular}


HQ4 Within the last 30 days, did you do any of the following:

\begin{tabular}{|c|c|c|}
\hline & Yes (1) & No (2) \\
\hline $\begin{array}{c}\text { Exercises to lose weight? } \\
\text { (HQ4_1) }\end{array}$ & O \\
$\begin{array}{l}\text { Diet to lose weight? } \\
\text { (HQ4_2) }\end{array}$ & 0 & O \\
$\begin{array}{l}\text { Take pills lose weight? } \\
\text { (HQ4_3) }\end{array}$ & O & O \\
\hline
\end{tabular}

Well done! Just few questions left about your life at West Virginia University 
The next set of questions ask you general questions about yourself and your life at West Virginia University. These questions are designed to allow you to provide your thoughts and opinions about the school climate here at West Virginia University. (All questions in this section make use of rating scales with five (5) answers, ranging from strongly agree to strongly disagree)

IQ1 The next group of questions asks about your relationships with your professors. Your Professor Relationships

\begin{tabular}{|c|c|c|c|c|c|}
\hline & $\begin{array}{l}\text { Strongly } \\
\text { agree (A) } \\
\text { (5) }\end{array}$ & $\begin{array}{l}\text { Agree } \\
\text { (B) (4) }\end{array}$ & $\begin{array}{l}\text { Neither } \\
\text { agree nor } \\
\text { disagree (C) (3) }\end{array}$ & $\begin{array}{l}\text { Disagree } \\
\text { (D) (2) }\end{array}$ & $\begin{array}{l}\text { Strongly } \\
\text { disagree }(E)(1)\end{array}$ \\
\hline $\begin{array}{l}\text { Professors } \\
\text { understand my } \\
\text { problems. } \\
\text { (IQ1_20) }\end{array}$ & 0 & 0 & 0 & 0 & 0 \\
\hline $\begin{array}{l}\text { Professors } \\
\text { and staff seem to } \\
\text { take a real interest } \\
\text { in my future. } \\
\text { (IQ1_21) }\end{array}$ & 0 & 0 & O & O & 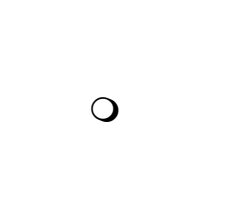 \\
\hline $\begin{array}{l}\text { Professors } \\
\text { are available when } \\
\text { I need to talk with } \\
\text { them. (IQ1_22) }\end{array}$ & 0 & $O$ & 0 & $O$ & $O$ \\
\hline $\begin{array}{l}\text { It is easy } \\
\text { to talk with } \\
\text { professors. } \\
\text { (IQ1_23) }\end{array}$ & 0 & 0 & 0 & 0 & $O$ \\
\hline $\begin{array}{l}\text { Students } \\
\text { get along well with } \\
\text { professors. } \\
\text { (IQ1_24) }\end{array}$ & 0 & 0 & 0 & 0 & $O$ \\
\hline $\begin{array}{l}\text { Professors } \\
\text { at my school help } \\
\text { us with our } \\
\text { problems. } \\
\text { (IQ1_25) }\end{array}$ & $\mathrm{O}$ & O & 0 & 0 & O \\
\hline $\begin{array}{c}\text { My } \\
\text { professors care } \\
\text { about me. } \\
\text { (IQ1_26) }\end{array}$ & 0 & 0 & 0 & 0 & 0 \\
\hline $\begin{array}{l}\text { My } \\
\text { professor makes } \\
\text { me feel good } \\
\text { about myself. } \\
\text { (IQ1_27) }\end{array}$ & $\mathrm{O}$ & 0 & 0 & 0 & 0 \\
\hline
\end{tabular}


IQ2 The next group of questions asks about your feelings of connectedness to West Virginia University (WVU). Your Connection to WVU

\begin{tabular}{|c|c|c|c|c|c|}
\hline & $\begin{array}{l}\text { Strongly } \\
\text { agree (A) (5) }\end{array}$ & $\begin{array}{l}\text { Agree } \\
\text { (B) }(4)\end{array}$ & $\begin{array}{c}\text { Neither } \\
\text { agree nor } \\
\text { disagree (C) (3) }\end{array}$ & $\begin{array}{l}\text { Disagree } \\
\text { (D) (2) }\end{array}$ & $\begin{array}{l}\text { Strongly } \\
\text { disagree (E) (1) }\end{array}$ \\
\hline $\begin{array}{c}\text { My } \\
\text { schoolwork is } \\
\text { exciting. (IQ2_28) }\end{array}$ & 0 & 0 & 0 & 0 & 0 \\
\hline $\begin{array}{l}\text { Students } \\
\text { can make } \\
\text { suggestions on } \\
\text { courses that are } \\
\text { offered. (IQ2_29) }\end{array}$ & 0 & 0 & 0 & 0 & 0 \\
\hline $\begin{array}{l}\text { WVU } \\
\text { makes students } \\
\text { enthusiastic } \\
\text { about learning. } \\
\text { (IQ2_30) }\end{array}$ & 0 & 0 & 0 & 0 & 0 \\
\hline $\begin{array}{l}\text { Students } \\
\text { are frequently } \\
\text { rewarded or } \\
\text { praised by faculty } \\
\text { and staff for } \\
\text { following } \\
\text { university rules. } \\
\text { (IQ2_31) }\end{array}$ & 0 & 0 & 0 & 0 & 0 \\
\hline
\end{tabular}


IQ3 The next group of questions asks about your feelings of academic support at

WVU. Your Academic Support

\begin{tabular}{|c|c|c|c|c|c|}
\hline & $\begin{array}{l}\text { Strongly } \\
\text { agree (A) } \\
\text { (5) }\end{array}$ & $\begin{array}{l}\text { Agree } \\
\text { (B) (4) }\end{array}$ & $\begin{array}{l}\text { Neither } \\
\text { agree nor } \\
\text { disagree (C) (3) }\end{array}$ & $\begin{array}{l}\text { Disagree } \\
\text { (D) (2) }\end{array}$ & $\begin{array}{r}\text { Strongly } \\
\text { disagree (E) (1) }\end{array}$ \\
\hline $\begin{array}{l}\text { I usually } \\
\text { understand my } \\
\text { homework } \\
\text { assignments. } \\
\text { (IQ3_32) }\end{array}$ & 0 & 0 & O & 0 & 0 \\
\hline $\begin{array}{l}\text { Professors } \\
\text { make it clear what } \\
\text { work needs to be } \\
\text { done to get the } \\
\text { grade I want. } \\
\text { (IQ3_33) }\end{array}$ & 0 & 0 & 0 & 0 & 0 \\
\hline $\begin{array}{l}\text { I believe } \\
\text { that professors } \\
\text { expect all students } \\
\text { to learn. (IQ3_34) }\end{array}$ & 0 & 0 & 0 & 0 & 0 \\
\hline $\begin{array}{l}\text { I feel that } \\
\text { I can do well at } \\
\text { WVU. (IQ3_35) }\end{array}$ & 0 & 0 & 0 & 0 & 0 \\
\hline
\end{tabular}


IQ4 The next group of questions asks about your feelings of order, safety, and discipline at WVU. WVU Rules, Safety, and Discipline

\begin{tabular}{|c|c|c|c|c|c|}
\hline & $\begin{array}{l}\text { Strongly } \\
\text { agree (A) } \\
\text { (5) }\end{array}$ & $\begin{array}{l}\text { Agree } \\
\text { (B) (4) }\end{array}$ & $\begin{array}{l}\text { Neither } \\
\text { agree nor } \\
\text { disagree (C) (3) }\end{array}$ & $\begin{array}{l}\text { Disagree } \\
\text { (D) (2) }\end{array}$ & $\begin{array}{l}\text { Strongly } \\
\text { disagree }(E)(1)\end{array}$ \\
\hline $\begin{array}{l}\text { Classroom } \\
\text { rules are applied } \\
\text { equally. (IQ4_36) }\end{array}$ & O & 0 & 0 & 0 & 0 \\
\hline $\begin{array}{l}\text { Problems } \\
\text { at WVU are solved } \\
\text { by students and } \\
\text { faculty. (IQ4_37) }\end{array}$ & 0 & 0 & $\mathrm{O}$ & 0 & 0 \\
\hline $\begin{array}{l}\text { The rules } \\
\text { of the university } \\
\text { are fair. (IQ4_38) }\end{array}$ & 0 & 0 & 0 & 0 & 0 \\
\hline $\begin{array}{l}\text { WVU } \\
\text { rules are enforced } \\
\text { consistently and } \\
\text { fairly. (IQ4_39) }\end{array}$ & 0 & 0 & 0 & 0 & 0 \\
\hline $\begin{array}{l}\text { My } \\
\text { professors make it } \\
\text { clear to me when I } \\
\text { have misbehaved } \\
\text { in class. (IQ4_40) }\end{array}$ & 0 & 0 & 0 & $\mathrm{O}$ & 0 \\
\hline $\begin{array}{l}\text { Discipline } \\
\text { is fair. (IQ4_41) }\end{array}$ & 0 & 0 & 0 & $\mathrm{O}$ & O \\
\hline
\end{tabular}


IQ5 The next group of questions asks about your feelings of WVU's physical appearance. WVU's Physical Appearance

\begin{tabular}{|c|c|c|c|c|c|}
\hline & $\begin{array}{c}\text { Strongly } \\
\text { agree (A) (5) }\end{array}$ & $\begin{array}{l}\text { Agree } \\
\text { (B) (4) }\end{array}$ & $\begin{array}{c}\text { Neither } \\
\text { agree nor } \\
\text { disagree (C) (3) }\end{array}$ & $\begin{array}{l}\text { Disagree } \\
\text { (D) (2) }\end{array}$ & $\begin{array}{l}\text { Strongly } \\
\text { disagree }(E)(1)\end{array}$ \\
\hline $\begin{array}{l}\text { The } \\
\text { university } \\
\text { grounds are } \\
\text { kept clean. } \\
\text { (IQ5_42) }\end{array}$ & 0 & 0 & 0 & 0 & O \\
\hline $\begin{array}{l}\text { WVU is } \\
\text { neat and clean. } \\
\text { (IQ5_43) }\end{array}$ & 0 & 0 & 0 & 0 & 0 \\
\hline $\begin{array}{l}\text { WVU's } \\
\text { buildings are } \\
\text { generally } \\
\text { pleasant and } \\
\text { well } \\
\text { maintained. } \\
\text { (IQ5_44) }\end{array}$ & $\mathrm{O}$ & 0 & 0 & 0 & 0 \\
\hline $\begin{array}{l}\text { WVU is } \\
\text { usually clean } \\
\text { and tidy.. } \\
\text { (IQ5_45) }\end{array}$ & 0 & 0 & 0 & 0 & 0 \\
\hline
\end{tabular}

IQ6 The next group of questions asks about your feelings of WVU's social atmosphere. WVU's Social Atmosphere

\begin{tabular}{|c|c|c|c|c|c|}
\hline & $\begin{array}{l}\text { Strongly } \\
\text { agree (A) (5) }\end{array}$ & $\begin{array}{l}\text { Agree } \\
\text { (B) (4) }\end{array}$ & $\begin{array}{c}\text { Neither } \\
\text { agree nor } \\
\text { disagree (C) (3) }\end{array}$ & $\begin{array}{l}\text { Disagree } \\
\text { (D) (2) }\end{array}$ & $\begin{array}{l}\text { Strongly } \\
\text { disagree (E) (1) }\end{array}$ \\
\hline $\begin{array}{l}\text { I am } \\
\text { happy with } \\
\text { kinds of } \\
\text { students who } \\
\text { go to WVU. } \\
\text { (IQ6_46) }\end{array}$ & 0 & 0 & 0 & 0 & 0 \\
\hline $\begin{array}{l}\qquad \text { I am } \\
\text { happy, in } \\
\text { general, with } \\
\text { the other } \\
\text { students who } \\
\text { go to WVU. } \\
\text { (IQ6_47) }\end{array}$ & 0 & 0 & 0 & 0 & 0 \\
\hline
\end{tabular}


IQ7 The next group of questions asks about your feelings of your ability to participate in WVU activities. Your WVU Participation 


\begin{tabular}{|c|c|c|c|c|c|}
\hline & $\begin{array}{c}\text { Strongly } \\
\text { agree (A) (5) }\end{array}$ & $\begin{array}{l}\text { Agree } \\
\text { (B) }(4)\end{array}$ & $\begin{array}{l}\text { Neither } \\
\text { agree nor } \\
\text { disagree (C) (3) }\end{array}$ & $\begin{array}{l}\text { Disagree } \\
\text { (D) (2) }\end{array}$ & $\begin{array}{l}\text { Strongly } \\
\text { disagree }(E)(1)\end{array}$ \\
\hline $\begin{array}{l}\text { At WVU, } \\
\text { the same person } \\
\text { always gets to } \\
\text { help the } \\
\text { professor. } \\
\text { (IQ7_49) }\end{array}$ & 0 & 0 & 0 & 0 & 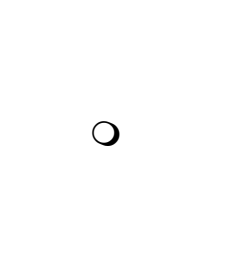 \\
\hline $\begin{array}{l}\text {. At } \\
\text { WVU, the same } \\
\text { students get } \\
\text { chosen every } \\
\text { time to take part } \\
\text { in after-school or } \\
\text { special activities. } \\
\text { (IQ7_50) }\end{array}$ & 0 & 0 & 0 & 0 & 0 \\
\hline $\begin{array}{l}\text { The } \\
\text { same students } \\
\text { always get to use } \\
\text { things, like a } \\
\text { computer, or } \\
\text { other resources } \\
\text { when we interact } \\
\text { (IQ7_51) }\end{array}$ & 0 & 0 & 0 & $O$ & 0 \\
\hline $\begin{array}{l}\quad \text { Students } \\
\text { have the same } \\
\text { opportunity to } \\
\text { speak, and be } \\
\text { listened to, in } \\
\text { class. (IQ7_52) }\end{array}$ & 0 & 0 & 0 & 0 & 0 \\
\hline $\begin{array}{l}\text { Students } \\
\text { can express their } \\
\text { feelings and } \\
\text { thoughts about } \\
\text { school work and } \\
\text { life. (IQ7_53) }\end{array}$ & 0 & 0 & 0 & 0 & 0 \\
\hline $\begin{array}{l}\text { Students } \\
\text { who are } \\
\text { 'different' in any } \\
\text { way are treated } \\
\text { with respect. } \\
\text { (IQ7_54) }\end{array}$ & 0 & 0 & 0 & 0 & 0 \\
\hline $\begin{array}{l}\text { Nobody } \\
\text { at WVU is } \\
\text { excluded from } \\
\text { being successful. } \\
\text { (IQ7_55) }\end{array}$ & 0 & 0 & 0 & 0 & 0 \\
\hline
\end{tabular}




\begin{tabular}{|c|c|c|c|c|c|}
\hline $\begin{array}{l}\text { Females } \\
\text { and males are } \\
\text { treated as equals } \\
\text { at WVU. (IQ7_56) }\end{array}$ & 0 & 0 & 0 & 0 & 0 \\
\hline $\begin{array}{l}\text { I can } \\
\text { participate in a } \\
\text { lot of interesting } \\
\text { activities at } \\
\text { WVU. (IQ7_57) }\end{array}$ & 0 & 0 & 0 & O & 0 \\
\hline
\end{tabular}

IQ8 The next group of questions asks about your satisfaction with your schoolwork.

Your Academic Satisfaction

\begin{tabular}{|c|c|c|c|c|c|}
\hline & $\begin{array}{l}\text { Strongly agree } \\
\text { (A) (5) }\end{array}$ & $\begin{array}{l}\text { Agree } \\
\text { (B) (4) }\end{array}$ & $\begin{array}{c}\text { Neither } \\
\text { agree nor } \\
\text { disagree (C) (3) }\end{array}$ & $\begin{array}{l}\text { Disagree } \\
\text { (D) (2) }\end{array}$ & $\begin{array}{l}\text { Strongly } \\
\text { disagree }(E)(1)\end{array}$ \\
\hline $\begin{array}{l}\text { I am } \\
\text { happy about } \\
\text { the number of } \\
\text { tests I have. } \\
\text { (IQ8_58) }\end{array}$ & 0 & O & $\bigcirc$ & 0 & 0 \\
\hline $\begin{array}{l}. \text { I am } \\
\text { happy about } \\
\text { the amount of } \\
\text { homework I } \\
\text { have. (IQ8_59) }\end{array}$ & 0 & O & 0 & 0 & 0 \\
\hline $\begin{array}{l}\text { I feel } \\
\text { appropriately } \\
\text { academically } \\
\text { challenged at } \\
\text { WVU. (IQ8_60) }\end{array}$ & 0 & O & 0 & 0 & 0 \\
\hline
\end{tabular}


You are moving to the last section ( Demographic Information)...you will be done

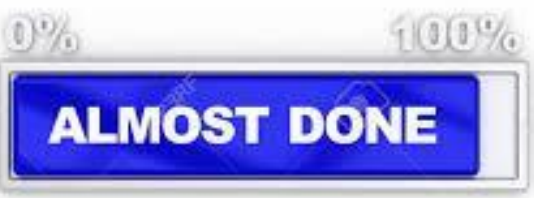


All responses in this section will be kept confidential and private. Only group data will be reported.

JQ1 What is your gender?

O Male (1)

Female (2)

JQ2 What is your age?

JQ3 How do you usually describe yourself?

O White (1)

Black or African American (2)

American Indian or Alaska Native (3)

Asian (4)

Native Hawaiian or Pacific Islander (5)

O Other (6)

JQ4 Do you have a part-time or full-time job?

Employed full time (1)

Employed part time (2)

O Unemployed (3)

JQ5 What is your annual income?

Less than $\$ 10,000$ (1)

O $\$ 10,000-\$ 19,999$ (2)

O $\$ 20,000-\$ 29,999$ (3)

O $\$ 30,000-\$ 39,999$ (4)

O More than $\$ 40,000$ (5)

JQ6 What is your marital status?

O Married (1)

Widowed (2)

O Divorced (3)

O Separated (4)

O Never married (5) 
JQ7 What is your year of enrollment?

O Freshman (1)

Sophomore (2)

junior (3)

Senior (4)

JQ8 What is your college major?

JQ9 What is your current overall Grade Point Average? (please write your response using a 4.0 scale)

JQ10 What time do your Friday classes start?

8 am (1)

Between 8 AM and $10 \mathrm{AM}(2)$

Between $10 \mathrm{AM}$ and noon (3)

Afternoon (4)

I do not have Friday classes (5)

You are done...Great Job! Thank you for your time and participation! NOW....Click next to be entered in a drawing for Panera gift card

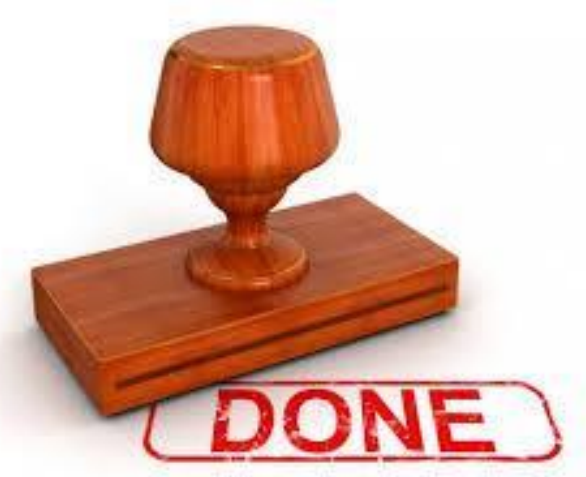


PC1 If you wish to be entered in a drawing to receive a Panera gift card...Click Yes Clicking NO will end the survey without...

Yes (28)

No (29) 
PC2 Please enter your e-mail address, so that you can be included in a drawing for a Panera giftcard. This information will not be associated with your data.

E-mail address (1) 


\section{Appendix E}

\section{Corresponding letters}




\section{WestVurginiaUniversity.}

SCHOOL OF PUBLIC HEALTH

\section{PRENOTIFICATION E-MAIL MESSAGE}

\section{Dear student,}

We need your help! You have been randomly selected to participate in an important universitywide web-based Sleep Behavior Questionnaire of West Virginia undergraduate students. It has been developed so you, the student body here at West Virginia University, can tell us what you think, believe and do that may affect your sleep. This study is being conducted by Dr. Keith Zullig and PhD student Yahya Alamir at West Virginia University. This study has been reviewed and approved by the Institutional Review Boards (IRB) at West Virginia University (WVU) IRB's Research Protocol: (WVU IRB's Research Protocol: 1603066826).

This research is part of a larger study, and it is being conducted in partial fulfillment of the requirements for the degree of Doctor of Philosophy in Public Health / Social and Behavioral Sciences. The project is intended to describe college students' sleep and their behavioral determinants in order to better understand sleep behavior of undergraduate college students. Additionally, we are interested in assessing the association between sleep behavior, particularly poor sleep and health related quality of life. Your involvement in this study is voluntary and your responses will be kept confidential. In addition, you do not have to answer every question in the survey. Therefore, if you are not comfortable with a particular question, simply leave it blank and go on to the next. No action will be taken against you; your class standing and grades will not be affected whether you complete this questionnaire, choose not to participate, or by withdrawal from the study.

In order to participate in this study, you must be:

- 18 years old or older

- A full time, undergraduate student at West Virginia University.

The information you provide will assist our university health community to develop better health education/wellness efforts at the University. Your participation is very important to us.

Doing this web-based questionnaire will cause no risk to you and your results will be held strictly confidential. As a token of our appreciation for your participation, you will have a chance to win one of 50 Panera gift cards ( $\$ 10$ value each). In five days, you will receive an e-mail requesting your participation. We hope you will take the time to participate. Please call or e-mail us with questions or concerns. You may also contact the Office of Research Integrity and Compliance (293-7073, ORIC@mail.wvu.edu) for any questions about your rights as a participant in this study.

\section{Sincerely,}

Keith J. Zullig, MSPH, PhD Tel: (304) 293-1091; E-mail: kzullig@hsc.wvu.edu

Yahya A. Alamir, MAP, PhD Candidate Tel: (681) 753-8223; E-mail: yalamir@mix.wvu.edu 


\section{WestVurginiaUniversity。}

SCHOOL OF PUBLIC HEALTH

\section{REQUESTING SURVEY PARTICIPATION}

\section{Dear Student,}

Five days ago, you were sent an e-mail requesting your participation in the University-wide Web-based Sleep Behavior Questionnaire of West Virginia Undergraduate Students. Our hopes are that you are excited about being selected to participate in this opportunity to represent the West Virginia student body. We are certainly excited to be able to gather information that will continue to help make your experience at West Virginia University an enjoyable one and to make the University a better place for future classes.

Please click on the web link below to get to the informed consent form and questionnaire (or you may copy the link and past the link into your internet browser).

It is very important that you read and understand what the informed consent form states. We take your responses very seriously and have taken every precaution to assure the confidentiality of your results. But most all, enjoy the experience, and please call or e-mail us with any questions or concerns. You may also contact the Office of Research Integrity and Compliance (293-7073, ORIC@ mail.wvu.edu) for any questions about your rights as a participant in this study.

$$
\text { http://wvu.qualtrics.com/SE/? SID=S V_831B1Oe yAYK4bgF }
$$

Sincerely

Keith J. Zullig, MSPH, PhD Tel: (304) 293-1091; E-mail: kzullig@hsc.wvu.edu Yahya A. Alamir, MAP, PhD Student Tel: (681) 753-8223; E-mail: yalamir@mix.wvu.edu 


\section{WestVriginiaUniversity。}

SCHOOL OF PUBLIC HEALTH

"Please ignore this letter if you have already completed the survey"

\section{Dear Student,}

One week ago, you were sent an e-mail asking for your participation in a Web-based universitywide sleep behavior questionnaire. If you have not yet had a chance to complete the questionnaire, won't you please take a few minutes now to fill it out. Simply click on the link below to get started!

Because you are one of a small selected group to participate in this important study, your response is essential to assure valid results. Furthermore, you will have a chance to win one of 50 Panera gift cards (\$10 value each), for your time!

If you have already replied, thank you for your cooperation. Please contact Yahya Alamir or Keith Zullig if you have any additional questions about the study or the Office of Research Integrity and Compliance (293-7073, ORIC@ mail.wvu.edu) about your rights as a participant in this study.

$$
\text { http://wvu.qualtrics .com/SE/?SID=S V_831B1Oe yAYK4bgF }
$$

\section{Sincerely,}

Keith J. Zullig, MSPH, PhD Tel: (304) 293-1091; E-mail: kzullig@hsc.wvu.edu

Yahya A. Alamir, MAP, PhD Student Tel: (681) 753-8223; E-mail: yalamir@ mix.wvu.edu 


\section{WestVriginiaUniversity.}

SCHOOL OF PUBLIC HEALTH

SECOND E-MAIL REMINDER "Please ignore this letter if you have already completed the survey"

\section{Dear Student,}

If you have not yet had a chance to complete the West Virginia University sleep behavior questionnaire, this is your second chance to not only represent your student body, but also to better assist our university health community in developing better health education/wellness efforts at the University.

Won't you please take a few minutes now to fill it out? You can begin by going to the website below. Your response is essential to assure valid results.

Thank you again for your cooperation if you have already replied. Please contact Yahya Alamir or Keith Zullig if you have any additional questions about the study or the Office of Research Integrity and Compliance (293-7073, ORIC@mail.wvu.edu) about your rights as a participant in this study.

\section{http://wvu.qualtrics.com/SE/? S ID=S V 831B10eyAYK4bgF}

\section{Sincerely,}

Keith J. Zullig, MSPH, PhD Tel: (304) 293-1091; E-mail: kzullig@ hsc.wvu.edu

Yahya A. Alamir, MAP, PhD Student Tel: (681) 753-8223; E-mail: yalamir@ mix.wvu.edu 


\section{WestlirginiaUniversitys}

SCHOOL OF PUBLIC HEALTH

LAST REMINDER "Please ignore this letter if you have already completed the survey"

\section{Dear student ,}

If you have not yet had a chance to complete the West Virginia University sleep behavior questionnaire, this is your last chance to not only represent your student body, but also to better assist our university health community in developing better health education/wellness efforts at the University.

Won't you please take a few minutes now to fill it out? You can begin by going to the website below. Your response is essential to assure valid results.

Thank you again for your cooperation if you have already replied. Please contact Yahya Alamir or Keith Zullig if you have any additional questions about the study or the Office of Research Integrity and Compliance (293-7073, ORIC@mail.wvu.edu) about your rights as a participant in this study.

$$
\text { http://wvu.qualtrics.com/SE/? SID=SV_831B1OeyAYK4bgF }
$$

\section{Sincerely,}

Keith J. Zullig, MSPH, PhD Tel: (304) 293-1091; E-mail: kzullig@hsc.wvu.edu

Yahya A. Alamir, MAP, PhD Student Tel: (681) 753-8223; E-mail: yalamir@mix.wvu.edu 


\section{WVU Registrar letter aproval}

From: Thomas Snider [mailto:Tom.Snider@mail.wvu.edu]

Sent: Monday, May 16, 2016 10:38 AM

To: Zullig, Keith <kzullig@hsc.wvu.edu>

Subject: Re: WVU-Office of the University Registrar Internal Data Request

Good morning Keith,

Per our conversation, the Office of the University Registrar would be happy to provide you with a list of student MIX email addresses necessary to complete your research project after we receive the approval from the Institutional Review Board. The approval is necessary before we can disseminate any information that is to be used in research from our office.

If you have any questions, etc., please contact me directly.

Thomas L. Snider Assistant Registrar West Virginia University

P: (304)293-5355 F: (304)293-9891 
Appendix F

IRB Approval 


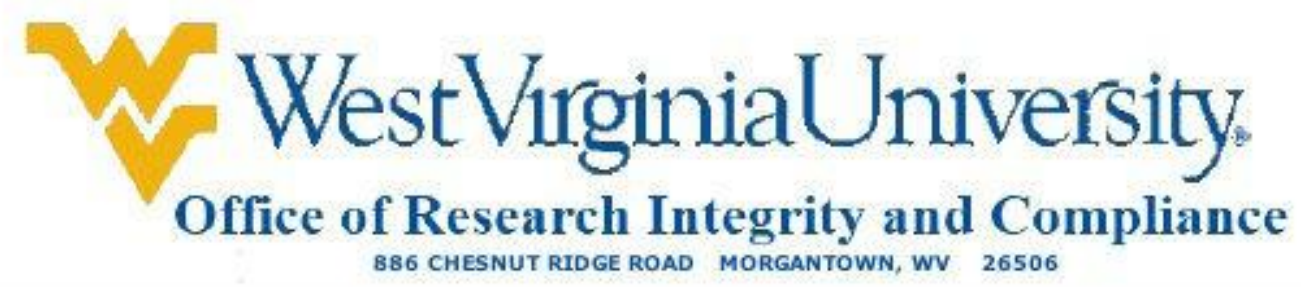

\section{Approval Letter Full Board}

Action Date 07/06/2016

To Keith Zullig

From WVU Office of Research Integrity and Compliance

Approval Date 05/25/2016

Expiration Date 05/24/2017

Subject Protocol Approval Letter

Protocol Number 1603066826

Title A Theory-based Description of Sleep Behavior and Its Impact on Health-Related

Quality Of Life Using the Health Belief Model and the Theory of Planned Behavior

The above-referenced research study was reviewed by the West Virginia University Institutional Review Board IRB via full board review procedures and was approved.

- At the full Board meeting of May 25, 2016 of the WVU IRB the Board reviewed the above-referenced study and voted to approve pending modifications. In response to the IRB's requested modifications, the researcher submitted revisions to the IRB. Based on the response, the IRB has approved the above-

referenced study for a period of twelve months. The review of this protocol was conducted and approved in accordance with the federally defined categories of expedited review outlined in 45 CFR

46.110(a) on July 6, 2016. The approval period is from May 25, 2016 through May 24, 2017.

Documents reviewed and/or approved as part of this submission:

E-MAIL MESSAGE REQUESTING SURVEY PARTICIPATION.pdf: 2016-07-01-04:00

Updated first Email reminder.pdf: 2016-05-30-04:00

SECOND EMAIL REMINDER (1).pdf: 2016-05-30-04:00

THIRD EMAIL REMINDER.pdf: 2016-05-30-04:00 
E-MAIL MESSAGE REQUESTING SURVEY PARTICIPATION.pdf: 2016-05-30-04:00 